\title{
LOS RAFIDIÓPTEROS DE LA PENÍNSULA IBÉRICA (INSECTA, NEUROPTERIDA: RAPHIDIOPTERA)
}

\author{
Víctor J. Monserrat ${ }^{*} \&$ David Papenberg ${ }^{* *}$ \\ Departamento de Zoología y Antropología Física. Facultad de Biología, Universidad Complutense, E-28040 Madrid, Spain \\ *E-mail: artmad@bio.ucm.es; **E-mail: david@gathisteria.com
}

\section{RESUMEN}

Se revisan las especies de rafidiópteros presentes en la fauna de la Península lbérica donde, hasta la fecha, están representados por 16 especies pertenecientes a 9 géneros de las dos familias actuales. Tras una diagnosis del orden y de su historial paleontológico, se aportan algunos datos generales sobre su morfología y biología, estadios juveniles, comportamiento y distribución, así como de la terminología utilizada, y se comenta una breve introducción sobre la historia en el conocimiento del orden, y en particular en la Península lbérica. Se incluye una lista de las especies pertenecientes a su fauna y una clave de identificación de las familias, géneros y especies citadas en la Península lbérica. De cada uno de estos taxa se recopilan y se anotan todas las referencias bibliográficas existentes, sean generales o relativas a las especies de la zona estudiada, tanto con la denominación actualmente aceptada, como de sus sinonimias y/o cualquier otra combinación nomenclatural bajo las cuales han sido citadas. También de cada especie se anotan los datos conocidos sobre su morfología externa, genital, variabilidad, estadios juveniles, biología, fenología y distribución altitudinal y geográfica en la península, aportando nuevo material mayoritariamente inédito (1.073 ejemplares estudiados) y los correspondientes mapas de su distribución conocida de estas especies en la zona estudiada.

Palabras clave: Insecta; Raphidioptera; Revisión; Taxonomía; Faunística; Morfología; Península lbérica; España; Portugal.

\section{ABSTRACT}

\section{The snake-flies from the Iberian Peninsula (Insecta, Neuropterida: Raphidioptera)}

The present contribution revises the snake-fly species present in the fauna of the lberian Peninsula where, to date, is represented by 16 species belonging to 9 genera of both actual families. After a diagnosis of the order and its paleontological history, there are exposed some general data on its morphology and biology, juvenile stages, behavior and distribution, as well as the used terminology, and is commented a brief introduction on the history in the knowledge of the order, and in particular in the Iberian Peninsula. It is included a list of the species belonging to the Iberian fauna and an identification key of families, genera, and species listed in the lberian Peninsula. For every one of these taxa are compiled and annotated all the existing bibliographic general references, as those relating to the species present in the studied area, both with the currently accepted name, their synonyms and/ or any other nomenclatural combinations under which they have been recorded. Of each species is also detailed the present knowledge of its external morphology, genital variability, juvenile stages, biology, phenology and altitudinal and geographic distribution in the Peninsula, providing the now studied material, mostly unrecorded (1,073 studied specimens), and the corresponding distribution maps of these species in the studied area.

Key words: Insecta; Raphidioptera; Revision; Taxonomy; Faunistics; Morphology; Iberian Peninsula; Spain; Portugal.

Recibido/Received: 16/07/2014; Aceptado/Accepted: 13/03/2015; Publicado en línea/Published online: 09/06/2015

Cómo citar este artículo/Citation: Monserrat, V. J. \& Papenberg, D. 2015. Los rafidiópteros de la Península lbérica (Insecta, Neuropterida: Raphidioptera). Grael/sia, 71(1): e024. http://dx.doi.org/10.3989/graellsia.2015.v71.116

Copyright: (C) 2015 SAM y CSIC. Salvo indicación contraria, todos los contenidos de la edición electrónica de Graellsia se distribuyen bajo licencia de uso y distribución Creative Commons Reconocimiento no Comercial 3.0. España (cc-by-nc). 


\section{Introducción}

GENERALIDADES: DIAGNOSIS, HISTORIAL PALEONTOLÓGICO Y DISTRIBUCIÓN

Los Raphidioptera Latreille, 1810, junto a los Megaloptera Latreille, 1810 y los Neuroptera s. str. (=Planipennia) Handlirsch, 1908 forman el Superorden Neuropterida o Neuroptera $s$. $l$, que está constituido por unas 6.520 especies repartidas en sus tres órdenes citados y habitualmente reconocidos: Megaloptera (c. 310 spp.), Raphidioptera (c. 239 spp.) y Planipennia o Neuroptera s. str. (c. 6.000 spp.) (H. Aspöck et al., 1980a, 1991, 2001), cuyas relaciones aún son objeto de amplios estudios y debates (ej.: Whiting et al., 1997; Ren \& Hong, 1994; Winterton et al., 2010; Haring et al., 2011; U. Aspöck et al., 2001, 2012; Peters et al., 2014).

El superorden está representado en la fauna del Paleártico Occidental (desde Macaronesia, Norte de África y Europa a Oriente Medio, Cáucaso e Irán) por c. 800 especies (H. Aspöck, 1992; H. Aspöck et al., 2001). La Fauna Ibérica posee representantes de todas las familias presentes en la Fauna neuropterológica Europea, y es particularmente rica en especies de neurópteros, ya que nada menos que 197 especies están presentes en su fauna: Megaloptera (3 spp.),

1

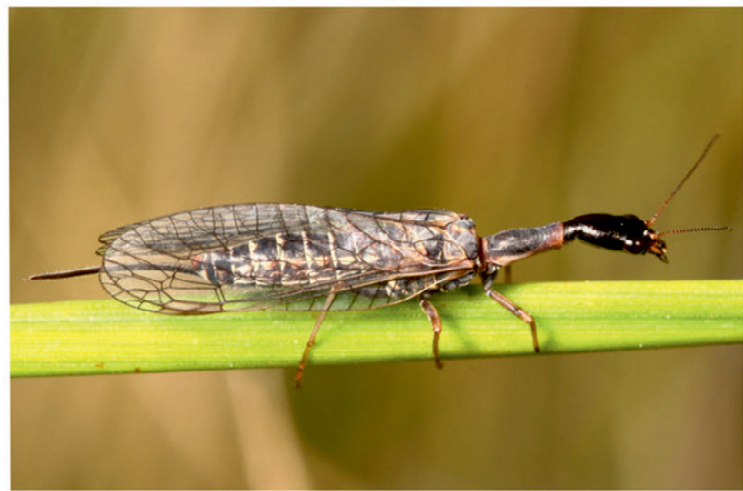

3

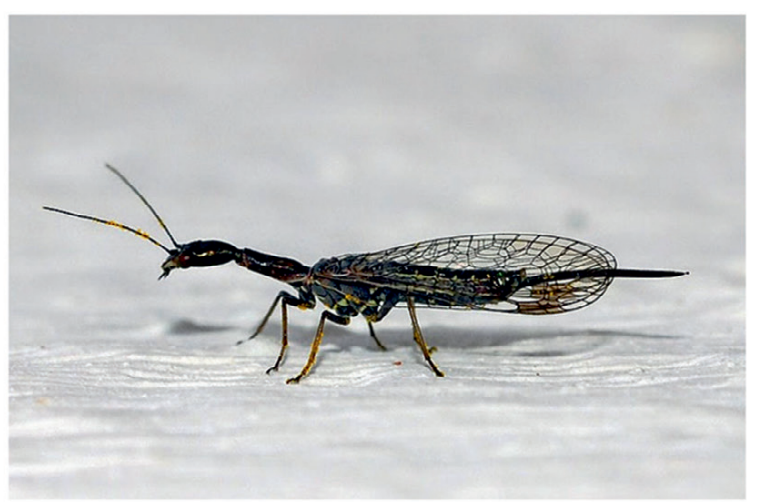

Raphidioptera (16 spp.) y Planipennia o Neuroptera s. str. (178 spp.), representando en varias familias entre el $100 \%$ a más del $75 \%$ del total de especies europeas conocidas (H. Aspöck et al., 1980a, 1991, 2001; H. Aspöck, 1992; H. Aspöck \& Hölzel, 1996; Monserrat \& Triviño, 2013).

El orden Raphidioptera posee dos familias actuales (Raphidiidae e Inocelliidae), y tiene como caracteres diagnósticos, entre otros, la presencia de cabeza prognata, aplanada y triangular, con dos grandes ojos compuestos y tres ocelos dorsales en Raphidiidae, y más cuadrada y sin ocelos presentes en Inocelliidae (figs. 5-8). Las antenas son filiformes, pudiendo ser, en alguna ocasión, moniliformes en la familia Inocelliidae (figs. 1-4) y el aparato bucal es masticador con palpo maxilar formado por 5 artejos y labial por 3. Destaca el característico protórax, muy alargado y móvil (figs. 1-4, 9-11). Las alas están bien desarrolladas, son subiguales, y presentan membrana hialina con pterostigma conspicuo (figs. 1-4, 24-39). La venación se caracteriza por la terminación de la vena Sc en el margen alar, por la ramificación de las venas longitudinales en el margen alar y por sus escasas venas transversales, que forman grandes celdillas (figs. 24-39). Las patas son marchadoras con el tercer tarsómero dilatado en forma de corazón (fig. 15),

2

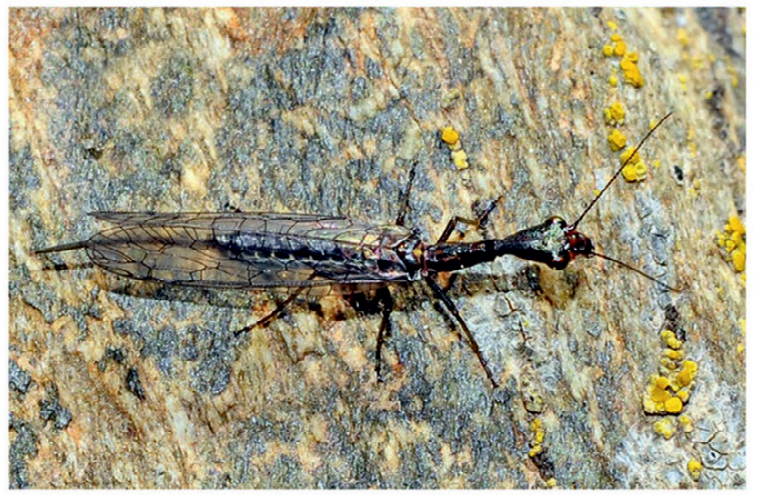

4

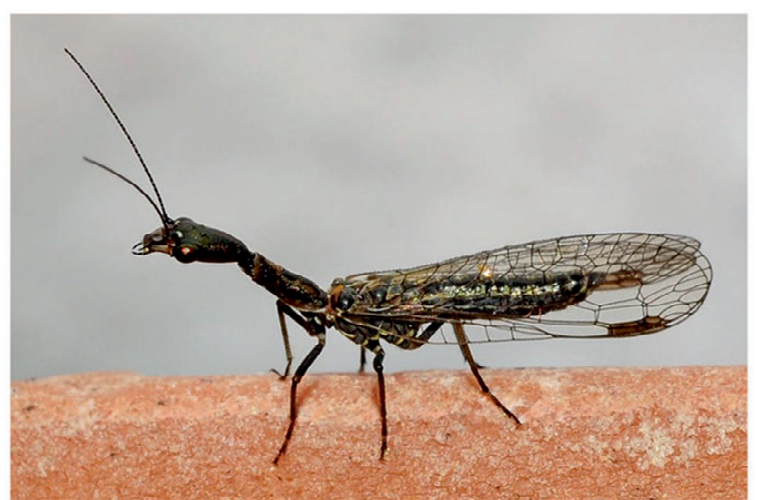

Figs.1-4.- Diferentes aspectos de rafidiópteros ibéricos en su hábitat natural. Foto 1 de Jorge Almeida, de http://www.flickr.com/ photos/superegnum/. Fotos 2-4 de Francisco Rodríguez, de http://faluke.blogspot.com.es/search/label/neuroptera.

Figs. 1-4.- Different aspects of lberian Raphidioptera in their natural habitats. Photo 1 of Jorge Almeida, from http://www.flickr. com/photos/superegnum/. Photos 2-4 of Francisco Rodríguez, from http://faluke.blogspot.com.es/search/label/neuroptera. 
5

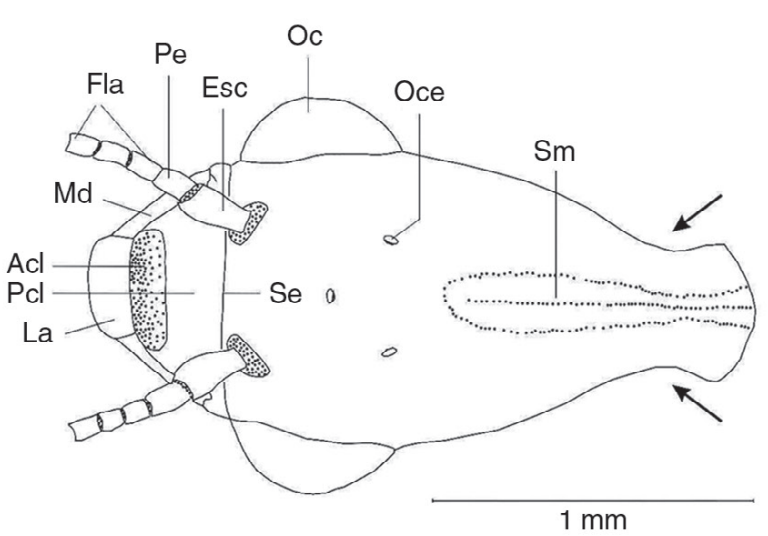

7
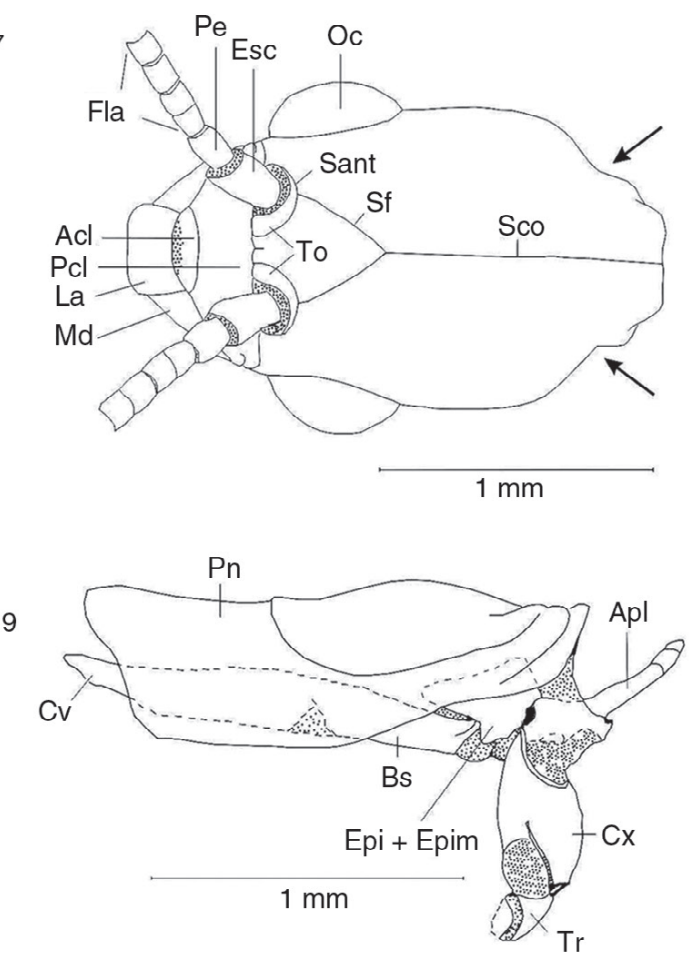

6
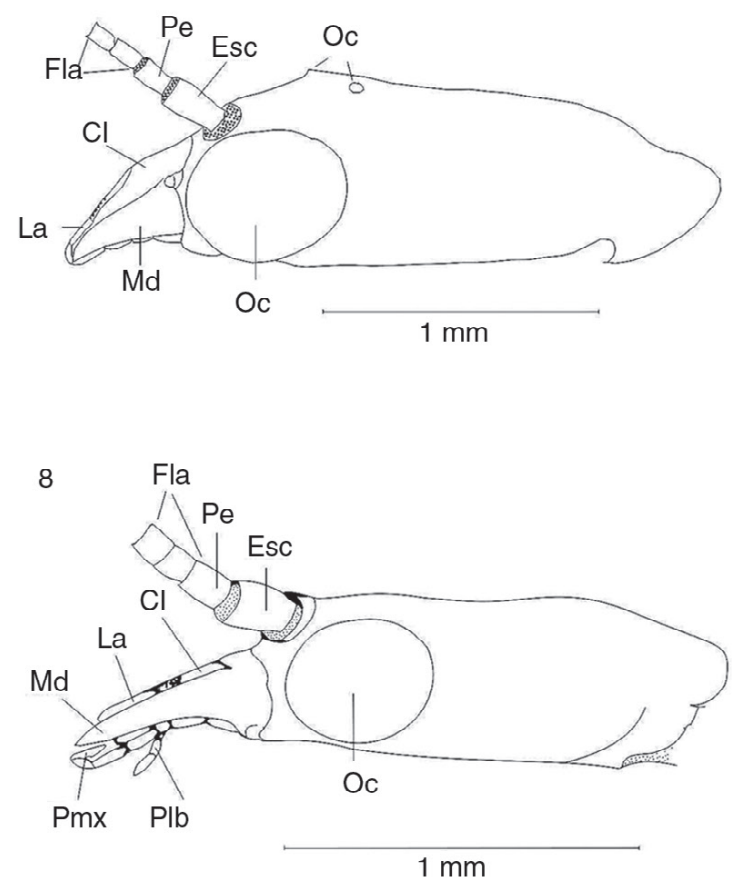

10

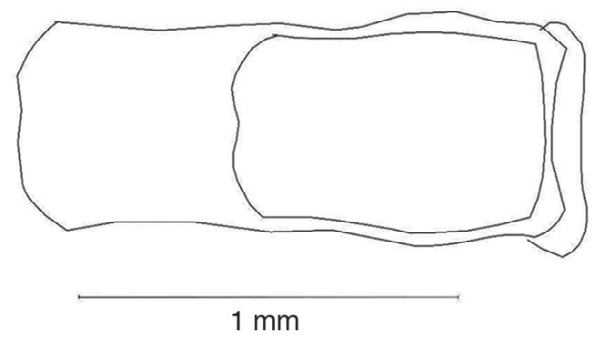

11

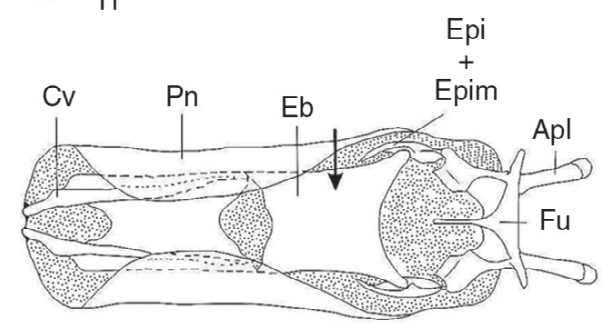

$1 \mathrm{~mm}$

Figs. 5-11. - Cabeza ( $\left({ }^{\prime}\right)$ en vista dorsal y lateral de Harraphidia (F.) laufferi (5-6) y de Parainocellia (P.) ressli (7-8). Pronoto (ठ) en vista lateral (9), dorsal (10) y ventral (11) de Harraphidia (F.) laufferi. Acl: anteclípeo, Apl: apófisis pleural, Cl: clípeo, Cv: cervicalium, Cx: coxa, Eb: esclerito basal, Epi: episterno, Epim: epímero, Esc: escapo, Fe: fémur, Fla: flagelo, Fu: furcaesternum, Glo: glossa, La: labro, Md: mandíbula, Oc: ojo compuesto, Oce: ocelo, Of: órgano de fijación, Par: paraglossa, Plb: palpo labial, Pcl: postclipeo, Pe: pedicelo, Pn: pronoto, Prm: prementum, Sant: sutura antenal, Sco: sutura coronal, Se: sutura epistomial, Sf: sutura frontal, Sm: sutura medial, Ta: tarso, Ti: tibia, To: toruli, Tr: trocánter. Adaptadas de H. Aspöck et al. (1991).

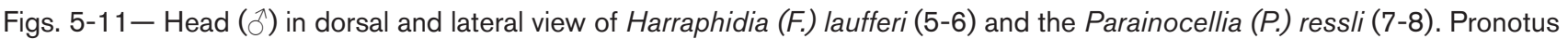
(ठ) in lateral (9), dorsal (10) and ventral (11) view of Harraphidia (F.) laufferi. Acl: anteclipeus, Apl: pleural apophise, Cl: clipeus, Cv: cervicalium, Cx: coxa, Eb: basal esclerite, Epi: episternum, Epim: epimere, Esc: scapus, Fe: femur, Fla: flagelus, Fu: furcaesternum, Glo: glossa, La: labrus, Md: mandible, Oc: compoust eye, Oce: ocelus, Of: Fix organ, Par: paraglosse, Plb: labial palp, Pcl: postclipeus, Pe: pedicellus, Pn: pronotus, Prm: prementum, Sant: antennal suture, Sco: coronal suture, Se: epistomial suture, Sf: frontal suture, Sm: medial suture, Ta: tarsus, Ti: tibia, To: toruli, Tr: trochanter. Adapted from H. Aspöck et al. (1991). 
y no existe arolio. El abdomen está formado por diez segmentos claramente separados, con estructuras genitales externas bien desarrolladas en $\operatorname{los} \hat{\delta} \hat{\sigma}$ (figs. 16, 43-79), y existencia de un largo ovopositor en las 9 우 (figs. 1-3, 17). La larva es depredadora de huevos y larvas de insectos y de pequeños artrópodos como ácaros, arañas, insectos, etc., y es de tipo campodeiforme, edáfica o corticícola (figs. 19-22). La pupa es activa, dectica y exarada (fig. 23).

Las rafidias son insectos que suelen pasar desapercibidos en la Naturaleza, al tratarse de un grupo de pequeño tamaño, poco llamativos y con escasa capacidad de vuelo. A esto se une que los individuos generalmente forman poblaciones reducidas que colonizan
12

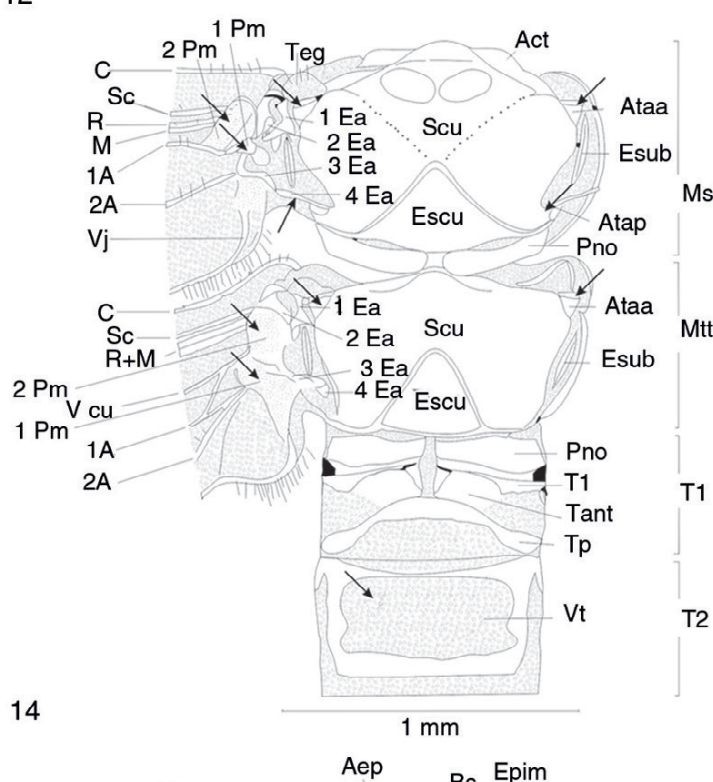

13

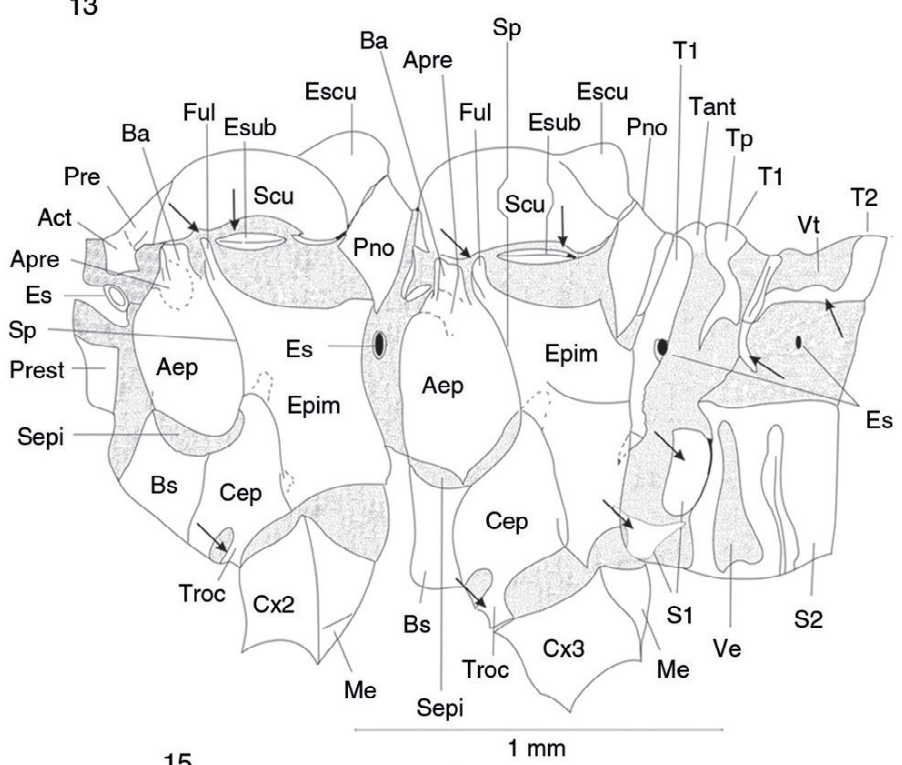

15
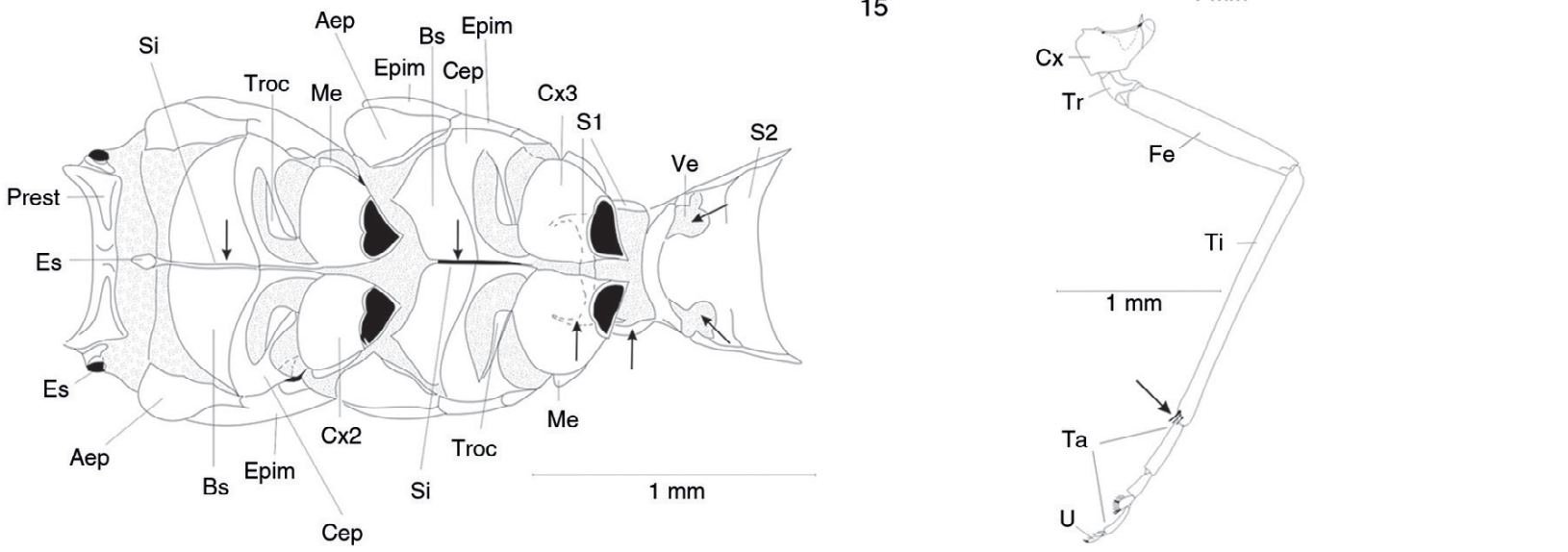

Figs. 12-15.- Harraphidia (F.) laufferi. Pterotórax y base del abdomen (§) en vista dorsal (12), lateral (13) y ventral (14). Pata

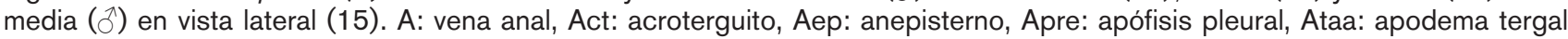
articular anterior, Atap: apodema tergal articular posterior, Ba: basialar, Bs: basisternum, C: vena costal, Cep: catepisterno, Cu: vena cubital, Cx: coxa, Ea: esclerito axilar, Eb: esclerito basal, Eba: esclerito basialar, Epim: epímero, Es: espiráculo, Escu: escutelo, Esub: esclerito subalar, Fepi: sutura episternal, Fi: fisura invaginada, Fp: fisura pleural, Ful: fulcro, M: vena media, Me: meron, Mst: mesotórax, Mtt: metatórax, Pm: placa mediana, Pn: pronoto, Pno: postnoto, Pre: prescutum, Prest: preesterno, Pte: pterale, R: vena radial, S: esterno, Sc: vena subcostal, Scu: scutum, Sepi: sutura episternal, Si: sutura invaginada, posterior, Sp: sutura pleural, T: tergo, Tant: terguito anterior, Teg: tégula, TI: terguito lateral, Tp: terguito posterior, Troc: trocantino, Ve: ventana esternal, Vj: vena jugal, Vt: ventana tergal. Adaptadas de H. Aspöck et al. (1991).

Figs. 12-15.- Harraphidia (F.) laufferi. Pterothorax and abdominal basis (đ) of dorsal (12), lateral (13) and ventral view (14). Middleg ( $\widehat{\jmath})$ in lateral view (15). A: anal vein, Act: acroterguite, Aep: anepisternum, Apre: pleural apophisis, Ataa: anterior tergal articular apodeme, Atap: posterior tergal articular apodeme, Ba: basialar, Bs: basisternum, C: costal vein, Cep: catepisternum, Cu: cubital vein, Cx: coxa, Ea: axilar esclerite, Eb: basal esclerite, Eba: basialar esclerite, Epim: epimerus, Es: spiracle, Escu: escutelus, Esub: subalar sclerite, Fepi: episternal suture, Fi: invaginated fisure, Fp: pleural fisure, Ful: fulcrum, M: median vein, Me: meron, Mst: mesothorax, Mtt: metathorax, Pm: median plate, Pn: pronotus, Pno: postnotus, Pre: prescutum, Prest: presternum, Pte: pterale, R: radial vein, S: sternum, Sc: subcostal vein, Scu: scutum, Sepi: episternal suture, Si: invaginated posterior suture, Sp: pleural suture, T: tergun, Tant: anterior terguite, Teg: tegula, Tl: lateral terguite, Tp: posterior terguite, Troc: trocantine, Ve: sternal window, Vj: jugal vein, Vt: tergal window. Adapted from H. Aspöck et al. (1991). 

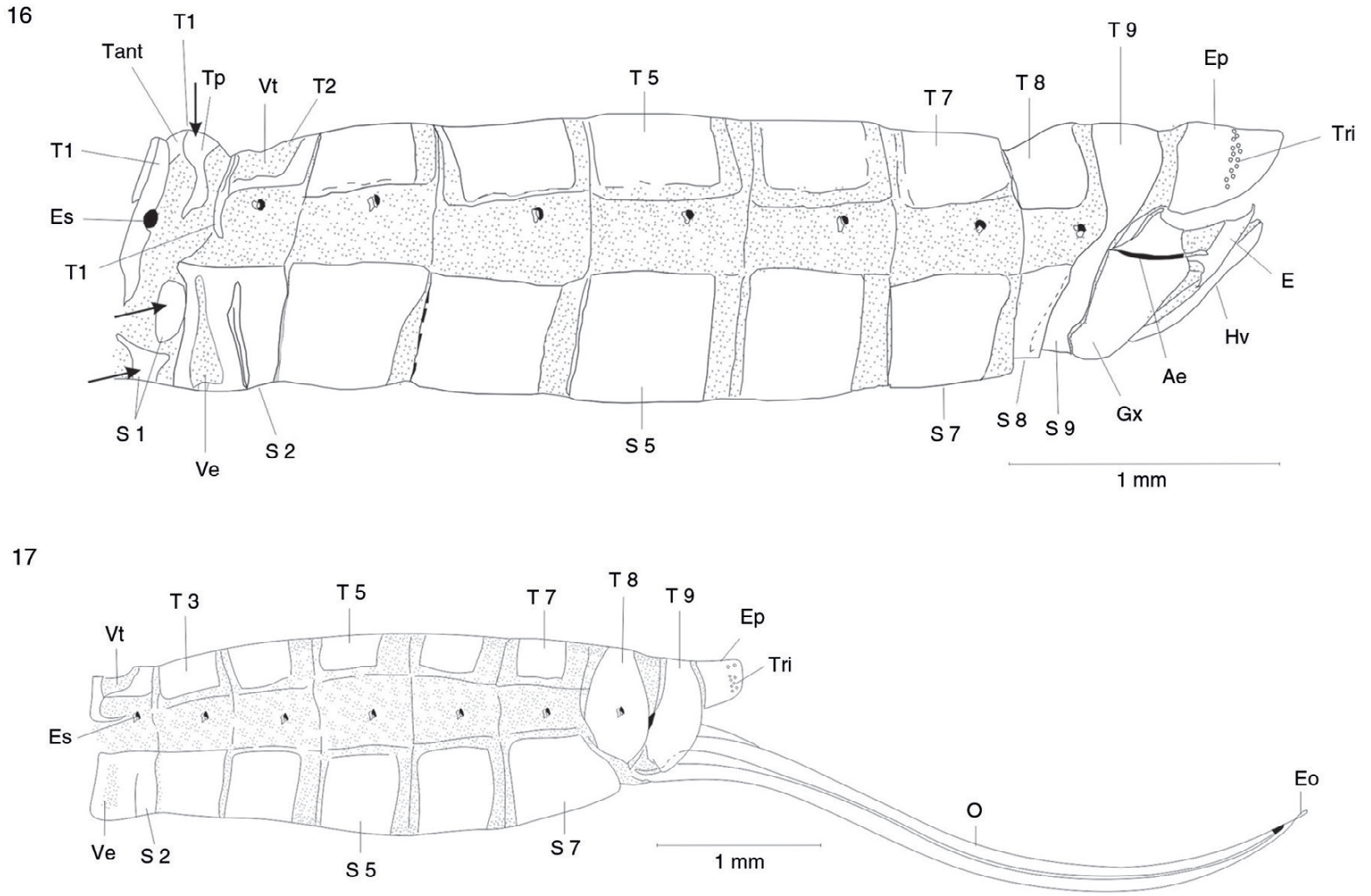

18

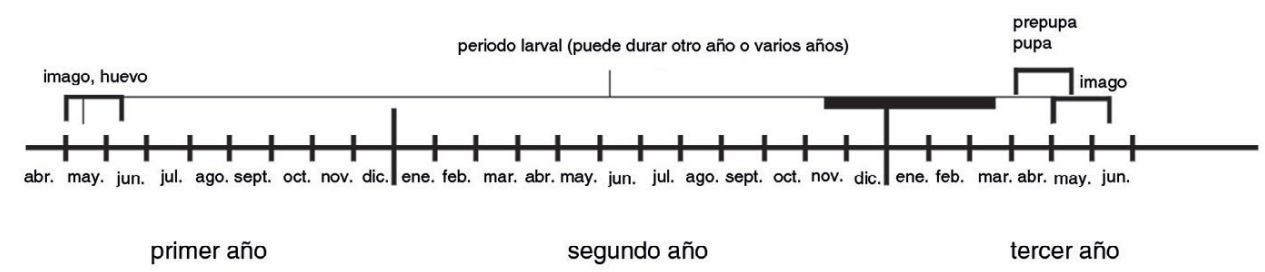

Tipo I: último estado de hibernación: larva, pupación en primavera, imagos: primavera-principios de verano

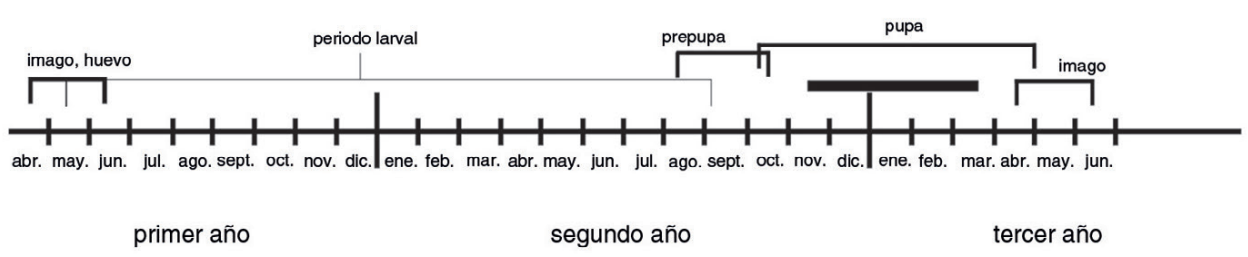

Tipo II: último estado de hibernación: pupa, pupación en verano u otoño, imagos: primavera del prox. año

Figs. 16-18.- Abdomen $\hat{\jmath}(16)$ y $q$ (17) en vista lateral de Harraphidia (F.) laufferi. Esquema de los tipos de ciclos biológicos existentes en los Raphidioptera ibéricos (18). Ae: apodema del estilo, E: estilo, Eo: estilo del ovopositor, Ep: epiprocto, Es: espiráculo, Gx: gonocoxito, Hv: hipovalva, O: ovopositor, S: esterno, T: tergo, Tant: terguito anterior, Tp: terguito posterior, Tri: tricobotrios, Ve: ventana esternal, Vt: ventana tergal. Figs. 16 y 17, adaptadas de H. Aspöck et al. (1991). Fig. 18, adaptada de H. Aspöck (2002).

Figs. 16-18.- Abdomen $\widehat{\partial}(16)$ and $+(17)$ in lateral view of Harraphidia (F.) laufferi. Scheme of biological cycle types in lberian Raphidioptera (18). Ae: stilus apodeme, E: stilus, Eo: ovipositor stylus, Ep: epiproct, Es: spiracle, Gx: gonocoxite, Hv: hipovalve, O: ovopositor, S: sternum, T: tergum, Tant: anterior tergite, Tp: posterior tergite, Tri: trichobotria, Ve: esternal window, Vt: tergal window. Figs. 16 and 17, adapted from H. Aspöck et al. (1991). Fig. 18, adapted from H. Aspöck (2002). 
áreas muy localizadas y con fenologías muy reducidas a lo largo del año, generalmente primaverales. Por su característico "cuello" (figs. 1-4), en España, en lenguaje castellano popular, son conocidos como "moscas jirafa" o "moscas serpiente".

Los rafidiópteros constituyen, por diversas razones, uno de los grupos de insectos más interesantes y peculiares. Están considerados, dentro de los insectos holometábolos vivientes, como uno de los grupos conocidos más antiguos y primitivos, y por tanto son considerados como auténticos "fósiles vivientes" (Thenius, 2000).

Su historial paleontológico es extenso. Descartadas las familias fósiles (Paleozoico) inicialmente asignadas a este orden y hoy transferidas a otros órdenes (Orthoptera, Grylloblattaria y Protorthoptera), el orden Raphidioptera parece tener origen en el Triásico, e incluso probablemente en el Pérmico, aunque su diversificación se produjo sin duda en el Mesozoico, y posee registro de numerosas familias extintas en sedimentos y ámbar del Jurásico Inferior al Cretácico Inferior, Medio y Superior de Europa, Asia, Norteamérica y Sudamérica (†Priscaenigmatidae, $\uparrow$ Baissopteridae, $\uparrow$ Alloraphidiidae, $\uparrow$ Mesoraphidiidae, etc.). Más abundante es la fauna del Cenozoico, con numerosos registros en ámbar y rocas sedimentarias (Eoceno, Oligoceno y Mioceno de Europa y Norteamérica), donde ya hallamos las relictas familias actuales (Inoceliidae, Raphidiidae), siendo de destacar el registro fósil en la Península Ibérica (Pérez de la Fuente et al., 2012). Más reciente información sobre este particular en Wang (1987), Hong \& Chang (1989), Nel et al. (1990), Oswald (1990), H. Aspöck et al. (1991), Martins-Neto \& Vulcano (1992), Martins-Neto \& Nell (1992), Hong (1992), Ren \& Hong (1994), Ren (1994, 1995, 1997), Engel (1995, 2002, 2003, 2011), U. Aspöck \& H. Aspöck (1999a), Grimaldi (2000), Grimaldi et al. (2002), Grimaldi \& Engel (2005), Perrichot \& Engel (2007), Jepson \& Jarzembowski (2008), Engel \& Grimaldi (2008), Jepson et al. (2009, 2012), Liu et al. (2013), Makarkin \& Archibald (2014), Makarkin \& Khramov, (2014), Liu et al. (2014b), etc.

Aunque existen registros fósiles en Sudamérica (Cretácico Inferior) y en zonas de pretéritos ambientes tropicales, la distribución actual del orden Raphidioptera se limita a la región Holártica. En la Región Paleártica abarcan la mayoría de las zonas arbóreas cuyas fronteras meridionales se sitúan en las cadenas montañosas del norte de África, en Israel, en el norte de Irak, Irán, Pakistán, India, Myanmar, el norte de Tailandia y en las montañas de Taiwán ( $\mathrm{H}$. Aspöck et al., 1998b; Liu et al., 2014a). En la Región Neártica el área de distribución se extiende desde el sur de Canadá y oeste de Estados Unidos hasta México. Curiosamente el orden Raphidioptera no está actualmente representado en las regiones tropicales húmedas, ni en todo el hemisferio sur, probablemente debido al cambio climático en la transición EocenoOligoceno (Grimaldi \& Engel, 2005; Engel \& Grimaldi, 2008), aunque otras teorías han sido propuestas (H. Aspöck, 1998b).

\section{Datos Generales SOBRE SU BIOLOGÍA, COMPORTAMIENTO Y CICLOS BIOLÓGICOS}

Sobre su biología anotemos que los imagos son insectos de actividad diurna y muy heliófilos (figs. 1-4), con tiempo frío o nublado reducen notablemente su actividad. Se mueven mucho por la vegetación, pero vuelan poco y en cortos trayectos. Por la noche son totalmente inactivos $\mathrm{y}$, en un principio, no son atraídos por fuentes de luz artificial. Son insectos a veces abundantes, pero poco frecuentes, pues el periodo en estadio de imago, en comparación con el de larva, es muy corto, en cautividad alcanzan longevidades de dos semanas, pero en la naturaleza es mucho menor. La mayoría de los rafidiópteros tienen su fase de imago a finales de la primavera o principios de verano, y sólo a mayores altitudes aparecen ya entrado el verano. Son generalmente estenotópicos, a veces vinculados a un determinado sustrato o medio, y por ello los imagos a veces se concentran en zonas muy pequeñas, $y$ en la gran mayoría de las áreas solo aparecen una o muy pocas especies distintas. A veces presentan cierta capacidad de agregación, concentrándose numerosos ejemplares en muy pequeño espacio. Las especies de Raphidiidae son depredadores generalistas de pequeños artrópodos, la mayor fuente de alimentación la constituyen los pulgones y otros pequeños insectos fitófagos o corticícolas, también en ocasiones ingieren polen (H. Aspöck, 2000). Existe el canibalismo, y en ocasiones las $q+$ en cautividad devoran sus propios huevos recién puestos. En las especies de Inocelliidae, aunque ocasionalmente se ha encontrado polen en su tubo digestivo (H. Aspöck et al., 1991, 2012), se considera que la fase adulta no es depredadora y/o no se alimenta. Diversos parásitos y parasitoides han sido citados (Achtelig, 1974, 1975a; H. Aspöck et al., 1991; H. Aspöck, 2002), y en el caso de nuestras especies se citarán en cada caso.

En la bibliografía no existen muchos datos sobre el comportamiento de los individuos durante el cortejo y la cópula. Las observaciones proceden del periodo entre los años 1934 y 1966 de Kästner (1934), Eglin (1939), Zabel (1941), Acker (1966), resumidos en H. Aspöck \& U. Aspöck (1971a). H. Aspöck et al. (1991) y U. Aspöck et al. (1994a). Hacen referencia a especies paleárticas y neárticas de la familia Raphidiidae como Phaeostigma (Ph.) notatum, Ph. holzingeri, Xanthostigma xanthostigma, Ulrike attica, Raphidia (R.) ophiopsis, Agulla (Agulla) bicolor, A. (A.) astuta y A. (Glavia) adnixa, así como alguna especie paleártica de la familia Inocelliidae, Inocellia crassicornis y Parainocellia (Parainocellia) braueri. El comportamiento de A. bicolor durante la cópula también es motivo de estudio en Kovarik et al. (1991). 
En el cortejo previo existen similitudes entre las dos familias, pero se considera confirmado que las familias Raphidiidae e Inocelliidae presentan claras diferencias en el comportamiento durante el apareamiento y la cópula (H. Aspöck et al., 2012). Hay que destacar la presencia en algunas especies como Parainocellia braueri de un órgano de fijación en la base de las antenas con el cual el $\hat{\sigma}$ durante la cópula fija su cabeza a los segmentos ventrales de la q (U. Aspöck et al., 1994a).

Como insectos depredadores, el apareamiento en rafidiópteros es una combinación de atracción y agresión que se da por parte de ambos sexos, y especialmente en la $q$ se produce un comportamiento agresivo consistente en intentos de morder a su pareja. Las 9 ㅇ son sobre todo bastante agresivas, pudiendo incluso llegar a matar al $\hat{\partial}$. La búsqueda de pareja y el cortejo se caracterizan en ambos sexos por la excitación que consiste en activos movimientos hacia delante y detrás, limpieza de las antenas, de la parte anterior de la cabeza, así como las patas posteriores. Existen movimientos de aseo que consisten en pasar la tibia y el tarso del primer par de patas por las piezas bucales, lo que permite que estas estructuras, una vez limpias, sirvan como herramientas de aseo para las antenas. Este comportamiento queda desencadenado mediante un previo contacto visual, pero también gracias a la secreción de feromonas de carácter sexual. La existencia de quimiorreceptores, tanto en los tarsos como en las antenas, posibilita la localización no visual del $\delta$ por parte de la $O$. Acker (1966) ha comprobado que se consigue estimular estos movimientos en un $\delta$ que ha sido introducido en un recipiente de cristal, donde instantes antes había estado una ${ }^{+}$.

En el momento en el que los individuos de ambos sexos se visualizan mutuamente, se establece un punto crítico ya que un individuo tiene que reconocer al otro como pareja copuladora y no como un posible depredador. Si el acercamiento se produce demasiado deprisa, el contrario es atacado. Los movimientos de cortejo tienen un desarrollo diferente en los dos sexos. Los $\widehat{\partial} \widehat{\jmath}$ mueven la cabeza, reacción que no ha sido observada en las $q$ 우. En ambos sexos las piezas bucales se abren y se cierran y las antenas son movidas con vehemencia. Estos comportamientos son interrumpidos en el momento en el que surgen reacciones agresivas, que por otra parte provocan respuestas defensivas. Posteriormente se reanuda el cortejo.

El movimiento excitado de las antenas y de la

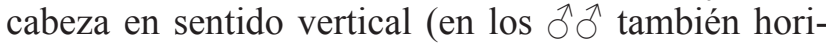
zontal) indica las fases previas a la cópula. Es interesante observar el grado de relación que existe entre los comportamientos alimenticios y los de cortejo, y cómo ambos se solapan. Otra señal característica que se produce durante el cortejo de los rafidiópteros, pero exclusivamente en los $\hat{\partial} \delta^{\lambda}$ de la familia Raphidiidae, es un movimiento rotacional del abdomen. En ambas familias y en ambos sexos se produce un movimiento vertical de vibración del abdomen. Rupprecht (1975) y Devetak (1992a) han observado comportamientos parecidos en otros grupos mejor estudiados de de Neuroptera, como son las familias Sialidae o Chrysopidae, en las cuales también se producen movimientos rítmicos del tagma posterior, que producen vibraciones que pasan a través de las patas al sustrato y que son recogidas mediante específicos órganos receptores. Las $O+q$ de ambas familias serpentean con el ovopositor. Kovarik et al. (1991) describen otros movimientos parecidos que conducen a la secreción de sustancias a través del extremo final del abdomen. Esta zona se sitúa a la altura del pterostigma cuando las alas se encuentran en reposo, donde dichas sustancias son almacenadas y posteriormente son distribuidas mediante un movimiento alar, ya que en todos los individuos de la familia Raphidiidae observados se detecta un balanceo de las alas de uno o ambos lados.

Cuando finalmente predominan los componentes sexuales, se llega a un contacto directo entre los miembros de la pareja con las cabezas y las antenas. $\mathrm{El} \partial$ toca con las patas las alas de la + o con las antenas el ovopositor. Con ello se puede iniciar la cópula propiamente dicha.

En las distintas especies, existen grandes diferencias, al menos en la familia Raphidiidae y probablemente también en Inocelliidae, durante las fases precopulatorias. La disponibilidad a la cópula en la + parece estar relacionada con la maduración de los óvulos y suele manifestarse, como muy pronto, una semana después del paso a imago en el individuo, aunque se contradice con investigaciones en laboratorio que han demostrado lo contrario. En condiciones de laboratorio solo se producen emparejamientos con $+9+$ muy jóvenes (de uno a tres días), los $\widehat{\partial} \hat{o}$ al contrario eran de más edad. El $\widehat{\partial}$ se encuentra sexualmente disponible mucho antes, pero apenas se conocen datos sobre la edad mínima que necesitan tener los $\widehat{\partial} \widehat{\partial}$ para ser aptos para la cópula.

En la mayoría de las especies, al presentarse comportamientos agresivos, muchos intentos de cópula acaban con la muerte de uno de los dos miembros de la pareja, que frecuentemente suele ser el $\widehat{\partial}$. Algunas especies, en cambio, no presentan en absoluto un carácter agresivo, como parece ser el caso de Ulrike attica (H. Aspöck \& U. Aspöck, 1967).

Observaciones de U. Aspöck et al. (1994a) indican claramente que existen dos tipos de posiciones de cópula. Una es la llamada "posición de tandem" y la otra es la "posición de arrastre". La posición de tandem parece ser la más habitual dentro de la familia de Inocelliidae, comienza cuando el $\widehat{\partial}$ se coloca debajo de la ${ }_{\text {+ }}$, desde detrás o desde delante girando posteriormente. El $\delta$ alcanza de esta forma con su cabeza los esternitos centrales de la $P$ y dobla su abdomen hacia arriba hasta que se establece contacto entre las regiones ventrales de las genitalias. Los dos individuos unidos mediante dos puntos se mueven de forma 
sincronizada como un tandem. En Parainocellia (P.) braueri se ha observado como emergen unos sacos desde la cara interior de la base de las antenas del $\widehat{\partial}$, que son usados como órganos de fijación, y que durante la cópula entran en contacto con el $5^{\circ}$ esternito del abdomen de la $q$. Si se produce una fijación por adhesión o succión se desconoce. Dada la morfología de la base de las antenas de los Inocelliidae (donde el abombamiento es más prominente en los $\widehat{\partial} \widehat{\gamma}$ que en las $ㅇ$ ) se intuye que todos ellos tienen el citado órgano de fijación. Zabel (1941) expone que el

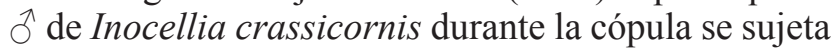
a la $\bigcirc$ con ayuda de sus mandíbulas (probablemente se trate de un error de observación, ya que dicha especie también posee un órgano de fijación).

También la familia Raphidiidae normalmente inicia la cópula en "posición de tandem", pero tras pocos pasos, el $\widehat{\partial}$ es catapultado hacia atrás apoyándose con el dorso sobre el sustrato. A esta situación se la denomina "posición de arrastre", y es típica de la familia Raphidiidae. La duración de la cópula es más larga en Inocelliidae pudiendo llegar a las tres horas. En Raphidiidae va desde los pocos minutos hasta la hora y media. Es de suponer que pueda existir una relación entre la mayor duración del proceso en Inocelliidae con la doble fijación existente entre los miembros en esa familia. Zabel (1941) observó en una pareja de Raphidia (Raphidia) ophiopsis una doble cópula, en donde la primera duró veinticinco minutos y la segunda diez minutos existiendo entre ambas un intervalo de cinco días. Se desconoce si es habitual que se sucedan dos o incluso más cópulas. Una segunda cópula ha sido también observada en las especies Xanthostigma xanthostigma, Ulrike atica, Raphidia ophiopsis y Parainocellia (P.) braueri (U. Aspöck et al., 1994a).

La trasferencia de esperma en algunas especies es mediante el concurso de espermatóforo. La primera vez que se hace referencia a la existencia de un espermatóforo es respecto a una especie mejicana perteneciente al género Agulla Navás, 1914. Los autores americanos Acker (1966) y Kovarik et al. (1991) los denominan "white objects" pero sin detallar. Posteriormente se vuelve a citar en especies del género Alena Navás, 1916. No existen referencias sobre la existencia de espermatóforos en especies paleárticas. En Inocelliidae no hay pruebas de la existencia de un espermatóforo. Las q 0 en su comportamiento tras la cópula no realizan rituales de limpieza, búsqueda o ingesta de alimento que puedan indicar la existencia de un espermatóforo.

La vida del imago en el caso de los $\widehat{\partial} \widehat{\partial}$ es corta, ya que mueren tras la cópula, y por tanto viven solo unos días. Las $ᄋ$ $O$ fecundadas realizan la puesta pocos días tras la cópula y mueren tras realizar la puesta, por tanto su vida de imago es de pocas semanas. Estos periodos de tiempo pueden variar dependiendo del clima (más o menos favorable) y de la cantidad de comida ingerida. Como se indica en H. Aspöck et al. (1991) estos factores pueden condicionar el momento de la cópula y la puesta de los huevos. La vida del imago es, en condiciones de laboratorio, de varias semanas (hasta dos meses), sobre todo si se trata de animales que aún no han copulado y si se les abastece con suficientes cantidades de alimento fresco.

Tras la cópula, la puesta de huevos comienza, en condiciones experimentales, desde pocas horas a varios días, pudiéndose prolongar durante unos pocos días a semanas. Los sustratos elegidos suelen ser las cortezas y otras partes porosas de la vegetación, como por ejemplo madera en descomposición, en donde introducir los ovopositores/ huevos. Woglum \& McGregor (1959) han encontrado puestas incluso en tegumentos de cóccidos muertos. El número total de huevos depositados por una $q$ se cuenta por centenas (hasta más de doscientos). Los huevos son alargados, cilíndricos, algo curvados y con uno de los extremos es algo más afilado, y son de coloración amarillenta con un gran micropilo. Según las especies su longitud comprende entre 1.2 y $1.7 \mathrm{~mm}$, y entre 0.25 a $0.5 \mathrm{~mm}$ de anchura. El porcentaje de huevos no fecundados es variable, suele ser alto, lo cual se puede interpretar como un sistema de defensa contra los depredadores. El estadio de huevo en la mayoría de las especies estudiadas, se mantiene desde escasos días hasta un máximo de tres semanas. Pocos días antes de la eclosión, la larva non nata ya realiza movimientos de cabeceo dentro del huevo. Finalmente se rompe el corion en forma de un gran surco por el cual emerge la larva en pocos minutos.

El tiempo de desarrollo desde el huevo hasta el imago presenta diferencias a nivel de familia, géneros o incluso especies, habiendo cierta flexibilidad y dependiendo también de factores externos y la alimentación disponible. Desarrollos de un año son la excepción, siendo la media de dos años. En Inocelliidae el desarrollo es aún más largo, estando el mínimo en tres años, pudiendo llegar a los siete años (H. Aspöck et al., 2012). Desarrollos de tres años se han estudiado en los géneros Raphidia, Phaeostigma Navás, 1909, Subilla, Ohmella H. Aspöck \& U. Aspöck, 1968 o Africoraphidia H. Aspöck \& U. Aspöck, 1969. Desarrollos de cuatro años se han observado en especies de Phaeostigma y de cinco años, junto y excepcionalmente a otros de tres años, en Ohmella postulata H. Aspöck \& U. Aspöck, 1977. En algunas especies se ha observado incluso seis años y más. Según las condiciones, la fase de desarrollo oscila bastante, no sólo dentro de una misma especie sino también dentro de una misma puesta.

Las larvas son campodeiformes (figs. 19-22), y al contrario que los imagos, evitan la luz intensa y viven por lo general ocultas en las grietas de las cortezas o son edáficas. En las especies de ambas familias son depredadoras generalistas, y se alimentan principalmente de artrópodos de cuerpo blando (larvas de lepidópteros, 
19

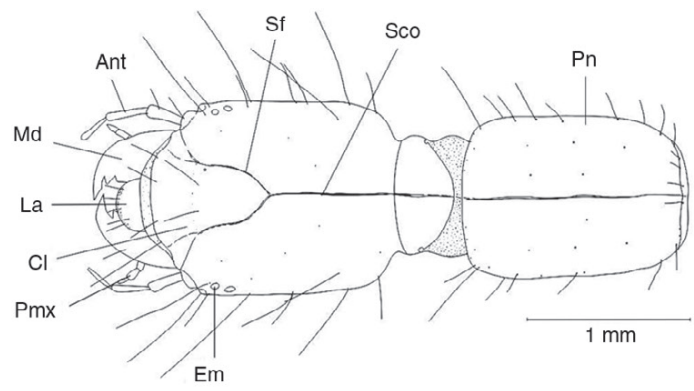

21

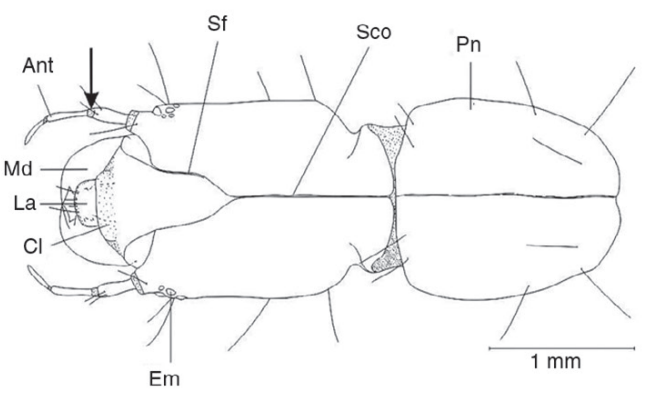

20

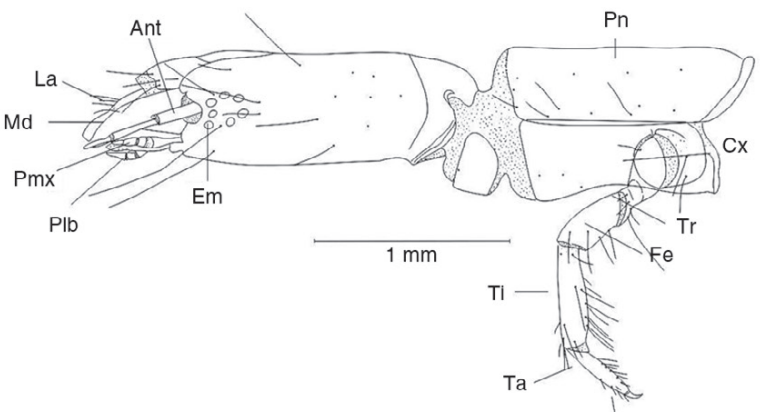

22

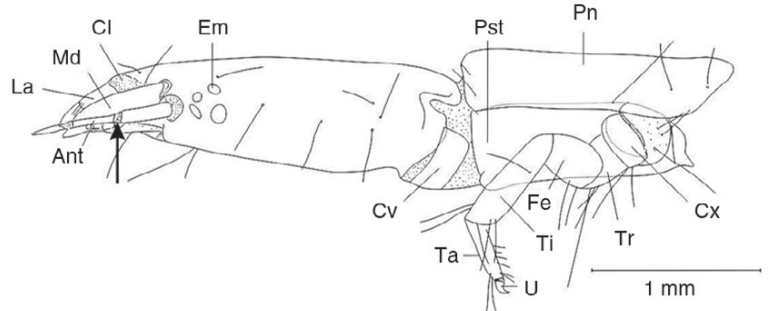

23

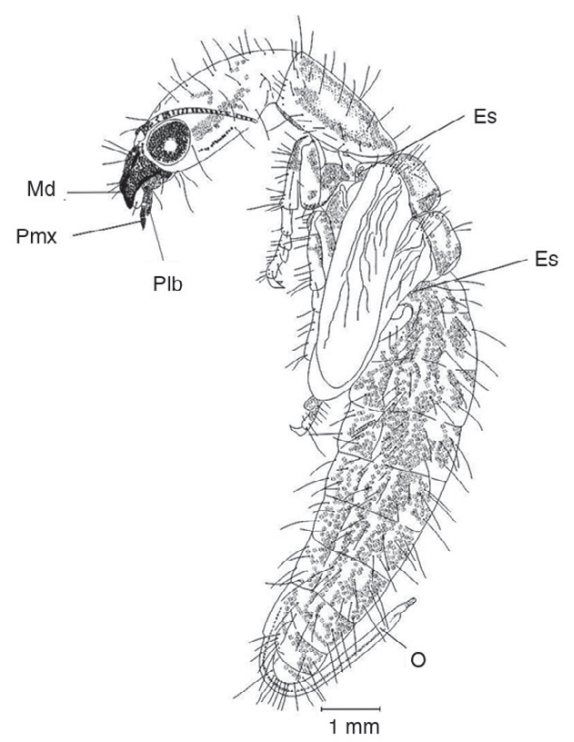

Figs. 19-23.- Larva en vista dorsal y lateral de Phaeostigma (C.) klimischiella (19-20) y de Parainocellia (P.) ressli (21-22). Pupa (ㅇ) de Phaeostigma (M.) mayor (23). Ant: antena, Cl: clípeo, Cv: cervicalium, Cx: coxa, Em: estemata, Es: espiráculo, Fe: fémur, Ft: fosa tentorial, La: labro, Md: mandíbula, Mx: maxila, O: ovopositor, Plb: palpo labial, Pmx: palpo maxilar, Pn: pronoto, Pst: proesterno, Sco: sutura coronal, Sf: sutura frontal, Sgu: sutura gular, Ta: tarso, Ti: tibia, Tr: trocánter, U: uña. Adaptadas de H. Aspöck et al. (1991).

Figs. 19-23.- Larva in dorsal and lateral view of Phaeostigma (C.) klimischiella (19-20) and Parainocellia (P.) ressli (21-22). Pupa () of Phaeostigma (M.) mayor (23). Ant: antenna, Cl: clipeus, Cv: cervicalium, Cx: coxa, Em: stemmata, Es: spiracle, Fe: femur, Ft: tentorial fossus, La: labre, Md: mandible, Mx: maxilla, O: ovopositor, Plb: labial palp, Pmx: maxilar papl, Pn: pronotus, Pst: proesternus, Sco: coronal suture, Sf: frontal suture, Sgu: gular suture, Ta: tarsus, Ti: tibia, Tr: trochanter, U: snail. Adapted from H. Aspöck et al. (1991).

coleópteros e himenópteros, pequeñas arañas, psocópteros, áfidos, huevos de otros insectos, etc.). También se ha observado que las larvas pueden estar sin ingerir alimento durante varias semanas o meses. Como uno de sus caracteres más primitivos, suelen pasar por una media de diez estadios larvarios (hasta 16), al menos a lo largo de dos años, antes de que se produzca la pupación (H. Aspöck, 2003). El último estadio larvario busca un lugar protegido en una cavidad de la corteza o en otros sustratos adecuados, y entra en fase de 
prepupa, fase que se prolonga entre una y dos semanas, y finaliza mediante una última muda, alcanzando el estadio de pupa (fig. 23). Las pupas son exaradas y decticas, y el estadio de pupa tiene una duración de entre diez y veinte días, habiendo poca variabilidad a nivel de especie. El abandono del espacio pupal se realiza preferentemente en horas matinales (H. Aspöck et al., 1991). Tras trepar por una superficie vertical, estirar y endurecerse las alas, comienza la actividad del imago.

Ya que lo mencionaremos para cada una de las especies ibéricas, conviene citar los diferentes tipos de ciclos vitales que en ellas se distinguen, dependiendo de en qué estación del año ocurra la pupación (fig. 18).

CICLO TIPO I: En la mayoría de las especies de ambas familias. El último invierno transcurre en fase de larva. La pupación sucede durante la primavera y esta fase se prolonga desde unos cuantos días hasta 3 semanas. Los adultos emergen en primavera o principios del verano.

CICLO TIPO II: En algunas (o todas) las especies de algunos géneros de Raphidiidae, como por ejemplo: Atlantoraphidia, Harraphidia, Hispanoraphidia u Ohmella. El último invierno transcurre en fase de pupa. La pupación normalmente ocurre durante el verano u otoño, y la fase de pupa se prolonga varios meses (hasta 10) y los adultos emergen en la primavera del siguiente año.

Por tanto en nuestras especies el periodo de hibernación suele ocurrir normalmente en los últimos estadios larvarios (ciclo tipo I) o en la fase de pupa (ciclo tipo II), y nunca en fase de huevo o adulto.

BREVE HISTORIAL SOBRE SU CONOCIMIENTO EN LA Península IbÉRICA

Los primeros rafidiópteros fueron citados en Europa en el siglo XVI. Tras las primeras descripciones (Linnaeus, 1735, 1758; Rösel von Rosenhof, 1755; Fabricius, 1781; Latreille, 1798; Stephens 1829, 1836; Burmeister 1829, 1837, 1839; Schummel, 1832), es Rambur (1842) el que cita por primera vez un rafidióptero en la Península Ibérica, apareciendo con el tiempo algunos trabajos posteriores sobre la fauna Ibérica (Rosenhauer 1856, Pictet 1865; Hagen 1866; McLachlan 1902), y a su vez las primeras monografías sobre el orden (Schneider, 1843; Albarda, 1891).

No sería posible concebir el estudio de los neurópteros en general, y de los rafidiópteros de España y Portugal en particular, sin las numerosas publicaciones del autor español Longinos Navás (1858-1938), quien durante casi cuarenta años centró sus estudios en la taxonomía y sistemática de este grupo de insectos. En ellos se describen numerosos nuevos taxa y se incluyen multitud de citas de rafidiópteros en nuestra fauna, aportando sus dos conocidas monografías para la Península Ibérica (Navás 1904, 1905a, 1905b, 1905c, 1905d, 1908, 1909a, 1910, 1913a, 1914a, 1914b, 1914c, 1915a, 1918a, 1920, 1921, 1923, 1924, 1928a). Lamentablemente los criterios taxonómicos seguidos por él han resultado ser de escaso valor y rigor científico, y sus identificaciones han sido frecuentemente puestas en duda, ya que mayoritariamente en sus identificaciones no tenían en cuenta la genitalia, y basaba sus estudios casi exclusivamente en caracteres como la venación alar (en gran parte muy variable), y por ello sus trabajos consisten en un compendio de taxa nuevos, que más que un avance, aportaron mucha confusión, y por ello su obra ha sido permanentemente motivo de controversia. Piénsese que sólo de estos tres órdenes de insectos (Neuropterida) describió aproximadamente 375 géneros, 2.173 especies y 301 variedades (Monserrat, 1986b) del total de 388 géneros y 2.684 especies por él descritos de diferentes órdenes de insectos y arácnidos (Monserrat, 1985, 1986b, 2011; Bastero Monserrat, 1989). De todos estos taxa, multitud de géneros, y nada menos que 152 especies y 149 variedades de Neuropterida fueron descritas por él en la Fauna Ibero-Balear, que sumadas a las descritas de las Islas Canarias alcanzan un total de 239 taxa: 4 de Megaloptera y 208 de Neuroptera ( +15 de Canarias), y en lo que nos compete, 12 de Raphidioptera (Monserrat, 2011). Por tanto, el gran legado de Navás ha quedado reducido realmente a alguna especie descrita que ha mantenido su validez taxonómica y a ciertos datos sobre la distribución geográfica de algunas especies. Desafortunadamente son solo sus publicaciones las que nos quedan, ya que su colección privada sufrió diversas y penosas vicisitudes a lo largo de los años (Monserrat 1985, 1986b), y por tanto, los ejemplares con los que contamos actualmente depositados en instituciones para revisar su labor son proporcionalmente muy escasos.

A partir del artículo de M. M. Principi (Principi, 1960), y en especial desde el año 1965, son los autores Horst Aspöck, Ulrike Aspöck y Herbert Rausch los que han dedicado gran parte de su trabajo al estudio de los rafidiópteros, y han aportado multitud de datos sobre este orden de insectos, que tienen como fruto la revisión de la fauna de rafidiópteros de la Región Neártica (U. Aspöck, 1974), la revisión de las especies de Europa (H. Aspöck et al., 1980a), y la revisión del orden a nivel mundial (H. Aspöck et al., 1991), recopilando los conocimientos sobre taxonomía, biología, ecología, registros fósiles, filogenia y distribución que se tiene hasta el momento, con nuevas aportaciones y puesta al día en relación a la Región Paleártica occidental (H. Aspöck et al., 2001), e incluyendo a lo largo de su dilatada labor numerosos datos referentes a la fauna de la Península Ibérica, describiendo taxones nuevos, aportando multitud de datos sobre otras especies (H. Aspöck \& U. Aspöck 1968a, 1977), e intentando crear una clasificación solvente $(\mathrm{H}$. Aspöck \& U. Aspöck 1968b, 1968c, 1971b, 1973b, 1975; Popov, 1974). 
En lo que respecta a la Península Ibérica (hasta el presente, no existe ningún elemento citado en las Islas Baleares), este orden no es especialmente rico en especies, al menos en comparación con otros países mediterráneos (H. Aspöck et al., 1980a, 1991, 2001), estando representado por 16 especies. Curiosamente, la mayoría (10 de 16) de las especies habitantes en la Península Ibérica presentan una distribución circunscrita a sus dos tercios meridionales (figs. 162-169, 171), salvo una especie del género Atlantoraphidia: A. maculicollis (Stephens, 1836), dos del género Xanthostigma: X. aloysianum (Costa, 1855) y X. corsicum (Hagen, 1867), dos del género Phaeostigma: $P$. notatum (Fabricius, 1781) y $P$. italogallicum (H. Aspöck \& U. Aspöck, 1976), y una del género Venustoraphidia: $V$. conviventibus Monserrat \& Papenberg, 2012, en su mayor parte citadas o recolectadas en su tercio septentrional, generalmente en base a muy escasos ejemplares (figs. 156, 157, 159-161, 170). Este hecho sugiere un origen faunístico mayoritariamente norte-africano de este grupo de insectos en la fauna ibérica (Monserrat \& Papenberg, 2006, 2010, 2012), con escasos elementos más recientes de origen europeo que han ido ingresando a través de los Pirineos y han ido colonizando su región septentrional de influencia eurosiberiana.

A pesar de toda la información publicada hasta la fecha, y en lo que respecta a la Fauna Ibérica, el orden Raphidioptera no ha sido objeto de una investigación profunda basada en todo el estudio del material del que disponemos en la actualidad, y son proporcionalmente escasas las citas más recientes existentes sobre su fauna. Únicamente ha sido publicada últimamente la revisión de tres géneros de rafidiópteros ibéricos (Harraphidia, Phaeostigma, Venustoraphidia) con la descripción de nuevas especies (Monserrat \& Papenberg, 2006, 2010, 2012), y más recientemente Monserrat \& Triviño (2013) han recopilado una lista de los taxa ibéricos (nombres válidos, sinonimias y diferentes combinaciones nomenclaturales) registrados en la bibliografía, y en base a las citas fiables existentes en la bibliografía y a material inédito, aportan un cartografiado de la distribución de las especies ibéricas poniendo en evidencia que existen enormes áreas aún no prospectadas, y es de esperar que nuevas sorpresas nos depare el futuro. Por otra parte, toda la información existente sobre este orden de insectos en la península está muy dispersa o aún es desconocida, y es de gran importancia subsanar dicha laguna efectuando una revisión completa del orden en la Península Ibérica, con claves de taxa en castellano, que es lo que pretendemos en la presente contribución.

En ella se realiza una revisión del orden Raphidioptera de la Península Ibérica, que incluye la recopilación de toda la información bibliográfica existente relacionada con los taxa de su fauna, con la inclusión de todas las referencias existentes sobre sus géneros y especies, sobre sus sinonimias comúnmente aceptadas y sobre las diferentes combinaciones nomenclaroriales bajo las cuales han sido citadas en la bibliografía, aportando el listado de las especies conocidas en ella, así como una clave de identificación de las familias, géneros y especies ibéricas, y de cada una de ellas, anotamos su descripción morfológica, variabilidad hallada y los datos conocidos sobre sus estadíos juveniles, cuando consten, así como toda la información existente sobre su biología, distribución geográfica, fenológica y altitudinal.

\section{Material y métodos}

Para la revisión del orden Raphidioptera de la Península Ibérica se ha estudiado el material inédito que hemos recolectado en las últimas cuatro décadas, así como otro material, inédito o ya citado, existente en las diferentes colecciones entomológicas, cuyas siglas utilizadas se anotan a continuación:

\section{EEZ Estación Experimental del Zaidín (Granada, España) \\ FCTUC Universidad de Coimbra (Coimbra, Portugal) \\ MNCN Museo Nacional de Ciencias Naturales (Madrid, España) \\ MNHN Muséum National d'Histoire Naturelle (París, Francia) \\ NHM Natural History Museum (Londres, Inglaterra) \\ UAM Universidad Autónoma de Madrid (Cantoblanco, España) \\ UNIL Universidad de León (León, España) \\ USAL Universidad de Salamanca (Salamanca, España) \\ VM Colección Víctor J. Monserrat, Universidad Complutense (Madrid, España)}

Respecto a la información bibliográfica existente en relación a la fauna de rafidiópteros de la Península Ibérica, ahora compilamos toda la información histórica, tanto la general para el orden Raphidioptera, como para la de las dos familias que lo integran (Raphidiidae, Inocelliidae), junto con la existente sobre los géneros y las especies ibéricas conocidas en su fauna, y de ellas tanto en la denominación actualmente asumida, como de sus sinonimias actualmente aceptadas y de las diferentes combinaciones nomenclaturales bajo las que han sido citadas. Esta información afecta tanto a la específicamente relacionada con la Península Ibérica, como toda la existente, donde el lector puede recabar información adicional y complementaria sobre cada uno de los taxa ibéricos.

Estas reseñas se recopilan, cronológicamente ordenadas, anotando los autores de estas citas, su fecha de publicación y, para mayor comodidad del lector, la página donde se aporta información sobre sus alas (a), anatomía (anat), biogeografía (biogeo), biología (bio), inclusión en claves (c), inclusión en catálogos (cat), citología (cit), descripción original (d), distribución 
geográfica (dis), ecología (eco), etología (eto), fenología (fe), filogenia (f), fisiología (fis), generalidades (gen), histología (hist), huevo (h), identificación errónea (ie), larva (1), listado (1st), monografía (mon), morfología (mf), nomenclatura (nom), paleontología (paleo), parasitismo (pa), patología (patol), pupa (p), referencia (rf), sinonimia (sin), taxonomía (tx), técnicas $(\mathrm{t})$, genitalia del macho $(0)$ o genitalia de la hembra (q). En algunos casos conocemos la existencia de la información aportada, mas no disponemos de la publicación original o de su copia, y no nos ha sido posible anotar la/s página/s donde ésta ha sido anotada. En cualquier caso, la inclusión de estas citas es informativa, y no siempre implica la total asunción de sus contenidos, especialmente para la información dada por autores que no utilizaban la genitalia para la identificación de las especies, pero es obvio que mucha de esta información sí se ha tenido en cuenta, dada la autoridad de los autores que la han aportado y que merecen toda nuestra confianza.

Para la morfología general (figs. 5-33) se sigue la terminología habitualmente usada para este orden (H. Aspöck et al., 1980a, 1991). Para su identificación se aporta una clave de familias, géneros y especies, y aunque existen datos en la morfología externa que ayudan a la identificación de algunas especies, y así los utilizamos en las claves, el estudio de la genitalia es absolutamente necesario para la segura y correcta identificación de los ejemplares. Para ello se ha procedido a la separación de los últimos cuatro segmentos del abdomen mediante disección. Si el material estaba conservado en seco, se ha hidratado previamente durante 24 horas por inmersión en agua. Tanto el material así reblandecido, como el material conservado en alcohol, posteriormente se han aclarado estos últimos segmentos con hidróxido potásico al 10\% para acceder a las estructuras genitales, que en el caso de la genitalia interna de las +9 se han teñido con negro de clorazol para visualizar sus estructuras internas. Una vez realizado este proceso, se ha introducido la genitalia en glicerina en un porta excavado para su observación mediante un estereo-microscopio LEICA GZ-4. Los dibujos se realizaron utilizando el programa de diseño gráfico Corel Draw 10, indicándose, en su caso, las fuentes bibliográficas que nos han servido de modelo. Para comodidad del lector, cuando en ellos se desea resaltar un carácter, se ha utilizado en ocasiones una flecha para señalarlo.

Los datos que ahora anotamos de las especies citadas en la zona de estudio sobre su morfología, variabilidad, biología, preferencia de sustrato, ciclo biológico, fenología y amplitud altitudinal y, en su caso, sobre sus estadios larvarios conocidos y parásitos sobre ellas citados, se basan en nuestra propia información, a la que hemos añadido la aportada por autores de reconocida solvencia y la de los datos bibiográficos según los criterios anotados por Monserrat \& Triviño (2013), incluyendo, cuando proceda, un apartado final de discusión donde se comentan los datos que hemos considerado necesario anotar en cada caso.

Para la exposición del material estudiado de cada una de estas especies, los datos se exponen alfabética y cronológicamente ordenados, indicándose por este orden: el país (España y Portugal), provincia y localidad de captura, coordenadas UTM o geográficas, altitud en m (considerando $10 \mathrm{~m}$ como dato a nivel del mar), y fecha de captura, planta sobre la que los ejemplares han sido capturados, número de $\widehat{\partial} \hat{\partial} \mathrm{y}$ de $\not \rightarrow$, estudiados, recolector e institución a la que el material pertenece según las siglas anteriormente anotadas. Con la intención de no reiterar información, en la enumeración de las localidades de captura, éstas se separarán por un punto cuando correspondan a diferentes provincias, localidades $\mathrm{y} / \mathrm{o}$ fechas, y por una coma cuando todo o parte de la ubicación o nombre de la localidad ya haya sido anotada en los ejemplares citados inmediatamente antes, pormenorizándose sólo las diferencias en su fecha de captura, número de ejemplares, planta soporte, recolector o institución. Se señalan con un asterisco (*) aquellos ejemplares elegidos para realizar los dibujos de las alas $\left(a^{*}\right)$ o las figuras de la genitalia masculina $\left({ }^{*}\right)$ o femenina $\left({ }^{*}\right)$. Con el fín de reducir texto en esta relación del material estudiado se apocopa el género de la planta soporte que ya haya sido citada en cada especie. El conjunto del material estudiado, mayoritariamente inédito, asciende a 1.073 ejemplares, y en ellos basamos esta contribución.

Para la distribución general citada de cada una de las especies anotadas se sigue la recopilada por H. Aspöck et al. (1980, 1991, 2001). Los datos de distribución en la Península Ibérica que se anotan en el texto son generales, simplemente indicando si su distribución en la zona de estudio se circunscribe a zonas de influencia eurosiberiana, mediterránea, o ambas, o a determinados enclaves, y un mayor detalle sobre la distribución conocida de las especies en la zona estudiada se anota en sus mapas de distribución (figs. 156-171). Para la realización de los mapas de distribución de las especies, se ha utilizado el mismo programa de diseño gráfico anteriormente citado $\mathrm{y}$, no solo se ha tenido en cuenta el material estudiado, sino también todas aquellas citas que nos han resultado incuestionables o absolutamente solventes en función de los autores que las han generado.

\section{Resultados}

Entre las diferentes opiniones que se han vertido sobre la taxonomía y sistemática de esta familia, para la ordenación de los taxa citados se siguen los criterios utilizados por H. Aspöck et al. (1980a, 1991, 2001), salvo la consideración como especies (y no subespecies) de Ohmella baetica (Rambur, 1842) y Ohmella bolivari (Navás, 1915), siguiendo la opinión que ya manifestaron Monserrat \& Triviño (2013). 
LISTADO DE LAS ESPECIES CONSIDERADAS

Raphidiidae

Phaeostigma (Phaeostigma) notatum (Fabricius, 1781)

Phaeostigma (Phaeostigma) italogallicum (H. Aspöck

\& U. Aspöck, 1976)

Subilla aliena (Navás, 1915)

Xanthostigma aloysianum (Costa 1885)

Xanthostigma corsicum (Hagen, 1867)

Atlantoraphidia maculicollis (Stephens, 1836)

Harraphidia (Flavoraphidia) laufferi (Navás, 1915)

Harraphidia (Harraphidia) subdesertica Monserrat

\& Papenberg, 2006

Hispanoraphidia castellana (Navás, 1915)

Ohmella baetica (Rambur, 1842)

Ohmella bolivari (Navás, 1915)

Ohmella postulata (H. Aspöck \& U. Aspöck, 1977)

Ohmella libidinosa (H. Aspöck \& U. Aspöck, 1971)

Ohmella casta (H. Aspöck \& U. Aspöck, 1968)

Venustoraphidia conviventibus Monserrat \&

Papenberg, 2012

Inocelliidae

Fibla (Fibla) hesperica Navás, 1915

Claves de familias, GÉneros $\mathrm{Y}$ especies DE LA Península IbÉRICA

Aunque tratamos de utilizar para esta clave ciertos caracteres de morfología externa y venación que ayuden a la identificación a nivel de familia $y$, en cada caso, hasta nivel de especie, la genitalia es imprescindible, por lo que se anota por separado una clave para $\widehat{\jmath} \widehat{\jmath}$ y otra para +9 (en algunas especies ibéricas de algunos géneros como Venustoraphidia, se desconoce la hembra, y en otras del género Ohmella, sin adecuada diferenciación, utilizamos su distinta distribución geográfica). Para la terminología alar y genital puede seguirse la información general anotada en las figuras 16, 17, 24-42. Para la de cada género y especie se anota en las correspondientes figuras.

En esta clave se segregan los taxa a nivel de familia, de género y de especie. Para los géneros monoespecíficos (Atlantoraphidia, Subilla, Hispanoraphidia, Venustoraphidia y Fibla), alcanzado el nivel de género, se consigue la identificación a nivel de especie, según se indica. Para los restantes géneros (Phaeostigma, Xanthostigma, Harraphidia y Ohmella) se añaden otros pasos para la identificación de sus correspondientes especies.

\section{Clave de familias de Raphidioptera de la Península Ibérica:}

1 Cabeza triangular, con 3 ocelos (figs. 5, 6). El pterostigma se encuentra delimitado en su región proximal y distal por una vena, y se encuentra, en la mayoría de los casos, atravesado por una o más venas (figs. 24-38, 40, 41)

Raphidiidae

Cabeza cuadrangular, sin ocelos (figs. 7, 8). El pterostigma se encuentra delimitado solo en su región distal por una vena y nunca se encuentra atravesado por una vena o más venas (figs. 39, 42) Inocelliidae
Clave de géneros y especies de la familia Raphidiidae de la Península Ibérica:

1 Alas con el pterostigma pardo-amarillento y atravesado por una sola vena (figs. 40, 41, 26-38) .............................. 2 Alas con el pterostigma marrón oscuro y atravesado por una vena que se bifurca o por dos venas (figs. 24, 25)

Phaeostigma 9

2 Alas con vena apical (figs. 29-38, 40, 41)....................... 3 Alas sin vena apical (figs. 26-28) ........................................10

3 Proceso del gonocoxito no es largo, ni convexo con el extremo curvado en dirección dorsal (figs. 61, 66, 68, 70)

Proceso del gonocoxito es largo, convexo con el extremo curvado en dirección dorsal (figs. 64, 65)

Hispanoraphidia, Hispanoraphidia castellana

4 Estilos no bifurcados (figs. 47-56, 64-66, 68, 70, 72, 74)

Estilos bifurcados (figs. 57, 59,61)

5 Proceso del gonocoxito claramente prominente (figs. 65,

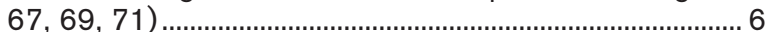

Proceso del gonocoxito no prominente (figs. 74, 75) Ohmella casta

6 Gonocoxito ancho, no prolongado en un largo proceso (figs. 66, 68, 72)......

Gonocoxito prolongado en un largo proceso en forma de pico (fig. 70) ........................................ Ohmella postulata

7 Proceso del gonocoxito orientado en dirección dorsal (figs. 66, 68)............................................................................. 8

Proceso del gonocoxito orientado en dirección caudal (fig. 72)

Ohmella libidinosa

8 Parámeros cóncavos (figs. 66, 67). Los escleritos de la hipovalva forman una curvatura en su extremo final. (fig. 67). Distribución limitada al sur de España (fig. 165)

Ohmella baetica

Parámeros rectilíneos (fig. 68). Los escleritos de la hipovalva no forman una curvatura en su extremo final (figs. 68, 69). Distribución limitada al centro occidental peninsular (fig. 166).

Ohmella bolivari

9 En la mayoría de los casos en el ala anterior aparecen 3 celdillas postigmales (fig. 25). Dos procesos del gonocoxito en forma de espina orientados en dirección dorsal (fig. 45) .................... Phaeostigma (Ph.) italogallicum En la mayoría de los casos en el ala anterior aparecen 4 celdillas postigmales (fig. 24). Un proceso del gonocoxito orientado en dirección ventral (fig. 43).

Phaeostigma (Ph.) notatum

10 Entre el Radio y el Sector del radio del ala anterior hay junto al radio 2 celdillas (figs. 26, 27)............................... 11 Entre el Radio y el Sector del radio del ala anterior hay junto al radio 3 celdillas (fig. 28 ).....

Xanthostigma corsicum

11 La parte basal de la $\mathrm{Ma}$ en el ala posterior transcurre como vena transversal (figs. 27, 29, 38) ......................... 12

La parte basal de la Ma en el ala posterior transcurre como vena longitudinal (fig. 26) ............. Subilla, Subilla aliena

12 Estilo muy largo y rectilíneo. Existe un proceso del gonocoxito (figs. 49, 50).............Xanthostigma aloysianum Estilo corto y con su extremo orientado en dirección dorsal. No existe un proceso del gonocoxito (figs. 76, 77) ..... Venustoraphidia, Venustoraphidia conviventibus

13 Gonocoxitos sin ningún proceso desarrollado de forma especial, faltan los parámeros (figs. 59,61 )...................... 14 Gonocoxitos con un proceso corto y puntiagudo, existen los parámeros (figs. 57,58 ).

Atlantoraphidia, Atlantoraphidia maculicollis

14 Ala anterior con una vena transversal suplementaria entre la Costa y el Radio (fig. 30). Hipovalva ensanchada en su zona media y con procesos laterales orientados hacia la 
línea media (figs. 59, 60).

Harraphidia (Harraphidia) subdesertica Ala anterior sin una vena transversal suplementaria entre la Costa y el Radio (fig. 31). Hipovalva levemente ensanchada en su zona media y sin procesos laterales (figs. 61, 62)...... Harraphidia (Flavoraphidia) laufferi

1 Alas con el pterostigma pardo-amarillento y atravesado por una sola vena (figs. 40, 41, 26-38). No presenta subgenitalia (figs. 103-116).

- $\quad$ Alas con el pterostigma marrón oscuro y atravesado por una vena que se bifurca o por dos venas (figs. 24, 25). Presenta subgenitalia (figs. 100-102, 118)............ Phaeostigma 11

2 Alas con vena apical (figs. 29-38, 40, 41).........................3

- Alas sin vena apical (figs. 26-28) ....................................... 12

3 El $8^{\circ}$ terguito no alcanza la zona media ventral (fig. 109) o bien alcanzando ampliamente la zona media, pero no en forma de banda, sino con una prolongación convexa orientada en dirección anterior (figs. 107, 108)................4 El $8^{\circ}$ terguito forma una banda larga y estrecha que alcanza la zona media ventral. El receptáculo seminal presenta dos procesos perpendiculares característicos (figs. 110, 133, 134).

Hispanoraphidia, Hispanoraphidia castellana

4 El ductus receptaculi es largo y fino (figs. 131, 132). El receptáculo seminal no presenta procesos (figs. 131, 132). Harraphidia 5 El ductus receptaculi es corto, fino y el receptáculo seminal no presenta procesos (fig. 161) o es largo, grueso y el receptáculo seminal presenta dos procesos (figs. 135 137-139)...

$5 \quad$ Ala anterior con una vena transversal suplementaria entre la Costa y el Radio (fig. 30). El ductus receptaculi no se encuentra enrollado en forma de ovillo, sino que está levemente festoneado (fig. 132)

Harraphidia (Harraphidia) subdesertica Ala anterior sin una vena transversal suplementaria entre la Costa y el Radio (fig. 31). El ductus receptaculi es extremadamente largo, delgado y está enrollado en forma de ovillo (fig. 131).............. Harraphidia (Flavoraphidia) laufferi

6 El atrio de la bolsa copuladora está doblado y no se encuentra esclerotizado. El sáculo es llamativamente grande y de paredes gruesas (figs. 135-139)......................... Ohmella 7 El atrio de la bolsa copuladora tiene forma de cápsula y se encuentra fuertemente esclerotizado, presentando en vista dorsal un característico dibujo en forma de herradura (fig. 130). El sáculo es poco llamativo y de paredes delicadas (figs. 129, 130)

Atlantoraphidia, Atlantoraphidia maculicollis

7 Región esclerotizada del $8^{\circ}$ terguito a la altura del espiráculo fuertemente convexa en dirección anterior (figs. 111-114)

Región esclerotizada del $8^{\circ}$ terguito a la altura del.... ráculo solo escasamente convexa en dirección anterior (figs. 116, 122)

Ohmella casta

8 El $7^{\circ}$ esternito no se encuentra muy prolongado en dirección caudal. Su borde dorsocaudal no es cóncavo (figs. $119,121)$

....9 El $7^{\circ}$ esternito se encuentra prolongado en dirección caudal (fig. 113). Su borde dorsocaudal es cóncavo (fig. 120). El ductus receptaculi presenta unas micro-espinas características en su zona media (fig. 138)......... Ohmella postulata

9 El intersegmento 7/8 sin placas esclerotizadas (figs. 111,

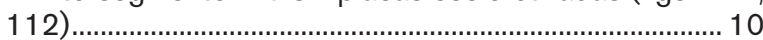
El intersegmento $7 / 8$ con dos placas esclerotizadas (fig. 115). Genitalia interna (fig. 139)..... Ohmella libidinosa

10 Distribución limitada al sur de España (fig. 165) Ohmella baetica
Distribución limitada a la región occidental del centro peninsular (fig. 166). Genitalia interna (fig. 138)

Ohmella bolivari

11 En la mayoría de los casos, en el ala anterior aparecen 3 celdillas postigmales (fig. 25). El borde caudal de la subgenitalia está fuertemente abombado (figs. 102,118) Phaeostigma (Ph.) italogallicum En la mayoría de los casos, en el ala anterior aparecen 4 celdillas postigmales (fig. 24). El borde caudal de la subgenitalia está ligeramente abombado (figs. 100, 101). Genitalia interna (figs. 124, 125) ........Phaeostigma (Ph.) notatum

12 Entre el Radio y el Sector del radio del ala anterior hay junto al radio 2 celdillas (figs. 26, 27) ............................... 13 Entre el Radio y el Sector del radio del ala anterior hay junto al radio 3 celdillas (fig. 28). Genitalia interna (fig. 128)

Xanthostigma corsicum

13 La parte basal de la Ma en el ala posterior transcurre como vena transversal. El pterostigma supera las $2 / 3$ partes de la $1^{\text {a }}$ celdilla postigmal (fig. 27). Genitalia interna (fig. 127)

Xanthostigma aloysianum

La parte basal de la $\mathrm{Ma}$ en el ala posterior transcurre como vena longitudinal. El pterostigma ocupa totalmente la $1^{a}$ celdilla postigmal (fig. 26). Genitalia interna (fig. 126)

.. Subilla, Subilla aliena

\section{Clave del género y especie de la familia Inocelliidae} de la Península Ibérica:

Único género/especie citados en la Península lbérica. Alas (figs. 39, 42), genitalia (figs. 78-80)

Fibla, Fibla (F.) hesperica

\section{오오}

Único género/especie citados en la Península lbérica. Alas (figs. 39, 42), genitalia (figs. 117, 123, 140).

Fibla, Fibla (F.) hesperica

\section{EL ORDEN RAPHIDIOPTERA EN LA PENÍNSULA IBÉRICA}

\section{Orden Raphidioptera Latreille, 1810}

Raphidia Linnaeus, 1758: Linnaeus, 1758: 552 (d). Sulzer, 1761: 45, 137-138 (mf, rf). Geoffroy, 1762: 233-234 (mf). Cederhielm, 1798: 142-143 (mf). Geoffroy, 1799 (mf). Latreille, 1802: 291 (mf). Lamarck, 1817: 199 (mf). Latreille, 1825a: 437 (mf). Burmeister, 1829: 22 (mf, tx). Burmeister, 1837: 603-607 (mf). Stein, 1838: 316, 317, 320-324 (l), 317 (h), 318-320 (mf), 325-329 (p). Burmeister, 1839: 962, 963 (gen). Erichson, 1839: 148, 159 (mf, tx). Blanchard, 1840: 64-73 (mf). Rambur, 1842: 435, 436 (mf). Schneider, 1843: 55-65 (1, mf, mon). Ratzeburg, 1844a: 228-245 (gen). Ratzeburg, 1844b (pa). Schneider, 1845: 251 (tx). Steinmann, 1963: 184 (1st)

Raphidia Linnaeus, 1758 partim: Fabricius, 1775: 314 (lst, mf). Fabricius, 1777: 110 (list, mf).

Raphidinae Latreille, 1810 partim: Latreille, 1825a: 437 (mf). Latreille, 1825b: 269 (mf).

Raphidides Latreille, 1810: Leach, 1815: 139 (lst, mf). Girard, 1879: 491-521 (gen). Albarda, 1891: 65-184 (mf mon, tx). Navás, 1911b: 35-36 (c, mf). Handlirsch, 1925: 255 (f, paleo). Handlirsch \& Beier, 1936: 1402-1413 (gen, mf). Friedrich, 1953 (gen). Kéler, 1963 (mf). Jander, 1966 (eto). Van der Ploeg, 1985 (hist)

Raphidiaedes Latreille, 1810: Billberg, 1820: 95 (1st).

Rhaphidiacea Latreille, 1810 partim: Burmeister, 1829: 22 (mf, tx). 
Raphidiina Latreille, 1810: Newman, 1834: 429 (mf). Klapálek, 1894: 489 (mf). Reuter, 1894: 29 (mf). Stitz, 1927a: 2 (a, bio, dis, mf, p). Stitz, 1927b: 49-66 (gen). Kästner, 1934: 1-11 (bio, eto). Zabel, 1941: 187-192 (bio, eto), 192, 194 (h), 192-194 (l), 195, 196 (p).

Raphidiites Latreille, 1810: Newman, 1834: 429 (mf).

Raphidiidae Latreille, 1810: Stephens, 1836: 129-132 (mf, tx). Westwood, 1840 (mf). Brauer, 1852: 72-75 (tx). McLachlan, 1868: 153, 154 (mf), 154 (a, mf), 154, 155 (p), 155 (gen). Meyer-Dür, 1875: 354 (rf). Weele, 1910: 87 (lst). EsbenPetersen, 1913a: 1-5 (gen). Comstock, 1918: 172-173 (mf). Rimski-Korsakow, 1922: 36-43 (bio, 1, p). Cockerell \& Custer, 1925: 295-297 (mf). Stitz, 1927a: 2 (tx). Lucas, 1928b: 34-41 (tx). Schimitschek, 1929 (bio, eco). Tjeder, 1937a: 143 (mf). Berland \& Grassé, 1951: 18-22 (gen, mf). Friedrich, 1953 (gen). Wichmann, 1957: 434-439 (bio, eco). Metzger, 1960a: 199-205 (bio, eco). Metzger, 1960b (gen, mf). Hoffmann, 1962: 258, 259 (gen). Achtelig, 1967: 249-312 (f, mf). Kästner, 1973 (gen).

Raphidiodea Latreille, 1810 partim: Burmeister, 1837: 603-607 (mf). Burmeister, 1839: 962-964 (mf). Boudreaux, 1979 (f). Carpenter, 1992 (paleo). Willmann, 1994: 167-195 (paleo).

Raphidea Latreille, 1810 (sic): Blanchard, 1840: 64-73 (lst)-lapsus calami!

Raphidites Latreille, 1810 partim: Blanchard, 1840: 64-73 (mf).

Raphidiodea Latreille, 1810: Schneider, 1843: 31 (mon). Tillyard, 1919: 697 (mf). Carpenter, 1936: 89-101 (mf, tx). Gustafson, 1950 (rf). Brues et al., 1954: 205, 206 (mf, paleo). Acker, 1960: 35-54 (mf). Carpenter, 1967: 270-275 (paleo). H. Aspöck \& U. Aspöck, 1968b: 53-64 (tx). H. Aspöck \& U. Aspöck, 1968c: 89-92 (tx). Adams, 1969: 9 (mf). H. Aspöck \& U. Aspöck, 1969b: 31-48 (bio, dis). Henry, 1982: 470-482 (mf).

Raphidiadea Latreille, 1810: Siebold, 1848 (mf).

Raphidiidea Latreille, 1810: Costa, 1855: 1, 2 (gen).

Sialidae Leach, 1815 partim: Brauer \& Löw, 1857: 53, 54 (mf). Brauer, 1876: 288 (1st). Esben-Petersen, 1906: 45 (rf). Ast, 1920: 417 (fis, $\mathrm{mf}$ ).

Raphidina Latreille, 1810: Wallengren, 1871: 61 (mf).

Raphidiides Latreille, 1810: Sharp, 1895: 447-448 (bio, 1, mf).

Raphidioidea Latreille, 1810: Handlirsch, 1903: 734 (f, tx). Handlirsch, 1904: 759 (f, tx). Handlirsch, 1906-1908: 38-42 (mf). Handlirsch, 1913 (paleo). Lestage, 1928: 30-32 (tx). Esben-Petersen, 1929 (mf). Tillyard, 1932: 5-13 (paleo). Klingstedt, 1937: 468-469 (cit, f). Hennig, 1953 (f, mf). Fraser, 1959: 12 (mf), 12, 13 (c). Principi, 1960: 325 (tx). Meinander, 1962: 15 (mf). Willmann, 1994: 167-195 (f, paleo).

Raphidinae Latreille, 1810: Mjöberg, 1909: 155 (dis, mf, tx).

Raphidioptera Latreille, 1810: Navás, 1916: 507 (mf), 508 (tx). Navás, 1919: 1-88 (mon). Martynov, 1925: 105-108 (mf, tx). Navás, 1928a: 5-13 (mf). Navás, 1934b: 5-10 (mf). Berland \& Grassé, 1951: 18-22 (gen). Martynova, 1960: 302-304 (f). Martynova, 1961: 71-83 (f). Berland, 1962: 17-22 (mf). Hoffmann, 1962: 258 (mf). Martynova, 1962: 270-272 (mf). Vannier \& Condé, 1962: 97 (bio), 102 (pa). Steinmann, 1963: 183-186 (tx). Metzger, 1966: 33-39 (bio). Metzger, 1967: 371-378 (f). Riek, 1967: 348 (mf). Steinmann, 1967 (mf). Günther, 1968: 399-402 (gen). Hennig, 1969 (f). U. Aspöck \& H. Aspöck, 1970: 721-723 (dis). H. Aspöck \& U. Aspöck, 1971a: 1-45 (mon). Lauterbach, 1972: 229-238 (f, mf). Meinander, 1972: 7-16 (tx). H. Aspöck, 1973: 7 (dis). Achtelig \& Kristensen, 1973: 269-272 (f). Mickoleit, 1973a: 37 (mf), 45, 46, 49, 50 (ㅇ). Mickoleit, 1973b: 145-170 (anat). Lauterbach, 1974: 1, 2 (f). U. Aspöck, 1974 (dis, mf, tx). H. Aspöck et al., 1974c: 45 (bio), 46-50 (1), 51-55 (c). Achtelig, 1974: 81-98 (pa). Achtelig, 1975b: 201-203, 235, 237 (f), 205-208, 211-217, 228-234, 236, 237 (anat). H. Aspöck et al., 1975: 368-374 (bio, eco, 1). U. Aspöck, 1975: 537-545 (dis). Kristensen, 1975: 1-44 (f). Achtelig, 1976: 233 (f, paleo). Panov \& Davydova, 1976: 187-206 (f). Achtelig, 1977: 138-163 (anat), 158-161 (bio). H. Aspöck, 1977: 14-22 (biogeo). Mamaev et al., 1977 (bio, eco). Achtelig, 1978: 140-163 ()). H. Aspöck, 1979: 243-245 (dis). Kevan, 1979: 352-354 (dis, mf). H. Aspöck et al., 1980a: 13, 437 (gen), 35, 36, 73 (c), 76 (dis), 83, 84, 382, 386-395 (mf), 330, 334 (bio), 335, 337 (biogeo). H. Aspöck et al., 1980b: 73 (bio). Büning, 1980: 127-131 (anat, f). H. Aspöck, 1981: 180, 181(dis). U. Aspöck, 1981: 171-173 (bio). Hennig, 1981 (f, mf). Kristensen, 1981: 135-157 (f). Penny, 1981: 90 (lst). Weidner, 1982 (mf). Ressl, 1983 (eco, pa). Gepp, 1984: 205, 206 (bio), 188, 189 (c), 188 (l), 204 (gen). Steinmann \& Zombori, 1984 (l, p). H. Aspöck et al., 1985a: 386-388 (pa). H. Aspöck et al., 1985b: 37, 38 (tx). Steinmann \& Zombori, 1985 (mf). H. Aspöck, 1986: 15, 16, 28 (gen), 18, 23, 24 (dis), 22 (bio). Monserrat, 1986b: 175 (lst). Paulus, 1986a: 158-160 (anat). Paulus, 1986b: 362 (anat). Schlüter, 1986: 104-105 (f). Dorokhova, 1987: 27-35 (dis, md). H. Aspöck, 1987: 148-158 (biogeo, dis). Greve, 1987: 1 (gen), 2 (mf), 35 (c), 37 (rf). Séméria \& Berland, 1988: 32-35 (gen). Büchs, 1988 (eco). H. Aspöck et al., 1989: 67-110 (biogeo, dis). H. Aspöck, 1990: 47, 48 (biogeo, dis). H. Aspöck, 1991: 26-33 (bio, eco). H. Aspöck et al., 1991: vol. I: 124, 179 (c), 180 (tx), vol. II: 406, 407 (dis). H. Aspöck \& U. Aspöck, 1991: 521-523 (mf), 523 (h, 1), 523, 524 (bio), 524 (fe, pa). U. Aspöck, 1991: 239-244 (biogeo, dis). Kristensen, 1991: 125-140 (f). Kukalová-Peck, 1991 (paleo). Oswald \& Penny, 1991: 63 (cat). H. Aspöck, 1992: 43, 46, 47 (gen). U. Aspöck et al., 1992: 170, 180-183 (biogeo), 178-189 (dis). H. Aspöck \& U. Aspöck, 1994: 338 (h), 338, 339 (a, 1, mf), 339 (bio, p, ô, ㅇ). U. Aspöck et al., 1994b: 145, 146, 148 (bio). Büning, 1994 (f, mf). Günther, 1994: 221-225 (gen). Horstmann, 1994: 79-90 (pa). Ren \& Hong, 1994: 103-108 (f). H. Aspöck et al., 1995: 181, 182 (biogeo). U. Aspöck, 1995: 633 (tx). H. Aspöck \& Hölzel, 1996: 32, 80 (rf), 33, 38, 40-45 (dis), 35 (tx). U. Aspöck \& H. Aspöck, 1996: 277, 280, 285 (gen), 278, 279, 281 (biogeo), 284 (tx). H. Aspöck, 1997: 114 (bio). Penny et al., 1997: 89-92 (cat). Wachmann \& Saure, 1997 (mf). Whiting et al., 1997: 2-40 (f). H. Aspöck, 1998a: 7-28 (hist). H. Aspöck, 1998b: 33-38 (dis), 38-39 (tx), 39-42 (paleo). U. Aspöck \& H. Aspöck, 1999a: 4, 5 (dis), 6, 11, 15 (1), 9 (gen), 7, 14 (bio), 13, 15 (h), 13, 18 (p), 18, 19, 20 (tx). U. Aspöck et al., 2001: 78, 81 (f). Haring et al., 2011: 1, 9 (tx). Monserrat \& Triviño, 2013: 15, 22 (cat, dis, gen, 1st, sin, tx).

Raphidiida Latreille, 1810: Krausse \& Wolff, 1919 (nom).

Megaloptera Latreille, 1802 partim: Killington, 1929: 2, 4 (mf).

Raphidioidea Latreille, 1810 partim: Tjeder, 1937b: 118-124 (mf).

Raphidioptera Latreille, 1810 partim: Kästner, 1973 (gen). Seifert, 1975 (1st).

Raphidoidea Latreille, 1810: Matsuda, 1976 (mf).

Raphidida Latreille, 1810: Boudreaux, 1979 (f).

Rhaphidioptera Latreille, 1810: Dettner \& Peters, 1999 (gen).

Familia Raphidiidae Latreille, 1810, sensu Navás, 1916

Raphidia Linnaeus, 1758: Linnaeus, 1758: 552 (d). Latreille, 1796 (gen). Latreille, 1802: 291 (gen). Latreille, 1805 (rf). Latreille, 1807 (gen). Goldfuss, 1820 (gen). Latreille, 1825b: 268 (mf). Burmeister 1829: 22 (tx). Stephens, 1836: 129-132 (gen, tx). Burmeister, 1837: 603-607 (gen). Schneider, 1843: 55-66 (1, mf, mon, p, tx). Hagen, 1844: 180 (rf). Guérin-Méneville, 1845: 34 (bio, p). Erichson, 1846 (bio). Loew, 1848 (anat). 
Siebold, 1848 (mf). Brauer, 1852: 72-75 (tx). Brauer, 1855b: 483 (1), 723 (mf). Costa, 1855: 1 (gen). Brauer \& Löw 1857: 53 (mf). Douglas 1859: 69 (bio). Wallengren, 1871: 63 (a). Brauer, 1876: 288 (lst). Girard, 1879: 491-521 (gen). Redtenbacher, 1886 (mf). Albarda, 1891: 82 (rf), 85 (ぶ), 85-90 (a). Brongniart, 1893 (mf). Sharp, 1895: 447-448 (bio, 1, mf). Stitz, 1909: 379 (す). Mjöberg, 1909: 156 (dis, mf). Navás, 1911b: 35-36 (c, mf). Müller, 1912: 219-240 (mf). Esben-Petersen, 1913a: 6 (a, mf). Navás, 1915h (mf). Comstock, 1918: 172-173 (mf). Stitz, 1927a: 2 (c). Mac Gillavry, 1949: 116-122 (gen). Aubert, 1961: 195-200 (pa). Achtelig, 1967: 249-312 (anat, f, mf).

Raphidies Latreille, 1810: De Geer, 1779 (mf).

Raphidinae Latreille, 1810: Latreille, 1810 (d) [género tipo: Raphidia Linnaeus, 1758].

Raphidini Latreille, 1810 sensu Navás, 1913: Navás 1913b: 11 (tx) [género tipo: Raphidia Linnaeus, 1758].

Raphidiidae Latreille, 1810 sensu Navás, 1916: Navás, 1916: 508, 509 (mf). Navás, 1919: 3 (rf), 6 (dis), 11 (c). Crampton, 1918: 52 (mf). Withycombe, 1923: 33 (rf). Lestage, 1928: 32 (tx). Lucas, 1928b: 34-41 (bio). Navás, 1928a: 13 (mf). Killington, 1929: 5 (mf). Schimitschek, 1931 (bio). Navás, 1934b: 11 (c), 12 (mf). Carpenter, 1936: 93-96 (tx), 97, 98 (a), 101-104 (mf), 105 (h), 105, 106 (l), 107 (p). Naville \& de Beamont, 1936 (1st). Ferris \& Pennebaker, 1939: 121-142 (mf). Lakon, 1939: 517-526 (pa). Weber, 1939: 522-535 (pa, patol). Clausen, 1940 (bio). Hinton, 1947: 288 (p). Mac Gillavry, 1949: 116-122 (hist). Thompson, 1950 (pa). Czihak, 1952: 1-70 (anat, bio, mf). Principi, 1952: 2 (tx). Hennig, 1953 (f, mf). Brues et al., 1954: 205 (mf). Tjeder, 1954: 26 (mf). Matsuda, 1956a: 146-155 (anat, mf). Matsuda, 1956b: 1-65 (anat, mf). Perkins, 1956: 159-160 (pa). Matsuda, 1957: 39-63 (anat, mf). Snodgrass, 1957 (mf). Henry, 1958: 95-105 (anat). Metzger, 1958 (bio, mf). Schwenke, 1958 (eco). Woglum \& McGregor, 1958: 129-141 (bio). Woglum \& McGregor, 1959: 489-502 (bio). Acker, 1960: 35-47, 53 (mf). Peterson, 1960: 352-361 (1). Principi, 1960: 332 (mf). Martynova, 1961: 71-83 (f). Martynova, 1962: 270-272 (mf). Meinander, 1962: 15 (c), 15, 16 (mf). Steinmann, 1963: 183-186 (tx). H. Aspöck \& U. Aspöck, 1964: 136, 137 (mf). Mickoleit, 1965: 521-531 (anat). Acker, 1966: 1-6 (eto). Jander, 1966 (eto). Steinmann, 1967 (mf). H. Aspöck \& U. Aspöck, 1968b: 53-64 (tx). Adams, 1969: 9 (mf). Metzger, 1969: 897-909 (mf). Mickoleit, 1969 (anat). Rohdendorf, 1969 (paleo). Tjeder, 1970: 89 (mf). Tuxen, 1970 (mf). H. Aspöck \& U. Aspöck, 1971a: 5 (tx), 6 (c). Lauterbach, 1972: 230, 232, 233, 237 (mf), 239, 243-247 (ठ). Horstmann, 1973: 1-14 (pa). Popov, 1973: 121 (dis, tx). H. Aspöck et al., 1974a: 45 (rf), 51 (1). U. Aspöck, 1974 (dis, tx). Lauterbach, 1974: 1 (f). Wyniger, 1974 (t). Achtelig, 1975b: 205 (anat), 207 (rf). H. Aspöck et al., 1975: 371 (bio). U. Aspöck, 1975: 538-540 (dis). Matsuda, 1976 (mf). Panov \& Davydova, 1976: 187-206 (fis). Achtelig, 1977: 139, 141 (mf), 143, 156, 157 (ㅇ). Mamaev et al., 1977 (bio, eco, 1). Yang, 1978 (gen). H. Aspöck et al., 1980a: 21, 345 (gen), 36, 73 (c), 76, 86, 337-339, 343, 344 (dis), 84, 85, 127 (tx), 85, 381, 386 (a), 85, 389, 391 (ठ), 85, 390, 394 (우), 329, 331 (bio), 332 (pa), 334 (rf). Büning, 1980: 127-131 (anat, f). Hennig, 1981 (f). Henry, 1982: 470-482 (gen). Gepp, 1984: 189 (mf), 189, 205 (1), 204 (gen). H. Aspöck et al., 1985b: 38 (tx). H. Aspöck, 1987: 148, 149, 152 (gen). Greve, 1987: 35 (c). Tauber, 1987: 124 (c, gen). H. Aspöck et al., 1989: 67 (tx), 70, 74-84, 87-109 (biogeo). H. Aspöck, 1990: 47 (tx), 48, 54, 55 (biogeo), 58 (f). H. Aspöck, 1991: 27 (tx), 28 (1). H. Aspöck \& U. Aspöck, 1991: 521 (gen, mf), 523 (l), 524 (fe). H. Aspöck et al., 1991: vol. I: 124-172 (c), 183 (lst), vol. II: 408, 409 (dis). U. Aspöck, 1991: 239-244 (biogeo, dis). U. Aspöck \& H. Aspöck, 1991:
25, 31 (dis). Oswald \& Penny, 1991: 63 (cat). H. Aspöck, 1992: 45, 46 (dis). U. Aspöck et al., 1994a: 393-395, 398-401 (bio), 401 (ð). U. Aspöck et al., 1994b: 146, 148 (bio), 148 (eto). Günther, 1994: 221-225 (gen). Ren \& Hong, 1994: 103-108 (f). Willmann, 1994: 167-197 (f). H. Aspöck et al., 1995: 167182 (dis, mf, tx). H. Aspöck et al., 1996: 195-214 (dis, mf, tx). U. Aspöck \& H. Aspöck, 1996: 227, 279 (gen), 279 (lst). H. Aspöck \& Hölzel, 1996: 38-45 (cat, dis). H. Aspöck et al., 1997: 78-94 (dis, mf, tx). H. Aspöck, 1997: 114 (bio). Penny et al., 1997: 89-92 (cat). Wachmann \& Saure, 1997 (mf). H. Aspöck, 1998a: 13, 14 (hist). H. Aspöck, 1998b: 33, 34 (gen), 34 (1, fe, p), 34, 35 (dis), 36, 38, 39 (f). H. Aspöck et al., 1998b: 115 (gen), 115, 116 (lst, rf), 123 (biogeo), 124-127 (bio). U. Aspöck \& H. Aspöck, 1999a: 4 (dis), 5, 6 (l), 7 (bio), 8, 9 (mf). H. Aspöck \& U. Aspöck, 2007: 100 (tx). Haring et al., 2011: 1 (gen), 2 (dis). H. Aspöck \& U. Aspöck, 2012: 217 (gen), 218 (bio, h, p), 218, 219 (dis), 219 (tx). Monserrat \& Triviño, 2013: 15,22 (cat, dis, gen, 1st, sin, tx).

Raphidini Latreille, 1810 sensu Navás, 1918 partim: Navás, 1918a: 22 (mf). Navás, 1919: 81 (tx). Lestage, 1928: 30 (rf). Navás, 1928a: 14 (mf). Navás, 1934b: 12 (mf). Carpenter, 1936: 92 (nom, rf). H. Aspöck \& U. Aspöck, 1971a: 5 (rf). H. Aspöck \& U. Aspöck, 1971e: 108 (rf).

Ermini Navás, 1918 partim: Navás, 1918a (d) [género tipo: Erma Navás, 1918a]. Navás, 1919: 81 (mf). Navás, 1928a: 40 (mf). Navás, 1934b: 47 (mf). Carpenter, 1936: 92 (nom, rf). H. Aspöck \& U. Aspöck, 1971a: 5 (rf). H. Aspöck \& U. Aspöck, 1971e: 108 (rf).

Raphidiinae Latreille, 1810 sensu Lestage, 1928 partim: Lestage 1928: 32 (tx). Carpenter, 1936: 95 (nom, rf). H. Aspöck \& U. Aspöck, 1971a: 5 (rf).

Raphidillinae Lestage, 1928 partim: Lestage, 1928: 32 (d) [género tipo: Raphidilla Navás, 1915d]. Carpenter, 1936: 95 (nom, rf). H. Aspöck \& U. Aspöck, 1971a: 5 (rf).

Ermidae Navás, 1918 partim: Lestage, 1928: 32 (nom, tx). Carpenter 1936: 95 (nom, rf). Steinmann, 1963: 184 (1st). H. Aspöck \& U. Aspöck, 1971a: 5 (rf). H. Aspöck \& U. Aspöck, 1971e: 108 (rf).

Raphidiinae Latreille sensu Handlirsch \& Beier, 1936: Handlirsch \& Beier, 1936: 1402-1413 (gen, mf). Berland \& Grassé, 1951: 18-22 (mf). Hoffmann, 1962: 259 (rf).

Raphidiinae Latreille sensu Raphidini Navás, 1918 partim: Friedrich, 1953 (tx).

Erminae, Navás 1918 partim: Friedrich, 1953 (tx). H. Aspöck \& U. Aspöck, 1971e: 108 (rf).

\section{Género Phaeostigma Navás, 1909}

Phaeostigma Navás, 1909b: 1 (d), [especie tipo por designación posterior: Raphidia notata Fabricius, 1781].

Considerado como sección de Raphidia Linnaeus, 1758: Navás, 1909b: 1 (a, d).

Considerado como subgénero de Raphidia Linnaeus, 1758: H. Aspöck et al., 1975: 372 (eco, 1). H. Aspöck \& U. Aspöck, 1976: 59-69 (dis, mf, tx). H. Aspöck, 1977: 19 (dis). H. Aspöck, 1979: 249, 253 (dis). H. Aspöck et al., 1980a: vol. I: 86 (lst), 87, 97 (dis), 96, 97 (tx), 97 (bio), 100, 106-108 (rf), vol. II: 237 (dis).

Considerado como género: H. Aspöck \& U. Aspöck, 1973a: 52 (nom). H. Aspöck, 1986: 23 (tx), 25 (dis). H. Aspöck, 1987: 156 (dis). H. Aspöck et al., 1989: 90, 95 (dis). H. Aspöck et al., 1991: vol. I: 76 (fe), 77, 105, 109, 112, 620 (rf), 111, 113, 184, 185, 187 (tx), 114, 186, 187, 558 (dis), 185 (a, mf, ふ̂, ㅇ), 186 (bio, 1), vol. II: 410 (dis). Oswald \& Penny, 1991: 65 (1st). H. Aspöck \& Hölzel, 1996: 38 (dis), 39 (gen). H. Aspöck et al., 2001: 19, 20 (tx), 20 (dis). H. Aspöck, 2002: 44, 45 (pa, 1). H. Aspöck \& U. Aspöck, 2007: 97 (dis), 100 (rf). Monserrat \& Papenberg, 2006: 204 (rf). Monserrat \& Papenberg, 2010: 48 (rf, tx), 48, 52 (dis), 
50 (a, fe, ô,, , mf, 1), 50, 52 (bio). Haring et al., 2011: 11-13 (tx), fig.S5 (dis). U. Aspöck et al., 2012: 576, 579 (f), 577 (lst), 580 (tx). Monserrat \& Papenberg, 2012: 292 (rf). H. Aspöck \& U. Aspöck, 2013: 12 (tx), 41, 42 (gen).

Phaeostigma es un género bien delimitado, formado por nueve subgéneros con 42 especies, más 2 especies incertae sedis. Es el género de la familia Raphidiidae con el mayor número de especies. Citado en Europa, Chipre, Turquía, región del Caúcaso, Líbano, Siria, norte de Irán, norte de Irak. En la Península Ibérica hasta la actualidad solo están citadas las especies Phaeostigma (Phaeostigma) notatum y Phaeostigma (Phaeostigma) italogallicum, ambas pertenecientes al subgénero Phaeostigma.

\section{Subgénero Phaeostigma Navás, 1909}

Phaeostigma Navás, 1909b: 1 (d), [especie tipo por designación posterior: Raphidia notata Fabricius, 1781].

Considerado como subgénero de Raphidia Linnaeus, 1758: H. Aspöck et al., 1975: 372 (eco, 1). H. Aspöck \& U. Aspöck, 1976: 59-69 (dis, mf). H. Aspöck, 1977: 19 (dis). H. Aspöck, 1979: 249, 253 (dis). H. Aspöck et al., 1980a: vol. I: 86 (lst), 87, 97 (dis), 96, 97 (tx), 97 (bio), 100, 106-108 (rf), vol. II: 237 (dis).

Considerado como subgénero de Phaeostigma Navás, 1909: H. Aspöck et al., 1983a: 114, 115, 118 (dis), 117 (tx). H. Aspöck et al., 1989: 90, 95 (dis). H. Aspöck et al., 1991:vol. I: 111, 185, 187, 188 (pa, tx), 187 (a, mf), 187, 188 (ふ) 188 (bio, 1, 우), 188, 558 (dis), vol. II: 410 (dis). H. Aspöck \& Hölzel, 1996: 39 (dis). H. Aspöck et al., 2001: 20 (dis, tx). H. Aspöck, 2002: 37 (1). Letardi \& Pantaleoni, 2007: 89 (lst). Monserrat \& Papenberg, 2010: 48, 54 (tx), 52 (a, l, mf), 52, 54 (ふ) 54 bio, dis, fe, ㅇ).

= Erma Navás, 1918a: 20 (d, a, mf), [especie tipo por designación original: Erma abdita Navás, 1918].

Navás, 1919: 81, 82 (mf), 82 (a). Lestage, 1928: 32 (c). Navás, 1928a: 40, 41 (mf), 41 (a). Navás, 1933a: 121 (lst). Navás, 1934a: 6 (1st). Navás, 1934b: 47 (mf), 47, 48 (a). Carpenter, 1936: 100 (dis). Berland \& Grassé, 1951: 18 (rf). Friedrich, 1953 (lst). Metzger, 1960b (rf). Steinmann, 1963: 184 (lst). H. Aspöck \& U. Aspöck, 1971a: 5 (rf). H. Aspöck \& U. Aspöck, 1971e: 108 (rf). H. Aspöck \& U. Aspöck, 1976: 59 (sin). H. Aspöck et al., 1991: 105, 106 (tx), 187 (lst). Oswald \& Penny, 1991: 65 (lst). Monserrat \& Papenberg, 2010: 48 (tx).

= Navasana Steinmann, 1963: 186 (d), [especie tipo por designación original: Navasana perumbrata Steinmann, 1963].

Steinmann, 1967 (tx). Buresch \& Popov, 1973: 155 (rf). H. Aspöck et al., 1991: vol. I: 187 (a), 111, 185, 187, 188 (dis, tx), 188 (bio, l, pa, ô, o +), 558 (dis), 187 (mf), vol. II: 410 (dis). Oswald \& Penny, 1991: 65 (1st).

Considerado como subgénero de Raphidia Linnaeus, 1758: H. Aspöck \& U. Aspöck, 1968b: 62 (tx). H. Aspöck et al., 1969: 134 (tx). H. Aspöck \& U. Aspöck, 1971e: 108 (rf), 109, 110 (ふ), 110 (dis), 111 (ㅇ). H. Aspöck \& U. Aspöck, 1972b: 25 (rf). H. Aspöck \& U. Aspöck, 1973a: 52 (sin).

Phaeostigma (Phaeostigma) notatum (Fabricius, 1781) Raphidia notata Fabricius, 1781: Fabricius, 1781 (d). Fabricius, 1787 (1st). Linnaeus, 1789: 68 (dis, mf). Linnaeus, 1790: 2648 (dis, mf). Fabricius, 1793: 99 (dis, mf, 1st, p). Billberg, 1820: 95 (mf, 1st). Latreille, 1825b: 269 (mf). Schummel, 1832 (mf). Percheron, 1833 (dis, mf). Curtis, 1837 (lst). Stein, 1837 (tx). Stein, 1838: 321 (l). Burmeister, 1839: 964 (mf). Zetterstedt, 1840: 1055 (rf). Rambur, 1842: 436, 437 (mf), 437 (a, dis). Schneider, 1843: 80 (mf, mon, tx). Ratzeburg, 1844b (rf).
Hagen, 1844: 182 (dis), 184 (rf), 185, 186 (a). Schneider, 1845: 252 (tx), 252, 253 (a, mf). Walker, 1853: 209 (a, mf). Hagen, 1854: 90 (rf). Brauer, 1855a: 723 (dis). Brauer \& Löw, 1857: 54 (c), 71 (lst). Wallengren, 1863: 16 (a), 17 (rf). Girard, 1864 675 (rf). Dziedzielewicz, 1867: 164 (lst). Hagen, 1867: 495, 496 (a), 496 (ふ), 496, 498 (ㅇ). McLachlan, 1868: 155, 156 (mf), 156, 157 (a), 220 (tx). McLachlan, 1870: 18 (lst). Wallengren, 1871: 61, 62 (a), 62 (dis, 1, mf). Rostock, 1873 (lst). Meyer-Dür, 1875: 355 (c), 357 (a, dis, mf), 430 (lst). Brauer, 1876: 266, 277, 288 (dis). Brauer, 1878: 74 (lst) Girard, 1879: 491-521 (lst). Parfitt, 1879: 403 (rf). Rostock, 1881: 224 (dis). Majewski, 1882: 15 (dis). McLachlan, 1884: 16 (dis). Majewski, 1885: 12 (dis). Schneider, 1885: 24 (dis). Schoch, 1885: 93 (1st). Brauer, 1886: 25 (bio). Schøyen, 1887 (dis). Rostock, 1888 (mf). Selys-Longchamps, 1888: 160 (dis). Albarda, 1889 (dis, sin). Albarda, 1891: 70, 74, 80, 93, 94 (a), 77, 94, 95 (dis), 70, 92, 93 (mf), 73, 75, 76, 77, 81, 82 (rf). Dziedzielewicz, 1891: 93 (rf). Martin, 1893: 142 (bio). McLachlan, 1894: 186 (dis, 1). Reuter, 1894: 12 (dis), 30 (c). Dziedzielewicz, 1895: 32 (rf). Klapálek, 1895 (lst). Morley \& Elliott, 1895: 193, 194 (fe). Heyden, 1896: 111 (bio). McLachlan, 1896: 42 (dis). Dubois, 1899: 52 (dis). Lucas, 1902: 184 (dis). Wüstnei, 1902: 135 (dis). Klapálek, 1903a: 262 (dis). Klapálek, 1903b: 5 (ठ̋). Shaw, 1903: 94 (lst). Dziedzielewicz, 1905: 112 (dis). Briggs, 1906: 363 (rf). EsbenPetersen, 1906: 45 (c, mf), 45, 46 (a), 46 (bio, dis, 1, p). Klapálek, 1906: 7 (dis). Strobl, 1906: 247 (dis). Weele, 1907 (dis). Lyle, 1908 (h). Mjöberg, 1909: 156 (dis, mf). Morley, 1909: 142 (dis), 143 (fe, mf). Stitz, 1909: 397, 405-409, 445 (ठ), 405 (mf), 429-432 (ㅇ). Navás, 1909b: 1 (dis). Navás, 1909c: 450 (dis). Porritt, 1909: 183 (dis, 1st). Rohwer, 1909 (tx). Weele, 1910: 87, 88 (1st). Lucas, 1911 (lst). Navás, 1911b: 36 (c, mf). Cockerell, 1912 (tx). Lacroix, 1912: 164 (rf). Schirmer, 1912: 140 (dis). Szilady, 1912: 57 (1st). EsbenPetersen, 1913a: 8 (dis, 1st), 15 (mf). Lacroix, 1913: 20 (c). Lyle, 1913: 187, 188 (h), 188 (bio, fe). Navás, $1913 \mathrm{c}: 107$ (lst). Williams, 1913: 7 (h). Esben-Petersen, 1914: 135 (a, c, dis, mf). Lucas, 1914a (dis). Lucas, 1914b (1st). Carr, 1914 (dis). Campion, 1915: 24 (bio, rf), 24, 25 (l), 25, 26 (fe). Navás, 1915e: 26 (tx). Wanach, 1915: 324 (a). Mac Gillavry, 1916 (dis). Lucas, 1917: 87 (1, p, rf). Mocsary, 1918: 41 (dis). Pongrácz, 1919: 169 (dis). Dziedzielewicz, 1920: 46 (dis). Haines, 1922: 84 (dis). Hellen, 1922: 25 (dis). Lackschewitz, 1922: 10 (1st), 19 (a, dis), 20 (rf). Lucas, 1922: 287 (rf). Puschnig, 1922: 75 (dis). Banks, 1923 (a). Lucas, 1923: 219 (bio, dis, pa), 219, 220 (1). Puschnig, 1923 (dis). Lucas, 1925: 155 (dis). Lundblad, 1925: 98 (ð)), 99 (mf). Killington, 1926: 110 (dis). Lucas, 1926: 290 (dis). Killington, 1927: 40 (dis, 1, p). Lucas, 1927a: 169 (dis). Lucas, 1927b: 7 (1st). Stitz, 1927a: 3 (c). Killington, 1928: 49 (dis). Lucas, 1928a: 217 (dis). Lucas, 1928b: 34-41 (bio, dis, h, tx). Tjeder, 1928: 162 (tx). Crampton, 1929: 496 (ㅇ). Killington, 1929 (c, dis, mf, p). Lackschewitz, 1929: 14 (dis). Lucas, 1929: 156 (dis). EsbenPetersen, 1929 (dis, mf). Schimitschek, 1929 (eco, mf). Lucas, 1930: 269 (dis). Titschack, 1930: 115 (dis). Killington, 1931: 73 (bio, fe, 1, p). Schimitschek, 1931 (bio). Killington, 1932a: 8 (dis). Killington, 1932b: 59 (bio), 61 (fe), 62 (pa), 64 (dis). Kästner, 1934: 1-11 (bio, eto, mf, p). Kimmins, 1934: 618 (1st). Syms, 1934: 121 (rf), 124 (bio, dis, h, 1). Klingstedt, 1935: 14 (lst). Mosely, 1935: 208 (dis). Carpenter, 1936: 104-106 (rf), 106 (1), 107 (p), 117 (ठ)). Karl, 1937: 56 (dis). Tjeder, 1937a: 143 (9, rf). Tjeder, 1937b: 120 (dis). Tjeder, 1938: 4 (dis). Zacwilichowski, 1938: 242 (dis). Alfken, 1939: 517 (dis, fe). Eglin, 1939: 164 (bio, dis), 165, 166 (h), 165, 168, 169-173, 176 (l), 168, 180 (mf), 177-180 (p), 179, 180 (a), 181-184 (fe). 
Esben-Petersen, 1939: 58 (rf). Kimmins, 1939: 12 (dis). Zacwilichowski, 1939: 9 (1st). Clausen, 1940 (bio). Eglin, 1940: 248, 266, 267 (dis), 250 (rf), 313, 342 (bio). EsbenPetersen, 1940: 1-8 (bio, dis). Tjeder, 1940a: 78, 85 (lst), 98 (a, tx). Tjeder, 1940b: 118, 119 (dis). Eglin, 1941: 71 (bio, dis). Tjeder, 1941 (dis). Zabel, 1941: 187 (rf, ie), 188, 191 (bio), 189 (O), 192 (h), 192, 193 (1). Jahn, 1942 (eco). Silvestri, 1943: 7 (mf). Tjeder, 1943a: 137 (dis). Tjeder, 1943b: 7 (dis). Kloet \& Hincks, 1945: 80 (lst). Klefbeck \& Tjeder, 1946: 205 (rf). Hinton, 1947: 288 (p). Weiss, 1947 (dis). Browning, 1948: 163 (rf). Thompson, 1950 (pa). Berland \& Grassé, 1951: 18-22 (mf). Blair, 1951: 160 (rf). Fraser, 1951: 226 (bio), 226, 227 (eco). Bartoš, 1952 (dis). Genay, 1953: 2 (dis), 22, 23 (1). Hennig, 1953 (mf). Sankey, 1953: 284 (lst). Tjeder, 1954: 26, $27\left({ }^{\Uparrow}\right)$. Condé \& Pagès, 1956a: 125 (rf). Condé \& Pagès, 1956b: 1 (dis, rf). Wichmann, 1957: 433 (bio). Carpenter, 1958: 54, $56($ đ). Metzger, 1958 (tx). Fraser, 1959: 4 (mf), 11 (a), 13 (c). Kis, 1959: 334 (dis). Metzger, 1960a: 199 (dis), 199, 200 mf). Metzger, 1960b (bio, mf, tx). Ohm, 1961a: 12 (dis). Ohm, 1961b: 2 (a). Hoffmann, 1962: 261 (c), 262 (mf), 263, 264 (a), 264 (bio). Meinander, 1962: 16 (bio, c, mf), 16, 17 (a). Remm, 1962 (dis). Meinander, 1963: 160 (dis). Meinander \& Brander, 1963: 83 (dis). H. Aspöck \& U. Aspöck, 1964: 137, 138 (bio), 137, 140 (dis), 137 (gen), 217 (c), 253, 254 (mf) 254 (ふ) ), 283, 253 (a). Bartoš, 1964: 320 (dis). Hölzel, 1964: 105 (c), 106 (a, mf), 106, 107 (dis), 153 (lst). Kis \& Stamp, 1964: 56 (dis). Kloet \& Hincks, 1964: 100 (lst). Ohm, 1964: 125 (dis). Popov, 1964: 70 (). Ressl, 1964: 64 (dis). H. Aspöck \& U. Aspöck, 1965: 344 (dis), 345 (). Bartoš, 1965a: 93 (a). Bartoš, 1965c: 136, 137 (a). Bartoš, 1965d: 228 (rf). Mickoleit, 1965: 530 (bio). Nagler, 1965 (lst). Acker, 1966: 5 (bio). H. Aspöck \& U. Aspöck, 1966a: 44 (dis). Mairhuber, 1966: 64 (dis). Metzger, 1966: 35 (bio, fe, rf), 36, 39 dis). Fischer, 1966 (dis). Jander, 1966 (eto). Bartoš, 1967: 328, 341 (rf), 330, 335, 337, 344 (a), 341 (dis), 342 (gen). Eglin-Dederding, 1968 (eco). Ohm \& Remane, 1968: 212 (dis). H. Aspöck \& U. Aspöck, 1969a: 36 (dis). Hennig, 1969 (mf). Ressl, 1969: 121-123 (bio, dis). Zelený, 1969: 15 (a). Krause \& Ohm, 1970: 26 (dis). Tjeder, 1970: 90 (ふ̋). Zelený, 1971: 39 (dis). Kleinsteuber, 1972: 64 (dis). Lauterbach, 1972: 236 (rf), 246 (ठ). Achtelig \& Kristensen, 1973: 270 (1). H. Aspöck \& U. Aspöck, 1973a: 52 (tx). Gepp, 1973: 38 (bio, gen). Hölzel, 1973: 498 (dis). Horstmann, 1973: 1-14 (pa). Joost, 1973: 146 (dis). Achtelig, 1974: 80, 81, 94 (1, pa). Gepp, 1974: 140 (tx), 143 (dis), 153 (bio). Lauterbach, 1974: 6 (ठ). Achtelig, 1975b: 205 (rf), 206, 211, 212, 216, 217, 231 (mf). Gepp, 1975: 266 (dis). Horstmann, 1975: 251-265 (pa). Horstmann, 1976: 22-31 (pa). H. Aspöck \& U. Aspöck, 1976: 59 (tx). Achtelig, 1977: 139, 144, 149-151, 152 (mf). Kofler, 1977: 338 (1), 338, 339 (dis), 339 (p). Mamaev et al., 1977 (eco, 1). Zelený, 1977: 127 (dis). Achtelig, 1978: 141 (anat), 146-148, 150, 152 (ㅇ). Kowalska, 1978: 212 (mf), 213 (rf). Ujhelyi, 1979: 87 (dis). Craik, 1980: 243 (rf). Devetak, 1980: 2 (1st). Eglin-Dederding, 1980c (eco, dis). Verdcourt, 1980: 40 (dis). Gepp, 1981 (1st). Hennig, 1981 (mf). Chládek \& Laštůvka, 1982 (eco, dis). Czechowska, 1982: 182 (dis). Ressl, 1983 (eco, dis, pa). Eglin-Dederding, 1984: 55 (bio). Devetak, 1984b: 69 (1st). Gepp, 1984: 193, 205 (1), 205 (p). Czechowska, 1985: 393, 394, 398 (eco), 394, 397 (dis). Barnard et al., 1986: 1323, 1325 (fe, 1), 1324 (bio). Czechowska, 1986: 206 (dis). Gepp, 1986b: 7 (mf). Dorokhova, 1987: 28, 30 (dis, mf, ठૈ). Greve, 1987: 35 (c), 36 (dis). Barnard, 1988: 4 (c), 4, 5 (a), 6 (mf), 7 (ô), 8 (dis, gen), 11 (1st). Starý et al., 1988: 29 (bio, eco). Dobosz, 1989: 76 (dis). Czechowska, 1990: 98, 99 (dis), 109 (gen), 101, 107 (bio). Dobosz, 1990: 63 (rf). Starý et al., 1990 (eco). Suntrup, 1990 (eco, dis, pa). Ábrahám, 1991: 16 (dis). Czechowska \& Dobosz, 1991: 139 (dis). Kovarik et al., 1991: 360 (bio). Schmitz, 1992 (dis). Sziráki et al., 1992: 114 (dis). Czechowska, 1994: 461 (rf), 462 (fe), 464, 465 (bio). Dobosz, 1994: 23 (dis). Kacirek, 1994: 70 (dis). Czechowska, 1997 (eco, dis). Monserrat \& Triviño, 2013: 15 (lst).

Raphidia (Phaeostigma) notata Fabricius, 1781: Navás, 1909b: 1 (dis). H. Aspöck \& U. Aspöck, 1973a: 53 (tx). H. Aspöck \& U. Aspöck, 1974a: 97 (a). H. Aspöck et al., 1974a: 46 (rf), 48 (bio), 48, 50, 57 (l), 53 (c). H. Aspöck et al., 1974c: 2 (rf), 3 (dis), 5-7 (l), 8, 9, 26 (pa), 9 (fe), 18 (bio), 19 (gen). Kleinsteuber, 1974: 147 (lst). Plewka, 1974: 287 (rf). H. Aspöck \& U. Aspöck, 1976: 57 (bio), 57, 69 (gen), 58, 59 (tx), 58, 60, 67-69 (dis), 59, 60, $64\left({ }^{\Uparrow}\right), 60,61,64$ (9). H. Aspöck, 1977: 17-19 (dis). Gepp, 1978: 245, 246 (dis), 246, 248 (bio), 247, 248 (fe). EglinDederding, 1979: 494 (dis). H. Aspöck et al., 1980a: vol. I: 42, 43 (c), 97 (a, bio, 1, tx), 97, 349 (dis), 340 (rf), vol. II: 25 (ふ̋), 42 (9), 160 (a), 237 (dis). Eglin-Dederding, 1980a: 304, 305, 335 (dis), 304, 335, 340, 345 (bio), 304, 336 (fe, gen, 1). EglinDederding, 1980b: 77 (bio, dis), 83 (fe). Hölzel et al., 1980: 3 (1, 1st). Malicky et al., 1983: 107 (eco). Devetak, 1984a: 56, 57 (dis). Kis, 1984 (dis, tx). Eglin-Dederding, 1986: 172 (lst), 175 (fe), 175, 188 (dis), 175, 192, 195 (bio), 175, 193 (1). Gepp, 1986a: 138 (1). Pantaleoni, 1986: 168 (rf). Büchs, 1988 (eco, dis). Séméria \& Berland, 1988: 36 (mf). Stelzl, 1989: 13 (fe), 13, 91, 119 (bio). Dobosz, 1991a: 195, 197-199 (dis), 198 (bio, 1). Dobosz, 1991b: 223 (dis). Tröger, 1993: 37 (dis). Monserrat \& Triviño, 2013: 15 (lst).

Raphidia notata var. aperta Navás, 1911: Navás, 1911a: 246 (d). Monserrat \& Triviño, 2013: 15 (lst).

Lesna notata (Fabricius, 1781): Navás, 1915a: 785, 796 (dis, 1st). Navás, 1915b: 871 (dis). Navás, 1915g: 332 (a, dis). Navás, 1915i: 542 (rf), 543 (dis). Navás, 1919: 13 (c). Navás, 1925: 43 (c). Lestage, 1928: 30 (rf). Navás, 1928c: 106 (dis). Navás, 1928d: 64 (dis). Gadeau de Kerville, 1933: 364 (dis). Lacroix, 1933: 152 (dis). Navás, 1933a: 120 (1st). Navás, 1934a: 4 (dis). Navás, 1934b: 13 (c), 13, 14 (mf), 14 (a, dis, ô, 우). Steinmann, 1963: 196 (a), 197 (c). Steinmann, 1967 (rf). H. Aspöck \& U. Aspöck, 1976: 59 (tx). Monserrat \& Triviño, 2013: 15 (lst).

Raphidia (Lesna) notata Fabricius, 1781: Condé \& Pagès, 1954: 143 (1st). Condé \& Pagès, 1956a: 127 (dis). Condé \& Pagès, 1959: 192 (dis), 193 (gen). Berland, 1962: 16, 22 (mf, rf). Vannier \& Condé, 1962: 96 (dis), 97, 99-102 (l), 98 (rf), 102 (pa). Monserrat \& Triviño, 2013: 15 (lst).

Raphidia (Navasana) notata Fabricius, 1781: H. Aspöck \& U. Aspöck, 1968b: 62 (tx). H. Aspöck et al., 1969: 133, 135 (ふ), 135 (dis). H. Aspöck \& U. Aspöck, 1971a: 37 (mf), 38 (ð), 39 ()), 40, 41 (gen), 42 (h), 44 (fe), 45 (l, p). H. Aspöck \& U. Aspöck, 1971e: 108 (dis, rf), 110 (a, bio). Tjeder, 1972: 21 (lst). H. Aspöck \& U. Aspöck, 1976: 59 (tx). Monserrat \& Papenberg, 2010: 59 (rf). Monserrat \& Triviño, 2013: 15 (lst). Phaeostigma notata (Fabricius, 1781): H. Aspöck, 1986: 16, 17 (a). Hufnagel, 1987 (paleo). Pantaleoni, 1990a: 75 (dis). Pantaleoni, 1990b: 41 (p). Kielhorn, 1991 (dis). Poivre, 1991 (dis). Saure \& Gertsberger, 1991 (eco, dis). Plant, 1992: 119 (bio), 119, 127 (dis). Schmitz, 1993 (dis). Kleinsteuber, 1994 (dis, lapsus Huesca por Lérida). Plant, 1994: 17 (lst), 34, 35 (dis), 35 (bio). Pröse, 1995 (dis). Ševčik, 1995 (eco, dis, pa). Monserrat, 1996: 12 (1st). Röhricht, 1996: 138 (dis, 1st). Saure, 1996: 77-79 (bio, 1), 77, 80 (dis), 79 (fe), 80 (gen). Sziráki, 1996 (eco, dis). Sziráki \& Popov, 1996: 390 (dis). Achtelig, 1997: 49, 50 (dis). Gruppe, 1997: 27 (dis). Plant, 1997 (dis, tx). Tröger, 1997: 583 (1). Wachmann \& Saure, 1997 (eco, dis, tx). H. Aspöck, 1998a: 13 (1st), 14, 19, 24 (rf), 18 (tx), 26, 27 (mf). U. Aspöck \& H. Aspöck, 1999a: 18 (1). Sziráki, 1999: 144 (dis). Gruppe \& Schubert, 2001: 521, 522, 524 (bio), 520 
(dis). Plant, 2001: 183, 184 (dis), 184 (bio). Ábrahám, 2001: 257 (1st). Greve, 2002: 82 (c, 1), 84 (gen), 84, 86 (a), 85, 86 (dis), 86 (bio). Tröger, 2002: 38 (1st), 38, 39, 45 (dis), 41 (rf). Aistleitner \& Gruppe, 2004: 156 (dis). Gruppe et al., 2004: 246 (dis), 247 (bio). Dobosz et al., 2005: 73 (fe, 1, 1st). Pantaleoni, 2005: 76 (lst). Monserrat \& Triviño, 2013: 15 (lst).

Phaeostigma (Phaeostigma) notata (Fabricius, 1781): H. Aspöck et al., 1983a: 115 (dis), 117 (ㅇ). H. Aspöck et al., 1985a: 387 (pa). H. Aspöck et al., 1989: 74 (dis). H. Aspöck et al., 1991: vol. I: 28 (gen), 67-69, 191 (ð), 80, 192 (bio), 95, 97-101, 188, 192 (pa), 124, 125, 129, 131, 133, 134, 192, 193, 551, 556, 572, 573, 580, 581 (dis), 124, 127, 128, 131, 137, 138, 154, 157 (c), 150, 603 (rf), 185, 187, 191, 620 (a), 187, 190, 191 (tx), 191 (mf, + $), 192$ (fe, l), 612 (lst), vol. II: 50, 359 (mf), 55, 60 (a), 88, 92 (ð), 341, 442 (dis), 346 (1), 247 (ㅇ). Devetak, 1992b: 91 (lst), 103 (dis). Devetak, 1992c: 109 (dis). U. Aspöck et al., 1994a: 393, 394, 398, 399 (bio). Albertina et al., 1995: 5 (dis). H. Aspöck \& Hölzel, 1996: 40 (dis). Letardi \& Pantaleoni, 1996: 279 (dis). Pantaleoni \& Letardi, 1998: 17 (dis). Hölzel \& Wieser, 1999: 371 (dis), 425 (tx). H. Aspöck et al., 2001: 21 (tx), 21, 33, 327, (dis). H. Aspöck \& U. Aspöck, 2007: 97, 102, 104 (dis). Monserrat \& Papenberg, 2010: 48, 59 (tx), 51, 53, 58, 62 (ठ) $, 51,58,59$ (a), 53, 58, 63 (), 55 (c), 56 (1), 58 (bio mf), 58, 59 (pa), 59 (fe, 1st), 59-61, 63 (dis). Haring et al., 2011: 5 (dis, 1st), 10 (tx), fig.S2 (f). Monserrat \& Papenberg, 2012: 292 (rf). H. Aspöck \& U. Aspöck, 2013: 34 (1st), 66 (dis, o, , + ), 110 (eco), 136 (gen). Monserrat \& Triviño, 2013: 15, 43 (dis, 1st, tx).

= Raphidia megacephala Stephens, 1836: Curtis, 1824: 37 (rf). Stephens, 1829 (lst). Stephens, 1836: 130 (d). Curtis, 1837 (1st). Erichson, 1844 (rf). Hagen, 1850: 369 (rf). Hagen, 1858: 31 (a, dis, mf). McLachlan, 1868: 220 (sin). McLachlan, 1870: 18 (lst). Wallengren, 1871: 61 (sin). McLachlan, 1884 16 (rf). Selys-Longchamps, 1888: 160 (sin). Albarda, 1889 (sin). Albarda, 1891: 72 (sin). Kimmins, 1970: 356 (tx, mf). H. Aspöck \& U. Aspöck, 1976: 59 (tx), 62 (rf).

= Raphidia media Burmeister, 1839: Burmeister, 1839: 964 (d). Schneider, 1843: 76 (mf, mon). Hagen, 1844: 184 (dis), 184, 186 (a). Ratzeburg, 1844b (tx). Schneider, 1845: 252 (tx). Hagen, 1850: 370 (rf). Fischer, 1851 (1st). Walker, 1853: 209 (a, dis, mf). Brauer \& Löw, 1857: 53 (c, dis), 71 (lst). Hagen, 1860: 213 (dis, ie). Hagen, 1861 (ie). Wallengren, 1863: 16 (a). Hagen, 1867: 493 (rf), 495 (a, tx). McLachlan, 1868: 220 (sin). McLachlan, 1870: 18 (sin). Wallengren, 1871: 61 (sin). Meyer-Dür, 1875: 355 (c, dis, mf), 355, 356 (a), 356 (bio). Brauer, 1876: 266 (tx), 266, 277 (sin), 277 (dis). Brauer, 1878: 74 (lst). Girard, 1879: 491-521 (lst). Majewski, 1882: 15 (dis). Majewski, 1885: 12 (dis). Schoch, 1885: 93 (lst). SelysLongchamps, 1888: 160 (1st). Albarda, 1891: 77 (dis), 72-74, 76, 77, 80, 81 (rf). Banks, 1892: 357 (lst) - ie. Heyden, 1896: 111 (bio, p). Wanach, 1915: 324 (rf). Titschack, 1930: 114 (sin). Alfken, 1939: 517 (dis, sin). Bartoš, 1967: 341 (rf). H. Aspöck \& U. Aspöck, 1976: 59 ( $\sin )$.

= Raphidia angustata Ratzeburg, 1844: Ratzeburg, 1844a: 254 (d). Hagen, 1850: 370 (rf). Wallengren, 1863: 16 (sin). Hagen, 1867: 495 (a, tx). McLachlan, 1868: 220 (sin). SelysLongchamps, 1888: 160 (sin). Albarda, 1889 (sin). Albarda, 1891 (sin). H. Aspöck \& U. Aspöck, 1976: 59 (sin).

$=$ Raphidia laticeps Wallengren, 1871: Wallengren, 1871: $62(\mathrm{~d}$, mf): 63 (a, 1). Rostock, 1873 (rf). Brauer, 1876: 288 (dis). Brauer, 1878: 74 (1st). Rostock, 1881: 224 (dis). McLachlan, 1884: 16 (dis). Schneider, 1885: 24 (dis). Brauer, 1886: 25 (rf). Selys-Longchamps, 1888: 160 (1st). Rostock, 1888 (mf). Albarda, 1889 (sin). Albarda, 1891: 70 (mf), 81, 82 (rf) - (ie). Dziedzielewicz, 1891: 94 (lst). Reuter, 1894: 3 (rf). Wüstnei,
1902: 134 (dis), 143 (tx). Esben-Petersen, 1906: 45 (c), 46 (mf), 46, 47 (a), 47 (dis). Mjöberg, 1909: 156 (a, dis, mf). Schirmer, 1912: 140 (dis). Wanach, 1915: 324 (rf). Dziedzielewicz, 1920: 46 (dis). Lackschewitz, 1922: 19 (dis, ie). Tjeder, 1928: 162 (sin). Tjeder, 1937b: 120 (sin). Tjeder, 1940a: 98 (tx). Condé \& Pagès, 1954: 143 (rf). Condé \& Pagès, 1956a: 128 (rf). Metzger, 1960a: 199 (rf). Metzger, 1966: 38 (sin). H. Aspöck \& U. Aspöck, 1976: 59 (sin). Monserrat \& Triviño, 2013: 15 (lst). Raphidia notata var. laticeps Wallengren, 1871: Esben-Petersen, 1929 (mf). Weiss, 1947 (mf). Ohm, 1961b: 2 (a). Monserrat \& Triviño, 2013: 15 (lst).

=Erma abdita Navás, 1918: Navás, 1918a: 20 (d), 20-22 (mf), 21, 22 (a). Navás, 1918b: 43 (dis, rf). Navás, 1919: 82, 83 (a, mf), 83 (dis). Navás, 1923: 73 (dis). Lestage, 1928: 30 (rf). Navás, 1928a: 41, 42 (mf), 42 (a), 43 (dis). Navás, 1933a: 121 (1st). Navás, 1934a: 6 (dis). Navás, 1934b: 48 (mf, ô), 48, 49 (a), 49 (dis). Carpenter, 1936: 93 (rf). H. Aspöck \& U. Aspöck, 1965: 363 (rf). H. Aspöck \& U. Aspöck, 1971a: 7 (rf). H. Aspöck \& U. Aspöck, 1971e: 107, 108, 111 (tx). H. Aspöck \& U. Aspöck, 1976: 59 (sin), 61 (a). Monserrat \& Papenberg, 2010: 48 (tx), 49, 59 (ref). Monserrat \& Triviño, 2013: 15 (lst).

= Lesna navasi Steinmann, 1963: Steinmann, 1963: 189 (d), 189, 191, 194 (a), 195 (c), 189-191 (mf), 190, 191 (ㅇ). H. Aspöck \& U. Aspöck, 1966a: 47 (sin). Steinmann, 1967 (rf). H. Aspöck \& U. Aspöck, 1969a: 35 (rf). H. Aspöck \& U. Aspöck, 1976: $59,62(\sin )$.

= Lesna lestica Steinmann, 1963: Steinmann, 1963: 190 (d), 190-192 (mf, †), 196 (a), 195 (c). H. Aspöck \& U. Aspöck, 1966a: 47 (sin). Steinmann, 1967 (mf). H. Aspöck \& U. Aspöck, 1976: 59, 62 (sin).

= Lesna stigmata Steinmann, 1963: Steinmann, 1963: 192 (d), 192, 193, 195 (P), 193, 194, 196 (a), 197 (c), 193-195 (mf). H. Aspöck \& U. Aspöck, 1966a: 47 (sin). Steinmann, 1967 (rf). H. Aspöck \& U. Aspöck 1969a: 36 (rf). H. Aspöck \& U. Aspöck, 1976: 59 (tx), 62 (sin).

= Raphidia stigmata (Steinmann, 1963): Bartoš, 1964: 320 (dis).

= Lesna laticaput Steinmann, 1963: Steinmann, 1963: 192 (d), 193 (mf), 196 (a). H. Aspöck \& U. Aspöck, 1966a: 44 (dis). Bartoš, 1967: 341 (rf). Steinmann, 1967 (rf). H. Aspöck \& U. Aspöck, 1969a: 35 (rf). H. Aspöck \& U. Aspöck, 1976: 59, 62 (sin).

= Raphidia laticaput (Steinmann, 1963): Bartoš, 1964: 320 (dis).

= Navasana perumbrata Steinmann, 1963: Steinmann, 1963: 186 (d), 187 (a, mf). H. Aspöck \& U. Aspöck, 1966a: 44 (dis). Steinmann, 1967 (rf). H. Aspöck \& U. Aspöck, 1969a: 35 (rf), 36 ( $\sin )$. H. Aspöck \& U. Aspöck, 1976: 59 (sin).

Raphidia ophiopsis Linnaeus, 1758: Curtis, 1824: 37 (mf) - ie. Stephens, 1836: 130 (mf) - ie. Zetterstedt, 1840: 1054 (mf) - ie. Navás, 1911b: 36 (c, mf) - ie. Navás, $1913 \mathrm{c}: 107$ (lst) - ie.

TIPO: neotipo $\hat{\sigma}$ depositado en el Natural History Museum (Londres, Reino Unido).

LOCALIDAD TÍPICA: Surrey, Croydon, Coombe Hurst (Inglaterra).

MATERIAL ESTUDIADO: ESPAÑA: Huesca: Benasque, Llanos del Hospital, 31TCH2846, 1.757 m, 17.VII.09, 1 q sobre hierba, V. J. Monserrat (VM).

MORFOLOGÍA EXTERNA: Es una especie grande y robusta. La cabeza es grande, ensanchada en su base, de coloración negra con una banda media parda. El clípeo es negro y el labro pardo oscuro. El escapo es pardo y el flagelo pardo oscuro y en su base pardo ocre. El pronoto es corto, robusto, de coloración parda 
y en el margen latero-posterior está bordeado por una banda estrecha amarilla, su borde porta setas blancoamarillentas. Las coxas, los trocánteres y el fémur III son negros, pudiendo presentar un tono pardo. El fémur I y II en la cara interna de la región basal y en la externa son pardo oscuros, el resto es pardo ocre. Los demás artejos adquieren diferentes tonalidades, de amarillo-ocre hasta pardo-ocre. Las coxas, trocánteres y fémures portan penachos de setas blanco amarillentas.

La longitud del ala anterior es de entre $9-13 \mathrm{~mm}$

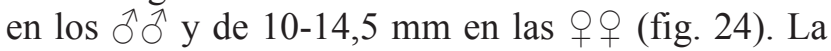
venación alar es parda oscura. Solo en la base del ala aparecen venas amarillas, siendo en el ala anterior aisladas y en la posterior de forma mayoritaria. El pterostigma es pardo, de longitud desde corta a media, atravesado por dos venas, y de forma minoritaria sólo por una y que suele estar bifurcada. El ala anterior suele tener cuatro celdas postigmales. La parte basal de la $\mathrm{Ma}$ en el ala posterior transcurre como vena longitudinal (fig. 24).

SEGMENTOS GENITALES: En los $\widehat{\partial} \hat{\jmath}$ el proceso del gonocoxito se encuentra orientado en dirección ventral (fig. 43). Aparece un gonarco (fig. 44). Los parámeros se encuentran unidos mediante un puente y sus extremos caudales mantienen una posición paralela (fig. 93).

En la $\varnothing$ la subgenitalia está desarrollada como una estructura compacta (figs. 100, 101). El ductus saculi es corto (figs. 124, 125). El saco de la bolsa copuladora es de tamaño medio. El ductus receptaculi parte del extremo anterior de la bolsa (fig. 124). El receptáculo seminal es piriforme con una fina estriación. Las glándulas son cortas y filiformes (fig. 124).

BIOLOGÍA: Existen numerosos registros sobre Pinus, Picea, Larix, Abies, Pyrus, Malus, Quercus, Aesculus, Alnus, Acer, Prunus, Betula y Sambucus. Es una especie eurioica, y en Europa Central se registran en todo tipo de bosques, sean de coníferas, de ribera, robledales o frutales. Esta especie suele evitar áreas secas y cálidas, siendo característica de los bosques de coníferas subalpinos, pudiendo aparecer altas densidades de individuos en sus poblaciones. En la península solo hemos podido estudiar un ejemplar recolectado sobre hierba, pero anotamos la información fiable existente en la bibliografía. Se ha citado desde el nivel del mar hasta el piso alpino, en algunas zonas de los Alpes meridionales hasta los $2.000 \mathrm{~m}$ de altitud, en España a $1.757 \mathrm{~m}$. En varias especies del género se han aislado parasitoides del género Nemeritis y Tropistes (Hymenoptera: Ichneumonidae), y en Ph. notatum se han localizado parásitos y parasitoides: Gregarinidae: Gregarina raphidiae Acht. (Alemania meridional). Hymenoptera: Nemeritis caudatula Thoms. (Austria, Suiza, Montenegro), Nemeritis specularis specularis Horstm. (Austria), Nemeritis silvicola Horstm. (Austria), Tropistes falcatus Thoms (Austria), e hiperparásitos:
Perilampus polypori Bouček (Austria) (H. Aspöck et al., 1991; H. Aspöck 2002).

FENOLOGÍA: La actividad de los imagos se desarrolla durante los meses de IV a VIII, en España en VII.

VARIABILIDAD: En general la variabilidad es escasa. Las cuatro celdillas postigmales se encuentran frecuentemente incompletas, pudiendo faltar en raras ocasiones. Existe una gran uniformidad en la genitalia

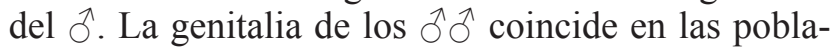
ciones de los Pirineos, de Montenegro, así como del oeste de Rusia o de Europa Central.

ESTADIOS PREIMAGINALES: Las larvas son probablemente corticícolas y subcorticícolas, existen numerosos registros de larvas en cortezas de angiospermas y gimnospermas, mostrando una clara preferencia por las coníferas (Pinus, Picea, Larix, Abies), aunque también se han citado sobre encinas, robles y frutales como perales o manzanos. El periodo de desarrollo de larvas criadas en cautividad es, como mínimo, de dos años, y en parte de ellas al menos tres. La última fase de hibernación transcurre en fase de larva (Tipo I). La fase de pupa (en cría) se mantiene de 13-18 días. Morfología y aspecto de la larva en figura 144 y en H. Aspöck et al. (1991).

DISTRIBUCIÓN: Europa: Austria, Bélgica, Bielorrusia, Suiza, República Checa, Alemania, Dinamarca, España, Estonia, Francia, Liechtenstein, Reino Unido, Hungría, Croacia, Italia, Lituania, Letonia, Noruega, Holanda, Polonia, Rumania, Rusia, Suecia, Eslovaquia, Eslovenia, Ucrania, Yugoslavia. Las únicas citas que se conocen de España están situadas en el noreste de la Península Ibérica (fig. 156).

Phaeostigma (Phaeostigma) italogallicum (H. Aspöck \& U. Aspöck, 1976)

Raphidia (Phaeostigma) italogallica H. Aspöck \& U. Aspöck, 1976: H. Aspöck \& U. Aspöck, 1976: 65-69 (d). H. Aspöck et al., 1980a: vol. I: 41, 43 (c, tx), 98 (a, bio, 1), 98, 349 (dis), vol. II: 160 (a), 25 (ð̋), 42 (ㅇ), 237 (dis). Pantaleoni, 1986: 168 (dis). Pantaleoni, 1988: 634 (lst). Cocquemont \& Chambon, 1990: 135 (lst). Stelzl, 1991: 471, 472 (bio). Monserrat \& Triviño, 2013: 15 (1st).

Phaeostigma (Phaeostigma) italogallica (H. Aspöck \& U. Aspöck, 1976): H. Aspöck et al., 1983a: 114, 115 (dis), 117 (ㅇ). H. Aspöck et al., 1985: 387 (pa). H. Aspöck et al., 1991: vol. I: 86, 194, 195 (bio), 97, 98, 195 (pa), 125, 127, 129, 131, $133-135,137$ (c) $, 129,131,133,134,192,195,550,572,573$ (dis), 135 (rf), 187, 190, 191 (tx), 188 (ð), 194 (a, mf, 1), 195 (fe), 612 (lst), vol. II: 48 (h), 60 (a), 89, 92 (ठ), 248 (q), 443 (dis). Pantaleoni, 1993: 288 (dis). Pantaleoni et al., 1994: 127, 174 (dis). Albertina et al., 1995: 5 (dis). H. Aspöck H \& Hölzel, 1996: 40 (dis). Letardi \& Pantaleoni, 1996: 279 (dis). Pantaleoni \& Letardi, 1998: 17 (dis). H. Aspöck et al., 2001: 21 (tx), 22, 327, 333 (dis). Rausch et al., 2004: 164 (gen). H. Aspöck \& U. Aspöck, 2007: 97, 104 (dis), 102 (rf). Monserrat \& Papenberg, 2010: 48 (rf), 48, 60, 61, 63, 64 (dis), 51, 53, 62, 63 (ð) $, 51,62$ (a). 53, 62, 63 (), 55 (c), 56, 62, 63 (1), 59 
(tx), 62 (bio, fe, mf). Haring et al., 2011: 5 (dis, lst) 10 (tx), fig.S2 (f). Ocharan et al., 2012: 423 (bio, dis). Monserrat \& Papenberg, 2012: 292 (rf). H. Aspöck \& U. Aspöck, 2013: 34 (lst), 131 (gen). Monserrat \& Triviño, 2013: 15, 43 (dis, 1st, tx). Raphidia (Navasana) sp.: H. Aspöck \& U. Aspöck, 1971: H. Aspöck \& U. Aspöck, 1971e: 109, 110 (ð), 110 (dis), 111 (†), 108 (rf).

TIPO: holotipo $\widehat{\partial}$ depositado en la Colección Aspöck.

LOCALIDAD TÍPICA: Norte de Sisteron, Provenza (Francia).

MATERIAL ESTUDIADO: ESPAÑA: Asturias: Muniellos, Laguna de la Isla, 29TPH8464, 1.340 m, 20.VI.2001, 1 o, R. Ocharan (VM). Guipúzcoa: Lareo, Enirio, Aralar, Parque Natural, 30TWN7259, 750 m, 16.VI.2005, 1 o sobre Salix atrocinerea, S. Pagola Carte (VM). Navarra: Arangoiti, Tontorra, Irunberri, 30TXN42, 1.353 m, 23.V.2003, 1 ô, S. Pagola Carte (VM). Lérida: Barranco de Ortedó, 31TCG7788, $1.100 \mathrm{~m}$ 19.VI.1991, 2 우 sobre Pinus radiata, (a*, $\left.{ }^{*}\right), 1$ larva ex ovo, V. J. Monserrat (VM).

MORFOLOGÍA EXTERNA: Es una especie grande y robusta. La cabeza es grande y larga, de coloración negra con una banda media central rojo oscura-parda. El clípeo y el labro son negros. El escapo y el pedicelo pardo ocre. El flagelo es pardo, siendo su base parda ocre. El pronoto es corto y robusto, pardo oscuro bordeado en su parte posterior con una banda ancha y clara que presenta penachos de setas blancas. Las coxas, los trocánteres y los fémures del III par de patas son pardo oscuros. El fémur I y II en su parte basal interna y en su cara externa son pardo oscuros, las otras zonas del fémur, al igual que los restantes artejos, son pardo ocre. En las coxas, trocánteres y fémures aparecen penachos de setas blancas.

La longitud del ala anterior es de entre 9-11 mm en los $\widehat{\partial} \hat{\partial}$ y de 10-13 mm en las $9+$ (fig. 25). La venación alar es parda, apareciendo regiones ocres en la base del ala (ala anterior) o venas ocres (ala posterior). El pterostigma es pardo, de longitud media y está atravesado por una vena que se bifurca en su base (fig. 25). El ala anterior presenta tres celdillas postigmales, pero se ha observado en un ejemplar del País Vasco cuatro celdillas. La parte basal de la Ma del ala anterior transcurre como vena longitudinal (fig. 25). En el abdomen los terguitos y esternitos son negros, teniendo estos últimos un borde caudal pardo ocre.

SEGMENTOS GENITALES: En los $\widehat{\jmath}$ el proceso del gonocoxito se encuentra orientado en dirección dorsal y presenta una espina suplementaria con la misma orientación (figs. 45, 46). Los parámeros se encuentran unidos por un puente y sus extremos caudales se entrecruzan (fig. 94).

En las $ㅇ$ 오 la subgenitalia está desarrollada como estructura compacta de coloración clara (fig. 118). La bolsa copuladora sigue el esquema general de Phaeostigma s.str., siendo su morfología muy parecida a la de la especie Phaeostigma notatum. Las glándulas del receptáculo seminal son cortas.
BIOLOGÍA: Las citas se sitúan alrededor de los $500 \mathrm{~m}$ (sur de Francia), 900-1.550 m (Italia) o entre los 750$1.353 \mathrm{~m}$ (España). Citada sobre Abies, Pinus (los ejemplares ibéricos sobre Pinus radiata y sobre Salix en biotopos relativamente templados). A veces sus densidades de población pueden ser localmente altas.

FENOLOGÍA: La actividad de los imagos se desarrolla durante los meses de V a VII. En España ha sido hallada en V y VI.

VARIABILIDAD: En general la variabilidad es escasa.

ESTADIOS PREIMAGINALES: Es de suponer que las larvas son probablemente corticícolas o subcorticícolas. Hay registros de larvas de esta especie sobre Abies, Quercus y Pinus. El periodo de desarrollo de larvas criadas comprende entre dos y tres años. La última fase de hibernación transcurre en fase de larva (Tipo I). La fase de pupa (en cría) se mantiene de 9-17 días.

DISTRIBUCIÓN: Sur de Francia, sur de Italia y noreste de España (fig. 157).

DISCUSIÓN: Una clara diferenciación entre las especies Phaeostigma notatum y Phaeostigma italogallicum realmente sólo es posible en los $\widehat{\partial} \widehat{\partial}$ y mediante el estudio de su genitalia. Las 90 aisladas se diferencian con dificultad (figs. 124, 125). En la ${ }^{\circ}$ existen diferencias en la morfología del $8^{\circ}$ terguito (figs. 100-102) o más claras en la forma de la subgenitalia (figs. 100, 118). En la venación alar existe una diferenciación en el número de celdas postigmales, que en el caso de Phaeostigma notatum es de 4 (fig. 24), mientras que en Phaeostigma italogallicum suele ser de 3 (fig. 25), apareciendo excepciones como en el ejemplar de Navarra, $\hat{\jmath}$ (Arangoiti, Tontorra, Irunberri) que posee 4 celdillas postigmales o el ejemplar de Asturias, ô (Muniellos, Laguna de la Isla) que presenta el pterostigma atravesado por dos venas paralelas y en una de las alas aparecen cuatro celdillas postigmales, al igual que en la especie $P h$. $(P h$.) notatum, lo que hace que este carácter tenga un valor taxonómico relativo. Comparando la morfología de los individuos de la Península Ibérica con los descritos de Francia e Italia coinciden prácticamente en su totalidad.

\section{Género Subilla Navás, 1916}

Subilla Navás, 1916: 509 (d, a), [especie tipo por designación original: Raphidia sericea Albarda, 1891 y por sinonimias posteriores corresponde a: Raphidia confinis Stephens, 1836=(Raphidia cognata Rambur, 1842=Raphidia sericea Albarda, 1891)].

Considerado como subgénero de Raphidia Linnaeus, 1758: H. Aspöck \& U. Aspöck, 1968b: 62 (tx). H. Aspöck \& U. Aspöck, 1972a: 33, 34 (tx), 40, 41 (c), 41, 42 (bio). H. Aspöck \& U. Aspöck, 1974c: 178, 180 (rf). H. Aspöck et al., 1975: 368 (bio), 372 (1). Popov, 1977: 272 (a, ㅇ, tx). H. Aspöck, 1979: 249, 255 
(dis). H. Aspöck et al., 1980a: vol. I: 86, 87 (dis), 94, 123 (tx), 114 (rf), vol. II: 250 (dis).

Considerado como género: Navás, 1916: 509 (a, d). Navás, 1919: 6 (dis), 12 (c). Banks, 1923: 115 (rf). Navás, 1925: 43 (c). Lestage, 1928: 30, 31 (rf). Navás, 1934b: 13, 20 (c), 20 (a). Carpenter, 1936: 93 (tx). Steinmann, 1963: 183 (1st), 185 (c). Bartoš, 1965a: 93 (rf). Bartoš, 1967: 334 (a), 335 (rf). Buresch \& Popov, 1973: 155 (rf). H. Aspöck et al., 1982: 85 (1, tx). H. Aspöck et al., 1984: 101 (tx). H. Aspöck, 1986: 23 (tx), 25 (dis). H. Aspöck, 1987: 152, 156 (dis). H. Aspöck et al., 1989: 88, 101 (dis). H. Aspöck et al., 1991: vol. I: 54, 76, 83, 276, 277 (bio), 114, 276, 558, 611, 618 (dis), 275 (a, mf, ふૈ), 276 (p, pa, + ), 105, 111, 113, 275 (tx), vol. II: 418 (dis). Oswald \& Penny, 1991: 66 (lst). H. Aspöck \& Hölzel, 1996: 38 (dis). H. Aspöck et al., 2001: 20, 38 (tx), 38 (dis). H. Aspöck, 2002: 38, 44-46 (1, pa). Pantaleoni et al., 2004: 3, 4 (rf). H. Aspöck \& U. Aspöck, 2007: 98, 103 (dis). 100 (rf), 103 (gen). Letardi \& Pantaleoni, 2007: 89 (1st). Monserrat \& Papenberg, 2010: 63 (gen). U. Aspöck et al., 2012: 577 (1st), 579 (f), 580 (rf). Liu et al., 2012: 239 (ठ). H. Aspöck \& U. Aspöck, 2013: 12 (tx), 42 (gen).

Actualmente el género Subilla engloba en total diez especies de distribución mayoritariamente europea. En la península está representado por Subilla aliena.

\section{Subilla aliena (Navás, 1915)}

Raphidilla aliena Navás, 1915: Navás, 1915c: 200, 201 (d). Navás, 1915a: 792 (dis). Navás, 1915b: 873 (dis). Fuente y Morales, 1929: 167 (dis, ô, \&), 167, 168 (a, mf). Bartoš, 1965a: 96 (a, §), 98 (tx). Martín Albaladejo \& Izquierdo Moya, 2006: 134 (1st). Monserrat \& Triviño, 2013: 15 (lst).

Raphidia aliena (Navás, 1915): Navás, 1919: 37 (c), 48 (a, mf, đ̂̃, O). Navás, 1924: 130 (dis). Navás, 1928a: 18, 19 (mf), 19 (a, dis, đ). Navás, 1933a: 121 (1st). Navás, 1934a: 5 (dis). Navás, 1934b: 23 (c), 27 (mf), 27, 28 (a), 28 (dis, đ̋). Navás, 1935: 73 (rf), 74 (c). Steinmann, 1964: 201 (a), 202 (c). H. Aspöck \& U. Aspöck, 1966a: 46 (rf). H. Aspöck \& U. Aspöck, 1968b: 62 (tx). H. Aspöck \& U. Aspöck, 1968d: 22 (†), 22, 24 (ふ), 23 (rf), 24, 25 (dis), 24, 26 (tx). H. Aspöck \& U. Aspöck, 1971e: 109 (rf). Monserrat \& Triviño, 2013: 15 (lst).

Raphidia (Subilla) aliena (Navás, 1915): H. Aspöck \& U. Aspöck, 1968bc: 62 (tx). H. Aspöck \& U. Aspöck, 1971a: 7 (rf). H. Aspöck \& U. Aspöck, 1972a: 34 (tx), 35-37, 41 (dis), 36, 42 (bio), 40 (c). Popov, 1973: 121 (a, rf). H. Aspöck \& U. Aspöck, 1974b: 111 (dis). H. Aspöck et al., 1980a: vol. I: 38 (c), 115 (bio, 1, tx), 115, 351 (dis), vol. II: 35 (ふ) $), 165$ (a), 250 (dis). Díaz-Aranda et al., 1986: 1134 (bio, dis). Monserrat \& DíazAranda, 1987: 175 (rf). Díaz-Aranda \& Monserrat, 1988a: 218 (dis). Díaz-Aranda \& Monserrat, 1988b: 214-215 (dis). Monserrat \& Triviño, 2013: 15 (lst).

Subilla aliena (Navás, 1915): H. Aspöck et al., 1985a: 387 (pa). H. Aspöck et al., 1991: vol. I: 88 (bio), 97, 98, 100 (pa), 129, 551, 574, 575 (dis), 280 (a, mf, ô, o + ), 275, 279, 280 (tx), vol. II: 57, 69 (a), 135, 144 (§), 350 (1), 464 (dis). Poivre, 1991 (dis). Marín \& Monserrat, 1995: 111 (lst). H. Aspöck \& Hölzel, 1996: 42 (dis). Monserrat, 1996: 12 (lst). Pantaleoni \& Letardi, 1998: 17, 18 (dis). H. Aspöck et al., 2001: 38 (tx), 38, 330, 336 (dis). Martín Albaladejo \& Izquierdo Moya, 2006: 134 (dis, 1st). H. Aspöck \& U. Aspöck, 2013: 35 (lst), 44 (gen). Monserrat \& Triviño, 2013: 15, 44 (dis, 1st, tx).

= Raphidia oteroi Navás, 1935: Navás, 1935: 71 (d), 71-73 (mf), 72, 73 (a), 74 (c). H. Aspöck \& U. Aspöck, 1968d: 23 (rf). H. Aspöck \& U. Aspöck, 1972a: 35 (sin). Monserrat, 2013: 288 (bio, dis, 1st). Monserrat \& Triviño, 2013: 15 (lst).
Raphidia cognata Rambur, 1842: Pictet, 1865: 53, 54 (mf), 54 (a, dis) - ie. Brauer, 1876: 288 (rf). Navás, 1905a: 38 (dis, 1st, mf) - ie. Navás, 1908: 131 (c, dis) - ie. Navás, 1911b: 36 (c, mf) - ie. Navás, 1913c: 107 (lst) - ie. Navás, 1915b: 874 (dis) - ie. Navás, 1919: 46 (rf) - ie. Navás, 1928a: 16 (a, mf, rf) - ie. Navás, 1934b: 29 (rf) - ie. Navás, 1935: 74 (c, rf) - ie. Steinmann, 1964: 201 (rf). H. Aspöck \& U. Aspöck, 1966a: 46 (rf). Bartoš, 1967: 333 (rf). Monserrat \& Triviño, 2013: 15 (lst). Raphidia (Raphidia) cognata Rambur, 1842: Berland, 1962: 18 (mf, rf).

Raphidia (Subilla) cognata Rambur, 1842: Séméria \& Berland, 1988: 32 (rf).

TIPO: lectotipo $q$ depositado en el Museo de Barcelona. Lectotipo ô Raphidilla aliena Navás 1915 H. Aspöck \& U. Aspöck vid. 74.

LOCALIDAD TÍPICA: Provincia de Madrid (España).

MATERIAL ESTUDIADO: ESPAÑA: Alicante: Sax, 30SXH9067, 470 m, 28.VI.1982, 1 q sobre Pinus halepensis, V. J. Monserrat (VM). Ávila: Ojos Albos, 30TUL7007, 1.226 m, 14.VI.1977, $1 \hat{\sigma}$ sobre P. sylvestris, V. J. Monserrat (VM). Cáceres: La Granja, 30TTK5658, 430 m, 18.V.1980, 1 o sobre Quercus ilex, V. J. Monserrat (VM), Oliva de Plasencia, 29TQE4844, $400 \mathrm{~m}$, 18.V.1980, 2 ऊิ के sobre Q. ilex, V. J. Monserrat (VM), Puerto de Covacha, 29SQD0741, 380 m, 19.V.1977, 1 sobre Q. suber, V. J. Monserrat (VM), Rio Salor, 29SQD1663, 330 m, 17.V.1980, 1 q sobre Q. ilex, V. J. Monserrat (VM), Venta de la Calleja, 29SQD1147, 350 m, 17.V.1980, 1 đ sobre Q. suber, V. J. Monserrat (VM). Cádiz: Estación de la Almoraima, 30STF8117, 25.V.2012, 1 q sobre Q. canariensis, V. J. Monserrat (VM). Coruña: Santiago de Compostela, 29TNH3747, 260 m, 22.V.1981, 1 , F. Novoa (VM). Cuenca: Uña, 30TWK8753, 1.146 m, 28.V.1986, 1 ڤิ sobre Q. faginea, A. Baz (VM). Granada: Arenales, 30SVG43, 783 m, 1.V.1999, 1 ते sobre Olea europea, EEZ, leg.? (EEZ), Collado de la Sabina, Sierra Nevada, 30SVG60, 2.200 m, 30.VI.2011, 1 đิ sobre P. sylvestris, V. J. Monserrat (VM), 10.VII.2012, 1 ๙ sobre P. sylvestris, V. J. Monserrat (VM), Puerto de los Alazores, 30SVF8899, $1.100 \mathrm{~m}, 1 . \mathrm{VI} .1986,1$ \%, V. J. Monserrat (VM), Puerto de Onitar, 30SVG4544, $900 \mathrm{~m}, 1$.VI.1986, 1 đ sobre Q. ilex, leg.? (VM), Trévelez, 30SSVF7695, 1.476 m, VII.1974, 1 गे, A. Compte (MNCN), Zaidín, Parque de Invierno, 30SVG4612, 1.000 m, 22.VI.1986, 1 \%, M. Campos (VM). Guadalajara: Aldeanueva de Atienza, 30TVL9257, $1.311 \mathrm{~m}, 28$. VI.1989, 1 ते sobre P.sylvestris, V. J. Monserrat (VM), Arroyo Fraguas, 30TVL8555, 1.217 m, 29.VI.1984, 1 đ̊ sobre Q. ilex, L. M. Díaz-Aranda (VM), Valdenuño, 30TVL6812, 800 m, 29.VI.1984, 1 q sobre Q. ilex, V. J. Monserrat (VM). Guipúzcoa: Ataun, Aia, Aralar, Parque Natural, 30TWN6858, 614 m, 28.VI.-8.VII.2011, 1 q en trampa de atracción sobre Q. ilex ilex, S. Pagola Carte (VM). Huesca: Valle de Ordesa, 8.V.-3.VII.2000, 1 o / 2 우 en trampa de Malaise, 30TYN3826, 26.VI.1966, 1 †, M. Ortego (VM). Jaén: Cañada Morales, 30SWH2129, $1.200 \mathrm{~m}, 4 . \mathrm{VI} .1986,1$ q sobre Q. ilex, V. J. Monserrat (VM), Isabella, 30SVH4933, 600 m, 4.VI.1986, 1 우 sobre $Q$. ilex, V. J. Monserrat (VM), Sierra de Cazorla, Arroyo de Linarejos, 30SWG0797, 22.VI.1984, 1 으, J. Alba (VM), Sierra de Cazorla, Camino de Agracea, 30SWH1849, 29.V.1991, 1 đै sobre Phillirea latifolia, 1 q sobre Crataegus monogyna, J. L Yela (VM), Hoyo de Muñoz, 30SWH3351, 830 m, 13.V.1991, 1 ô sobre Rosa sp., J.L. Yela (VM), Sierra Morena, Monte Selladores, 30SVH2747, 700 m, 31.V.1986, 1 + sobre Q. ilex, V. J. Monserrat (VM). León: León, 30TN81, 838 m, 18.VII.1982, 1 , A. M. Vega (UNIL). Madrid: Aluche, 30TVK3470, 680 m, 2.VI.2004, 1 ô, M. Costas (VM), Batres, 30TVK2151, 600 m, 15.V.1978, 1 ô sobre O. europea, V. J. Monserrat (VM), Boadilla del Monte, 30TVK2573, 689 m, 25.VI.2005, 1 , D. Papenberg (VM), 5.VII.2005, 1 ㅇ, D. Papenberg (VM), Ciudad Universitaria, 30TVK3877, 700 m, 
4.VI.1975, 1 ๙, 12.V.2008, 1 ๙ , V. J. Monserrat (VM), 17.VI.1975, 1 +, V. J. Monserrat(VM), 27.VI.1979, 1 + , F. Martín(VM), 6.VI.2010, 1 ․ V. J. Monserrat (VM), Chamartín, 30TVK48, 4.VII.1978, 1 q, Munguira (UAM), El Escorial, 30TVK0293, 909 m, 4.IV.1977, 1 , P. Villanueva (VM), Fuente del Cervunal, Abantos, 30TVK0296, 1.700 m, 21.VI.2008, 1 o sobre P. sylvestris, V. J. Monserrat (VM), El Pardo, 30TVK3481, 620 m, VI.1908, 1 ㅇ, Arias (MNCN), El Plantío, 30TVK28, 667 m, 19.V.1991, 1 đ̃, G. Díez (UAM), Hoyo de Manzanares, 30TVK2397, 950 m, 18.VI.1975, 1 q sobre hierba, (a*, (*), V. J. Monserrat (VM), Las Matas, 30TVK2489, 750 m, 8.V.1992, 1 q, I. Marcos (MNCN), Las Rozas, 30TVK2683, 718 m, 10.VI.1978, 1 ․, M. Gallardo (VM), 26.III.2013, 1 9 posada en el interior de una casa, P. Cebrián (VM), Madrid, 30TVK37, 655 m, 19.V.1978, 1 đ̃, J. Templado (VM), Madrid, Chamartín, 30TVK48, 4.VII.1978, 1 क , Munguira (UAM), Madrid, Parque del Oeste, 30TVK7639, V, 1 ㅇ, leg.? (VM), Majadahonda, 30TVK28, 743 m, 2.V.1982, 1 9, E. Ochoa (MNCN), Soto del Real, 30TVL3312, 921 $\mathrm{m}, 14$. VI.1993, 1 ${ }^{\lambda}$, Munguira (UAM), Torrelaguna, Valgallegos, 30TVL5419, 744 m, 13.III.1998, 1 đ en agalla de $Q$. faginea, C. Ornosa (VM). Málaga: Alfarnate, 30SUF8795, 886 m, 1.VI.1986, 1 ô sobre Q. ilex, leg.? (VM), Ronda, Sierra de las Nieves, 30SUF196, 21.VI.1997, 1 đै, J. de Ferrer (MNCN). Navarra: Isu-Biotzari, Erromantzatua, 30TXN52, 720 m, 23.V.2003, 1 ㅇ, S. Pagola Carte (VM). Orense: Herosa, 29TPG5052, 880 m, 6.VI.1977, 1 ․ , V. J. Monserrat (VM). Salamanca: Casillas de Flores, 29TPE9072, 880 m, 24.IV.1977, 1 + sobre Q. pyrenaica, V. J. Monserrat (VM), El Palacio, 20.V.1977, 1 \& sobre Q. ilex, V. J. Monserrat (VM), Milano, 29TQF0151, 750 m, 7.V.1977, 1 § sobre Cytisus multiflorus, V. J. Monserrat(VM). Segovia: La Granja de San Ildefonso, 30TVL1528, 1.193 m, VII.1944, 1 क , S.V. Peris (VM), Ortigosa, 30TVL0022, 1.166 m, 29.V.1955, 1 ㅇ, S.V. Peris (VM), Valsaín, 30TVL1326, 1.180 m, 15.VI.1993, 1 ふै, leg.? (VM). Teruel: Monterde de Albarracín, 30TXK2884, 1.300 m, 24.VI.1986, 1 q sobre Juniperus thurifera, V. J. Monserrat (VM), Puerto de Cuarto Pelado,

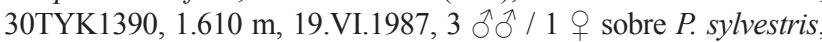
V. J. Monserrat (VM). Toledo: Sierra de Pelahustán, 30TUK6348, 700 m, 4.VI.1978, 1 o sobre Q. ilex, V. J. Monserrat (VM), Urda, Base Infoc., 30SVJ3863, 763 m, 2.VI.201, 1 o sobre Tamarix gallica, V. Triviño (VM). Zamora: Valparaíso, 30TTL7180, $800 \mathrm{~m}$, 15.V.1977, 1 q sobre Q. faginea, V. J. Monserrat (VM). Zaragoza: Fuendetodos, 30TXL7070, 15.V.2006, 1 त sobre Q. ilex, V. J. Monserrat (VM), Tabuenca, 30TXM2220, 3.VI.1994, 1 ㅇ sobre $Q$. ilex, F. Marín (VM). PORTUGAL: Beira Alta: Figueira de Castelo, Rodrigo, 29TPF7127, 786 m, 24.VI.2008, 1 ㅇ, V. J. Monserrat (VM). Braga: Bom Jesus, 29SMD84, VI.1899, 1 +, leg.? (FCTUC).

MORFOLOGÍA EXTERNA: Es una especie de tamaño pequeño o mediano. La cabeza tiene una sutura central de color pardo. El clípeo y el labro son pardos. Las antenas en su base son amarillentas y en su región apical son pardo oscuras. El color del pronoto va desde negro hasta pardo oscuro, siendo algo más claro en la región anterior, y su borde presenta una banda ancha amarilla en su parte posterior y ocre en la anterior. En los apéndices locomotores, las coxas II y III son pardo oscuras. La coxa I, los trocánteres y el fémur III son pardos. La coxa I de forma individual, en algunos casos, puede ser amarilla en su zona anterior. El fémur II y frecuentemente el fémur I, presenta en su cara exterior una banda longitudinal parda. El resto de los apéndices son amarillentos.

La longitud del ala anterior es de $6.8-8.5 \mathrm{~mm}$ en los

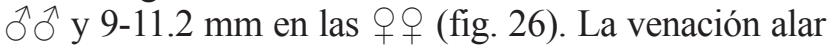

presenta una tonalidad parda. La costa, la región proximal del radio, así como venas aisladas situadas en la base del ala son amarillentas. El pterostigma es amari1lo, frecuentemente ocre-amarillento. La parte basal de la Ma en el ala posterior se encuentra frecuentemente acortada y tiene un recorrido longitudinal (fig. 26). En el abdomen los terguitos y esternitos son pardo oscuros.

SEGMENTOS GENITALES: En los $\hat{\partial} \widehat{\partial}$ los segmentos genitales son llamativamente amarillos. El estilo es grande, con un lóbulo en posición dorsal basal plano formando con el estilo un ángulo de $90^{\circ}$ (figs. 47, 48). Los parámeros son externamente dentados y se encuentran unidos en posición anterior mediante un puente (fig. 95). El hipandrio interno es mediano (fig. 81). El ectoprocto es grande (fig. 47).

En la $q$ el $7^{\circ}$ esternito es amarillo parduzco. El intersegmento $S \mathrm{~T} / 8$ es largo y profundo con unos salientes esclerotizados característicos (figs. 103, 104). Genitalia interna según fig. 126.

BIOLOGÍA: Las especies del género Subilla muestran una preferencia por biotopos colonizados por Quercus spp., y también en la península los imagos parecen tener preferencia a colonizar biotopos xerotermos en bosques mediterráneos. En ella hay registros sobre nanofanerófitos (Olea europea) y principalmente sobre mesofanerófitos de vegetación mediterránea, y especialmente en encinares (Quercus ilex), pero también sobre quejigo ( $Q$. faginea), alcornoques $(Q$. suber), maquia o en campos frutales, también sobre otros variados sustratos (Pinus, Phillirea, Crataegus, Rosa, Cytisus, Juniperus, Tamarix, hierba) en estos medios térmicos. Su distribución altitudinal global comprende entre los 200-1.800 m, en la península se han hallado ejemplares entre 300-1.476 m. Las densidades de poblaciones de imagos siempre son bajas. Existen citas de parasitoides de la familia Ichneumonidae: Nemeritis caudata de Sierra Nevada y Alicante, Nemeritis specularis de Alicante y de un hiperparásito (Chalcidoidea: Perilampidae): Perilampus polypori de Alicante (H. Aspöck et al., 1991).

FENOLOGÍA: Presenta una extensa distribución anual en la aparición de imagos, dada su amplia versatilidad ecológica dependiendo de la latitud y altitud. La actividad de los imagos es monovoltina, estacionaria transcurriendo durante los meses de V-VI, en la península existen registros en los meses IV-VII, con citas puntuales en III.

\section{VARIABILIDAD: Insignificante.}

ESTADIOS PREIMAGINALES: Las larvas son exclusivamente corticícolas. Hay un número elevado de registros de larvas en Quercus, Amigdalus y Pinus. Interesante el dato ahora citado en agalla de quejigo. Se han citado desarrollos larvarios como mínimo dos o tres años, en algunos casos hasta 4 años. La última 
fase de hibernación transcurre en fase de larva, por tanto es un ciclo vital de tipo I (fig. 18). El estadio de pupa se mantiene entre 9-21 días.

Existen citas de parasitoides de la familia Ichneumonidae: Nemeritis caudata de Sierra Nevada y Alicante, Nemeritis specularis de Alicante y de un hiperparásito (Chalcidoidea: Perilampidae): Perilampus polypori de Alicante (H. Aspöck et al., 1991). Morfología y aspecto de la larva en figura 145 y en H. Aspöck et al. (1991).

DISTRIBUCIÓN: Citado de España y Portugal (fig. 158). Elemento faunístico atlantomediterráneo, monocéntrico con una expansividad muy escasa. Se sospecha que su distribución se limita exclusivamente a la Península Ibérica.

DISCUSIÓN: Entre los rafidiópteros de la Península Ibérica, Subilla aliena ocupa por su morfología una posición aislada, y no se puede confundir con ninguna otra especie. Esta íntimamente emparentada con la especie no ibérica Subilla confinis, presentando ciertas diferencias en la genitalia del $\widehat{\partial}$.

Mediante un estudio superficial puede presentar un parecido con Xanthostigma aloysianum, pero cuyo pterostigma es más corto y la parte basal de la Ma en el ala posterior presenta un recorrido transversal (figs. 26, 27). Esta especie ha sido citada junto a Atlantoraphidia maculicollis, Harraphidia laufferi, Hispanoraphidia castellana, Ohmella baetica, Ohmella bolivari, Ohmella postulata, Ohmella libidinosa, Ohmella casta y Fibla (Fibla) hesperica.

\section{Género Xanthostigma Navás, 1909}

Xanthostigma Navás, 1909b: 3 (d, a), [especie tipo por tautonimia: Raphidia xanthostigma Schummel, 1832].

Considerado como sección de Raphidia Linnaeus, 1758: Navás, 1909b: 3 (a, d).

Considerado como subgénero de Raphidia Linnaeus, 1758: H. Aspöck \& U. Aspöck, 1973a: 52 (tx). U. Aspöck, 1974 (tx). H. Aspöck et al., 1975: 373 (1). U. Aspöck, 1975: 542 (tx). Popov et al., 1978 (dis). H. Aspöck et al., 1980a: vol. I: 86 (lst), 87 (dis), 94 (a), 122 (tx), vol. II: 256, 257 (dis).

Considerado como género: H. Aspöck, 1986: 23 (tx), 25 (dis). H. Aspöck, 1987: 156 (dis). H. Aspöck et al., 1989: 88, 102 (dis). U. Aspöck \& H. Aspöck, 1990: 99, 100 (tx), 100 (dis). H. Aspöck et al., 1991: vol. I: 76 (fe), 111 (lst), 113, 294, 296 (tx), 114, 295, 296, 558, 564 (dis), 294 (a, mf), 295 (bio, 1, p, pa, ô, +), vol. II: 419, 420 (dis). Oswald \& Penny, 1991: 66 (lst). H. Aspöck \& Hölzel, 1996: 38 (dis). H. Aspöck et al., 2001: 20, 41 (tx), 41 (dis). H. Aspöck, 2002: 38, 44, 45 (1), 44, 45 (pa). H. Aspöck \& U. Aspöck, 2007: 100 (rf), 103 (dis). Monserrat \& Papenberg, 2006: 204 (rf). Letardi \& Pantaleoni, 2007: 89 (1st). Monserrat \& Papenberg, 2010: 48, 63 (rf). Haring et al., 2011: 11, 12 (tx), fig.S4 (dis). U. Aspöck et al., 2012: 577 (lst), 579 (f). Monserrat \& Papenberg, 2012: 292 (rf). H. Aspöck \& U. Aspöck, 2013: 12 (tx), 43 (gen).

= Raphidilla Navás, 1915d: 25 (d, a), [especie tipo por designación original: Raphidia xanthostigma Schummel, 1832].

Navás, 1919: 6 (dis, 1st). Banks, 1923: 115 (a, tx). Navás, 1925: 46, 47 (tx). Lestage, 1928: 32 (c), 30, 31 (tx). Navás, 1928a: 19 (a). Navás, 1934b: 30 (a), 30-32 tx). Carpenter, 1936: 93 (tx).
Principi, 1952: 3 (tx). Steinmann, 1963: 184, 186 (c). Tjeder, 1964: 109 (rf). Steinmann, 1967 (tx). H. Aspöck \& U. Aspöck, 1967: 225 (tx). Oswald \& Penny, 1991: 66 (lst).

Considerado como subgénero de Raphidia Linnaeus, 1758: H. Aspöck \& U. Aspöck, 1968b: 63 (tx). H. Aspöck et al., 1969: 156 (lst). H. Aspöck \& U. Aspöck, 1973a: 52 (sin).

Xanthostigma es un género muy bien diferenciado, marcado por su venación alar y los segmentos genitales del $\hat{\partial}$ y de la $q$. Engloba cinco especies conocidas de Europa (excepto el sur de la península de los Balcanes), y Asia (Caúcaso, Siberia hasta Kamatschka, Kasachstan, Mongolia y el norte de China), de las cuales Xanthostigma aloysianum y Xanthostigma corsicum están citadas en la Península Ibérica.

Xanthostigma aloysianum (Costa, 1855)

Raphidia aloysiana Costa, 1855: Costa, 1855: 4 (d). Albarda, 1891: 68 (rf). Esben-Petersen, 1913a: 6 (a, dis, 1st), 15 (a). Navás, 1913b: 11 (dis). Condé \& Pagès, 1959: 193 (1st). H. Aspöck \& U. Aspöck, 1969b: 34 (rf). Principi, 1980: 106 (dis). Monserrat \& Triviño, 2013: 15 (lst).

Raphidilla aloysiana (Costa, 1855): Navás, 1915d: 25 (tx). Navás, 1919: 50 (c), 55, 56 (a, dis, mf). Navás, 1928b: 77 (dis), 78 (mf, đ̊), 79 (rf). Navás, 1934b: 31 (c), 34, 35 (mf), 35 (a, dis, đ̂, ㅇ), Principi, 1952: 7 (rf). Steinmann, 1964: 215 (c). Monserrat \& Triviño, 2013: 15 (lst).

Agulla aloysiana (Costa, 1855): Principi, 1952: 6 (a, tx), 7 (ô, mf). Principi, 1960: 329, 330 (rf), 331, 332 (ठ̋). Principi, 1961: 99 (ㅇ), 99, 100 (a, mf, tx), 99, 101-104 (ठ̋). Bartoš, 1965a: 90, 93 (rf). Bartoš, 1965b: 466 (rf). Principi, 1966: 367 (mf), 367, 369 (rf), 371 (dis). Bartoš, 1967: 325 (rf). H. Aspöck \& U. Aspöck, 1969a: 25 (dis, tx), 50 (rf), 62 (ठ̋). Zelený, 1969: 36 (dis). Monserrat \& Triviño, 2013: 15 (1st).

Raphidia (Agulla) aloysiana Costa, 1855: Eglin-Dederding, 1967: 206 (dis). Monserrat \& Triviño, 2013: 15 (lst).

Raphidia (Raphidilla) aloysiana Costa, 1855: Condé \& Pagès, 1956a: 127, 129 (a), 129 (dis). H. Aspöck \& U. Aspöck, 1968b: 63 (tx). H. Aspöck \& U. Aspöck, 1971a: 9 (dis). Monserrat \& Triviño, 2013: 15 (lst).

Raphidia (Xanthostigma) aloysiana Costa, 1855: H. Aspöck et al., 1974a: 46 (rf), 46, 48, 49 (dis), 48 (1). H. Aspöck et al., 1974c: 2 (lst), 4, 14 (l), 14 (dis), 18 (bio), 19 (gen). H. Aspöck, 1977: 17, 18 (rf). Popov et al., 1978 (rf). Eglin-Dederding, 1979: 494 (dis). H. Aspöck et al., 1980a: vol. I: 37 (c), 114, 123 (a), 123 (bio, tx), 123, 352 (dis), vol. II: 38 (ठ)), 40 (mf), 167 (a), 256 (dis). Gepp, 1986a: 138 (l). Monserrat, 1986a: 96 (dis). Monserrat \& Triviño, 2013: 15 (lst).

Xanthostigma aloysiana (Costa, 1855): H. Aspöck et al., 1991: vol. I: 88, 280, 295, 302 (bio), 280, 300, 301, 349 (a), 125, $126,129,131,132$ (c) $, 125,129,131,132,280,302,548$, 550, 551, 574, 575 (dis), 280, 295 (†), 280, 295, 301 (さ), 294, 301 (tx), 301 (mf), 614 (1st), vol. II: 51 (mf), 71 (a), 146 (ठ), 472 (dis). Poivre, 1991 (dis). Rausch \& H. Aspöck, 1992: 35 (lst). Albertina et al., 1995: 5 (dis). Marín \& Monserrat, 1995: 111 (dis, gen, 1st). Monserrat, 1996: 12 (1st). H. Aspöck \& Hölzel, 1996: 46 (dis). Letardi \& Pantaleoni, 1996: 280 (dis). H. Aspöck et al., 1998b: 126 (dis, tx). Pantaleoni \& Letardi, 1998: 18 (dis). H. Aspöck et al., 2001: 42 (tx), 42, 330, 336 (dis). Rausch et al., 2004: 164 (rf). Pantaleoni, 2005: 76 (lst). H. Aspöck \& U. Aspöck, 2007: 98, 103, 104 (dis), 102 (rf). Monserrat \& Papenberg, 2010: 63 (rf), 63, 64 (dis). Haring et al., 2011: 6 (dis, 1st), 10 (tx), fig.S2 (f). Monserrat \& Papenberg, 2012: 292 (rf). H. Aspöck \& 
U. Aspöck, 2013: 33 (lst), 44 (gen), 81 (eco). Monserrat \& Triviño, 2013: 15, 45 (dis, 1st, tx).

= Raphidilla puella Navás, 1915: Navás, 1915c: 198 (d, đ̋), 198, 199, (a, mf). Navás, 1915a: 792 (1st). Navás, 1915b: 873 (dis). Bohigas \& Sanchez, 1917: 319 (lst). Navás, 1919: 50 (c), 57, 58 (a, mf), 58 (dis). Navás, 1923: 70 (c), 72 (dis). Navás, 1928a: 20 (c), 25 (ð), 25, 26 (a), 26 (dis, mf). Lestage, 1928: 31 (a). Navás, 1931: 31 (dis). Lacroix, 1933: 152 (rf, ㅇ). Navás, 1933a: 121 (lst). Navás, 1934a: 6 (dis). Navás, 1934b: 31 (c), 37 (a, dis, mf, ô, o ). Steinmann, 1964: 214 (a), 215 (c). Bartoš, 1965a: 96 (a, đ̊, tx). H. Aspöck et al., 1980a: 123 (sin). Monserrat \& Triviño, 2013: 15 (lst).

= Agulla puella (Navás, 1915): Bartoš, 1965b: 466 (rf). Monserrat \& Triviño, 2013: 15 (lst).

= Raphidilla soror Navás, 1915: Navás, 1915c: 199 (d), 199, 200 (mf), 200 (a, §̂). Navás, 1915a: 792 (1st). Navás, 1915b: 860 (a, + , mf), 860, 873 (dis). Navás, 1919: 50 (c), 58, 59 (a, mf), 59 (dis). Navás, 1923: 71 (c), 72 (dis). Navás, 1928a: 21 (c), 26, 27 (mf), 27 (a, dis). Navás, 1933a: 121 (1st). Navás, 1934a: 6 (lst). Navás, 1934b: 31(c), 38 (a, dis, mf). Steinmann, 1964: 214 (a), 215 c). Bartoš, 1965a: 96 (a, tx). H. Aspöck et al., 1980a: 123 (sin). Monserrat \& Triviño, 2013: 15 (1st).

= Agulla soror (Navás, 1915): Bartoš, 1965b: 466 (a). Monserrat \& Triviño, 2013: 15 (lst).

TIPO: holotipo $q$ (Istituto e Museo di Zoologia della Univerità di Napoli).

\section{LOCALIDAD TÍPICA: Abruzzi (Italia).}

MATERIAL ESTUDIADO: ESPAÑA: Granada: Fuente de la Cortichuela, 30SVG5904, 1.900 m, 3.VII.1986, 2 우 우 sobre Pinus sylvestris, V. J. Monserrat (VM). Orense: Osera, 29TNH8610, 650 m, 4.VI.1977, 1 ○ (a*, $\odot *)$, V. J. Monserrat (VM). Zaragoza: Alto del Moncayo, 30TWM9728, 1.750 m, 3.VI.1994, 1 ㅇ, sobre Salix sp., F. Marín (VM), Biel, 30TXM6995, 780 m, 15.VI.1931, 1 ㅇ, leg.? (MNHN), 17.VI.1931, 1 ô, leg.? (MNHN).

MORFOLOGÍA EXTERNA: Es una especie de tamaño pequeño. El clípeo y el labro tienen una coloración entre amarillenta y ocre. El flagelo en su parte basal es ocre-amarillo y en la apical pardo. El pronoto es pardo apareciendo zonas más claras en su superficie, con un borde ocre ancho. Respecto a los apéndices locomotores, las coxas II y III son pardas, el fémur III es amarillo, y en algunos casos presenta bandas pardas longitudinales. Los demás artejos son amarillos.

La longitud del ala anterior es de 7-8 $\mathrm{mm}$ en los

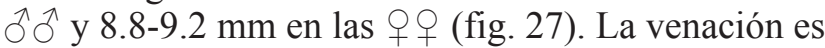
mayoritariamente de un tono pardo claro. La costa, el radio, las venas de la base del ala anterior y del tercio proximal del ala posterior son amarillos. Entre el radio y el sector radial del ala anterior, junto al radio, hay dos celdillas (fig. 27). En el abdomen los terguitos son pardos con una banda amarilla.

SEGMENTOS GENITALES: En los $\widehat{\partial} \widehat{\partial}$ el apodema del estilo adquiere una posición oblicua (fig. 49). El proceso del gonocoxito está fuertemente esclerotizado, es puntiagudo, en forma de gancho, orientado en dirección lateral y quedando su ápice oculto bajo los gonocoxitos. Los estilos son muy largos y presentan una bifurcación en el extremo (figs. 49, 50). La base de la hipovalva es fina con una incisión central ancha (fig. 50). No existen parámeros. El gonarco es indetectable. El hipandrio interno es delicado y claro (fig. 82).

En las 90 el intersegmento $\mathrm{S} 7 / 8$ se encuentra esclerotizado presentando unos salientes característicos en posición anterior (fig. 105). Respecto a la genitalia interna, el atrio está doblado y es compacto (fig. 127). El sáculo es corto y está muy doblado. El receptáculo seminal presenta unas glándulas filiformes que poseen cada una un apéndice globular. Estos apéndices globulares no han sido observados en ningún ejemplar de la Península Ibérica (fig. 127).

BIOLOGÍA: Existen pocos datos sobre la biología de Xanthostigma aloysianum. Hay citas de adultos en sotobosques de clima templado, sobre todo de pinares abiertos con maquia en altitudes comprendidas entre los 600-1.900 m, en la península entre los 650-1.900 m. El número de individuos es siempre bajo, siendo en la mayoría de los casos individuos aislados.

FENOLOGÍA: La actividad de los imagos es monovoltina y estacionaria transcurriendo entre los meses de V-VII. Las citas españolas son de los meses VI y VII.

VARIABILIDAD: Tanto en los $\widehat{\partial} \hat{0}$ como en las + 우 la variabilidad es escasa. Las poblaciones italianas y españolas coinciden morfológicamente. Existe un ejemplar de la Península Ibérica de Osera (Orense) sin lóbulos en las glándulas del receptáculo seminal.

ESTADIOS PREIMAGINALES: La banda dorsal es muy fina y la banda lateral se continúa a través de los segmentos sin presentar apenas puentes pigmentarios (fig. 146). Se desconoce con exactitud cual es el tipo de sustrato sobre el que se desarrolla la larva. Se han capturado individuos en la provincia de Barcelona, en una zona carente de vegetación arbórea, lo que hace pensar que la larva debería de ser de vida edáfica (entre la hojarasca), a lo que se suma que no existen registros de larvas bajo las cortezas (H. Aspöck et al., 1974c). El tiempo de desarrollo es desconocido. En otras especies del género ( $X$. xathostigma y $X$. corsicum) se han citado desarrollos larvarios de una media de 2 años, en donde la última hibernación transcurre en fase de larva y por tanto son ciclos de tipo I (fig. 18), y el estadio de pupa se mantiene entre 10-25 días. Morfología y aspecto de la larva en figura 146 y en H. Aspöck et al. (1991).

DISTRIBUCIÓN: Europa: Italia, sur de Suiza, sur de Francia, norte de España (fig. 159). Elemento faunístico monocéntrico, adriatomediterráneo de escasa capacidad de expansión. En la Península Ibérica tiene aparentemente una distribución exclusivamente septentrional. La cita de Fuente de la Cortichuela (Granada) dentro del material estudiado presenta muchas dudas. Se trata de dos ejemplares 0 c cuya venación alar coincide plenamente con Xanthostigma 
aloysianum, pero la genitalia se encontraba en muy mal estado y por tanto su identificación exacta no ha sido posible (fig. 159).

DISCUSIÓN: Xanthostigma aloysianum es la especie más emparentada con $X$. corsicum y $X$. xanthostigma, pero presenta diferencias morfológicas. En los $\hat{\delta} \hat{o}$ de $X$. aloysianum existen en el ala anterior dos celdillas entre el radio y su sector junto al radio (fig. 27), al igual que en $X$. xanthostigma, pero no en $X$. corsicum, donde hay tres (fig. 28). Es de suponer que la cita de Navás (1905d: 18) de Cataluña (Barcelona, Montserrat) considerada por el autor como Raphidia xanthostigma Schummel, se trate en realidad de $X$. aloysianum por su semejanza morfológica y por concordancia de la zona de distribución. Respecto a la genitalia del $\widehat{\partial}$ existen claras diferencias en la forma del ectoprocto que en $X$. aloysianum se encuentra menos prolongado en dirección caudal que en las otras dos especies (figs. 49, 51). A su vez, la bifurcación existente en el extremo de los estilos es exclusiva de $X$. aloysianum (figs. 49, 50), así como los procesos de los gonocoxitos que en $X$. aloysianum tienen una orientación lateral, en $X$. corsicum su orientación es posterior (figs. 50, 52), siendo inexistentes en $X$. xanthostigma. La forma de la costilla central de la hipovalva, en el caso de $X$. aloysianum, presenta los brazos más abiertos que en las otras dos especies (figs. 50, 52). Respecto a los parámeros, no existen en $X$. aloysianum y sí en las otras dos especies. En las 우 (figs. 105, 106), sobre todo varía el número de apéndices globulares presentes en las glándulas del receptáculo seminal, que en $X$. aloysianum son uno o ninguno, mientras que en $X$. xanthostigma y $X$. corsicum son de dos a tres (figs. 127, 128).

\section{Xanthostigma corsicum (Hagen, 1867)}

Raphidia corsica Hagen, 1867: Hagen, 1867: 496 (d, mf), 496, 497 (). Brauer, 1876: 288 (dis). Rostock, 1888 (1st). Albarda, 1891: 69 (rf). Esben-Petersen, 1913a: 7 (lst). Banks, 1923: 116 (mf). Kimmins, 1930: 187 (lst). Mosely, 1932: 182 (lst). Tjeder, 1964: 108 (rf). Monserrat \& Triviño, 2013: 15 (lst).

Raphidilla corsica (Hagen, 1867): Navás, 1919: 69 (a). Steinmann, 1964: 217 (ð̂, o). Monserrat \& Triviño, 2013: 15 (lst).

Raphidia (Xanthostigma) corsica Hagen, 1867: Popov et al., 1978 (rf). H. Aspöck et al., 1980a: vol. I: 37 (c), 124 (a, bio, ô, tx), 124, 352 (dis), vol. II: 39 (đ) ), 167 (a), 257 (dis). Alrouechdi, 1982 (bio). Séméria, 1984: 178 (bio, dis). Pantaleoni, 1986: 169 (dis). Monserrat \& Triviño, 2013: 15 (lst).

Xanthostigma corsica (Hagen, 1867): Pantaleoni, 1990a: 76 (dis). H. Aspöck et al., 1991: vol. I: 88, 295, 300 (bio), 129, 131, 132, 134, 548, 550, 551, 554, 574, 575, 610 (dis), 129, 132, 134 (c), 131, 614 (lst), 147 (ふ) , 295, 300 (1, ふૈ, 우), 294, 299, 300 (tx), 298-300, 349 (a), 299, 300 (mf), vol. II: 51 (mf), 71 (a), 343, 352, 369, 370 (1), 470, 471 (dis). Poivre, 1991 (dis). Pantaleoni, 1994: 209 (dis). Albertina et al., 1995: 5 (dis). H. Aspöck \& Hölzel, 1996: 43 (dis). Letardi \& Pantaleoni, 1996: 279 (dis). H. Aspöck et al., 1998b: 126 (dis, tx). Letardi, 1998: 148 (rf). Pantaleoni \& Letardi, 1998: 18 (dis). H. Aspöck et al., 1999: 71 (1). H. Aspöck et al., 2001: 42 (tx), 42, 330, 336 (dis). Letardi \& Maltzeff, 2003 (dis, rf). Rausch et al., 2004: 161 (a). H. Aspöck
\& U. Aspöck, 2007: 98, 103, 104 (dis), 102 (rf). Monserrat \& Papenberg, 2010: 63 (rf), 63, 64 (dis). Haring et al., 2011: 6 (dis, 1st), 10 (tx), fig.S2 (f). Monserrat \& Papenberg, 2012: 292 (rf). H. Aspöck \& U. Aspöck, 2013: 33 (1st), 55 (dis, , 1), 77 (gen), 88 (eco). Monserrat \& Triviño, 2013: 15, 45 (dis, lst, tx).

$=$ Raphidia insularis Albarda, 1891: Albarda, 1891: 117 (d), 117119 (dis, mf). Weele, 1910: 87 (lst). Navás, 1912: 36 (dis). Esben-Petersen, 1913a: 5 (a), 7 (dis). Esben-Petersen, 1913b: 26 (dis). Banks, 1923: 115 (a), 116 (tx). Kimmins, 1930: 187 (dis, 1st). Mosely, 1932: 182 (1st). H. Aspöck et al., 1980a: 124 (sin). Pantaleoni, 1986: 169 (sin).

= Puncha insularis (Albarda, 1891): Navás, 1915a: 796 (dis). Navás, 1915e: 27 (tx). Navás, 1915f: 2 (a, dis, mf, ㅇ). Navás, 1919: 24 (c), 25 (a, mf, đ̂, ㅇ). Navás, 1933a: 120 (1st). Navás, 1934a: 4 (dis). Navás, 1934b: 16 (c), 17, 18 (mf), 18 (a, dis, đ̂, ㅇ).

= Raphidia (Puncha) insularis Albarda, 1891: Condé \& Pagès, 1956a: 129 (dis). Berland, 1962: 21 (lst, mf). Séméria \& Berland, 1988: 36 (mf).

= Agulla insularis (Albarda, 1891): H. Aspöck \& U. Aspöck, 1966b: 127 (mf). Principi, 1966: 365, 366, 369 (a), 365, 369 (dis), 367 (mf, tx). Capra, 1976: 547, 556 (dis).

= Raphidia (Raphidilla) insularis Albarda, 1891: H. Aspöck \& U. Aspöck, 1968b: 63 (tx). Kusdas, 1972: 161 (dis).

= Puncha italica Navás, 1927: Navás, 1927: 9 (d, đ̊), 10 (a, mf). Navás, 1933a: 120 (1st). Navás, 1934a: 4 (dis). Navás, 1934b: 8, 19 (mf, đ)), 16 (c), 19 (a, dis). Castellani, 1957: 9 (rf). H. Aspöck et al., 1980a: 124 (sin).

= Agulla italica (Navás, 1927): Principi, 1966: 366, 369 (a), 369 (tx), 369, 371 (dis), 370 (ふ).

= Raphidia (Raphidilla) italica (Navás, 1927): H. Aspöck \& U. Aspöck, 1968b: 63 (tx).

Raphidia xanthostigma Schummel, 1832: Schneider, 1843: 71 (1, mf, mon). Brauer \& Löw, 1857: 53 (1st). Dziedzielewicz, 1891: 94 (rf). Navás, 1905a: 38 (dis, lst, mf) - ie. Navás, 1908: 131 (c), 132 (dis, mf) - ie. Navás, 1909a: 378 (dis) - ie. Navás, 1911b: 36 (c, mf) - ie. Navás, 1913a: 86 (dis) - ie. Navás, 1913c: 107 (lst) - ie. Monserrat \& Triviño, 2013: 15 (lst).

Raphidilla xanthostigma (Schummel, 1832): Navás, 1915b: 874 (rf). Navás, 1928a: 23 (a, dis, mf) - ie. Navás, 1928b: 79 (dis) ie. Tjeder, 1937b: 121 (rf). Monserrat \& Triviño, 2013: 15 (lst). Raphidia cognata Rambur, 1842: Hagen, 1864 (1st) - ie.

TIPO: Lectotipo (solo queda la cabeza, pronoto, fragmentos del tórax y dos apéndices locomotores (Museum of Comparative Zoology, Cambridge (Mass.).

\section{LOCALIDAD TÍPICA: Córcega (Francia).}

MATERIAL ESTUDIADO: ESPAÑA: Ávila: Puerto de Castilla, 30TTK7663, $1.171 \mathrm{~m}, 8$. VII.1983, 1 을 en vegetación de galería de río, C. Urones (VM), Puerto de la Hoya, 30TTK7277, $1260 \mathrm{~m}, 10$. VI.2006, $1 \mathrm{\delta}$, M. Portillo (USAL). Salamanca: Candelario, 30TTK6772, 1.200 m, 14.V.1982, 1 ô, 9.VI.1982, 1 , C. Urones (VM).

De la especie Xanthostigma corsicum existen muy pocas citas en la Península Ibérica, y se aprecian claras diferencias morfológicas intraespecíficas con respecto a los individuos europeos.

MORFOLOGÍA EXTERNA: Descripción del $\sigma^{\Uparrow}$ de la Península Ibérica: Xanthostigma corsicum es una especie de tamaño pequeña. El clípeo es amarillo y el labro amarillo-ocre. El escapo y el pedicelo son amarillos. El flagelo, en su región basal, es amarillo-ocre, 
siendo el resto pardo. El pronoto es delgado, pardo claro. Respecto a los apéndices locomotores, las coxas II y III son pardas, el trocánter III y el fémur III presentan zonas pardas. Los artejos restantes son amarillos.

La longitud del ala anterior es de $6.5-8 \mathrm{~mm}$ en los

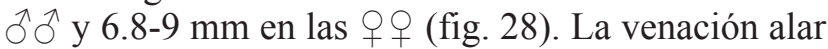
en general es parda. La costa y el radio son pardos en parte o totalmente. Las venas de la base del ala anterior así como las del tercio basal del ala posterior son amarillentas. Entre el radio y el sector radial, junto al radio, hay tres celdillas (fig. 28). En el abdomen los terguitos son pardos oscuros con una banda central amarilla-ocre y en su parte caudal existe una mancha lateral amarilla. Los esternitos son pardo oscuro con el borde caudal amarillo.

SEGMENTOS GENITALES: En los $\widehat{\jmath} \widehat{\jmath}$ el apodema del estilo adquiere una posición oblicua (fig. 51). El proceso del gonocoxito está fuertemente esclerotizado y puntiagudo, está orientado en dirección caudal (figs. 51, 52). Los estilos no son tan largos como en Xanthostigma aloysianum (figs. 49-52), y tienen el extremo afilado (figs. 51, 52). La hipovalva es impar y su costilla central esclerotizada es corta y fina (fig. 52). Existen zonas esclerotizadas laterales pares, en forma de gancho y que han sido consideradas en ocasiones como parámeros, pero que probablemente pertenezcan a la hipovalva (fig. 52). Los parámeros son impares, difíciles de observar y están reducidos. El gonarco es par, alargado en forma de horquilla (figs. 51, 52). El hipandrio interno es pequeño (fig. 83). El ectoprocto es grande con la región caudal ancha (fig. 51).

En las 99 el intersegmento $\mathrm{S} 7 / 8$ está débilmente esclerotizado (fig. 106). El $8^{\circ}$ terguito es delgado y a la altura del espiráculo está abombado (fig. 106). Respecto a la genitalia interna, el sáculo presenta una estriación longitudinal (fig. 128). El receptáculo seminal es piriforme con glándulas filiformes que poseen cada una, de dos a tres apéndices globulares (fig. 128).

BIOLOGÍA: Hay numerosas citas de imagos sobre todo en vegetación arbustiva, frecuentemente en Genista sp. Aparecen en biotopos cálidos de Quercus y Pinus con abundante sotobosque. Las dos citas de España no ofrecen mucha información respecto a su biología, pero por su situación parece adaptada a biotopos con cierta humedad (citamos un ejemplar en vegetación de galería de río). Su distribución vertical comprende desde el nivel del mar hasta los $1.700 \mathrm{~m}$, en la península se conocen datos entre 1.171-1.260 m. Normalmente las densidades de población de los individuos de Italia y Francia suelen ser altas, no parece ocurrir así en la Península Ibérica.

FENOLOGÍA: La actividad de los imagos es monovoltina y estacionaria transcurriendo entre los meses de V-VII, tanto en las poblaciones de la Península Ibérica como en el resto de Europa.
VARIABILIDAD: En base a la información existente, los individuos de la Italia continental son de media más grandes, siendo el ala anterior $1 \mathrm{~mm}$ más grande que los insulares. El pterostigma también es más largo, sobrepasando en dirección proximal la $1^{\mathrm{a}}$ celdilla postigmal. Esta celdilla suele ser más larga que la de los individuos citados en Córcega. La población de Elba se diferencia por tener un pterostigma más pequeño y una hipovalva más corta. En ejemplares de Sicilia el ala anterior es más larga (unos $0,5 \mathrm{~mm}$ ) y el pronoto algo más claro. La hipovalva de los individuos de Italia continental es indudablemente más larga (fig. 54). La base de la horquilla de la hipovalva es más estrecha en las poblaciones de Sicilia e Italia penínsular que en la población de Córcega (figs. 53-56). El ổ de Francia continental llama la atención por su labro de un amarillo intenso, con manchas pardas muy contrastadas sobre el clípeo pero coinci-

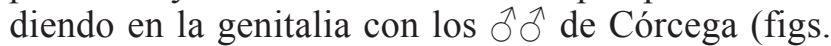
55, 56). El $\sigma^{\lambda}$ de España se caracteriza por tener la base de la varilla de la hipovalva mucho más ensanchada que las poblaciones de Italia (figs. 51-54). La forma del estilo del $\sigma^{\Uparrow}$ de España también varía algo, siendo algo menos curvado y su ápice menos romo que en los individuos italianos y corsos (figs. 51-56). Todo esto sugiere un evidente proceso de especiación aún por dilucidar.

ESTADIOS PREIMAGINALES: La banda dorsal es relativamente ancha (fig. 147). Las bandas laterales son anchas y la figura dorsal se caracteriza por un saliente a modo de espina (fig. 147). La figura ventral ocupa la parte media del segmento, y presenta una clara banda ancha media ventral. Las larvas son probablemente edáficas. Existen algunos registros de larvas encontradas bajo cortezas de Pinus y Quercus. Se han citado desarrollos larvarios de una media de 2 años, en donde la última hibernación transcurre en fase de larva y por tanto son ciclos de tipo I (fig. 18). Mediante cría ex ovo se ha confirmado un periodo de desarrollo de dos años. El estadio de pupa (en laboratorio) es de entre 13-25 días. Morfología y aspecto de la larva en figura $147 \mathrm{y}$ en H. Aspöck et al. (1991).

DISTRIBUCIÓN: Europa: Italia (islas de Córcega, Cerdeña, Sicilia, Elba, e Italia continental), Francia y España (fig. 160). Se considera a Xanthostigma corsicum como un elemento faunístico policéntrico tirrénicoadriato-atlanomediterráneo. Sobre el continente su distribución está muy fragmentada, dando imagen de una especie politípica (H. Aspöck et al., 2001).

DISCUSIÓN: Xanthostigma corsicum se diferencia de las especies con las que guarda una mayor relación de parentesco (Xanthostigma xanthostigma y Xanthostigma aloysianum) de forma clara, y por tanto no se plantea un problema taxonómico. Existe un polimorfismo intra-específico al presentar variaciones en la morfología de la genitalia del $\widehat{\partial}$ según 
la procedencia del individuo (figs. 49-56), hecho que sugiere un marcado proceso de especiación en marcha.

Género Atlantoraphidia H. Aspöck \& U. Aspöck, 1968 Atlantoraphidia H. Aspöck \& U. Aspöck, 1968b: 60, (d, ぶ), 61 (q, mf, tx), [especie tipo por designación original: Raphidia maculicollis Stephens, 1836].

Considerado como subgénero de Raphidia Linnaeus, 1758: H. Aspöck \& U. Aspöck, 1968b: 60 (d, ठ)), 61 (, mf, tx). H. Aspöck et al., 1975: 371 (1). H. Aspöck et al., 1980a: vol. I: 86, 87 (dis), 116 (tx), vol. II: 252 (dis).

Considerado como género: H. Aspöck, 1986: 25, 26 (dis). H. Aspöck et al., 1989: 89 (dis). H. Aspöck, 1990: 53 (tx), 54 (dis). H. Aspöck et al., 1991: vol. I: 76, 348 (bio), 111, 115, 347, 348 (tx), 347 (a, đ) , 348 (ㅇ, 1), 348, 555, 559 (dis). vol. II: 423 (dis). Oswald \& Penny, 1991: 63 (1st). U. Aspöck \& H. Aspöck, 1994: 39 (tx). H. Aspöck \& Hölzel, 1996: 38 (dis). H. Aspöck, 1998b: 39 (tx). H. Aspöck et al., 2001: 19, 50 (tx), 50 (dis). H. Aspöck, 2002: 38 (1), 41 (p). H. Aspöck \& U. Aspöck, 2007: 99 (dis). Monserrat \& Papenberg, 2010: 48 (rf). Haring et al., 2011: 12 (tx), fig.S3 (dis). U. Aspöck et al., 2012: 577 (lst), 579, 580 (f). Monserrat \& Papenberg, 2012: 292 (rf). H. Aspöck \& U. Aspöck, 2013: 12 (tx), 38 (gen).

Atlantoraphidia es un género monotípico cuya única especie es Atlantoraphidia maculicollis, citada de Europa occidental y central, incluyendo la Península Ibérica (fig. 161).

\section{Atlantoraphidia maculicollis (Stephens, 1836)}

Raphidia maculicollis Stephens, 1836: Curtis, 1824: 37 (1st, rf). Stephens, 1829 (1st). Stephens, 1836: 131 (d). Curtis, 1837 (lst). Walker, 1853: 212 (a, dis, mf). McLachlan, 1868: 160, 161, (mf), 161 (a, dis), 162 (tx). McLachlan, 1870: 18 (lst). Brauer, 1876: 288 (dis). Selys-Longchamps, 1888: 161 (dis). Albarda, 1889 (sin, dis). McLachlan, 1890: 304 (a). Albarda, 1891: 86, 89 (a), 72, 73, 75 (rf). McLachlan, 1894: 186 (dis, 1). Morley \& Elliott, 1895: 193, 194 (fe). Lucas, 1901 (dis). McLachlan, 1902: 129 (dis). Navás, 1904: 143 (1st). Navás, 1905a: 37 (a, bio, mf). Navás, 1905b: 507 (dis, mf, ㅇ). Navás, 1905c: 125 (dis, lst). Weele, 1907 (dis). Navás, 1908: 35 (c), 35, 43 (a), 36 (dis). Lucas, 1909a (bio, dis). Lucas, 1909b (dis). Lucas, 1909c (dis). Morley, 1909: 142 (a, dis). Navás, 1909a: 378 (dis). Navás, 1909b: 4 (dis, ie). Porritt, 1909: 183 (dis, lst). Weele, 1910: 88 (1st). Navás, 1911b: 36 (c, mf). Esben-Petersen, 1913a: 7 (dis, 1st). Lacroix, 1913: 20 (c). Lucas, 1913 (dis). Lyle, 1913: 188 (fe), 187, 188 (h), 187 (la, pa). Navás, 1913c: 107 (1st). Williams, 1913: 6, 7 (bio), 7 (h, 1), 7, 8 (pa). Lucas, 1914a (dis). Navás, 1914a: 41 (dis). Navás, 1914b: 35 (dis). Navás, 1914c: 35 (dis, ㅇ). Campion, 1915: 24 (1), 25, 26 (fe). Lucas, 1915: 180 (1). Navás, 1915a: 785 (rf), 787, 794 (dis). Navás, 1915b (c, mf). Navás, 1915g: 331 (dis, ie). Mac Gillavry, 1916 (dis). Lucas, 1917: 87 (dis, mf). Tillyard, 1919: 697 (mf). Lucas, 1920 (dis). Haines, 1922: 84 (dis). Lucas, 1922: 287 (rf). Lucas, 1923: 219 (dis, pa), 219, 229 (1, mf). Withycombe, 1923: 33, 34 (p), 33-35 (a). Lucas, 1924: 177 (dis). Lucas, 1925: 155 (dis). Killington, 1926: 110 (dis). Lucas, 1926: 290 (dis). Killington, 1927: 40 (dis, p). Lucas, 1927a: 169 (dis). Lucas, 1927b: 7 (1st). Stitz, 1927a: 2 (a), 4 (c). Killington, 1928: 50 (dis). Lucas, 1928a: 217 (bio), 217, 218 (dis). Lucas, 1928b: 34-41 (bio, dis, p, tx). Killington, 1929: 5 (p), 5, 7 (a), 6 (c), 7 (dis, mf). Lucas, 1930: 269 (dis). Esben-Petersen, 1931: 6 (dis, ie). Killington, 1931: 73 (1, p). Lucas, 1931: 243 (dis). Mikulski, 1931: 90 (dis). Killington, 1932a: 8 (dis). Killington, 1932b: 59 (bio), 64 (dis), 61 (fe). Kimmins, 1933: 88 (dis). Kimmins, 1934:

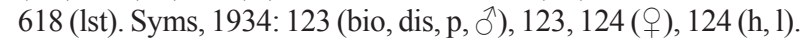

Wilson, 1938: 383 (rf). Eglin, 1939: 167 (rf), 172 (1). Fraser, 1940a: 180 (bio, la, p). Fraser, 1940b: 166 (rf). Mac Gillavry, 1949: 116-122 (dis). Berland \& Grassé, 1951: 18-22 (mf). Fraser, 1951: 226 (bio, fe). Grassé, 1951 (a). Condé \& Pagès, 1956a: 125 (rf). Condé \& Pagès, 1956b: 2 (dis), 1 (rf). Fraser, 1959: 2, 4 (mf), 13 (c), 35 (l). Metzger, 1960a: 199 (a). Wakemann, 1960: 128 (fe, 1). Vannier \& Condé, 1962: 97 (rf). Hoffmann, 1962: 261 (c), 265 (bio, mf), 265, 266 (a), 267 (dis). Kloet \& Hincks, 1964: 100 (lst). Metzger, 1966: 39 (a, dis). H. Aspöck \& U. Aspöck, 1969b: 34, 42 (dis). Kimmins, 1970: 356 (lst, tx). H. Aspöck \& U. Aspöck, 1973a: 52 (tx). Morgan, 1976: 231 (dis). Verdcourt, 1977: 1 (rf). Jong, 1978: 50 (dis, mf). Verdcourt, 1979: 59 (dis). Dorokhova, 1987: 34, 35 (dis, mf, ô). Barnard, 1988: 4 (a, c), 8 (dis, gen), 7 (ठ̋). Collins, 1989: 4 (bio). Stelzl, 1989: 13 (bio, fe). Czechowska \& Dobosz, 1991: 139 (lst). Kovarik et al., 1991: 356 (bio, 1). Marín \& Monserrat, 1991: 190 (bio), 190, 196 (fe), 192 (dis). Monserrat \& Triviño, 2013: 15 (1st).

Raphidilla maculicollis (Stephens, 1836): Navás, 1919: 63, 64 (a, mf), 51 (c), 64 (dis). Navás, 1923: 71 (c), 72 (dis). Navás, 1925: 47 (a, c, dis, 1st). Navás, 1928a: 21, 22 (c), 31, 32 (mf), 32 (a, đ). Navás, 1933a: 121 (1st). Navás, 1933b: 23 (dis). Navás, 1934a: 6 (dis). Navás, 1934b: 31 (c), 41 (a, mf), 42 (dis). Steinmann, 1964: 216 (a, c). Steinmann, 1967 (rf). Monserrat \& Triviño, 2013: 15 (lst).

Raphidia (Raphidilla) maculicollis Stephens, 1836: Condé \& Pagès, 1956a: 130 (dis). Condé \& Pagès, 1959: 192, 193 (lst). Berland, 1962: 21 (mf, rf). Monserrat \& Triviño, 2013: 15 (lst). Agulla maculicollis (Stephens, 1836): Carpenter, 1936: 104 (bio), 107 (p). Kloet \& Hincks, 1945: 80 (1st). Mac Gillavry, 1949: 121 (rf). Blair, 1951: 160 (dis, rf). H. Aspöck \& U. Aspöck, 1964: 141 (dis), 218 (c), 258 (a), 259 (đ) , 293, 295 (bio). Bartoš, 1967: 330 (a, rf). H. Aspöck \& U. Aspöck, 1969a: 38 (rf). Geijskes, 1972: 45 (rf). Kowalska, 1978: 213 (lst). Monserrat \& Triviño, 2013: 15 (lst).

Raphidia (Atlantoraphidia) maculicollis Stephens, 1836: H. Aspöck \& U. Aspöck, 1968b: 60 (tx), 60, 61 (a, §), 61 (mf). H. Aspöck et al., 1974a: 46 (rf), 48 (1), 49 (dis). H. Aspöck et al., 1974c: 2, 15 (lst), 4 (1), 14, 15 (dis), 16 (rf), 26 (pa). H. Aspöck, 1977: 17, 18 (dis). Eglin-Dederding, 1979: 494 (dis). H. Aspöck et al., 1980a: vol. I: 38, 39 (c), 117 (bio, 1, tx), 118 (rf), vol. II: 36 (ðึ), 41 (ㅇ), 165 (a), 252 (dis). DíazAranda et al., 1986: 1134 (dis). Gepp 1986a: 138 (1). Monserrat 1986a: 96 (dis). Monserrat \& Díaz-Aranda 1987: 175 (dis). Díaz-Aranda \& Monserrat 1988a: 218 (dis), 229 (bio). Séméria \& Berland 1988: 35 (rf). Marín \& Monserrat 1990: 222 (bio, dis, fe). Dobosz 1991a: 201 (1), 201, 202 (dis). Monserrat \& Triviño, 2013: 15 (1st).

Atlantoraphidia maculicollis (Stephens, 1836): H. Aspöck 1986: 16, 17 (a). H. Aspöck 1990: 56 (dis). H. Aspöck et al., 1991: vol. I: 69, 347, 348 (ङ) , 73, 347, 348 (q), 89, 348, 350 (bio), 125, 128, 130 (c), 129, 131, 132 348, 350, 547, 550, 551, 576, 577 (dis), 347, 349 (a), 348 (tx), 348, 349 (1), 348, 350 (p), 614 (lst), vol. II: 52 (mf), 58, 74 (a), 173 (ふ), 270 (ㅇ), 354 (1), 486 (dis). Plant, 1992: 119 (bio), 119, 127 (dis). Retzlaff \& Schulze, 1992 (dis). Hollier \& Belshaw, 1993: 170, 171 (bio, dis, gen). Plant, 1994: 17 (lst), 29 (fe), 32, 33 (dis), 33 (bio). Marín \& Monserrat, 1995: 112 (bio, dis). H. Aspöck \& Hölzel, 1996: 44 (dis). Monserrat, 1996: 12 (bio, 1st). Saure, 1996: 80 (dis). H. Aspöck, 1997: 113, 114 (bio). Plant, 1997 (dis, tx). H. Aspöck, 1998a: 19 (a, dis). H. Aspöck, 1998b: 37 (dis). Röhricht \& Tröger, 1998: 233 (dis). Cross, 2000: 4 (bio). H. Aspöck et al., 2001: 50, 331, 337 (dis, tx). U. Aspöck et al., 2001: 76 (mf), 75 (lst). Plant, 2001: 183 (bio, dis). Monserrat \& Papenberg, 2006: 204 (rf). Monserrat \& Papenberg, 2010: 63 (rf), 63, 64 (dis). Haring et al., 2011: 4 (dis, 1st), 10, 12 (tx), 
fig.S2 (f). Ocharan et al., 2012: 423 (bio, dis). Monserrat \& Papenberg, 2012: 292 (rf). H. Aspöck \& U. Aspöck, 2013: 34 (lst), 62 ( 9 ), 85, 86, 102 (eco), 133 (gen). Monserrat \& Triviño, 2013: 15, 38 (dis, 1st, tx).

= Raphidia centrodes Navás, 1915: Navás, 1915b: 865 (d), 865867 (mf), 866 (ô, ㅇ), 866, 867 (a). H. Aspöck \& U. Aspöck, 1969a: 38 (sin).

$=$ Raphidilla centrodes (Navás, 1915): Navás, 1919: 51 (c), 61 (§) , 61, 62 (a, mf), 62 (dis). Lestage, 1928: 32 (a). Navás, 1933a: 121 (1st). Navás, 1934a: 6 (dis). Steinmann, 1964: 216 (a), 217 (c). Steinmann, 1967 (rf).

= Raphidia affinis Stephens, 1836: Curtis, 1824: 37, 38 (rf). Stephens, 1829 (lst). Stephens, 1836: 131 (d). Schneider, 1843 : 73 (l, mf, mon). Brauer, 1855a: 723 (dis). McLachlan, 1868: 162 (rf), 220 (sin). McLachlan, 1870: 18 (rf). Albarda, 1889 (sin). Albarda, 1891: 73, 76, 78, 81 (sin). Ramme, 1911: 12 (dis). Kimmins, 1970: 352 (tx).

Raphidia hispanica Rambur, 1842: Pictet, 1865: 52 (a), 52, 53, 119 (mf), 53 (dis) - ie.

Raphidia ophiopsis Linnaeus, 1758: Waterhouse, 1836: 24-27 (bio, 1, p) - ie. Hagen, 1858: 30 (mf) - ie.

TIPO: lectotipo $q$ depositado en el Natural History Museum (Londres).

\section{LOCALIDAD TÍPICA: Inglaterra.}

MATERIAL ESTUDIADO: ESPAÑA: Álava: Arluzea-San Justi, Bernedo, Izki Parque Natural, 30TWN3830, 850-1.000 m, 4.VI.2004, 1 9, S. Pagola Carte (VM), Okelu (Korres), ArraiaMaeztu (Izki Parque Natural), 30TWN4628, 770 m, 18.V.2003, 1 +, S. Pagola Carte (VM). Ávila: Hoyos del Espino, 30TUK1568, $1.440 \mathrm{~m}, 10$. VI.06, 1 ते sobre Pinus sylvestris, M. Portillo (USAL). Cuenca: Ceja de Cañada, Poyatos, 30TWK8175, 1.250 m, 26.V.1985, 1 กै, sobre P. nigra, leg.? (VM). Guadalajara: Cantalojas, 30TVL698647, $1.620 \mathrm{~m}, 10$. VII.1988, 1 우 sobre hierba, F. Marín, 11.VI.1989 1 ते sobre Fagus sylvatica 25.VI.1989, 1 ô/ 1 + sobre F. sylvatica, 1 q sobre Quercus pyrenaica, F. Marín (VM), Galve de Sorbe, 30TVL6483, 1.394 m, 17.VI.1989, 1 s sobre P.sylvestris, V. J. Monserrat (VM), Molina de Aragón, 30TWL9822, 1.200 m, VII.1973, 1 9, M. A. Herranz (VM), Peñalen, 30TWL7802, 1.369 m, 15.VI.1984, 2 §ิ ô sobre P. sylvestris, V. J. Monserrat (VM), Tamajón, 30TVL7939, 1.029 m, 9.VII.1989, 1 ㅇ, sobre Cistus ladanifer, F. Marín (VM),

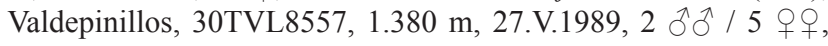
sobre P. sylvestris, F. Marín (VM), Villacadima, 30TVL8170, 1.341 m, 28.V.1988, 1 ㅇ, 28.IV.1989, 1 ô, sobre P. sylvestris, F. Marín (VM). Huesca: Hecho, 30TXN8434, 860 m, 11.VI.1983, 1 q sobre hierba, V. J. Monserrat (VM), Parzán, 31TBH7126, 1.150 m, 13.VI.1983, 1 † sobre P. sylvestris, V. J. Monserrat (VM), Puerto de Monrepós, 30TYM1592, 1.260 m, 31.V.1986, 1 ㅇ, leg.? (MNCN). León: San Emiliano, 30TTN5562, 1.189 m, 23.V.1992, 1 †, J. E. Marcos (UNIL), Tabullo del Monte, 29TQG3088, 985 m, 12.VII.1984, 1 q, E. Quirós (UNIL). Logroño: Puerto de Piqueras, 30TWM3855, $1.710 \mathrm{~m}, 1 . \mathrm{V} .1980,1$ त en hojarasca $F$. sylvatica, L. S. Subías (VM). Madrid: Cercedilla, 30TVL1010, 1.188 m, VII.1914, 1 స̂, Avían (MNCN), El Escorial, Monte Abantos, 30TVK0296, $1.750 \mathrm{~m}, 26 . \mathrm{VI} .1977,2$ ऊิते sobre $P$. sylvestris, R. Outerelo (VM), El Ventorrillo, 30TVL1312, $1.480 \mathrm{~m}$, 20.V.1988, 1 ㅇ, leg.? (MNCN), 1.VI.1988, $1 \hat{\jmath} / 2$ 우 J. L. Nieves \& Rey (MNCN), 14.VII.1988, 1 ㅇ, leg.? (MNCN), La Hiruela, 30TVL6147, 1.300 m, 8.III.1984, 1 §ิ (ex larva)+1 larva sobre $Q$. pyrenaica, A. Baz (VM), 10.V.1984, 3 ถิ ठิ sobre Erica arborea, A. Baz (VM), 12.VI.1984, 2 ๙̋ / 1 ㅇ, A. Baz (VM), 5.VIII.1984, 1 ふै, A. Baz (VM), 13.VI.1986, 1 ค, V. J. Monserrat (VM), 16.V.1987, 1 ㅇ capturada como presa de cebo de Ficedula hypoleuca, Pott (VM), 28.V.1988, 1 ô, sobre Q. pyrenaica, 29.V.1989,
11.VI.1988, 1 ㅇ, en ribera, 29.V.1989, 1 ऽ sobre $Q$. pyrenaica, F. Marín (VM), La Pedriza, 30TVL21, 1.200 m, 22.V.1992, 2 우, M. Costas (VM), Montejo de la Sierra, 30TVL5545, $1.152 \mathrm{~m}$, VI.1936, 1 \&, D. Peláez (MNCN), Montejo de la Sierra, Hayedo de Montejo, 29.V.1977, 1 §, R Outerelo (VM), 4.VI.1988, 1 ㅇ, 11.VI.1988, 1 + sobre F.sylvatica, F. Marín (VM), 14.VI.1989, 2

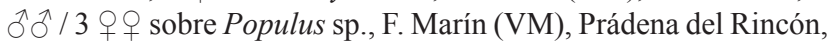
30TVL5444, 1.104 m, 26.VI.1989, 1 ㅇ, F. Marín (VM), Puerto de Canencia, 30TVL3524, $1.500 \mathrm{~m}, 24 . \mathrm{V} .1976,1$ ㅇ sobre $P$. sylvestris, Ladera Picolapala, 24.V.1976, 1 ภ/ 2 우 sobre P. sylvestris, V. J. Monserrat (VM), Puerto de Cotos, 30TVL1919, 1.840 m, 27.VI.1975, 2 ठぇ / 5 우 sobre P. sylvestris, V. J. Monserrat

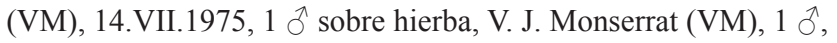
sobre P. sylvestris, V. J. Monserrat (VM), 23.V.1976, 1 ô sobre tocón de pino, R. Outerelo (VM), Río Lozoya, 4.VI.1975, 1 ô sobre P. sylvestris, V. J. Monserrat (VM), Puerto de la Morcuera, 30TVL2920, 1.600, 4.VI.1975, 2 के ô sobre P. sylvestris, V. J. Monserrat (VM), Puerto de Navafría, 30TVL3137, $1.700 \mathrm{~m}$, 4.VI.1979, 1 đ̂ sobre P. sylvestris, C. P. Iñigo (VM), Rascafría, El Paular, Los Hoyones, 30TVL2527, 1.163 m, 31.V.1976, 1 ㅇ, V. J. Monserrat (VM), Tablada, 30TVL0506, 981 m, IV.1925, 1 ㅇ, leg.? (MNCN), Valle de Fuenfría, 23.V.1954, 2 $\widehat{\partial} / 1$ o, W. Steiner (VM), 10.VI.1976, 1 त sobre P. sylvestris, R. Outerelo (VM). Navarra: Arangoiti, Irunberri, 30TXN42, 1.200 m, 23.V.2003, 1 o / 1 q, S. Pagola Carte (VM), Erromantzatua, 30TXN42, 670 m, 23.V.2003, 1 ㅇ, I. Zabalegui (VM), Igúzquiza, 30TWLN72, 515m, 28.VI.1983, 1 †, L. S. Subías, Ruiz y Fernández (VM). Orense: Alto del Couso, 29TPG0985, 700 m, 4.VI.1977, 1 q sobre Q. robur, V. J. Monserrat (VM), Arcos, 29TNG7699, 400 m, 29.VI.1977, 1 ㅇ sobre Q. suber, V. J. Monserrat (VM), Chandreja de Queixa, 29TPG3379, 940 m, 30.VI.1977, 1 ㅇ, V. J. Monserrat (VM), Freanes, 29TNG76, $100 \mathrm{~m}, 4 . \mathrm{VI} .1977,1$ q sobre $Q$. robur, V. J. Monserrat (VM), Herdadiña, 29TNG7542, $400 \mathrm{~m}$, 28.VI.1977, 1 q sobre Arbutus unedo, V. J. Monserrat (VM), Herosa, 29TPG5052, $880 \mathrm{~m}, 6 . \mathrm{VI} .1977,1$ \& sobre $Q$. robur, V. J. Monserrat (VM), Laguna de Antela, 29TPG0561, $620 \mathrm{~m}$, 28.VI.1977, 1 q sobre P. pinaster, V. J. Monserrat (VM), Luintra, 29TPG0495, 680 m, 5.VI.1977, 1 q sobre Cytisus sp., V. J. Monserrat (VM), Manzaneda, 29TPG4585, 1.471 m, 23.V.1981, 1 , F. Novoa (VM), Rubia, 29TPH6901, 1.857 m, 6.VI.1981, 1 연, F. Novoa (VM), Yermes, 29TNG8852, 940 m, 28.VI.1977, 2 우우 sobre P. sylvestris, V. J. Monserrat (VM). Salamanca: Batuecas (Monasterio), 29TQE4383, 600 m, 4.VI.1980, 1 ․ E. Galante (VM), El Maillo, 29TQE3894, 900 m, 1.V.1982, 1 ô/ 1 o, C. Urones (VM), La Hoya, Béjar, 30TTK716, 1.260 m, 26.VII.2008, 1 \&, sobre Q. pyrenaica, V. J. Monserrat (VM), Puerto de Perales, 29TPE9657, $900 \mathrm{~m}, 15 . \mathrm{V} .1980,1$ 을 sobre P. pinaster, V. J. Monserrat (VM). Segovia: Chañé, 30TUL8077, 767 m, de 18-V a 3.VII.2000, 1 q, J.F. Gómez. (VM), El Espinar, 30TUL9408, 1.191 m, 3.VII.1984, 1 , C. Ornosa (VM), La Granja de San Ildefonso, 30TVL1528, 1.193 m, VI.1908, 1 §े, Arias (MNCN), 1 ㅇ J. Sanz (MNCN), Riofrío de Riaza, 30TVL6266, 1.312 m, 28.V.1988, 1 q sobre brezo, 9.VII.1988, 1 ㅇ sobre $F$. sylvatica, 14.VI.1989, 1 ㅇ al vuelo, F. Marín (VM), San Rafael, 30TUL9907, 1.180 m, 1 ふै, C. Bolívar (MNCN), 1 q G. Menor (MNCN), Valsaín 30TVL1326, $1.180 \mathrm{~m}, 1$ +, J. Bajo (VM), VII.1933, 1 , R. Ajenjo (MNCN), 25.V.1995, 1 ㅇ, Munguira (UAM), 27.V.1997, 1 ㅇ, leg.? (UAM). Soria: Montenegro de Cameros, 30TWM2059, 1.240 m, 1 ๙ / 1 ㅇ, C. Bolívar (MNCN), Puerto del Mojón Pardo, 30TWM0230, 1.234 m, 20.V.1989, 1 त sobre P. sylvestris, F. Marín (VM). Teruel: Puerto de Cuarto Pelado, 30TYK1390, 1.610 m, 19.VI.1987, 2 ઈै / 1 क sobre P. sylvestris, V. J. Monserrat (VM). Valladolid: Valladolid, 30TUM51, 691 m, 14.VI.1993, 1 †, P. Zumel (UNIL). Zamora: Boya, Sierra de la Culebra, 29TQG1944, 878 m, 30.V.2004, 1 q, 
F. Novoa (VM), Lubián, 29TPG7355, 1.000 m, 14.VI.1976, 1 ㅇ, V. J. Monserrat (VM), Requejo, 29TPG8655, 962 m, 7.V.1977, 2

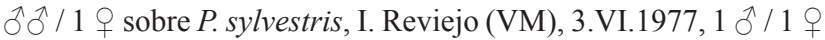
sobre P. sylvestris, V. J. Monserrat (VM), 23.VI.1977, 4 q 9 , V. J. Monserrat (VM), 2 우 sobre P. sylvestris, V. J. Monserrat (VM). Zaragoza: Alto del Moncayo, 30TWM9728, 1.350 m, 3.VI.1994, 1 Oे, sobre P. sylvestris, 1 + , sobre hierba, 1 , , sobre Genista sp., 9.VII.1994, 1 q, sobre P. sylvestris F. Marín (VM).

Atlantoraphidia maculicollis es una especie monotípica que presenta claras diferencias morfológicas tanto en el $\hat{\partial}$ como en la $q$.

MORFOLOGÍA EXTERNA: El clípeo es pardo claro y el labro pardo oscuro. En las antenas el escapo es pardo oscuro, siendo los artejos más basales del flagelo ocres y el resto pardo. En los apéndices locomotores las coxas son pardas, los trocánteres y los fémures II y III pardos, y el resto de los segmentos son amarillos ocre. En el abdomen los terguitos son negros, con dos manchas caudales amarillas. Los esternitos son pardos con un borde caudal amarillo.

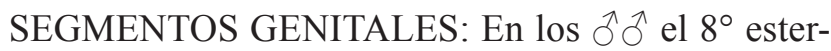
nito se encuentra íntimamente ligado al $9^{\circ}$ segmento (fig. 57). El $9^{\circ}$ terguito se prolonga en dirección caudal. El $9^{\circ}$ esternito es largo (figs. 57, 58). Los gonocoxitos se alargan dorsoventralmente estando muy marcado el apodema del estilo (fig. 57). La región dorsal del gonocoxito es pequeña y la ventral es grande, con su base ligeramente abombada y con un pequeño proceso en forma de espina (fig. 57). Los estilos son grandes y se bifurcan ampliamente con un ápice en dirección dorsal y otro en dirección caudal (fig. 57). El ectoprocto se ensancha algo en su extremo anterior (fig. 57). La hipovalva es par, con bandas esclerotizadas y su base está débilmente ensanchada y apenas es divergente (fig. 58). Los parámeros son pares, teniendo su parte dorsal forma de cuerno y la ventral forma de placa (fig. 57). Según H. Aspöck et al. (1991) la parte basal podría ser una estructura derivada de la hipovalva o del endofalo. No se localiza un gonarco. El hipandrio interno es muy delicado (fig. 84).

En las $q Q$ el $7^{\circ}$ esternito está prolongado con el borde caudal convexo (fig. 107). El intersegmento S $7 / 8$ es largo y membranoso (fig. 107). El $8^{\circ}$ terguito presenta en el borde cefálico un saliente en dirección anterior reduciéndose en posición anteroventral de forma oblicua (fig. 107). Respecto a la genitalia interna, el atrio de la bolsa copuladora se encuentra fuertemente esclerotizado en forma de cápsula (figs. $129,130)$, observándose en vista dorsal un dibujo en forma de herradura característico (fig. 130). El ductus saculi es corto (fig. 129). El sáculo es poco llamativo y de paredes delicadas (fig. 129). El ductus receptaculi sale de la parte media del sáculo. El receptáculo seminal es piriforme, siendo las glándulas cortas y claviformes (fig. 129).
BIOLOGÍA: Los imagos tienen tendencia a evitar biotopos templados, y poseen una distribución altitudinal global entre los 10-1.840 m. La densidad de individuos en las poblaciones suelen ser altas. En la Península Ibérica la mayoría de los individuos han sido capturados en medios de tendencia húmeda, sobre macrofanerófitos de montaña en bosques aciculifolios (Pinus), en particular sobre Pinus sylvestris. También existen registros sobre haya (Fagus sylvatica), madroño (Arbutus unedo), melojo (Quercus pyrenaica), roble (Q. robur), chopos (Populus) o sobre vegetación arbustiva (Erica, Cytisus). Se conoce la existencia de un parásito en el Reino Unido: Nemeritis $s p$.

FENOLOGÍA: La actividad de los imagos es monovoltina, estacionaria primaveral y estival, transcurriendo durante los meses de IV-VI con citas puntuales en VII. En la Península Ibérica las citas transcurren durante los meses III-VIII.

VARIABILIDAD: La variabilidad es escasa, afectando a individuos de forma aislada de coloración relativamente oscura.

ESTADOS PREIMAGINALES: En los segmentos abdominales de la larva, la figura dorsal llega hasta el borde caudal (fig. 148). La banda media dorsal recorre toda la zona media perdiendo intensidad en la parte anterior (fig. 148). Las bandas laterales son irregulares, ensanchándose en la parte caudal. La figura lateral sobrepasa claramente la sutura dorsal (fig. 148). La figura ventral se encuentra poco desarrollada. Las larvas son edáficas y corticícolas. Hay registros de larvas en la hojarasca de pinares, de forma aislada bajo cortezas de Pinus, sobre todo en la base del árbol. Mediante cría ex ovo se ha determinado un periodo de desarrollo de 2 años. La última hibernación es en fase de pupa, y por tanto es un ciclo vital de tipo II (fig. 18). El estadio de pupa se mantiene entre 7-9 meses. Morfología y aspecto de la larva en figura 148 y en H. Aspöck et al. (1991).

DISTRIBUCIÓN: Europa occidental y central. Centro y norte de la Península Ibérica (fig. 161). Elemento faunístico expansivo, monocéntrico de carácter atlantomediterráneo. Existen citas de Rambur (1842) que presentan muchas dudas, y por tanto no han sido incluidas en los mapas de distribución.

DISCUSIÓN: Atlantoraphidia maculicollis es la única especie europea, al norte de los Pirineos, que presenta una vena apical. Esta especie ha sido citada junto a $S$. aliena, $H$. castellana, $H$. laufferi, O. baetica bolivari, y Fibla (Fibla) hesperica. Respecto a las especies citadas en la Península Ibérica es morfológicamente muy parecida a $H$. castellana, pudiéndose distinguir con seguridad sólo mediante el estudio de la genitalia en ambos sexos. Los $\partial^{\lambda} \widehat{\jmath}$ de Atlantoraphidia maculicollis se diferencian en la forma del estilo, la 
hipovalva y el proceso del gonocoxito (figs. 57, 58, 63-65), y en las $q$ 우 en la forma del atrio de la bolsa copuladora y la falta de salientes en posición subterminal en el receptáculo seminal (figs. 129, 130, 133, 134). También suele ser parecida y por tanto posible causa de confusión con $H$. laufferi, aunque esa última suele ser más pequeña de tamaño y la genitalia, aunque parecida en el $\hat{\sigma}$, se diferencia con facilidad al carecer de un proceso del gonocoxito en forma de espina (figs. 57, 58, 61, 62).

\section{Género Harraphidia Steinmann, 1963}

Harraphidia Steinmann, 1963: 188 (d, a), 186 (c), [especie tipo por designación original: Harraphidia harpyia Steinmann, 1963].

Considerado como subgénero de Raphidia Linnaeus, 1758: H. Aspöck \& U. Aspöck, 1968b: 63 (tx). U. Aspöck \& H. Aspöck, 1969: 168 (tx). H. Aspöck et al., 1975: 371 (1). H. Aspöck et al., 1980a: 87 (dis). H. Aspöck et al., 1980b: 78 (p).

Considerado como género: Harraphidia Steinmann, 1963: 188 (d, a), 186 (c). Buresch \& Popov, 1973: 155 (rf). H. Aspöck, 1986: 25-26 (dis). H. Aspöck et al., 1989: 89 (dis). H. Aspöck, 1990: 50 (1st), 53 (tx), 54-55 (dis). H. Aspöck et al., 1991: vol. I: 350 (a, mf, đ) , 54, 76, 351 (bio), 351, 552, 555, 559 (dis), 107, 111, 115, 351 (tx) 117, 351 (ㅇ) 351 (1), vol. II: 424 (dis). Oswald \& Penny, 1991: 64 (1st). U. Aspöck \& H. Aspöck, 1994: 39 (tx). H. Aspöck \& Hölzel, 1996: 38 (gen), 44 (dis). H. Aspöck, 1998b: 39 (tx). H. Aspöck et al., 2001: 19, 50 (dis, tx). H. Aspöck, 2002: 38 (1). H. Aspöck \& U. Aspöck, 2007: 99 (dis). Monserrat \& Papenberg, 2006: 204, 206 (tx), 206 (a, ô, mf), 206, 207 (), 207, 208, 210 (bio), 210 (dis), 211, 212 (c). Monserrat \& Papenberg, 2010: 63 (gen). Haring et al., 2011: 3 (dis), 12 (tx), fig.S3 (dis). U. Aspöck et al., 2012: 577 (lst), 577, 579, 580 (f), 580 (biogeo). Monserrat \& Papenberg, 2012: 292 (rf). H. Aspöck \& U. Aspöck, 2013: 12 (tx), 40 (gen).

= Flavoraphidia H. Aspöck \& U. Aspöck, 1968b: 61 (d, a, ô, , $\mathrm{mf}, \mathrm{tx}$ ), [especie tipo por designación original: Raphidia laufferi Navás, 1915]. Monserrat \& Papenberg, 2006: 206 (tx).

Considerado como subgénero de Raphidia Linnaeus, 1758: U. Aspöck \& H. Aspöck, 1969: 168 (dis). H. Aspöck et al., 1975: 371 (1). H. Aspöck et al., 1980a: vol. I: 87 (dis, gen), 86 (tx, sin), vol. II: 252 (dis). Oswald \& Penny, 1991: 63 (1st). Monserrat \& Papenberg, 2006: 206 (tx).

Considerado como subgénero de Harraphidia Steinmann, 1963: Monserrat \& Papenberg, 2006: 211 (d, a, ô, 우).

El género Harraphidia está formado por cuatro especies pertenecientes a los subgéneros Harraphidia y Flavoraphidia, citados del centro y sur de la Península Ibérica (figs. 162, 163) y noroeste de África. En la península es conocida por las especies Harraphidia (Flavoraphidia) laufferi y Harraphidia (Harraphidia) subdesertica.

\section{Harraphidia (Harraphidia) subdesertica (Monserrat \& Papenberg, 2006) \\ Harraphidia (Harraphidia) subdesertica Monserrat \& Papenberg, 2006: Monserrat \& Papenberg, 2006: 207, 213, 215 (d, a), 209, 210, 213, 215 (ð), 212 (c), 213 (mf), 213, 214 (ㅇ) , 213, 218 (rf), 215 (bio, fe), 215, 219 (dis), 220 (tx). Fernández, 2007: 388 (dis, lst). H. Aspöck \& U. Aspöck, 2013: 33 (lst), 141 (gen). Monserrat \& Triviño, 2013: 15, 39 (lst, dis).}

TIPO: holotipo $\widehat{\partial}$ depositado en Museo Nacional de Ciencias Naturales de Madrid.

\section{LOCALIDAD TÍPICA: Níjar, Almería (España).}

MATERIAL ESTUDIADO: ESPAÑA: Almería: Níjar, Carretera de Níjar, 30SWF7085, 350 m, 23.IV.1988, 1 ठ (a*, ठో*), M. A. Zarazaga (MNCN) (Tipo). España: Almería: Níjar, Carretera de Níjar, 30SWF7085, 350 m, 23.IV.1988, 3 ㅊํ / 1 우 (a, 우), M. A. Zarazaga (MNCN) (Paratipos). Cádiz: La Almoraima, Castellar, 30STF8117, 45 m, 8.IV.2003, 1 ㅇ, J. de Ferrer (MNCN) (Paratipo).

MORFOLOGÍA EXTERNA: Se trata de una especie de tamaño pequeño. La cabeza es de coloración negra. El clípeo es pardo oscuro y el labro ocre. En la antena el escapo, pedicelo y flagelo son pardos. El pronoto se encuentra bordeado por una banda parda. En los apéndices locomotores las coxas, trocánteres y fémures son pardos, siendo el resto de los segmentos ocres. La longitud del ala anterior mide entre 6.5-7 mm en el $\widehat{\jmath}$ (fig. 30) y entre 6-6.5 mm en la +. La venación alar es, casi en su totalidad, parda, siendo en el primer tercio basal del ala posterior de coloración ocre. Entre la C y el R hay una vena transversal al igual que entre la Sc y el R (fig. 30). En el abdomen la coloración de los terguitos y esternitos es parda.

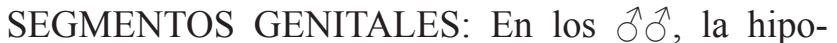
valva es par y presenta dos procesos anteriores que portan espinas y que convergen en los extremos hacia línea media (fig. 60), en su zona media la hipovalva posee un ensanchamiento globoso, ovoide, levemente recurvado hacia arriba en sus extremos laterales, y sus escleritos la recorren en toda su longitud divergiendo en su extremo caudal (figs. 59, 60), sus procesos caudales son marcadamente convergentes hacia su extremo (fig. 60).

En las 90 el intersegmento $7 / 8$ es corto y membranoso (fig. 108). El ductus receptaculi parte de la zona ventral del sáculo, es festoneado, no muy largo y no tiene forma de ovillo (fig. 132), su mitad basal posee su membrana interna muy plegada y rugosa, y porta diminutas espinas, y su mitad distal, en cambio, es muy lisa y de menor diámetro, presentando unos lóbulos verrugosos en su región terminal (fig. 132), en su tramo final su conducto aumenta de luz formando el receptáculo seminal que posee un par de glándulas largas y dilatadas en sus extremos (fig. 132).

BIOLOGÍA: Su biología es desconocida. Se ha hallado en similares medios que Harraphidia laufferi, aunque con muy escasa y baja amplitud altitudinal $(45-350 \mathrm{~m})$. Por las características ambientales de las zonas donde ha sido hallada, parece poseer una marcada preferencia por los medios xerotérmicos.

FENOLOGÍA: Su fenología es desconocida. La captura de los ejemplares hasta ahora conocidos se circunscriben a IV. 
ESTADOS PREIMAGINALES: Los estados preimaginales, tanto la larva como la pupa son desconocidos.

DISTRIBUCIÓN: España. Elemento faunístico ibérico estacionario, monocéntrico y xero-mediterráneo. Es de suponer que la distribución se limita exclusivamente a la zona árida del sur y sudeste de la Península Ibérica y, por su carácter xerotermo, quede circunscrita a estas zonas ibéricas de clima subdesértico y subtropical (fig. 162).

VARIABILIDAD: En el ejemplar de La Almoraima, Castellar (Cádiz) aparece en ambas alas una vena transversal entre la costa y el radio, y dos entre la subcosta y el radio.

DISCUSIÓN: Especie alopátrida y muy próxima a la especie Harraphidia (H.) divergens, conocida de Marruecos, al poseer semejante venación alar al existir en ambas especies las venas transversales entre $\mathrm{C} \mathrm{y}$ $\mathrm{R}$ como entre Sc y R. A su vez, existe en ambas especies un gran parecido en la genitalia del $\hat{\jmath}$, sobre todo en lo que respecta a la presencia de procesos membranosos en la hipovalva, pero difieren en su expansión y desarrollo y en la orientación de sus procesos y en la forma caudal de la propia hipovalva.

Harraphidia (Flavoraphidia) laufferi (Navás, 1915) Raphidia laufferi Navás, 1915: Navás, 1915b: 863 (d), 863, 868, 869 (a), 867-869 (mf), 868 (ふ)), 869, 871-874 (dis). Martín Albaladejo \& Izquierdo Moya, 2006: 134 (1st). Monserrat \& Triviño, 2013: 15 (1st).

Raphidia lanfferi Navás, 1915 (lapsus): Monserrat \& Triviño, 2013: 15 (lst).

Raphidilla laufferi (Navás, 1915): Bohigas \& Sanchez, 1917: 319 (dis). Navás, 1919: 50 (c), 59 (a, đ̋), 59-60 (mf), 60 (dis). Navás, 1921: 49 (dis). Navás, 1923: 71 (c), 72 (dis). Navás, 1928a: 21 (c), 28-29 (a, mf), 28 (ठ). Lestage, 1928: 31 (c). Navás, 1933a: 121 (lst). Navás, 1934a: 6 (dis). Navás, 1934b: 31 (c), 38-39 (mf), 39 (a, dis, ô). Steinmann, 1964: 216 (a), 217 (c). Monserrat \& Triviño, 2013: 15 (lst).

Raphidia (Flavoraphidia) laufferi Navás, 1915: H. Aspöck \& U. Aspöck, 1968b: 61 (a, mf, ô, o, tx). H. Aspöck et al., 1980a: vol. I: 39 (c), 117 (bio, tx), 118, 351 (dis), vol. II: 36 (ふ), 165 (a), 252 (dis). Díaz-Aranda \& Monserrat, 1988b: 215 (dis). Díaz-Aranda \& Monserrat, 1988c: 114 (dis). Monserrat \& Triviño, 2013: 15 (1st).

Raphidia (Hispanoraphidia) laufferi Navás, 1915: Monserrat \& Triviño, 2013: 15 (1st).

Harraphidia laufferi (Navás, 1915): H. Aspöck, 1990: 53, 57 (dis). H. Aspöck et al., 1991: vol. I: 129, 353, 576, 577 (dis), 130 (c), 349, 353, 355 (mf), 350, 353 (a), 351, 353 (), 353 (1, đ), 614 (lst), vol. II: 29-31 (mf), 41, 270 (ㅇ), 50-51 ()), 74 (a), 174 (ふ), 343, 354 (1), 487 (dis). H. Aspöck \& Hölzel, 1996: 44 (dis). H. Aspöck et al., 2001: 51 (tx), 51, 332, 338 (dis). Martín Albaladejo \& Izquierdo Moya, 2006: 134 (dis). H. Aspöck \& U. Aspöck, 2009: 56 (fe, 1, p). Haring et al., 2011: 4 (dis, 1st), 10 (tx), fig.S2 (f).

Harraphidia (Flavoraphidia) laufferi (Navás, 1915): Monserrat \& Papenberg, 2006: 206, 207 (a), 206, 210, 211, 220 (tx), 207, 214, 218 (ㅇ), 209, 210, 218 (ठ), 210, 216, 218 (l), 211, 212 (c), 218 (bio, fe, mf), 218, 219 (dis). H. Aspöck \& U. Aspöck,
2013: 33 (lst), 61 (d, ふึ), 85, 86, 101 (eco), 132, 133 (gen). Monserrat \& Triviño, 2013: 15, 39 (dis, lst, tx).

= Raphidia fuentei Navás, 1915: Navás, 1915b: 869-871 (a, d, dis, mf, đ̂). Fuente y Morales, 1929: 168-169 (mf), 169 (a). H. Aspöck et al., 1980a: 117 (sin). Monserrat, 2013: 287 (lst). Monserrat \& Triviño, 2013: 15 (lst).

= Raphidilla fuentei (Navás, 1915): Navás, 1919: 50 (c), 60 (す̊), 60-61 (a, mf), 61 (dis). Navás, 1923: 71 (c), 72 (dis). Navás, 1928a: 21 (c), 29-31 (mf), 30 (ð), 30-31 (a), 31 (dis). Lestage, 1928: 31 (c). Navás, 1933a: 121 (lst). Navás, 1934a: 6 (dis). Navás, 1934b: 31 (c), 40 (a, mf, đ̂), 41 (dis). Steinmann, 1964: 216 (a), 217 (c). Monserrat \& Triviño, 2013: 15 (1st).

TIPO: lectotipo $\widehat{\jmath}$ depositado en el Museo de Barcelona. LOCALIDAD TÍPICA: El Escorial, Madrid (España).

MATERIAL ESTUDIADO: ESPAÑA: Almería: Bédar, La Serena, 30SWG9116, 480 m, 1.V.2007, 2 ô sobre Anthyllis cytisoides, V. J. Monserrat (VM), $1 \delta / 1$ sobre Prunus dulcis, V. J. Monserrat (VM), 1 q sobre Tamarindus sp., V. J. Monserrat (VM), 1 $\lesssim$ sobre Lavandula pedunculata V. J. Monserrat (VM), 2.V.2007, 2 우 sobre $A$. cytisoides, V. J. Monserrat (VM), 1 ô sobre Pimpinella anisum, V. J. Monserrat (VM), 1 ते sobre L. pedunculata, V. J. Monserrat (VM), 1 $\widehat{~ / ~} 1$ + sobre Tamarindus sp., V. J. Monserrat (VM), 3 우 sobre Pistacea sp., 3.V.2007, 1 q sobre A. cytisoides, V. J. Monserrat (VM), 15.IV.2008, 1 ते sobre Pistacia sp., V. J. Monserrat (VM), El Ejido, Punta del Sabinar, 30SWF2660, 88 m, 12.IV.1992, 2 $\widehat{\partial} / 2$ 우 $ᄋ$ sobre Pistacia sp., V. J. Monserrat (VM), El Toyo, Rambla del Agua, 30SWF67, 140 m, 6.V.2011, $1+$ sobre Tamarix sp., V. J. Monserrat (VM), Gergal, 30SWG4008, 758 m, 8.V.2011, 2 우 sobre Tamarix sp., V. J. Monserrat (VM), Lubrín, Sierra de Bédar, Las Moletas, 30SWG877174, 590 m, 7.V.2013, 1 ô / 1 q sobre Artemisia sp., S. Pagola Carte (VM), Níjar, 30SWF7085, 350 m, 23.IV.1988, 1 q, M. A. Zarazaga (MNCN), Níjar, Barranco de Huebro, 30SWF7, 15.IV.2011, 1 đે, V. J. Monserrat(VM), Puerto de Alamillo, Rambla Honda, 30SWF7398, 1.V.2007, 2 ô sobre A. cytisoides, 7.V.2011, 1 \&, 1 ô/2 2 + sobre Olea europea, V. J. Monserrat (VM), Sorbas, La Herrería (Rio

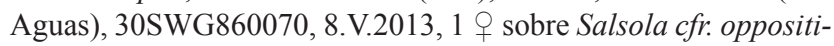
folia, S. Pagola Carte (VM), Tabernas, Sierra de Alhamilla, 30SWG5400, 400 m, 25.III.1960, 1 § / 3 우, Suárez (MNCN), 7.V.2011, 1 స/ 1 †, V. J. Monserrat (VM), Turre, Rambla Río Aguas 30SWG9812, 30.IV.2007, 8 ठ / 1 ㅇ sobre Tamarindus sp., V. J. Monserrat (VM), 1 sobre O. europea V. J. Monserrat (VM),

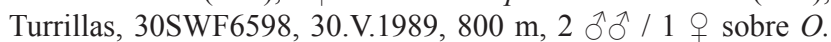
europaea, A. Baz (VM), 13.VI.2012, $1 \hat{\sigma} / 1$ o sobre Quercus rotundifolia, F. Acevedo (VM). Ávila: Barraco, Embalse de Burguillo, 30TTK67, 12.V.2007, 1 q sobre Juniperus oxycedrus, V. J. Monserrat (VM), La Cañada, 30TUK7395, $1.360 \mathrm{~m}$, 9.VI.1991, 1 ㅇ, A. Arillo (VM). Cádiz: Alcalá de los Gazules, 30STF5538, 120 m, 2.VI.1990, 2 ठิ ô sobre Genista tridentata, L. Díaz-Aranda (VM), Balneario del Pozo Amargo, 30STF8499, 160 m, 24.V.1977, 1 + , V. J. Monserrat (VM), Caños de Meca, 30SQA6608, 20 m, 22.V.2004, 1 i sobre O. europaea, V. J. Monserrat (VM), Cerro Alcántara, 30STF3364, 152 m, 23.V.1977, 1 q sobre Pistacia sp., V. J. Monserrat (VM), Estación de la Almoraima, 30STF8117, 25.V.2012, 1 q sobre Vinca sp., V. J.

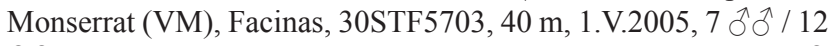
우 오 sobre O. europaea, V. J. Monserrat (VM), 25.V.2012, 1 우 sobre acebuche, V. J. Monserrat (VM), Jerez de la Frontera, 29SQA5664, 56 m, 11.V.1991, 1 ㅇ, A. Arillo (VM), 12.V.1991, 1 §., A. Arillo (VM), La Almoraima, Castellar, 30STF9790, 45 m, 8.IV.2003, 1 त, J. de Ferrer (MNCN), Olvera, 30STF9790, 643 m, 28.IV.1986, 1 § / 1 ㅇ, M. Baena(VM), Puerto Perales, 29TPE9657, 900 m, 15.V.1980, 2 우 sobre Pinus pinaster, V. J. Monserrat 
(VM), Punta Carnero, 30STE8195, 6 m, 12.V.2002, 2 ઈิ $/ 2$ 우 J. de Ferrer (MNCN), Tarifa, Los Lances, 30STE6390, $10 \mathrm{~m}$, 9.V.1991, 1 त/ / 1 ㅇ, J. de Ferrer (MNCN). Córdoba: Los Pedroches, 30SUH3285, $530 \mathrm{~m}, 1$.V.2000, 1 ते sobre O. europaea, leg.? (EEZ). Granada: Arenales, 30SVG43, 783 m, 1.V.1999, 4 ઈิ시 / 2 우 sobre O. europaea, leg.? (EEZ), Cerro Gordo, Cantavieja, 30SVF26, 30 m, 2.IV.2011, 1 q errante, V. J. Monserrat (VM), Colomera, 30SVG3636, 737 m, 1.V.1999, 2 우 sobre $O$. europaea, leg.? (EEZ), 1.VI.1999, 2 $\widehat{\jmath} \widehat{\jmath}$, leg.? (EEZ), Dehesa del Generalife, s.f., 1 $\widehat{\sigma}$, M. Morente (VM), Deifontes, 30SVG5130, 737 m, 1.V.1999, 13 ठૈ $/ 8$ 우 sobre O. europaea, leg.? (EEZ),

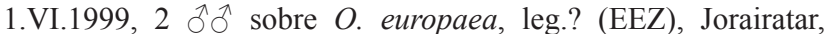
30SVF9086, 800 m, 1.V.1986, 1 ㅈ/ 2 웅 sobre Populus sp., V. J. Monserrat (VM), Loja, 30SVG9714, 550 m, 1.VI.1986, 1 ô, 1 ㅇ sobre Celtis australis, leg.? (VM), Los Tablones, 30SVF5969, 6.V.2011, 1 ते sobre P. halepensis, V. J. Monserrat (VM). Orgiva, 30SVF6284, 435 m, 17.VI.2010, 3 ふ઼ / 2 우우 sobre Pistacea, S. Pagola (VM), Pozo Alcón, Castril, 30SWG1684, $1.050 \mathrm{~m}$, 5.V.2013, 1 o sobre Q. ilex, S. Pagola Carte (VM), Puerto de la Mora, 30SVG5825, 1.390 m, 13.VII.2007, 1 q sobre Retama monosperma, V. J. Monserrat (VM), Puerto de Onitar, 30SVG4544, 900 m, 1.VI.1986, 1 § / 1 q sobre O. europaea, leg.? (VM), 1.VII.1986, 1 \& sobre O. europaea, V. J. Monserrat (VM), Vélez de Benaudalla, 30SVF5476, $170 \mathrm{~m}, 5 . \mathrm{V} .2011,1$ ऽे sobre hierba, V. J. Monserrat (VM), Venta del Chaleco, 30SVF8981, $1.200 \mathrm{~m}$, 2.VI.1986, 2 qㅇ, V. J. Monserrat (VM), Zaidín, Parque de Invierno, 30SVG4612, $1.000 \mathrm{~m}, 22 . \mathrm{VI} .1982,1 \hat{\jmath}$, M. Campos (VM), Zujar, 30SWG1355, $761 \mathrm{~m}, 19 . \mathrm{IV} .1981,1$ 이 / 1 ㅇ sobre $P$. halepensis, V. J. Monserrat (VM), Valdenoches, 30TVL9203, 800 m, 14.VI.1984, 1 o sobre P. halepensis, V. J. Monserrat (VM), 21.V.1988, 1 त sobre P. halepensis, F. Marín (VM). Huelva: Aracena, 29SQB1496, 732 m, 19.V.1977, 1 ․, V. J. Monserrat (VM), Coto de Doñana, 29SQA3090, 12 m, 6.VI.1983, 1 ㅇ, C. Montes (VM), La Corte, 29SPB9992, 10 m, 23.V.2012, 1 సै sobre Pistacea lentiscus, V. J. Monserrat (VM). Jaén: Campo Redondo, 30SVH9936, 500 m, 4.VI.1986, 1 q sobre P. lentiscus, V. J.

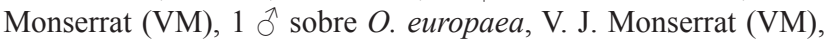
Collado de los Jardines, 30SVH5648, 850 m, 2.VI.1989, 1 o sobre Pistacia, A. Baz (VM), 20.VI.2004, 600 m, 1 స sobre J. oxycedrus, V. J. Monserrat (VM), Escañuela, 30SVG0992, 300 m, 30.V.1986, 1 ô sobre O. europea, V. J. Monserrat (VM), La Aliseda, 30SVH4943, 700 m, 31.V.1986, 1 ○/ 1 ㅇ sobre Q. ilex, leg.? (VM), Puerto de Despeñaperros, Salto del Fraile, 30SVH5548, 600 m, 31.V.1986, sobre Q. ilex, 1 ô, V. J. Monserrat (VM), 1 o sobre J. oxycedrus, V. J. Monserrat (VM), 1 앙 sobre Fraxinus, V. J. Monserrat (VM), Río Despeñaperros, Santa Elena, 30SVH54, 742 m, 20.VI.2004, 1 o sobre Cistus ladanifer, V. J. Monserrat (VM), Sierra de Cazorla, Embalse Tranco de Beas, 30SWH1825, 650 m, 2.VII.1978, encinar, pinar, 1 ․, R. Outerelo (VM), Sierra Magina, 30SVG57, 1.VI.2000, 5 ठㅎ/ 9 우우 sobre O. europaea, leg.? (EEZ), Viñas, 30SVH1622, 600 m, 30.V.1986, $1 \hat{\delta} / 2$ 우 sobre P. pinea, leg.? (VM). Madrid: Alcobendas, 30TVK4688, 670 m, 10.VI.1981, 1 , V. J. Monserrat (VM), Alpedrete, 30TVL1301, 900 m, 28.VI.1975, 1 ․, V. J. Monserrat (VM). Aravaca, 30TVK3479, $650 \mathrm{~m}, 13$. VI.1991, 1 , V. J. Monserrat (VM). Batres, 30TVK2151, 600 m, 15.V.1978, 2 ๙ठ sobre O. europaea, V. J. Monserrat (VM), Boadilla del Monte, 30TVK2573, 689 m, 10.VI.1996, 1 ふै, D. Papenberg (VM), 16.VI.2002, 1 ते, D. Papenberg (VM), 25.VI.2002, 1 ô, D. Papenberg (VM), 12.VI.2005, 1 ふ, D. Papenberg (VM), 20.VII.2008, 1 +, D. Papenberg (VM), Cantoblanco, Universidad Autónoma de Madrid, 30TVK4188, 678 m, 1998, 1 §̂, E. García Barros (UAM), Casa de Campo, 30TVK3675, 650 m, 25.V.1972, 1 त, R. Outerelo (VM), 17.V.1974, 1 / / 1 ㅇ, V. J. Monserrat (VM), 26.V.1985, 1 \&, M. Candela (UAM), Cercedilla,
30TVL1010, 1.188 m, 13.VI.1982, 1 ô, R. Outerelo (VM), Cercedilla, La Cabezuela, 20.VII.1973, 1 đ, V. de Paz (VM), Ciudad Universitaria, 30TVK3877, 700 m, 1.VI.1971, 1 §,, S. Arnaiz (VM), 8.V.1972, 1 ․, J. Hernández (VM), 9.V.1972, 2 ฮึ ฮౌ / 1 ㅇ, J. Calle (VM), 17.V.1972, 1 ઈ/ 1 ㅇ, V. J. Monserrat (VM), 1.VI.1976, 1 오, V. J. Monserrat (VM), 16.V.1978, 3 ๙ぇ / 8 오우 sobre hierba, V. J. Monserrat (VM), Chapinería, 30TUK9770, 680 m, 3.VI.1978, 1 q sobre R. spaerocarpa, V. J. Monserrat (VM), Dehesa de la Villa, 9.VI.2014, $2 \hat{\delta} \delta / 1$ 우 sobre P. pinea, V. J. Monserrat (VM), El Escorial, 30TVL9203, 909 m, 2 우, Lauffer

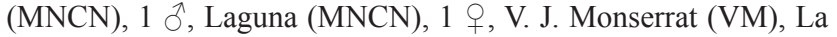
Herrería, 30TVK0292, $1.000 \mathrm{~m}$, 24.II.1976, 1 larva sobre $Q$. suber, R. Outerelo (VM), El Ventorrillo, 30TVL1312, $1.480 \mathrm{~m}$, 20.VII.1989, 1 \& , J. L. Nieves \& Rey (MNCN), 11.VII.1991, 1 + A. Garrido (MNCN), Fte. del Cervunal, Abantos, 7.VII.2007, 1 우 sobre P. sylvestris, 21.V.2008, 1 क sobre Cytisus purgans, V. J. Monserrat (VM), Fuente de la Teja, 30TVK0296, 21.VI.2008, 1 ठ sobre hierba, V. J. Monserrat (VM), El Pardo, 30TVK3481, 620 m, 14.V.1971, 1 đ̃, J. Martín (VM), Hoyo de Manzanares, 30TVK2397, 950 m, 1.VII.1975, 1 ㅇ, A. Soler (VM), 23.VI.1980, 1 q sobre J. communis, V. J. Monserrat (VM), La Jarosa, Guadarrama, 30TVL0603, $1.000 \mathrm{~m}, 26$. VI.1975, 1 q sobre hierba, V. J. Monserrat (VM), 12.VII.1976, $1.100 \mathrm{~m}, 1$ \& sobre Lavandula sp., J. J. Presa (VM), 24.II.1976, 1 larva en hojarasca de C. ladanifer, R. Outerelo (VM). Madrid, 1 o, R. Flores (VM), 1 +, G. Mercet (MNCN), La Moraleja, 30TVK4784, 650 m, 22.VI.1982, 1 q, M. Candela (MNCN), Puerta de Hierro, 30TVK7938, 20.V.1982, 1 ㅇ, M. Candela (MNCN), 24.V.1985, 2 우으. M. Candela (MNCN), Prádena del Rincón, 30TVL5444, 1.104 m, 24.VI.1987, 1 đ sobre brezo, 11.VI.1988, 1 ㅇ sobre Genista sp., 18.V.1989, 1 ते sobre brezo 22.VII.1989, 1 ㅇ sobre brezo, F. Marín (VM), Puerto de la Cruz Verde, 30TUK9790, 1.600 m, VII.1975, 1 đ̊, V. J. Monserrat (VM), Puerto de los Leones, Vía Crucis, 30TVL0305, 1.600 m, 2.X.1975, 1 larva sobre Cistus sp., R. Outerelo (VM), Teleférico, 30TVK3674, 650 m, 1.VI.1975, 1 $\widehat{\lambda}$, M. Candela (MNCN), Rivas Vaciamadrid, Montarco, 30TVK56, 590 m, 1.VI.2006, 1 đ̃, J.I. López Colón (VM), San Fernando de Henares, 30TVK5475, 585 m, 24.V.2008, 1 ㅇ, 14.V.2010, 1 đ̃, J.I. López Colón (VM), Torrejón de Ardoz, 30TVK5979, 585 m, 4.V.2008, 1 sobre Tamarix gallica, 20.V.2008, 1 q sobre T. gallica, J.I. López Colón (VM), Villalba, 30TVK1699, 1.108 m, 12.IV.1980, 1 † sobre Cistus sp., P. Garrumi (VM). Málaga: Benadalid, 30STF9754, $690 \mathrm{~m}$, 1.VI.1990, 1 đิ sobre Q. rotundifolia, 1.VI.1990, A. Baz (VM), Estepona, 30SUF0733, 21 m, 31.V.1989, 1 ô sobre Adenocarpus decorticans, A. Baz (VM), Las Cañillas, 30STF74, 25.V.2012, 1 ठ̊ sobre P. lentiscus, V. J. Monserrat (VM), Ronda, Sierra de las Nieves, 30SUF1964, 21.VI.1997, 1 §, J. De Ferrer (MNCN), Vélez, 30SVF07, 140 m, 11.V.2009, 1 $\widehat{\partial}$ en trampa de intersección, J.M. Vela (VM), Villanueva del Rosario, 30SUF7895, 697 m, 14.V.1988, 3 우, M. A. Zarazaga (MNCN). Murcia: Cehegín, 30SXH0417, 560 m, 10.VI.1982, 1 \& sobre Dorycnium pentaphyllum, V. J. Monserrat (VM), Sierra de Espuña, 30SXG29, 20.VI.1973, 1 Ĵ, leg.? (BM). Salamanca: Hinojosa del Duero, 29TPF8539, $601 \mathrm{~m}, 19 . \mathrm{IV} .1980,1$ ô, J. Pedrero (VM), La Madroñera, 29TPF9417, 700 m, 28.VI.1980, 2 đิ ô sobre Q. ilex, V. J. Monserrat (VM), Lumbrales, 29TPF9234, 673 m, 31.V.09, 1 †, sobre Q. pyrenaica, V. J. Monserrat (VM), Valdecarpinteros, 29TQF1604, 800 m, 30.V.1983, 1 q sobre Q. faginea, L. S. Subías, Fernández \& Berzosa (VM), 30.VI.1983, 1 § /, 1 †, L. S. Subías, Fernández \& Berzosa (VM). Segovia: Chañé, 30TUL8077, 767 m, 8.V.-3.VII.2000, 1 ऊ/2 2 ㅇ en trampa de Malaise, J. F. Gómez (VM), El Espinar, 30TUL9408, 1.191 m, 3.VII.1984, hojarasca de pino+musgo, 1 larva, R. Outerelo (VM). Soria: Puerto del Mojón Pardo, 30TWM0230, 1.234 m, 17.VI.1989, 1 \& sobre P. sylvestris, 
F. Marín (VM). Toledo: Mora, 30SVJ3393, 740 m, 14.V.1978, 1 ㅇ sobre O. europaea, V. J. Monserrat (VM), Sierra de Pelahustán, 30TUK6348, 4.VI.1978, $700 \mathrm{~m}, 1$ \& sobre J. communis, V. J. Monserrat (VM). Zaragoza: Mequinenza, 31TBF7484, $90 \mathrm{~m}$,

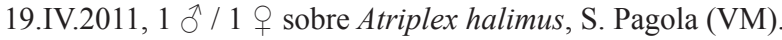

Harraphidia laufferi es una especie monotípica que no presenta problemas diferenciales por sus marcadas diferencias morfológicas, tanto en el $\widehat{\partial}$ como en la ㅇ.

MORFOLOGÍA EXTERNA: Es una especie de tamaño pequeño (figs. 2-4). El clípeo y el labro son pardos. Es escapo es pardo oscuro y tanto el pedicelo como el flagelo son pardos oscuro. El pronoto en su parte anterior es pardo y en la posterior negro con un dibujo que puede ir desde el amarillo al pardo, el pronoto se encuentra delimitado por una banda amarilla-pardusca (figs. 2-4, 9-11). En los apéndices locomotores las coxas II y III son negras y los fémures III son pardos. La coxa I, y los fémures I y II son pardos. El resto de los segmentos son amarillo-parduscos. La longitud del ala anterior es de entre 5.5-7.8 $\mathrm{mm}$ en los

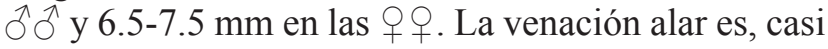
en su totalidad, parda, pudiendo aparecer en la base del ala venas o parte de venas de coloración ocre. El ala anterior no presenta una vena transversal suplementaria entre la C y el R (fig. 31). En el abdomen la coloración de los terguitos va desde pardo al negro, con una banda media amarillo-ocre. Los esternitos son pardos con manchas laterales y medianas amarillas (figs. 2-4).

SEGMENTOS GENITALES: En los $\widehat{\partial} \widehat{\partial}$ los escleritos de la hipovalva, en su zona media, presentan un proceso medio triangular (figs. 16, 61, 62), en su parte final se ensanchan a modo de espátula, convergiendo en su región caudal. El endofalo es muy evidente y el hipandrio interno es grande (figs.16, 61, 62, 86).

En las $\circ$ ㅇ el intersegmento $\mathrm{S} 7 / 8$ es corto, membranoso con pequeñas arrugas (figs. 17, 109). El ductus receptaculi surge del extremo anterior del sáculo (fig. 131), es muy largo y forma un ovillo. El receptáculo seminal es helicoidal, bien esclerificado y las glándulas anejas finalizan en un pequeño ensanchamiento (fig. 131).

BIOLOGÍA: Parece que el desarrollo transcurre en el sustrato edáfico. Existen numerosas capturas de imagos en sotobosque, tanto sobre fanerófitos, como nanofanerófitos, sobre todo acebuches y pinares abiertos (especialmente en pinos y enebros jóvenes), aunque ofrece una amplia gama de substratos vegetales, incluso en setos de jardines y puntualmente sobre hierba. Los biotopos son mediterráneos de tendencia xeroterma. La distribución vertical comprende desde el nivel del mar a los $1.600 \mathrm{~m}$. Se ha observado que esta especie muestra evidentes signos de tener capacidad de agregación, ya que en ocasiones

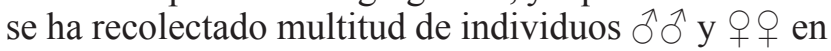

terminadas zonas de umbría en sotobosques, donde en un volumen de 1-2 $\mathrm{m}^{3}$ de vegetación, han sido observados docenas de individuos. En situaciones de estrés, como en la captura de ejemplares y alojamiento temporal de individuos vivos en espacios reducidos, se ha observado la existencia de canibalismo (Monserrat \& Papenberg, 2006).

FENOLOGÍA: Presenta una extensa distribución anual en la aparición de los imagos, dada su amplia y variada versatilidad ecológica, y dependiendo de la latitud y altitud. Existen registros en los meses (III) IV-VII.

\section{VARIABILIDAD: Escasa y sin valor taxonómico.}

ESTADIOS PREIMAGINALES: La larva se diferencia de la larva de Harraphidia (Harraphidia) harpyia por la existencia de una mancha aislada dentro de la banda lateral (fig. 149). A partir de crías ex ovo (no finalizadas) se han registrado periodos de desarrollo al menos de 2 años (H. Aspöck et al., 1991). Se han hallado larvas en hojarasca de alcornoques, jaras, pinos y musgos. Morfología y aspecto de la larva en figura 149 y en H. Aspöck et al. (1991).

DISTRIBUCIÓN: Centro y sur de España y Portugal (fig. 163). Elemento faunístico ibérico estacionario, monocéntrico e ibérico, probablemente de carácter atlantomediterráneo, aunque se sospecha que su distribución se limita exclusivamente a la Península Ibérica. La frontera septentrional de su distribución no está claramente delimitada, pero el número de registros en el tercio septentrional es, de momento, nulo (fig. 163), lo que se podría justificar por su carácter xerotermo.

DISCUSIÓN: Por su pequeño tamaño esta especie se suele reconocer con facilidad. Individuos pequeños de Atlantoraphidia maculicollis pueden ser muy parecidos, sobre todo porque las estructuras genitales del $\hat{o}$ son muy similares, pero $H$. laufferi carece del saliente en forma de espina del gonocoxito (figs. 57, $58,61,62)$. Respecto a la larva se diferencia de la de $H$. harpyia por la presencia de una mancha aislada dentro de la banda lateral (fig. 149). Esta especie ha sido citada junto a Subilla aliena, Atlantoraphidia maculicollis, Hispanoraphidia castellana, Ohmella bolivari, Ohmella baetica, Ohmella libidinosa, Fibla (Fibla) hesperica. En algunas localidades de la Península Ibérica es simpátrida de Harraphidia (H.) subdesertica.

Género Hispanoraphidia H. Aspöck \& U. Aspöck, 1968

Hispanoraphidia H. Aspöck \& U. Aspöck, 1968b: 61 (d, a, mf, tx), [especie tipo por designación original: Raphidia castellana Navás, 1915].

Considerado como subgénero de Raphidia Linnaeus, 1758: H. Aspöck \& U. Aspöck, 1968b: 61 (d, a, mf, tx). H. Aspöck et al., 1975: 371 (1). H. Aspöck et al., 1980a: vol. I: 86 (rf), 87 (dis), 118 (bio, dis, tx), vol. II: 253 (dis). 
Considerado como género: H. Aspöck, 1986: 25, 26 (dis). H. Aspöck et al., 1989: 89 (dis). H. Aspöck, 1990: 54, 56 (dis). H. Aspöck et al., 1991: vol. I: 54, 76, 354 (bio), 111, 115, 354 (tx), 353 (a), 354 (ô, , 1), 354, 555, 559 (dis), vol. II: 424 (dis). Oswald \& Penny, 1991: 64 (lst). U. Aspöck \& H. Aspöck, 1994: 39 (tx). H. Aspöck \& Hölzel, 1996: 38, 44 (dis). H. Aspöck, 1998b: 39 (tx). H. Aspöck et al., 2001: 19, 51 (tx), 51 (dis). H. Aspöck, 2002: 38, 45 (1), 45 (pa). H. Aspöck \& U. Aspöck, 2007: 99 (dis). Monserrat \& Papenberg, 2010: 63 (rf). Haring et al., 2011: 3 (dis), 12 (tx), fig.S3 (dis). U. Aspöck et al., 2012: 577 (lst), 577, 579, 580 (f). H. Aspöck \& U. Aspöck, 2013: 12 (tx), 40 (gen).

Navás describió en el año 1915 la especie Raphidia castellana para la cual H. Aspöck \& U. Aspöck (1968b) crearon el subgénero Hispanoraphidia, que posteriormente fue elevado a rango de género. Es un género monotípico endémico de la Península Ibérica (fig. 164), cuya única especie es Hispanoraphidia castellana.

\section{Hispanoraphidia castellana (Navás, 1915)}

Raphidia castellana Navás, 1915: Navás, 1915a: 793 (d), 793, 794, (mf), 793, 794, 797 (a). Navás, 1915b: 875 (dis). Marín \& Monserrat, 1987: 351, 353, 354, 356 (bio). Marín \& Monserrat, 1991: 190 (bio), 192 (dis). Marín, 1994: 254, 255, 260-262 (bio). Monserrat \& Triviño, 2013: 15 (lst).

Raphidilla castellana (Navás, 1915): Navás, 1919: 51 (c), 67 (a, dis, mf, §̋). Navás, 1923: 72 (c, dis). Lestage, 1928: 32 (a). Navás, 1928a: 22 (c), 38 (ङึ), 38, 39 (a, mf), 39 (dis). Navás, 1933a: 121 (lst). Navás, 1934a: 6 (dis). Navás, 1934b: 32 (c), 46 (a, ô, o ), 46, 47 (mf), 47 (dis). Steinmann, 1964: 216 (a), 217 (c). Monserrat \& Triviño, 2013: 15 (lst).

Raphidia (Hispanoraphidia) castellana Navás, 1915: H. Aspöck \& U. Aspöck, 1968b: 61 (a, ô, O, mf, tx). H. Aspöck et al., 1980a: vol. I: 39 (c), 118 (a, bio, 1, mf, tx), 118, 351 (dis), vol. II: 36 (ठ) ), 41 (), 166 (a), 253 (dis). Díaz-Aranda et al., 1986: 1.134 (dis). Monserrat \& Díaz-Aranda, 1987: 175 (dis). DíazAranda \& Monserrat, 1988a: 218 (dis). Monserrat \& Triviño, 2013: 15 (lst).

Hispanoraphidia castellana (Navás, 1915): H. Aspöck et al., 1985a: 387 (pa). H. Aspöck et al., 1991: vol. I: 89, 355 (bio), 96, 100, 356 (pa), 129, 356, 550, 551, 576, 577 (dis), 130 (c), 354-356 (fe), 356 (p), 614 (1st), vol. II: 52 (mf), 75 (a), 175 (ô), 271(†), 355 (1), 487 (dis). Marín \& Monserrat, 1995: 112 (bio, dis). H. Aspöck \& Hölzel, 1996: 44 (dis). Monserrat, 1996: 12 (1st). H. Aspöck et al., 2001: 51 (tx), 51, 332, 338 (dis). Haring et al., 2011: 4 (dis), 10 (tx), fig.S2 (f). H. Aspöck \& U. Aspöck, 2013: 33 (lst), 54 (d, ô, p), 48 (gen), 85, 86 (eco). Monserrat, 2013: 287 (1st). Monserrat \& Triviño, 2013: 15, 40 (dis, lst, tx).

Raphidia hispanica Rambur, 1842: Navás, 1915b: 861, 863, 871, 874 (dis), 862 (mf, đ) ), 862, 863 (a) - ie.

Raphidilla hispanica Rambur, 1842: Navás, 1919: 51 (c, ie), 66 (a, dis, mf, đ) - ie. Navás, 1920: 43 (dis) - ie. Navás, 1928a: 22 (c), 36, 37 (mf), 37 (a, ठ) - ie. Navás, 1933a: 121 (lst) - ie. Navás, 1934a: 6 (dis) - ie. Navás, 1934b: 45 (a, mf, ô, 우), 32 (c), 46 (dis) - ie.

TIPO: lectotipo $\widehat{\partial}$ depositado en el Museo Municipal de Barcelona. LOCALIDAD TÍPICA: El Escorial, Madrid (España).

MATERIAL ESTUDIADO: ESPAÑA: Albacete: El Ballestero, 30SWH5297, 950 m, 7.VI.1991, 1 ㅇ sobre Quercus ilex, F. Marín (VM), Cortijo de las Tortas, 30SWH5169, 1.420 m, 8.VI.1991, 2 우 sobre Pinus halepensis, F. Marín (VM), La Hoz, 30SWH4802,
1.100 m, 23.VI.1990, 1 q sobre Quercus sp., F. Marín (VM). Ávila: Arevalillo, 30TTK9995, 1.130 m, 14.VI.1977, 1 ㅇ sobre Pinus sp., V. J. Monserrat (VM), Ávila (Provincia), 5.VI.1920, 1 ㅇ, J. Bajo (MNCN), Bohoyo cruce crta. Barco, 30TTK9367, 1.150 m, 2.VI.08, 1 ㅇ en prado M. Portillo (USAL), Collado La Mina, 30TVL0104, $1.711 \mathrm{~m}, 16$. VII.1975, 1 ô, V. J. Monserrat (VM), Hoyos del Espino, Sierra de Gredos, 30TUK1569, 1.440 m, 28.VI.1971, 2 qㅇ, E. Serrano (VM), 27.VI.1985, 1 +, J.L. Viejo \& J. Martín Cano (VM), 8.VII.1987, 1 , E. Serrano (NHM), Piedralaves, Garganta de Nuñocojo, 30TUK5564, $621 \mathrm{~m}$, 3.V.1975, 1 q sobre Q. ilex, V. J. Monserrat (VM), Puerto del Pico, 30TUK2965, 1.391 m, VI.1906, 1 , L. Navás (MNCN), Puerto del Tremedal, 30TTK7770, 1.637 m, 17.V.1980, 1 ठ̊ en estómago de Podarcis hispanica, V. Pérez-Mellado (VM), Sierra de Gredos (Parador de Gredos), 30TUK2069, 1.600 m, 29.IV.1977, 1 ㅇ sobre P. sylvestris, V. J. Monserrat (VM). Cádiz: Sierra del Pinar, 30STF8371, 1.100 m, 24.V.1977, 2 đ̊ ô sobre Q. canariensis, V. J. Monserrat (VM). Cáceres: Castaña de Ibor, 30STJ9289, $650 \mathrm{~m}$, 8.VI.1980, 1 시 2 우 sobre Castanea sativa, V. J. Monserrat (VM), El Torno, 30TTK4847, 769 m, 4.V.1982, 1 §̂, C. Urones (VM). Ciudad Real: Navas de Estena, 30SUJ6972, 675 m, 12.V.1991, 1 , , Ortuño \& Costas (VM), 7.VI.2012, 1 q sobre $Q$. ilex ballota, F. Acevedo (VM). Cuenca: Beteta, 30TWK7892, 1.200 m, 30.VI.1985, 1 §े sobre Salix sp., V. J. Monserrat (VM), Cuenca, 30TWK7436, 1.000 m, 28.V.1986, 1 đ sobre Q. ilex, A. Baz (VM), Masegosa, 30TWK8289, 1.390 m, 26.V.1985, 1 q sobre Crataegus monogyna, V. J. Monserrat (VM), Naharros, 30TWK4134, 939 m 14.VI.1979, 1 o sobre Q.ilex, C.P. Iñigo (VM), Tragacete, 30TWK9767, 1.269 m, 28.V.1986, 1 ㅇ sobre Crataegus, A. Baz (VM), Uña, 30TWK8753, 1.146 m, 28.V.1986, 1 q sobre Q. faginea, A. Baz (VM), Villar de Olalla, 30TWK6829, 900 m, 25.V.1985, 1 sobre Q. faginea, V. J. Monserrat (VM). Girona: Estela, 31TDG7581, 460 m, 23.V.1966, 2 ㅇ, J. Ábrahám (NHM). Granada: Collado de la Sabina, Sierra Nevada, 30SVG6208, 2.250 m, 30.VI.2011, 3 취 / 3 우 sobre $P$. sylvestris, V. J. Monserrat (VM), 1 ○े sobre P. sylvestris, F. Acevedo \& V. Triviño (VM), 10.VII.2012, 4 ええ/ 8 우 sobre P. sylvestris, V. J. Monserrat (VM), 10.VII.2013, 16 ๙रे/11 우 sobre P. sylvestris, V. J. Monserrat (VM), 27.VII.2013, 6 주 / 5 우 0 sobre P. sylvestris, V. J. Monserrat (VM), 18.VII.2014, 2 के के sobre P. sylvestris, V. J. Monserrat (VM), El Dornajo, 10.VII.2013, 3 우 sobre $Q$. rotundifolia, V. J. Monserrat (VM), Fuente de Cortichuela, 30SVG5904, 1.900 m, 3.VII.1986, 1 ô sobre Q. ilex, 1 ô sobre P. sylvestris, leg.? (VM), Hoya de la Mora, Sierra Navada, 30SVG5825, 2.500 m, 30.VI.2011, 1 sobre P. sylvestris, F. Acevedo, V. Triviño (VM), Puerto de Onitar, 30SVG4544, 900 m, 1.VI.1986, 1 o sobre Q. ilex, leg.? (VM), Puerto de Trevenque, 30SVG5703, 2.000 m, 3.VII.1986, 1 ઈ/ 3 우 sobre P. sylvestris, leg.? (VM). Guadalajara: Alcoroches, 30TXK0698,

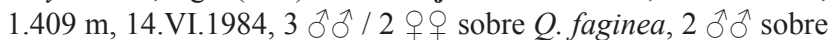
P. sylvestris, V. J. Monserrat (VM), Aragoncillo, 30TWL8130, $1.200 \mathrm{~m}, 14 . \mathrm{VI} .1984,1 \hat{\jmath}\left({ }^{\star} *\right) / 2$ 우 0 sobre Juniperus thurifera, V. J. Monserrat (VM), Cantalojas, Tejera Negra, 30TVL698647, 1.620 m, 26.VI.1988, 1 § sobre Fagus, 1 क sobre Q. pyrenaica, F. Marín (VM), Cardeñosa, 30TWL1251, 1.074 m, 30.VI.1984, 1 ㅇ sobre Q. ilex, J. M. Díaz (VM), Chiloeches, 30TWK8691, 785 m, 24.V.1988, 1 q sobre Q. rotundifolia, V. J. Monserrat (VM), Durón, 30TWK2397, 755 m, 7.VI.1992, 1 đે, Munguira (UAM), El Recuenco, 30TWK5598, 978 m, 15.VI.1984, 1 ô sobre Q. ilex, V. J. Monserrat (VM), 5.VII.1984, 1 §, V. J. Monserrat (VM), Gajanejos, 30TWL0820, 1.028 m, 14.VI.1984, 4 ふ઼へ / 1 ㅇ sobre Q. ilex, V. J. Monserrat (VM). Luzón, 30TWL6044, 1.176 m,

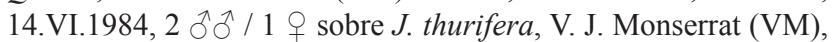
5.VII.1984, 1 +, L. M. Díaz-Aranda (VM), Mandayona, 30TWL2133, 900 m, 30.VI.1984, 1 q sobre Q. ilex, F. Marín 
(VM), Mirabueno, 30TWK1683, 1.064 m, 11.VI.1984, 1 ô, sobre Q. ilex, 21.VI.1984, 1 \& sobre Q. ilex, F. Marín (VM), Ruguilla, 30TWL3511, 886 m, 4.VI.1972, 1 †, Silván (VM), Setiles,

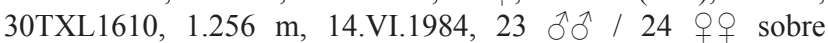

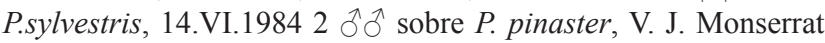
(VM), 5.VII.1984, 1 ô sobre Q. faginea, L. M. Díaz-Aranda (VM), Tamajón, 30TVL7939, 1.029 m, 29.V.198, 1 ô, sobre Cistus laurifolius, V. J. Monserrat (VM), Taravilla, 30TWL8705, 1.317 m, 15.VI.1984, 1 ㅇ, 6.VII.1984, 2 우 sobre $Q$. faginea, V. J. Monserrat (VM), Traid, 30TXL0003, 1.376 m, 15.VI.1984, 2 우우 sobre J. phoenicea, V. J. Monserrat (VM), Valdeaveruelo, 30TVK7398, 738 m, 26.V.1984, 1 §ै sobre Q. ilex, V. J. Monserrat (VM), Valdepinillos, 30TVL8557, 1.380 m, 27.V.1989, 1 ô sobre P.sylvestris, F. Marín (VM), Valverde de los Arroyos, 30TVL8053, $1.250 \mathrm{~m}, 29 . \mathrm{V} .1988,1$ ๙ / 1 q sobre Q. rotundifolia, 2.VI.1989, 1 đo sobre brezo, F. Marín (VM), Villaverde y Paraconsol, 30TWL4239, 800 m, 25.V.1985, 1 ㅇ sobre Q. ilex, V. J. Monserrat (VM). Jaén: Collado de los Jardines, 30SVH5648, $742 \mathrm{~m}$, 20.VI.2004, 1 §ै sobre Cistus ladanifer, V. J. Monserrat (VM), Sierra de Cazorla, Cantalar, 30TWG0097, 800 m, 5.IV.1975, 1 శ sobre Q. faginea, L. S. Subías (VM). Sierra de Cazorla, Camino de Agracea, 30SWH1849, 12.VI.1991, 1 † sobre Rosa pruzini, 14.VI.1991, 1 †, 16.VI.1991, 1 ๆ, J. L. Yela (VM), Sierra de Cazorla, Nacimiento del Guadalquivir, 30SWG0385, $1.400 \mathrm{~m}$, 2.VII.1978, 1 +, R. Outerelo (VM), Sierra de Cazorla, Roblehondo, 30SWG0899, 1.300 m, 17.V.1991, 1 ㅇ, J. L. Yela (VM), Sierra Magina, 30SVG57, 1.V.2000, 1 đ̊ sobre O. europea, (EEZ). León: Barrios de la Luna, 30TN6647, 1.039 m, 13.VIII.1996, 1 , A.F. \& R.F. (UNIL), Carbajal de la Legua, 30TTN8626, $992 \mathrm{~m}$, 31.V.1994, 1 9, J. Alberto (UNIL), Pedrún de Torio, 30TTN9540, 990 m, 3.IX.1990. 1 ㅇ, C. García Selz (UNIL). Madrid: Alcalá de Henares, 30TVK6981, 688 m, 29.V.1985, 1 q sobre hierba, V. J. Monserrat (VM), Arroyo de Navalmedio, 30TVL1212, $1.300 \mathrm{~m}$, 28.VI.1975, 2 우, ․ V. J. Monserrat \& J. Mateos (VM), Arroyo del Rayo, Garganta de los Montes, 30TVL4228, 1.420 m, 29.V.1994, 1 ô, M.A. Alonso Zarazaga (MNCN), Cabeza Lijar, 30TVL0205, 1.700 m, 26.VI.1975, 1 ऽ / 1 ㅇ, V. J. Monserrat (VM), Casa de Campo, 30TVK3675, 650 m, 11.V.1968, 1 ․ R. Outerelo (VM), Cercedilla, 30TVL1010, $1.188 \mathrm{~m}, 2$ ઈิ่ / 2 우, C. Bolívar (MNCN), 1 , Navás (MNCN), VII, 1 , E. Morales (MNCN), 5.VII.1960, 1 ㅇ, S. V. Peris (VM), Cerro Colgado, 3.VII.1983, 1 , leg.? (VM), Colmenar Viejo, 30TVL3409, 883 m, 28.V.2005, en vuelo, 1 ․, N. del Palacio (VM), El Escorial, 30TVK0293, 909 m, 24.V.1987, 1 ふૈ, leg.? (VM), El Escorial, La Herrería, 30TVK0292, 1.000 m, 25.V.1975, 1 ते sobre Cardamine pratenis, 7.V.1976, 1 o, V. J. Monserrat (VM), El Escorial, Monte Abantos, 1.750 m, 26.VI.1977, en Piornal-Pinar, 2 $\widehat{\jmath} / 2$ + , R. Outerelo (VM), El Escorial, Silla Felipe II, 30TVK0291, 1.089 m, 7.V.1976, en vuelo, 1 $\widehat{\jmath}$, V. J. Monserrat (VM), El Escorial, Fte. del Cervunal, Abantos, 30TVK0296, 1.700 m, 8.VIII.2007, 4 q ㅇ sobre $P$. syl-

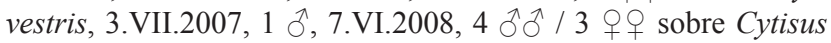

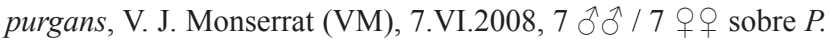
sylvestris, V. J. Monserrat (VM), 21.VI.2008, 4 + sobre $P$. sylves-

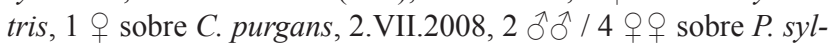
vestris, V. J. Monserrat (VM), El Ventorrillo, 30TVL1312, 1.500 m, 21.VII.1975, 1 ㅇ, V. J. Monserrat (VM), 1.480 m, 23.V.1988, 2

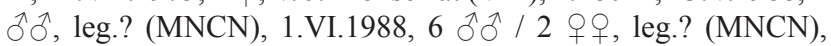
13.VI.1988, 1 § / 1 q, J. L. Nieves (MNCN), 1.480 m, 3.VII.1988, 1 o, leg.? (MNCN), Real Sanatorio de Guadarrama, $1.480 \mathrm{~m}$, 7.VI.1977, 1 q sobre Pinus, J. J. Presa (VM), Guadarrama, 30TVL0803, 1959, 1 , S. V. Peris (VM), Hoyo de Manzanares, 30TVK2397, $950 \mathrm{~m}, 13 . \mathrm{V} .1976,2$ ऊิ ठे sobre Q. ilex, V. J. Monserrat (VM), La Cabrera, 30TVL4824, 1.100 m, 13.VI.1975, 1 , V. J. Monserrat (VM), 23.IV.1976, hojarasca de encina-roble, larva, R. Outerelo (VM), La Hiruela, 30TVL6147, 1.300 m,
12.VI.1984, 1 q sobre Erica arborea, A. Baz (VM), La Navata, 30TVK19, 880 m, 4.III.1976, 1 larva en hojarasca de C. ladanifer y musgo, J. Berzosa (VM), 1 larva sobre hojarasca de encina, J. Berzosa (VM), Los Molinos, 30TVL0907, 1.045 m, 10.VI.1973, 1 q sobre Q. pyrenaica, R. Outerelo (VM), Madrid, 30TVK37, 650 m, 1 +, Bolívar (MNCN), Miraflores de la Sierra, 30TVL3518, $1.150 \mathrm{~m}, 19 . \mathrm{VI} .1988,1$ ㅇ sobre Q. pyrenaica, V. J. Monserrat, (VM), Montejo de la Sierra, 30TVL5545, 1.152 m, 10.VI.1986, 1 \&, E. Plaza, (MNCN), Navacerrada, 30TVL1409, 1.203 m, 27.VI.1971, 1 ô, R. Outerelo (VM), 17.VIII.1978, 1 larva sobre Arctostaphylos uva-ursi, J. Berzosa (VM), Puerto de Cotos, 30TVL1919, 1.840 m, 14.VII.1975, 1 ô, V. J. Monserrat (VM), 21.VII.1975, 1 Jै, V. J. Monserrat (VM), Puerto de Malagón, 30TUL9000, $1.530 \mathrm{~m}, 31 . I I I .1976,1$ larva sobre Cytisus, R. Outerelo (VM), $1.680 \mathrm{~m}, 23 . \mathrm{V} .1976,1$ ㅇ sobre P. sylvestris, V. J. Monserrat (VM), Puerto de la Morcuera, 30TVL2920, $1.796 \mathrm{~m}$, VI.1926, 1 ㅇ, F. Bonet (MNCN), 1.600 m, 4.VI.1975, 2 ठิ ठิ / 3

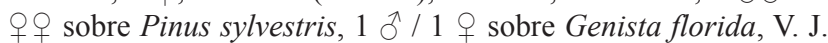
Monserrat (VM), 1.400 m, 27.VI.1975, 1 q, sobre Cytisus, V. J. Monserrat (VM), $1.300 \mathrm{~m}, 12 . \mathrm{II} .1976$, pino y musgo, larva, R. Outerelo (VM), Puerto de los Leones, Puerto de Guadarrama, 30TVL0307, 1.511 m, 13.VII.1976, pinar, 1 q, J. J. Presa (VM), Vía Crucis, 30TVL0305, 1.600 m, 4.V.1976, 1 larva sobre Cistus laurifolius, R. Outerelo (VM), Puerto de Majalasna, 30TVL1016, 1.933 m, 17.VIII.1978, 1 larva sobre Pinus, 1 larva sobre Cytisus sp., J. Berzosa (VM), Puerto de Navafría, 30TVL3137, 1.700 m, 30.VI.1977, prado-pinar, 1 q, J. J. Presa (VM), Arroyo del Palomar, 30.VI.1977, prado-pinar, 1 +, J. J. Presa (VM), Puerto de Peñaquemada, 30TVL4248, $1.760 \mathrm{~m}$, 28.VII.1975, piornal-pinar, 1 q, V. J. Monserrat (VM), Rascafría, 30TVL2528, $1.163 \mathrm{~m}$ 31.V.1976, sobre umbelífera, 1 ㅇ, V. J. Monserrat (VM), El Paular, 30TVL2527, 6.VI.1976, 1 J, V. J. Monserrat (VM), San Fernando de Henares, 30TVK5475, 585 m, 14.V.2010, 1 q, J.I. López Colón (VM), Soto del Real, 30TVL3312, $921 \mathrm{~m}, 17 . \mathrm{VI} .1993,1$, Munguira (UAM), Tablada, 30TVL0506, 981 m, 16.VII.1975, 1 ㅇ sobre Retama sp. (flor), V. J. Monserrat (VM), 18.VI.1976, 1 ㅇ sobre $P$. sylvestris, R. Outerelo (VM), Torres de Alameda, 30TVK6973, 654 m, 11.VI.1984, 1 ㅇ sobre Q. faginea, A. Baz (VM), Valle de Fuenfria, 30TVL1016, 1.188 m, 1 ภे, J. Álvarez, (MNCN), Villalbilla, 30TVK7475, 747 m, 27.VII.1984, piscina, 1 ㅇ, V. J. Monserrat (VM). Orense: Esgos, 29TPG0786, $600 \mathrm{~m}$, 25.VI.1977, 1 \& sobre P. pinaster, V. J. Monserrat (VM). Pontevedra: Villagarcía de Arosa, 29TNH1916, 55 m, 1947, 1 \&, J. L. Saavedra (VM). Salamanca: Candelario, 30TTK6772, 1.200 m, 13.V.1977, 1 + sobre P. sylvestris, V. J. Monserrat (VM), Caño de Sierra Lumbrales, 29TPF9234, 6.V.1977, 1 ô sobre Q. ilex, F. Cabezas (VM), Ermita del Cueto, 30TTL5823, 780 m, 25.IV.1977, 3 ô $\hat{\jmath}$ sobre Crataegus monogyna, V. J. Monserrat (VM), Fuente de Linares, Linares de Riofrío, 30TTK5296, 1.000 m, 13.V.1977, 1 ㄱ/ 2 우 sobre C. scoparius, V. J. Monserrat (VM), Los Caozos, Picón del Palo, 29TQF17, 600 m, 7.V.1977, 2 우, V. J. Monserrat (VM), Milano, 29TQF0151, 750 m, 7.V.1977, 3 đ̂ ổ sobre C. multiflorus, V. J. Monserrat (VM), 1 đ sobre Q. pyrenaica, V. J. Monserrat (VM), Minas de Barruecopardo, 29TPF9649, $730 \mathrm{~m}$, 6.V.1977, 1 ô sobre Cytisus, F. Cabezas (VM), Navasfrías,

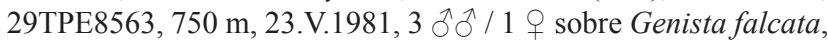
1 ㅇ sobre Pteridium aquilinum, C. Urones (VM), Pereña,

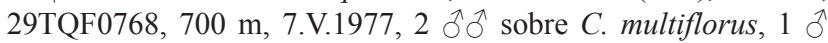
sobre Q. pyrenaica, 1 + sobre C. scoparius, V. J. Monserrat (VM), Puerto de las Mezas, 29TPE8260, 1.265 m, 23.V.1981, 1 o sobre Erica arborea, C. Urones (VM), Sorihuela, 30TTK7380, 1.020 m, 13.V.1977, 1 q sobre Q. pyrenaica, V. J. Monserrat (VM), 20.V.1977, 1 oे sobre Q. pyrenaica, Cabezas (VM). Segovia: Aldeavieja, Puerto de la Lancha, Rio Tuerto, 30TUK8099, 1.400 m, 25.VI.1977, 9 ठึ ठ / 4 우 sobre C. purgans, R. Outerelo (VM), 
Boca del Asno, 30TVL12, 1.250 m, 27.V.1993, 1 ㅇ, Munguira (UAM), El Espinar, 30TUL9408, 1.191 m, 25.VI.1977, pradoroble-espinar, 1 + , R. Outerelo (VM), El Muyo, 30TVL7370,

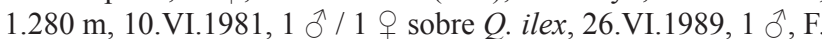
Marín (VM), La Granja de San Ildefonso, 30TVL1528, 1.193 m, VI.1908, 1 o, J. Sanz (VM), La Machorra, 1.600 m, 20.V.1976, 1 larva en hojarasca de pinar $J$. communis nana, $1.600 \mathrm{~m}$, leg.? (VM), Ortigosa, 30TVL0022, 1.166 m, 29.V.1955, 1 ㅇ, S. V. Peris (VM), Peñalara, Arroyo de los Accidentes, 30TVL2122, $1.700 \mathrm{~m}$, 28.VI.1977, 1 q sobre Pinus, J. J. Presa (VM), Riofrío de Riaza, Hayedo de la Pedrosa, 30TVL6266, 1.312 m, 18.VII.1992, 1 , V. Ortuño (VM). Teruel: Bañón, 30TXL5222, 1.040 m, 27.IV.1987, 1 đิ sobre Q. rotundifolia, V. J. Monserrat (VM), La Iglesuela del Cid, 30TYK2784, 1.320 m, 19.VI.1987, 1 q sobre P. sylvestris, V. J. Monserrat (VM), Puerto de Pozondón, 30TXK2589, 1.407 m, 1 ㅇ, E. Galante (VM). Toledo: Navamorcuende, El Piélago, 30TUK4846, 25.V.2011 1 q sobre P. sylvestris, V. J. Monserrat (VM), Sierra de Hermo, 30SUJ48, 820 m, 25.V.1978, 2 ๙ै ô sobre Q. faginea, V. J. Monserrat (VM). Valladolid: Bobadilla del Campo, 30TUL3063, $762 \mathrm{~m}, 21 . \mathrm{V} .1994,1$ o sobre $Q$. rotundifolia, V. J. Monserrat (VM), Tordesillas, 30TUL3396, $702 \mathrm{~m}$, 16.V.1982, 1 ठ, E. Galante (VM). Zamora: Barjacoba, 29TPG6965, 1.423 m, 18.VII.1977, 1 ㅇ, R. Outerelo, J. J. Presa, L. Subías (VM), Boya, Sierra de la Culebra, 29TQG1944, 878 m, 30.V.2004, 1 ते / 3 क, F. Novoa (VM), Puebla de Sanabria, 29TPG9558, 960 m, 14.V.1977, 1 ô sobre C. multiflorus, V. J. Monserrat (VM), San Pedro de Herrerías, 29TQG1742, 920 m, 8.V.1977, sobre P. sylvestris, 1 ते, V. J. Monserrat (VM), Valparaíso, 30TTL7180, $800 \mathrm{~m}, 15 . \mathrm{V} .1977,1$ đo sobre Q. ilex, I. Reviejo (VM), 10 ठำ / 4 우우 sobre C. scoparius, 3.VI.1977, 1 ¿ sobre $Q$. faginea, V. J. Monserrat (VM), Villadepera, 29TQG3905, $650 \mathrm{~m}, 8 . \mathrm{V} .1977,1$ sobre C. scoparius, V. J. Monserrat (VM).

Hispanoraphidia castellana es una especie muy característica por la morfología de su genitalia, tanto en el $\widehat{\partial}$ como en la $\rho$, y no presenta problemas de identificación.

MORFOLOGÍA EXTERNA: Es una especie de tamaño pequeño o mediano. Tanto en el clípeo como en el labro, su parte basal es de coloración parda y su parte apical de coloración parda oscura. En las antenas el escapo es pardo y el pedicelo pardo oscuro, así como el flagelo, que en su posición proximal es ocre y el resto es pardo oscuro. El pronoto es corto y delgado siendo su parte anterior parda y la caudal negra con un dibujo pardo claro. En los apéndices locomotores la coxa I y los fémures I, II y III son pardos, las coxas y trocánteres II y III son pardo oscuros y el resto de los artejos son ocres.

En el abdomen los terguitos y esternitos son de color pardo oscuro, teniendo ambos en el borde posterior una banda amarilla, que es más amplia en los esternitos. La longitud del ala anterior es de 7.5-9.2 $\mathrm{mm}$ en los $\widehat{\partial} \widehat{\partial}$ (fig. 32), y de 9-10.5 mm en las 우오. La venación alar es parda, existiendo en la región basal venas que parte de ellas tienen una coloración ocre. El pterostigma es amarillo, se encuentra atravesado por una vena y presenta tras él una vena apical (fig. 32). En el ápice alar, la parte distal del sector del radio tiene una vena bifurcada y otra sin bifurcar. La parte basal de la Ma del ala posterior tiene un recorrido transversal (fig. 32).

SEGMENTOS GENITALES: En los $\partial^{\lambda}$ el $8^{\circ}$ esternito se encuentra íntimamente ligado al $9^{\circ}$ esternito (fig. 64). El $9^{\circ}$ terguito se alarga dorsalmente, pero su cara lateral se estrecha y se encuentra unida al gonocoxito en un gran tramo, siendo el $9^{\circ}$ esternito muy delgado (figs. 64, 65). El gonocoxito se encuentra dividido por un apodema del estilo en una parte dorsal muy pequeña y una parte ventral grande cuya región dorsal es abombada y porta una prolongación orientada en dirección ventral (fig. 64). En el gonocoxito existe un proceso largo en forma de brazo (fig. 64). Los estilos son grandes y robustos con la base ensanchada y el extremo bifurcado con un ápice en dirección dorso-caudal y otro en dirección ventro-caudal. El ectoprocto es muy largo, siendo estrecho en su base y ensanchándose en su parte distal (fig. 63). La hipovalva es par, su base es ancha y muy divergente y los escleritos en su extremo están afilados y orientados en dirección proximal (fig. 65). Faltan los parámeros y no se delimita un gonarco. El hipandrio interno es muy delicado pero de gran tamaño (fig. 87).

En las 90 el $7^{\circ}$ esternito se prolonga en dirección caudal, siendo el borde posterior convexo (fig. 110). El intersegmento $\mathrm{S} 7 / 8$ es membranoso y profundo. Respecto a la genitalia interna, el atrio de la bolsa copuladora es compacto, está doblado y se encuentra más o menos esclerotizado (figs. 133, 134). El ductus saculi está representado por una pequeña estrangulación (fig. 134). El sáculo es muy membranoso, presentando una banda media dorsal característica (fig. 134). El ductus receptaculi parte de la porción media y tiene forma de tubo. El receptáculo seminal es piriforme con dos salientes en posición subterminal muy característicos. Las glándulas anejas del receptáculo seminal son filiformes (fig. 133).

BIOLOGÍA: La mayoría de los individuos han sido capturados sobre mesofanerófitos y macrofanerófitos de vegetación mediterránea en bosques esclerófilos (Quercus) o bosques aciculifolios (Pinus). También existen registros sobre vegetación arbustiva (Cytisus, Erica, Retama, Crataegus, etc.), a veces sobre flores e inflorescencias. Suelen evitar biotopos extremadamente templados y secos, y su distribución altitudinal global comprende entre los 55-2.500 m, apareciendo ejemplares cerca del piso alpino en la proximidad de pinares ricos en Berberis. Se ha observado cierta capacidad de agregación en los imagos, y como comentan Marín \& Monserrat (1991) las densidades de población suelen ser altas, incluso pudiendo aparecer de forma masiva.

FENOLOGÍA: Presenta una extensa distribución anual en la aparición de imagos dependiendo de la altitud y latitud. La actividad de los imagos es 
monovoltina, estacionaria, primaveral y estival transcurriendo durante los meses IV-VIII. (IX?)

ESTADIOS PREIMAGINALES: Las larvas son conocidas de la única especie del género Hispanoraphidia castellana. En los segmentos abdominales de la larva el dibujo dorsal alcanza el borde caudal (fig. 150). La banda media dorsal es delgada, estando interrumpida en el tercio cefálico por una zona pigmentada (fig. 150). La banda lateral, por lo general, transcurre por todo el segmento, pudiendo estar pigmentada de forma irregular, sobre todo en la parte anterior. La figura lateral sobrepasa algo la sutura dorsal presentando algunas pequeñas zonas más claras (fig. 150). La figura ventral ocupa escasamente la mitad del segmento con una banda media ancha. Se conoce la existencia de un parasitoide Meteorus punctifrons Thoms. (Hymenoptera, Braconidae) de una cita en Sierra Nevada (H. Aspöck et al., 1991). El desarrollo se realiza sobre el sustrato edáfico. Existen registros de larvas en el detritus de raíces de Berberis y Genista. Mediante cría ex ovo se han registrado tiempos de desarrollo de dos años, aunque por observaciones de crías de larvas recolectadas, parecen ser frecuentes desarrollos larvarios de tres años. La última hibernación transcurre en fase de pupa, y por tanto es un ciclo vital de tipo II (fig. 18) pudiendo solo excepcionalmente invernar en fase de larva (ciclo vital tipo I). El estadio de pupa se mantiene entre 7-10 meses. Si la pupación ocurre durante la primavera se prolonga pocas semanas. Morfología y aspecto de la larva en figura 150 y en H. Aspöck et al. (1991).

VARIABILIDAD: La variabilidad morfológica dentro de la especie es escasa. Existen algunos individuos de coloración oscura. En las $q$,, siendo el $8^{\circ}$ terguito largo y estrecho alcanzando ampliamente la región ventral, existen ejemplares que presentan en algunos casos un saliente dirigido hacia el espiráculo.

DISTRIBUCIÓN: España y Portugal. Elemento faunístico estacionario, monocéntrico e ibérico de carácter atlantomediterráneo. Citado en gran parte de la zona centro y norte de la Península Ibérica así como en cotas altas del Sistema Penibético, justificado probablemente al evitar biotopos extremadamente templados y secos (fig. 164).

DISCUSIÓN: De las especies que aparecen de forma simpátrida junto a $H$. castellana son Atlantoraphidia maculicollis y Harraphidia laufferi las que presentan un cierto parecido morfológico al tener los estilos bifurcados (figs. 57, 58, 61-65). Hispanoraphidia castellana se diferencia de Atlantoraphidia maculicollis al presentar el estilo una base ensanchada y tener el proceso del gonocoxito forma de brazo (fig. 64), al contrario que esta última, en donde el estilo carece de esa base ensanchada y el proceso del gonocoxito tiene forma de espina (fig. 57). Harraphidia laufferi carece de proceso en el gonocoxito (fig. 61). No se conoce con seguridad el grupo hermano del género Hispanoraphidia (H. Aspöck et al., 2001). La bifurcación de los estilos en los $\widehat{\partial} \widehat{\partial}$ y la forma de la bolsa copuladora en las $ㅇ$ se podría interpretar como sinapomorfías con el género Atlantoraphidia y Harraphidia. Finalmente puede que el proceso de los gonocoxitos largo en los $\partial \widehat{\partial}$ y el $8^{\circ}$ terguito que alcanza ampliamente la zona ventral en las $q$ ㅇ se pueda interpretar como caracteres sinapomórficos entre Hispanoraphidia y Africoraphidia. Esto significa que la bifurcación de los estilos ha podido surgir de forma independiente en Atlantoraphidia y Harraphidia. La base ampliamente divergente de la hipovalva y la pérdida del gonarco y parámeros son ambos caracteres autapomórficos de Hispanoraphidia. Por otro lado, los salientes subterminales del receptáculo seminal en la $q 9$ pueden indicar la existencia de sinapomorfías con el género Ohmella. Todos los géneros mencionados forman probablemente un grupo monofilético según H. Aspöck et al. (1991).

Especies simpátridas de Hispanoraphidia castellana han sido citadas Subilla aliena, Atlantoraphidia maculicollis, Harraphidia laufferi, Ohmella bolivari, Ohmella libidinosa, Ohmella casta y Fibla (Fibla) hesperica.

Género Ohmella H. Aspöck \& U. Aspöck, 1968

Ohmella H. Aspöck \& U. Aspöck, 1968b: 62 (d, a, ô, ๆ, mf), [especie tipo por designación original: Agulla voluptaria H. Aspöck \& U. Aspöck, 1968].

Considerado como subgénero Raphidia Linnaeus, 1758: H. Aspöck \& U. Aspöck, 1968b: 62 (d, a, đ̂, †, mf). H. Aspöck \& U. Aspöck, 1971c: 25 (rf), 30-32 (dis). H. Aspöck et al., 1975: 372 (1). H. Aspöck et al., 1980a: vol. I: 86 (tx), 87 (dis), 117 (a, rf), 118 (rf), vol. II: 253 (dis). H. Aspöck et al., 1980b: 78 (bio).

Considerado como género: H. Aspöck, 1986: 25, 26 (dis, tx). H. Aspöck et al., 1989: 89 (dis). H. Aspöck, 1990: 53 (tx), 54, 56 (dis). Oswald \& Penny, 1991: 65 (1st). H. Aspöck et al., 1991: vol. I: 76, 359 (bio), 111, 115, 116, 117, 360 (tx), 358 (a, gen, mf), 359 (fe, 1, ô, 우), 360, 555, 559 (dis), vol. II: 425 (dis). Nel, 1993: 102, 103 (rf). U. Aspöck \& H. Aspöck, 1994: 39 (tx). H. Aspöck \& Hölzel, 1996: 38 (dis). H. Aspöck, 1998b: 39 (tx). H. Aspöck et al., 2001: 19, 52 (tx), 52 (dis). H. Aspöck, 2002: 32 (1). H. Aspöck \& U. Aspöck, 2007: 99 (dis). Monserrat \& Papenberg, 2010: 63 (rf). Haring et al., 2011: 11-13 (tx), fig.S3 (dis). U. Aspöck et al., 2012: 576, 577, 579, 580 (f), 577 (lst). H. Aspöck \& U. Aspöck, 2013: 12 (tx), 41 (gen).

El actual género Ohmella, exclusivo de la Península Ibérica, fue descrito por H. Aspöck \& U. Aspöck (1968) como un subgénero de Raphidia formado por dos especies citadas en la provincia de Granada. Como especie tipo del subgénero Ohmella se estableció el taxón perteneciente al género Agulla, Agulla voluptaria $\mathrm{H}$. Aspöck \& U. Aspöck, 1968 y la especie Raphidia casta H. Aspöck \& U. Aspöck, 1968. En el año 1971 dichos autores publican una monografía sobre el subgénero Ohmella en donde la especie Raphidia (Ohmella) voluptaria (H. Aspöck \& U. Aspöck, 1968) 
es subdividida en dos subespecies Raphidia (Ohmella) voluptaria voluptaria (H. Aspöck \& U. Aspöck, 1971) y Raphidia (Ohmella) voluptaria lasciva $\mathrm{H}$. Aspöck \& U. Aspöck, 1971 como nueva subespecie. Ambas subespecies son morfológicamente casi idénticas pero con distribuciones diferentes. Al subgénero Ohmella es incorporada la especie Raphidia (Ohmella) libidinosa H. Aspöck \& U. Aspöck, 1971, y posteriormente se describe la especie Raphidia (Ohmella) postulata H. Aspöck \& U. Aspöck, 1977, lo que cierra el actual género Ohmella que se encuentra perfectamente aislado tanto por la morfología de los imagos como de la larvas. Se considera que la especie Ohmella casta queda taxonomicamente aislada respecto a las restantes especies que forman el denominado grupo-baetica.

Dentro de este grupo, la histórica división de la especie en las dos subespecies Ohmella baetica baetica (Rambur, 1842) y Ohmella baetica bolivari (Navás, 1915) es consecuencia del establecimiento del subgénero Ohmella H. Aspöck \& U. Aspöck, 1971. Ambas subespecies son muy similares morfológicamente, pero existen algunas leves diferencias respecto a la genitalia del $\hat{\partial}$ (figs. 66-69), de similar importancia a la que distingue especies próximas de éste $u$ otros géneros. Por el contrario, las diferencias en los segmentos genitales externos y órganos internos de las $ㅇ ㅜ$ son prácticamente inexistentes y se distinguen con mucha dificultad (figs. 111, 112, 135-137). Las áreas de distribución de las dos especies están aparentemente muy bien delimitadas y alejadas una de otra. Desde Monserrat \& Triviño (2013) son consideradas como especies diferentes, y también ahora son consideradas con categoría de especie. Ohmella bolivari solo está citada en el centro occidental de la Península Ibérica (fig. 166) mientras que Ohmella baetica se localiza en el Sistema Penibético (fig. 165). Es muy probable que las citas bibliográficas antiguas de una u otra especie puedan estar mezcladas.

\section{Ohmella baetica (Rambur, 1842)}

Raphidia baetica Rambur, 1842: Rambur, 1842: 437 (a, d, mf), 437, 438 (a, dis), 437 (mf), 438 (dis). Hagen, 1844: 183, 184, 186 (a, dis, rf). Schneider, 1845: 253 (mf), 254 (a, rf), 255 (dis). Hagen, 1850: 369 (rf). Walker, 1853: 210 (a, dis, mf). Costa, 1855: 5 (rf). Rosenhauer, 1856: 867 (dis). Brauer \& Löw, 1857: 53 (lst) - ie. Hagen, 1867: 495 (a), 496 (rf). McLachlan, 1868: 161 (mf, rf), 162 (tx). Girard, 1879: 491-521 (lst). Rostock, 1888 (lst). Albarda, 1891: 76, 79, 81 (rf), 79, 86, 89 (a). McLachlan, 1902: 129 (rf). Navás, 1905a: 37 (a, dis, mf), 38 (dis). Navás, 1908: 35, 36 (c), 35, 45 (a), 36 (dis). Weele, 1910: 88 (dis). Navás, 1910: 46 (rf). Esben-Petersen, 1913a: 6 (dis, lst), 15 (a). Navás, 1915a: 792 (dis). Lauffer, 1921: 49 (bio, dis). Puschnig, 1923 (ie). Navás, 1935: 74 (rf). H. Aspöck \& U. Aspöck, 1968a: 29 (rf). Martín Albaladejo \& Izquierdo Moya, 2006: 134 (dis). Monserrat, 2013: 287 (1st). Monserrat \& Triviño, 2013: 15 (lst).

Raphidilla baetica (Rambur, 1842): Navás, 1919: 65 (a, c), 65, 66 (mf), 66 (dis). Navás, 1923: 71 (c), 72 (dis). Navás, 1928a: 22 (c), 33, 34 (mf), 34 (đ) ) 34, 35 (a), 35 (dis). Navás, 1933a: 121 (1st). Navás, 1934a: 6 (dis). Navás, 1934b: 32 (c), 43 (ð̋, mf), 43, 44 (a, dis). Navás, 1935: 74 (rf). Steinmann, 1964 (a, c). Steinmann, 1967 (rf). Monserrat \& Triviño, 2013: 15 (lst).
Agulla baetica (Rambur, 1842): Bartoš, 1965a: 98 (rf). Bartoš, 1967: 327, 330-333, 336 (ô, dis, mf, rf). Monserrat \& Triviño, 2013: 15 (lst).

Raphidia (Ohmella) baetica Rambur, 1842: H. Aspöck et al., 1980a: 118 (mf, tx), 120 (rf). Díaz-Aranda \& Monserrat, 1988b: 215 (dis).

Raphidia (Ohmella) baetica baetica Rambur, 1842: H. Aspöck et al., 1980a: vol. I: 39, 40 (c), 119 (a, bio, tx), 119, 351 (dis), vol. II: $36($ §), 41 (), 166 (a), 253 (dis). Díaz-Aranda \& Monserrat, 1988b: 215 (dis). Martín Albaladejo \& Izquierdo Moya, 2006: 134 (dis). Monserrat \& Triviño, 2013: 15 (lst).

Ohmella baetica (Rambur, 1842): H. Aspöck et al., 1991: vol. I: 360 (dis, tx), vol. II: 488 (dis). Monserrat, 1996: 16 (lst). H. Aspöck et al., 2001: 52 (tx), 52, 332, 338 (dis). H. Aspöck \& U. Aspöck, 2013: 33 (1st), 46 (gen). Monserrat \& Triviño, 2013: 15, 22, 40 (dis, 1st, tx).

Ohmella baetica baetica (Rambur, 1842): H. Aspöck et al., 1991: vol. I: 89, 361 (bio), 129, 362, 551, 578, 579 (dis), 130 (c), 361

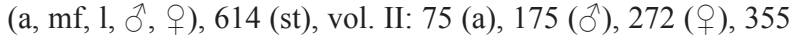
(1), 488 (dis). H. Aspöck \& Hölzel, 1996: 44 (dis). Monserrat, 1996: 16 (lst). H. Aspöck et al., 2001: 52 (tx), 52, 332 (dis). H. Aspöck \& U. Aspöck, 2013: 33 (lst). Monserrat \& Triviño, 2013: 15 (lst).

= Raphidia hispanica Rambur, 1842: Rambur, 1842: 438 (d, mf), 438, 439 (a), 439 (dis). Hagen, 1844: 183 (a, dis, mf). Schneider, 1845: 254, 255 (a, rf), 255 (dis, mf). Hagen, 1850: 369 (rf). Walker, 1853: 211 (dis, mf). Rosenhauer, 1856: 867 (dis). Pictet, 1865: 52, 53, 119 (ie). Hagen, 1866: 287 (dis). Hagen, 1867: 495, 496 (a, mf, rf). Brauer, 1876: 288 (rf). Girard, 1879: 491-521 (1st). Albarda, 1891: 73, 75 (rf), 79 (a) - ie. Weele, 1910: 88 (rf). Navás, 1915b: 861 (rf), 861, 863, 871, 874 (dis), 862 (mf), 862, 863 (a) - ie. Navás, 1924: 130 (dis) - ie. H. Aspöck et al., 1980a: 119 (sin). Díaz-Aranda \& Monserrat, 1988b: 215 (rf). Monserrat \& Triviño, 2013: 15 (lst).

= Raphidilla hispanica (Rambur, 1842): Navás, 1919: 51 (c), 66 (a, dis, mf, ठ̂) - ie. Navás, 1920: 43 (dis) - ie. Navás, 1923: 72 (c, dis). Navás, 1928a: 22 (c), 36, 37 (mf), 37 (a, dis, ठૈ) - ie. Navás, 1933a: 121 (1st) - ie. Navás, 1934a: 6 (dis) - ie. Navás, 1934b: 32 (c), 45 (a, mf, đ̃, ㅇ) - ie. Steinmann, 1964: 216 (a), 217 (c). Monserrat, 1996: 16 (rf). Monserrat \& Triviño, 2013: 15 (lst).

= Agulla voluptaria H. Aspöck \& U. Aspöck, 1968: H. Aspöck \& U. Aspöck, 1968a: 25 (d), 25, 27, 28 (a), 24, 27, 28 (ふ), 25, 28 (). H. Aspöck \& U. Aspöck, 1968b: 62 (tx). H. Aspöck et al., 1980a: 119 (sin). Martín Albaladejo \& Izquierdo Moya, 2006: 134 (1st). Monserrat \& Triviño, 2013: 15 (1st).

= Raphidia (Ohmella) voluptaria $(\mathrm{H}$. Aspöck \& U. Aspöck, 1968): H. Aspöck \& U. Aspöck, 1968b: 62 (a, ô, ᄋ, mf, tx). H. Aspöck \& U. Aspöck, 1971d: 87 (rf), 88 (ठึ). Monserrat \& Triviño, 2013: 15 (lst).

$=$ Raphidia (Ohmella) voluptaria voluptaria $(\mathrm{H}$. Aspöck \& U. Aspöck, 1971): H. Aspöck \& U. Aspöck, 1971c: 26, 27 (ठో, ㅇ), 26, 30, 31 (dis), 31 (c), H. Aspöck \& U. Aspöck, 1977: 185 (dis, tx). Monserrat \& Triviño, 2013: 15 (1st).

TIPO: holotipo de sexo desconocido al faltar el abdomen (Institut Royal des Sciences Naturelles de Belgique, Bruselas).

LOCALIDAD TÍPICA: Sur de España, no se conoce una localidad exacta.

MATERIAL ESTUDIADO: ESPAÑA: Almería: Barranco de Boronol, 30SWG4329, 1.200 m, 28.VI.1983, 1 ईै, V. J. Monserrat (VM), Castala, Sierra Gador, 30SWF0682, 660 m, 11.VII.1985, 2

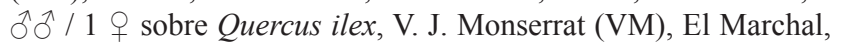
30SWG7432, 440 m, 10.VI.1996, 1 sobre Q. ilex, L. M. DíazAranda (VM), Enix, 30SWF3581, 700 m, 19.VI.1990, 3 우우 
sobre Q. rotundifolia, V. J. Monserrat (VM), Níjar (carretera de), 30SWF7085, $350 \mathrm{~m}, 23 . I V .1988,1$ ô, M. A. Zarazaga (MNCN), Pico Alhamilla, 30SWF6294, 387 m, 30.V.1989, 1 우 sobre Cistus salviifolius, A. Baz (VM), Turrillas, 30SWF6598, 800 m, 30.V.1989, 1 ô sobre Olea europea, A. Baz (VM). Cádiz: Puerto del Boyar, 30STF8770, 1.103 m, 22.VI.2012, 1 \& sobre P. sylvestris, V. J. Monserrat (VM), Sierra del Pinar, 30STF8371, 24.V.1977, 1 đ sobre Abies pinsapo, V. J. Monserrat (VM). Granada: Cañar, 30SVF6287, 1.014 m, 28.VI.2011, 1 ㅇ sobre Q. pyrenaica, V. J. Monserrat (VM), Collado de la Sabina, Sierra Nevada, 30SVG6208, 2.200 m, 10.VII.2012, 1 त / 1 ㅇ sobre Pinus sylvestris, V. J. Monserrat (VM), Deifontes, 30SVG5130, $737 \mathrm{~m}, 1 . \mathrm{V} .1999,1$ ㅇ sobre O. europea, leg.? (EEZ), El Dornajo, 30SVG4741, $1.750 \mathrm{~m}, 22 . V I .2009,1 \hat{\delta} / 1$ ㅇ sobre Berberis vulgaris, V. J. Monserrat (VM), 11.VII.2010, 3 ठ/ 4 ㅇ sobre Q. rotundifolia, V. J. Monserrat (VM), 10.VII.2012, 1 ઈे sobre Q. rotundifolia, V. J. Monserrat (VM), Fuente de Cortichela, 30SVG5904, 1.900 m, 3.VII.1986, sobre $Q$. ilex, 1 ふે, 4 ふふふ/ 1 + sobre P. sylvestris, leg.? (VM), Puerto de Onitar, 30SVG4544, 900 m, 1.VII.1986, 1 sobre O. europea, V. J. Monserrat (VM), Rambla de Aculas, 30SVF7182, 600 m, 2.VI.1986, 1 ㅇ sobre P. halepensis, V. J. Monserrat (VM), Soportujar, 30SVF6387, 940 m, 6.VIII.1972, 1 ㅇ, Vila de Paz (VM), Venta del Chaleco, 30SVF8981, 1.200 m, 2.VI.1986, 1 ․, V. J. Monserrat (VM).

MORFOLOGÍA EXTERNA: Es una especie de tamaño mediano. La cabeza es negra con un dibujo pardo-rojizo. El clípeo y el labro son pardos. El escapo y el pedicelo tienen una coloración amarilla. El flagelo es ocre en su parte basal siendo el resto pardo oscuro. El pronoto es delgado, presentando en su parte anterior un borde ocre y de forma puntual puede aparecer en el tercio anterior una zona clara ocre. En los apéndices locomotores la coxa I es parda con manchas amarillas. Las coxas II y III son de un tono pardo oscuro. El trocánter III puede ser en parte amarillo o pardo en su totalidad. Los demás artejos son amarillentos. En ciertos casos puede existir un oscurecimiento pardo de la cara exterior del fémur II y III. La longitud del ala anterior es de entre 8.8-10.4 $\mathrm{mm}$ en los $\widehat{\partial} \widehat{\partial} \mathrm{y}$ de $9-11 \mathrm{~mm}$ en las 우우 (fig. 33). La venación alar es mayoritariamente de color marrón en la mitad proximal de la costa, subcosta y radio, siendo amarillas las venas del campo costal y la mayoría de las venas de la base alar. En el abdomen los terguitos y esternitos son pardo oscuros con el borde caudal estrecho y amarillo.

SEGMENTOS GENITALES: En los $\widehat{\jmath} \hat{\jmath}$ el proceso del gonocoxito tiene una orientación dorsal y los parámeros se encuentran arqueados en forma de casquete sobre el endofalo (figs. 66, 67, 97). Los estilos son relativamente rectos. Las varillas de la hipovalva son fuertemente divergentes en posición caudal (fig. 67). El hipandrio interno es grande (fig. 88).

En las $9 \circ$ el $7^{\circ}$ esternito es de coloración fuertemente amarilla. El borde cefálico del $7^{\circ}$ terguito no suele alcanzar el espiráculo, existiendo excepciones (ejemplar de El Dornajo en Sierra Nevada) (fig. 111). El ductus receptaculi es muy ancho, reduciendo su diámetro en su región terminal.
BIOLOGÍA: Los imagos se localizan especialmente sobre nanofanerófitos como Cistus, Berberis y Genista, pero también sobre macro o mesofanerófitos como olivares degradados (Olea europea), encinares (Quercus ilex, Q. rotundifolia) o coníferas (Abies, Pinus halepensis, P. sylvestris). Los biotopos son diversos pero xerotermos, por ejemplo maquia abierta de coscoja $(Q$. coccifera). Se localizan en alturas entre los 350-1.900 metros. El número de individuos en las poblaciones suele ser bajo.

FENOLOGÍA: Su fenología es desconocida. H. Aspöck et al. (1991) han conseguido puestas de 9 우 pero a partir de las larvas no se consiguió completar el ciclo. Existen registros entre los meses IV-VIII.

ESTADIOS PREIMAGINALES: Sobre la figura dorsal se diferencian unas manchas aisladas (fig. 151). El dibujo dorsal llega hasta el borde caudal con una clara banda dorsal, que puede estar incompleta en la parte cefálica (fig. 151). Se sospecha que la larva tiene vida edáfica. En este género se ha citado un periodo de desarrollo solo a partir de crías ex ovo, en parte incompletas, no siendo inferior a dos años y siendo lo habitual tres, y la última hibernación transcurre en fase de larva o pupa, por tanto son ciclos vitales de tipo I o II (fig. 18). No se ha demostrado cual de los dos tipos de ciclos vitales es más habitual en su medio natural. De forma experimental se ha comprobado que la fase de pupa, dependiendo del tipo de ciclo vital, se prolonga desde las dos semanas a siete meses. Morfología y aspecto de la larva en figura 151 y en H. Aspöck et al. (1991).

VARIABILIDAD: Existe una gran variación en el tamaño de los $\widehat{\partial} \hat{\delta}$. Las regiones de coloración amarilla en la venación alar son muy variables, pero carecen de valor taxonómico.

DISTRIBUCIÓN: España. Su distribución parece que se limita al Sistema Penibético (fig. 165).

DISCUSIÓN: La morfología que presenta esta especie, coincide ampliamente con las demás especies del género Ohmella. Al margen de su distribución geográfica, la diferenciación con Ohmella bolivari es prácticamente solo posible mediante el estudio de la genitalia en los $\widehat{\partial} \partial^{7}$. Se aprecian diferencias en la forma del proceso del gonocoxito, en la forma de los parámeros y en la curvatura de las varillas de la hipovalva (figs. 66-69). Junto a Ohmella baetica se han citado las especies Ohmella casta en Sierra Nevada, apareciendo esta última a una mayor altitud. Todas las demás especies simpátridas como Hispanoraphidia castellana, Harraphidia laufferi, Subilla aliena y Fibla (F.) hesperica presentan diferencias en la genitalia notables. Muchas de las citas bibliográficas de esta especie podrían tratarse de la especie siguiente, durante mucho tiempo consideradas sinónimas. 
Ohmella bolivari (Navás, 1915)

Raphidia bolivari Navás, 1915: Navás, 1915b: 872 (d, mf), 873 (a), 874 (dis). Martín Albaladejo \& Izquierdo Moya, 2006: 134 (lst). Monserrat \& Triviño, 2013: 15 (lst).

Raphidilla bolivari (Navás, 1915): Navás, 1919: 51 (c). Navás, 1923: 71 (c), 72 (dis). Navás, 1928a: 22 (c), 35 (a), 36 (mf), 36 (dis). Navás, 1933a: 121 (1st). Navás, 1934a: 6 (dis). Navás, 1934b: 32 (c), 44 (mf), 45 (a), 45 (dis). Steinmann, 1964: 216 (a), 217 (c). Monserrat \& Triviño, 2013: 15 (lst).

Raphidia baetica Rambur, 1842: Pictet, 1865: 54, 55 (dis), 117 (mf, ふ̂, ㅇ). Hagen, 1866: 287 (dis). Navás, 1905a: 37 (a), 38 (dis, mf). Navás, 1909a: 378 (dis). Navás, 1915b: 871, 874 (dis). Navás, 1921: 49 (dis).

Raphidia (Ohmella) baetica bolivari Navás, 1915: H. Aspöck et al., 1980a: vol. I: 39, 40 (c), 119 (a, bio, tx), 119, 351 (dis), vol. II: 37 (§) $), 41$ (ㅇ), 253 (dis). Díaz-Aranda \& Monserrat, 1988b: 215 (rf). Monserrat \& Triviño, 2013: 15 (lst).

Ohmella baetica bolivari (Navás, 1915): H. Aspöck et al., 1991: vol. I: 73, 363 (ㅇ), 89, 362 (bio), 129, 362, 551, 578, 579 (dis), 130 (c), 362 (a, fe, mf, 1, ठ̊), 614 (1st) vol. II: 75 (a), 176 (ठ), 272 (), 488 (dis). H. Aspöck \& Hölzel, 1996: 44 (dis). Monserrat, 1996: 16 (lst). H. Aspöck et al., 2001: 53 (tx), 53, 332, 338 (dis). Haring et al., 2011: 5 (dis, lst), 10 (tx), fig.S2 (f). H. Aspöck \& U. Aspöck, 2013: 10 (क), 10, 52 (dis, mf), 33 (lst), 52 (a), 85, 86 (eco). Monserrat \& Triviño, 2013: 15 (lst).

Ohmella bolivari (Navás, 1915): Monserrat \& Triviño, 2013: 15, 22, 41 (dis, lst, tx).

$=$ Raphidia (Ohmella) voluptaria lasciva $\mathrm{H}$. Aspöck \& U. Aspöck, 1971: H. Aspöck \& U. Aspöck, 1971b: 26 (d), 26-28, 30, 32 (dis), 31 (c). H. Aspöck \& U. Aspöck, 1977: 185 (dis, tx). H. Aspöck et al., 1980a: 119 ( $\sin )$. Monserrat \& Triviño, 2013: 15 (lst).

= Raphidia voluptaria lasciva H. Aspöck \& U. Aspöck, 1971 Monserrat \& Triviño, 2013: 15 (lst).

TIPO: Sexo desconocido. Solo existe un fragmento en el Museo Nacional de Ciencias Naturales.

LOCALIDAD TÍPICA: Robledo de Chavela, Madrid (España).

MATERIAL ESTUDIADO: ESPAÑA: Ávila: Ojos Albos, 30TUL7007, 1.226 m, 14.VI.1977, 1 ô sobre Quercus ilex, V. J. Monserrat (VM). Cáceres: Puerto Perales, 29TPE9657, 900 m, 15.V.1980, 1 đ sobre Pinus pinaster, V. J. Monserrat (VM), Villasbuenas de Gata, 29TQE0250, 23.V.1980, 429 m, 1 on, M. Portillo (VM). Madrid: Boadilla del Monte, 30TVK2573, 689 m, 20.VII.2008, 1 ㅇ, D. Papenberg (VM), Ciudad Universitaria, 30TVK3877, 5.VII.1973, $700 \mathrm{~m}$, sobre tronco de plátano, 1 ㅇ, R. Outerelo (VM), Fuentelareyna, 30TVK37, 650 m, 26.V.1934, 1 ô, M. Pujol (MNCN), Las Matas, 30TVK2489, 750 m, 27.VI.1991, 2 우, I. Marcos G. (MNCN), San Lorenzo del Escorial, Arboreto Luis Cevallos, 30TVK0296, $1.030 \mathrm{~m}$, 7.VI.2008, $1 \delta$ en trampa Malaise, 17.VII.2008, errante 1 , V. J. Monserrat (VM). Salamanca: Aldeanueva de Figueroa, 30TTL8858, $920 \mathrm{~m}, 23 . \mathrm{VI} .1977,2$ 우 sobre $P$. pinea, V. J. Monserrat (VM), Espeja, 29TPE9393, 700 m, 29.VI.1980, 2 ठㅊํ / 1 ㅇ sobre Q. ilex, V. J. Monserrat (VM), Garcigalindo, 30TTL5511, $1.060 \mathrm{~m}, 22 . \mathrm{VI} .1977,1$ ㅇ sobre $Q$. ilex, V. J. Monserrat (VM), La Flecha, 30TTL8540, 870 m, 11.VI.1977,

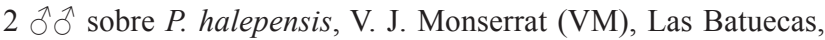
29TQE4283, 21.VII.1982, 1 đ/ 1 1 , C. Urones (VM), Sorihuela, 30TTK7380, $1.020 \mathrm{~m}, 20 . \mathrm{V} .1977,1$ ก sobre Q. pyrenaica, F. Cabezas (VM). Toledo: Navamorcuende, 30TUK4846, $180 \mathrm{~m}$, 22.VII.1978, 1 o sobre P. sylvestris, 1 o sobre Pteridium, V. J. Monserrat (VM). Zamora: Arrabalde, 29TTM6065, 426 m, 4.VII.1993, 1 ㅇ, R. M. Llorden (UNIL), Sazadón, 29TQG4741,
820 m, 10.VII.1977, 1 sobre Q. pyrenaica, V. J. Monserrat (VM). Zaragoza: Fuentesauco, 30TTL8963, 920 m, 23.VI.1977, 1 § sobre Q. ilex, V. J. Monserrat (VM).

MORFOLOGÍA EXTERNA: Su morfología coincide esencialmente con Ohmella baetica. La base de las antenas es parda clara. Los apéndices locomotores son amarillos y los fémures en su cara exterior son pardos.

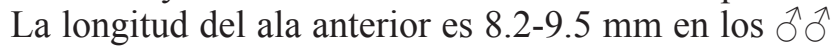
y de 9.4-10.8 $\mathrm{mm}$ en las 우 (fig. 34).

SEGMENTOS GENITALES: Los $\precsim \widehat{\jmath}$ presentan en el proceso del gonocoxito un ápice afilado y su extremo caudal apenas sobresale del mismo (fig. 68). Los parámeros son planos (fig. 68). Las varillas de la hipovalva son divergentes en su extremo, pero sin presentar apenas una curvatura (fig. 69). El hipandrio interno presenta el ala central algo curvada (fig. 89).

Las $q 9$ de Ohmella bolivari son prácticamente idénticas a las de Ohmella baetica pudiendo encontrarse alguna diferencia en el $7^{\circ}$ esternito, que es más alargado (figs. 111, 112, 119). El borde anterior del $7^{\circ}$ terguito alcanza el espiráculo (fig. 112). La bolsa copuladora es idéntica en las dos especies (figs. 135-137).

BIOLOGÍA: Probablemente su biología sea muy similar a la de Ohmella baetica. Hay registros de imagos en bosques mediterráneos aciculifolios sobre macrofanerófitos (Pinus pinaster, P. pinea, P. sylvestris), en laderas orientadas al sur y con escasa vegetación acompañante, o en bosques mediterráneos esclerófilos sobre mesofanerófitos (Quercus ilex, Q. pyrenaica). Su distribución vertical comprende entre los 180-1.226 m. H. Aspöck et al. (1991) indican ocasiones en donde se han producido registros masivos de imagos, pero lo más frecuente son individuos aislados. Se ha observado un caso de fototropismo positivo.

FENOLOGÍA: Existen registros entre los meses de V-VII.

ESTADIOS PREIMAGINALES: La larva es desconocida pero probablemente debe desarrollarse entre la hojarasca.

VARIABILIDAD: Al igual que en Ohmella baetica, la variabilidad es escasa, sobre todo afecta a las regiones de coloración amarilla de los apéndices locomotores y de la venación alar. También existe variación en la longitud del pterostigma pero sin valor taxonómico. Se han identificado algunos ejemplares de $ㅇ$ de esta especie cuyo $8^{\circ}$ terguito no se prolonga en dirección cefálica a la altura del espiráculo, como queda indicado en las claves.

DISTRIBUCIÓN: Centro de España y Portugal. Es posible que su distribución se limite al Sistema Central (fig. 166), pero no se puede descartar la existencia en otros sistemas montañosos. 
DISCUSIÓN: Morfológicamente coincide esencialmente con todas las demás especies pertenecientes al género Ohmella. Ohmella bolivari presenta en los $\widehat{\partial} \hat{o}$ el proceso del gonocoxito más agudo que en Ohmella baetica y su cara ventral apenas sobresale (figs. 66, 68). Otro carácter diferenciador lo representan los parámeros, que en el caso de $O$. bolivari son más planos en su cara dorsal al igual que las varillas de la hipovalva, que en el caso de $O$. bolivari apenas ofrecen una curvatura (figs. 67,69). En las $q$ q una posible diferencia entre las dos especies sería que en el caso de $O$. baetica la zona más esclerotizada del $7^{\circ}$ esternito, en la mayoría de los casos, engloba claramente menos de la mitad del esternito, mientras que en $O$. bolivari ocupa $3 / 4$ partes del mismo (H. Aspöck \& U. Aspöck, 1971c) (figs. 111, 112). Consideramos que se trata de datos puramente estadísticos que no tienen porque ser taxonómicamente vinculantes. En algunos casos la diferenciación es muy complicada. La especie Ohmella bolivari pueden tener en ocasiones una coloración más oscura que Ohmella baetica.

Todas las restantes especies de rafidiópteros de la Península Ibérica se diferencian bien, tanto de Ohmella baetica, como de a O. bolivari, y se han citado como especies simpátridas a $H$. castellana, Atlantoraphidia maculicollis, H. laufferi, S. aliena y Fibla (F.) hesperica.

Ohmella postulata (H. Aspöck \& U. Aspöck, 1977) Raphidia (Ohmella) postulata H. Aspöck \& U. Aspöck, 1977: H. Aspöck \& U. Aspöck, 1977: 186 (d), 188 (a, dis, ô, ㅇ). H. Aspöck et al., 1980a: vol. I: 39 (c), 120 (a, bio, tx), 120, 351 (dis), vol. II: 37 (ð)), 41 (ㅇ), 166 (a), 253 (dis). Monserrat \& Triviño, 2013: 15 (lst).

Ohmella postulata (H. Aspöck \& U. Aspöck, 1977): H. Aspöck et al., 1991: vol. I: 69, 363 (3), 76, 89, 363 (bio), 129, 363, 551, 578, 579 (dis), 130 (c), 363 (a, mf, 1, p), 614 (lst), vol. II: 58, 75 (a), 177 (ठ)), 272 (ㅇ), 343, 355 (1), 489 (dis), 614 (lst). H. Aspöck \& Hölzel, 1996: 44 (dis). H. Aspöck et al., 2001: 53 (tx), 332 (dis). H. Aspöck \& U. Aspöck, 2013: 33 (1st), 138 (gen). Monserrat \& Triviño, 2013: 15, 42 (dis, 1st, tx).

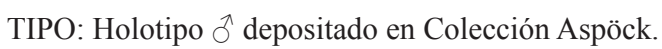

LOCALIDAD TÍPICA: Jijona, Alicante, (España).

MATERIAL ESTUDIADO: ESPAÑA: Alicante: Montesol, 30SYH5078, 800 m, 28.VI.1982, 1 ô/ 2 우 sobre Quercus ilex, V. J. Monserrat (VM), Puerto de Confrides, 30SYH3785, 960 m, 27.VI.1982, 1 ㅎ/ / 2 우 우 sobre Pinus halepensis, 18.VII.1982, 1 त sobre P. halepensis, V. J. Monserrat (VM), Puerto de la Carrasqueta, 30SYH1876, 1.000 m, 27.VI.1982, 4 ठิす / 2 우우

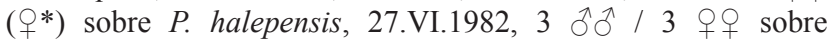
Q. ilex, 18.VII.1982, 1 sobre Q. ilex, V. J. Monserrat (VM). Almería: Rio Claro, 30SWG7973, 1.100 m, 25.V.1983, 1 ઈิ sobre P. halepensis, V. J. Monserrat (VM). Granada: Collado de la Sabina, Sierra Nevada, 30SVG60, 2.200 m, 30.VI.2011, 1 ㅇ sobre P. sylvestris, leg.? (VM), La Sagra, 30SWG49, 958 m, 3 우, Escalera (MNCN). Jaén: Sierra de Cazorla, Nacimiento del Guadalquivir, 30SWG0385, 1.400 m, 1.VII.1978, sobre prado, 1 甲, 2.VII.1978, sobre prado, 1 ㅇ, R. Outerelo (VM). Murcia: Sierra de los Álamos, 30SWH8926, 1.200 m, 10.VI.1982, 1 え sobre $P$. halepensis, R. Outerelo (VM).
MORFOLOGÍA EXTERNA: Esta especie coincide en morfológicamente con Ohmella baetica y $O$. bolivari, siendo algo más pequeña que éstas. La coloración de la base de las antenas y de las patas es, por lo general, algo más clara que en ellas. La longitud del ala anterior es de entre $\widehat{\partial} 7.2-8.5 \mathrm{~mm}$ en los $\partial^{\lambda} \sigma^{\lambda}$ y de 7.6-9.8 mm en las 웅 (fig. 35). En el abdomen los esternitos abdominales en la región caudal están bordeados con una banda amarilla clara.

SEGMENTOS GENITALES: En los $\precsim \widehat{\jmath}$ el proceso del gonocoxito está doblado en forma de pico (fig. 70). El estilo presenta una curvatura más marcada que en otras especies del género (figs. 66, 68, 70, 72, 74). Las varillas de la hipovalva están muy ensanchadas en su base (fig. 71). Los parámeros son discretos y aplanados (fig. 70). El hipandrio presenta el ala central estriada (fig. 90).

En las 99 el extremo caudal del $7^{\circ}$ esternito está mucho más alargado que en las demás especies del género Ohmella, aunque existen excepciones (figs. $113,114)$. Respecto a la genitalia interna, el saco de la bolsa copuladora presenta una fisura esclerotizada muy marcada en posición perpendicular a la estriación longitudinal (fig. 138). El ductus receptaculi mantiene un diámetro relativamente constante en toda su longitud, presentando un grupo de espinas localizadas en su zona media (fig. 138).

BIOLOGÍA: Los imagos se localizan sobre mesofanerófitos en bosques mediterráneos sobre Quercus y Pinus en ambientes xerófilos. Su distribución vertical comprende entre los 800-2.200 m. Las densidades de las poblaciones pueden ser altas según las zonas.

FENOLOGÍA: Existen registros entre los meses de IV-VII (H. Aspöck et al., 1991), y el material estudiado por nosotros comprende los meses V-VII.

ESTADIOS PREIMAGINALES: El dibujo dorsal llega hasta el borde caudal con una clara banda dorsal, que puede estar incompleta en la parte anterior (fig. 152). La figura lateral puede sobrepasarla escasamente, mediante zonas menos pigmentadas de tamaño variable (fig. 152). A partir de crías ex ovo se han registrado periodos de desarrollo de tres años, existiendo un caso puntual de 5 años. El desarrollo transcurrirá con toda probabilidad sobre sustratos edáficos. La última hibernación es en fase de larva y por tanto es un ciclo vital tipo I (fig. 18). El estadio de pupa (en laboratorio) es de 13-14 días. Morfología y aspecto de la larva en figura 152 y en H. Aspöck et al. (1991).

VARIABILIDAD: En las $q 9$ existe una variabilidad en la forma del extremo caudal del $7^{\circ}$ esternito (fig. 120). Se han estudiado una serie de ejemplares de la Sierra de Cazorla (Jaén) y de La Sagra (Granada) en los que el $7^{\circ}$ esternito no presenta esta morfología tan marcada. 
DISTRIBUCIÓN: España. Sudeste de la Península Ibérica (fig. 167). Elemento faunístico extremadamente estacionario, monocéntrico y atlantomediterráneo.

DISCUSIÓN: Ohmella postulata es la especie más cercana a $O$. baetica y $O$. bolivari, pero se diferencia bien en la genitalia en ambos sexos. El resto de los caracteres morfológicos casi coincide plenamente. Junto a Ohmella postulata se ha citado como especies simpátridas a Subilla aliena y Fibla (Fibla) hesperica.

Ohmella libidinosa (H. Aspöck \& U. Aspöck, 1971)

Raphidia (Ohmella) libidinosa H. Aspöck \& U. Aspöck, 1971: H. Aspöck \& U. Aspöck, 1971d: 87 (d, dis), 88 (ð). H. Aspöck \& U. Aspöck, 1971c: 25 (rf), 29 (a), 29, 30 (ㅇ), 29, 31 (c, ơ), 30, 32 (dis), 31 (c). H. Aspöck \& U. Aspöck, 1977: 185 (dis, tx). H. Aspöck et al., 1980a: vol. I: 39 (c), 120 (a, bio, tx), 120, 351 (dis), vol. II: 37 (ठ̂), 41 (†), 166 (a), 253 (dis). Monserrat \& Díaz-Aranda, 1987: 175 (dis). Díaz-Aranda \& Monserrat, 1988a: 219 (dis). Monserrat \& Triviño, 2013: 15 (lst).

Raphidia libidinosa H. Aspöck \& U. Aspöck, 1971: Monserrat \& Triviño, 2013: 15 (1st)

Ohmella libidinosa (H. Aspöck \& U. Aspöck, 1971): H. Aspöck et al., 1991: vol. I: 89, 364 (fe, mf), 129, 364, 551, 578, 579 (dis), 130 (c), 364 (a, bio, ô, o , 1), 614 (lst), vol. II: 75 (a), 52 (mf), $176\left(\delta^{\pi}\right), 272($ ( ) $), 355$ (l), 489 (dis). H. Aspöck \& Hölzel, 1996: 44 (dis). Monserrat, 1996: 12 (lst). H. Aspöck et al., 2001: 53 (tx), 53, 332 (dis). H. Aspöck \& U. Aspöck, 2013: 33 (lst), 133 (gen). Monserrat \& Triviño, 2013: 15, 42 (dis, 1st, tx).

TIPO: holotipo $\widehat{\jmath}$ (colección Aspöck).

LOCALIDAD TÍPICA: Las Torcas, Cuenca (España).

MATERIAL ESTUDIADO: ESPAÑA: Cuenca: Uña, 30TWK8753, 1.146 m, 27.VI.09, 1 क, sobre Pinus sp., V. J. Monserrat (VM). Murcia: Sierra de Espuña, 30SXG2594, 28.VI.1981, 1 ㅇ, M. D. García (VM). Zaragoza: Fuendetodos, 30TXL7070, $750 \mathrm{~m}$, 15.V.2006, 1 \& sobre Quercus ilex, V. J. Monserrat (VM).

MORFOLOGÍA EXTERNA: Es una especie de tamaño mediano. Su morfología coincide en lo general con Ohmella baetica y $O$. bolivari. La longitud del ala anterior es de entre 8.5-9.9 mm en los machos y de 9.8-11.1 mm en las 우우 (fig. 36).

SEGMENTOS GENITALES: En los $\widehat{\jmath}$ el proceso del gonocoxito está orientado en dirección caudal (fig. 72). Las varillas de la hipovalva son muy largas y finas (fig. 73). Los parámeros se encuentran curvados en posición ventrocaudal (figs. 72, 73, 98). El hipandrio interno es de un tamaño muy grande (fig. 91).

En las $ᄋ$ 우 el intersegmento $\mathrm{S} 7 / 8$ presenta unas plaquitas eclerotizadas características (figs. 114, 115, 121). Entre el atrio y el sáculo de la bolsa copuladora no existe ningún tipo de diferenciación, dando una apariencia de unidad (fig. 139). El ductus receptaculi es estrecho y mantiene su diámetro en toda su longitud, y las pequeñas espinas se agrupan en bandas longitudinales (fig. 139).

BIOLOGÍA: Se tienen pocos datos respecto a su biología al existir muy pocos registros. Los imagos han sido capturados en ecosistemas templados, sobre vegetación baja, en pinares con una estructura arbórea clareada con un rico sotobosque. En algunos biotopos aparece también sobre Quercus. La distribución vertical comprende entre los 750 y 1.400 m (H. Aspöck et al., 1991), y el material estudiado por nosotros comprende los 750-1.146 m. Las densidades de población son generalmente bajas, existiendo algún caso de captura masiva (H. Aspöck \& U. Aspöck, 1971c).

FENOLOGÍA: Existen registros entre los meses de V-VI.

ESTADIOS PREIMAGINALES: Las larvas presentan en la banda dorsal una bifurcación en el extremo cefálico (fig. 153). Se han registrado periodos de desarrollo de al menos dos años. El desarrollo sobre sustrato edáfico no está confirmado, aunque se considera muy probable (H. Aspöck et al., 1991). Morfología y aspecto de la larva en figura 153 y en H. Aspöck et al. (1991).

VARIABILIDAD: La variabilidad muy escasa y sin valor taxonómico.

DISTRIBUCIÓN: Este de la Península Ibérica (fig. 168). Elemento faunístico extremadamente estacionario, monocéntrico y atlantomediterráneo.

DISCUSIÓN: Aunque casi coincide morfológicamente con las restantes especies del género Ohmella, se diferencia claramente en su genitalia. En los $\widehat{\partial} \hat{\partial}$ la hipovalva es más estrecha que en las demás especies (figs. 67, 69, 71, 73, 75). En las $ᄋ+$ aparecen las placas esclerotizadas en el intersegmento $S 7 / 8$ exclusivas de esta especie (figs. 114, 115), y no existe una separación entre el atrio y la bolsa copuladora. El $d u c-$ tus receptaculi es aparentemente más estrecho y sus espinas están más marcadas que en las demás especies del género Ohmella (figs. 135, 138, 139).

Especies simpátridas de Ohmella libidinosa son Atlantoraphidia maculicollis, Hispanoraphidia castellana, Harraphidia laufferi, Subilla aliena y Fibla (Fibla) hesperica.

Ohmella casta (H. Aspöck \& U. Aspöck, 1968)

Agulla casta H. Aspöck \& U. Aspöck, 1968: H. Aspöck \& U. Aspöck, 1968a: 26 (d), 26, 28 (đ), 26, 29 (ㅇ), 28, 29 (a). Monserrat \& Triviño, 2013: 15 (1st).

Raphidia casta (H. Aspöck \& U. Aspöck, 1968): Monserrat \& Triviño, 2013: 15 (lst).

Raphidia (Ohmella) casta (H. Aspöck \& U. Aspöck, 1968): H. Aspöck \& U. Aspöck, 1968b: 62 (a, mf, tx). H. Aspöck \& U. Aspöck, 1971c: 30 (ㅇ), 30, 31 (ふ), 30, 32 (dis), 31 (c). H. Aspöck \& U. Aspöck, 1971d: $80($ đ $)$. H. Aspöck \& U. Aspöck, 1977: 185 (dis, tx). H. Aspöck et al., 1980a: vol. I: 39 (c), 120 (bio, 1, mf, tx), 121, 351 (dis), vol. II: 37 (ð) ), 41 (), 166 (a), 253 (dis). Monserrat \& Triviño, 2013: 15 (1st).

Ohmella casta (H. Aspöck \& U. Aspöck, 1968): H. Aspöck et al., 1991: vol. I: 68, 365 (ठ) , 89, 365 (bio), 129, 365, 551, 578, 579 (dis), 130 (c), 365 (a, fe, 1, p, ㅇ), 614 (lst), vol. II: 34, 58, 75 (a), 46, 52 (mf), 177 (ふ), 272 (), 355 (l), 489 (dis). 
H. Aspöck \& Hölzel, 1996: 44 (dis). H. Aspöck et al., 1999: 74 (dis). H. Aspöck et al., 2001: 53 (tx), 53, 332 (dis). H. Aspöck \& U. Aspöck, 2013: 33 (lst), 48 (gen). Monserrat, 2013: 287 (bio, dis, 1st). Monserrat \& Triviño, 2013: 15, 41 (dis, 1st, tx).

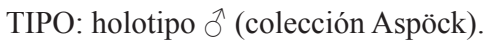

LOCALIDAD TÍPICA: Carretera del Veleta, Sierra Nevada, Granada (España).

MATERIAL ESTUDIADO: ESPAÑA: Granada: Fuente de Cortichuela, 30SVG5904, $1.900 \mathrm{~m}$, 3.VII.1986, 1 ô sobre Pinus sylvestris, leg.? (VM). Jaén: Sierra de Cazorla, Arroyo de Linarejos, 30SWG0797, 22.VI.1984, 2 ठิે, J. Alba (VM), Roblehondo, 30SWG0899, 1.300 m, 9.VI.1991, 1 ô, J. L. Yela (VM).

MORFOLOGÍA EXTERNA: Especie de tamaño pequeño hasta mediano, y de aspecto delgado. Su morfología externa coincide en lo general con Ohmella baetica. La longitud del ala anterior es de entre 7-8.2 mm en los $\widehat{\partial} \widehat{\partial}$ y 7.8-9.5 mm en las 우우 (figs. 37, 40, 41).

SEGMENTOS GENITALES: En los $\widehat{\jmath} \widehat{\jmath}$ el gonocoxito está suavemente abombado con un proceso romo poco desarrollado y escasamente delimitado (fig. 74). La hipovalva y sus varillas divergentes son cortas (fig. 75). Los parámeros son discretos y quedan situados entre los gonocoxitos. El endofalo presenta en su extremo caudal una invaginación característica (fig. 75).

En las $O+9$, en el $8^{\circ}$ terguito, la cara cefálica no está desarrollada en dirección anterior (fig. 116, 122). No se disponen de datos sobre la genitalia interna.

BIOLOGÍA: El desarrollo transcurre en el sustrato edáfico. Los adultos han sido localizados en alturas entre los 1.300-1.900 m en biotopos con vegetación exclusivamente arbustiva (sobre todo Berberis) o sobre macrofanerófitos (Pinus sylvestris). Las densidades de población parecen ser elevadas.

FENOLOGÍA: Existen registros entre los meses de VI-VII.

VARIABILIDAD: La variabilidad muy escasa y sin valor taxonómico.

ESTADIOS PREIMAGINALES: Se diferencian bien de las demás especies de Ohmella por las bandas laterales delgadas interrumpidas por puentes pigmentarios (figs. 141-143, 154). A partir de crías ex ovo se han registrado periodos de desarrollo de tres años y ocasionalmente dos años. Se han hallado larvas en el detritus de las raíces de Berberis (H. Aspöck et al., 1991). La última hibernación es en estadio de larva o pupa, y por tanto son ciclos de tipo I y II (fig. 18). En algunas larvas se pasa a la fase de pupa en agosto, antes de la hibernación, mientras otros individuos en primavera (abril). Por tanto la fase de pupa puede de prolongarse desde pocos días hasta algunos meses. Morfología y aspecto de la larva en figuras 141-143, 154 y en H. Aspöck et al. (1991).
DISTRIBUCIÓN: Sudeste de la Península Ibérica (fig. 169). Elemento faunístico extremadamente estacionario, monocéntrico y probablemente atlantomediterráneo. Es posible que su distribución se limite a los Sistemas Béticos y Penibéticos.

DISCUSIÓN: Ohmella casta es una especie taxonómicamente diferenciada tanto en el $\widehat{\partial}$ como en la . Representa el taxón hermano del resto de las especies conocidas del género Ohmella. La morfología externa de $O$. casta es muy parecida a la de $O$. baetica y $O$. bolivari. Ohmella casta es algo más pequeña, su venación alar presenta menos regiones amarillas en la base del ala. Respecto a los $\widehat{\partial} \widehat{\partial}$, en Ohmella casta el proceso del gonocoxito está menos definido que en las otras dos especies (figs. 74, 75) y los parámeros quedan completamente englobados por los gonocoxitos (fig. 74), al contrario que en las restantes especies del género Ohmella, en donde los parámeros se sitúan fuera de los gonocoxitos (figs. 66, 68, 70, 72). Respecto a las $9+9$ las diferencias son menos claras entre $O$. casta y las restantes especies del género Ohmella. Mientras que en las demás especies la cara anterior del $8^{\circ}$ terguito se prolonga en dirección anterior (figs. 111-114, 119-121), en O. casta el $8^{\circ}$ terguito carece de esta prolongación (figs. 116, 122).

Ohmella baetica y Ohmella casta coinciden en áreas de distribución, aunque esta última suele aparecer en zonas más altas, y no se descarta que haya biotopos de Sierra Nevada en donde aparezcan ambas especies. Las especies Harraphidia laufferi y Subilla aliena también han sido citadas de Sierra Nevada, pero por debajo de los $1.500 \mathrm{~m}$.

Género Venustoraphidia H. Aspöck \& U. Aspöck, 1968

Venustoraphidia H. Aspöck \& U. Aspöck, 1968b: 60 (d), [especie tipo designada por Aspöck \& Aspöck, 1973: Raphidia nigricollis Albarda, 1891].

Considerado como subgénero de Raphidia Linnaeus, 1758: H. Aspöck \& U. Aspöck, 1974d: 166, 167 (rf). H. Aspöck et al., 1974b: (mon), 101, 109 (gen), 108-109 (a, mf), 109 (bio, 1, §̂, ㅇ). H. Aspöck et al., 1975: 373 (1). H. Aspöck, 1979: 249 (dis). H. Aspöck et al., 1980a, vol. I: 86 (lst), 87 (dis), 112 (rf), 125 (tx), vol. II: 259 (dis).

Considerado como género: H. Aspöck et al., 1983b: 27 (rf), 28 (tx). H. Aspöck, 1986: 25 (dis), 26 (tx). H. Aspöck et al., 1989: 90, 106 (dis), 108 (tx). Aspöck, 1990: 55 (dis), 56, 58 (tx). H. Aspöck et al., 1991, vol. I: 76, 77, 372 (1), 112, 116, 371, 372 (tx), 371 (mf, ふै), 372 (bio, fe, + ), 372, 559 (dis), vol. II: 492 (dis). Oswald \& Penny, 1991: 66 (lst). H. Aspöck \& Hölzel, 1996: 38 (dis). H. Aspöck et al., 2001: 54 (dis, tx). H. Aspöck, 2002: 38 (1), 44, 46 (pa). H. Aspöck \& U. Aspöck, 2007: 100 (tx), 102 (dis). Letardi \& Pantaleoni, 2007: 89 (lst). Haring et al., 2011: 9 (tx), fig. S5 (dis). Haring \& H. Aspöck, 2012: 577 (lst), 579 (f). Monserrat \& Papenberg, 2012: 292 (rf), 293 (mf), 293, 294 (a), 293-295 (ð̋), 294 (bio, ㅇ), 296 (fe, 1, p, tx), 296 (tx), 296, 300 (dis). H. Aspöck \& U. Aspöck, 2013: 12 (tx), 43 (gen).

En el año 1968 H. Aspöck \& U. Aspöck crearon, dentro del género Raphidia, el subgénero Venustoraphidia, 
con una única especie Raphidia nigricollis (Albarda, 1891). Esta especie se encontraba morfológica y taxonómicamente aislada de todas las demás especies conocidas, y está citada del centro y sudeste de Europa. A esta especie se le uniría posteriormente Raphidia (Venustoraphidia) renate H. Aspöck \& U. Aspöck, 1974, cuya distribución queda delimitada a la Península del Peloponeso (Grecia) (H. Aspöck et al., 1974b; H. Aspöck et al., 1980a; H. Aspöck et al., 2001). El hallazgo de una tercera especie: Venustoraphidia conviventibus Monserrat \& Papenberg, 2012 en el norte de la Península Ibérica (fig. 170), ha ampliado la distribución conocida del género hacia el suroeste de Europa.

\section{Venustoraphidia conviventibus Monserrat \& Papenberg, 2012 \\ Venustoraphidia conviventibus Monserrat \& Papenberg, 2012: Monserrat \& Papenberg, 2012: 297 (a, d), 297-300 (ठ̊), 298 (bio, fe, mf), 298, 299 (dis), 300 (c). H. Aspöck \& U. Aspöck, 2013: 33 (1st), 77 (gen). Monserrat \& Triviño, 2013: 15, 44 (dis, lst, tx).}

TIPO: holotipo $\widehat{\jmath}$ (colección V. J. Monserrat).

LOCALIDAD TÍPICA: Ataun, Aia, Parque Natural de Aralar, Urkillaga, Guipúzcoa (España).

MATERIAL ESTUDIADO: ESPAÑA: Guipúzcoa: Ataun, Aia, Parque Natural de Aralar (Urkillaga), 30TWN6858, 614 m, 1 ठ recolectado mediante trampa aérea sobre Quercus ilex ilex instalada desde el 27-VI al 8-VII-2011, S. Pagola Carte leg. (Colección V. J. Monserrat).

MORFOLOGÍA EXTERNA: Especie de tamaño pequeño. La longitud del ala anterior es de $7 \mathrm{~mm}$ en el $\widehat{\partial} \hat{o}$ (fig. 38). En la cabeza el clípeo y el labro son pardos. El escapo es negro, el pedicelo es ocre con una banda parda central que lo rodea en forma de anillo. El flagelo en su tercio proximal es ocre y en los dos tercios distales negro. El pronoto es totalmente negro. La coxa I es parda con la zona distal ocre, las coxas II y III son totalmente pardo oscuras. El fémur III es pardo. Los demás artejos son amarillos, excepto los tarsos II, III y IV que son pardos. La venación alar es casi en su totalidad parda, excepto la región proximal de la Costa y el Radio que es amarilla. El pterostigma es pardo. La región distal ocupada por Radio y su sector presenta una vena bifurcada y otra no en todas las alas (fig. 38).

SEGMENTOS GENITALES: El $\partial^{\lambda}$ presenta el $8^{\circ}$ terguito bien diferenciado del $8^{\circ}$ esternito, sin formar un anillo continuo (fig. 76). El $9^{\circ}$ terguito se alarga dorsalmente con su borde anterior convexo. El $9^{\circ}$ esternito presenta escasa pigmentación en su región central (fig. 77) y está bien individualizado del gonocoxito por una patente sutura (figs. 76, 77). El gonocoxito es proporcionalmente pequeño y porta un pequeño apodema del estilo que divide al gonocoxito en una región dorsal más pequeña y una ventral que presenta un borde ventro-caudal redondeado (fig. 76). Los estilos, dentro del modelo existente en el género Venustoraphidia, son grandes, falciformes y suavemente recurvados hacia arriba y levemente hacia afuera (fig. 76). La hipovalva impar está uniforme y progresivamente adelgazada desde su base hacia su margen caudal (fig. 77) y presenta dos costillas centrales muy esclerificadas con un recorrido muy próximo y paralelo, convergiendo en su ápice caudal (fig. 77) y posee unos suaves engrosamientos en la parte basal bifurcada (fig. 77), siendo sus extremos anteriores muy patentes, divergentes y recurvados hacia afuera (fig. 77). El esclerito impar sobre la hipovalva es discreto y presenta dentículos orientados en dirección anterior (figs. 76, 99). La $q$ es, por el momento, desconocida.

BIOLOGÍA: Su biología es desconocida, pero debe ser acorde con lo citado para las otras especies del género conocidas. El ejemplar sobre el que se basó la descripción de esta especie fue capturado a $614 \mathrm{~m}$ de altitud, en trampa colgada sobre Quercus ilex ilex (Monserrat \& Papenberg, 2012), junto a un ejemplar + de Subilla aliena. Probablemente en las especies de este género hasta ahora conocidas exista una preferencia por colonizar Quercus sp. (la especie ibérica ha sido hasta ahora recolectada sobre Quercus ilex ilex), pero se desarrollan en otras muchas especies arbóreas, mostrando preferencia por biocenosis antrópicas donde se cultiven árboles frutales, sobre todo Pyrus y Malus. Las especies $V$. nigricollis y $V$. renate parecen ser xerotermas. Se han citado en Europa Central en cotas inferiores a $500 \mathrm{~m}$, alcanzando en el sur de Europa los 1.100 m, y en España a 614 m. En las especies no ibéricas las poblaciones suelen ser por lo general pequeñas (H. Aspöck \& U. Aspöck, 1974d; H. Aspöck et al., 1974b, 1991). Esto debe coincidir con el caso de la especie ibérica, ya que estas trampas, donde se halló el único ejemplar conocido, se mantuvieron instaladas al año siguiente de forma continuada (en la misma localidad, incluso una de las trampas en el mismo árbol) entre los periodos desde finales de $\mathrm{V}$ a finales de VII, y se efectuaron muestreos intensivos sobre la vegetación de la localidad tipo en V y VI de ese año, sin obtener ningún otro resultado (Monserrat \& Papenberg, 2012).

FENOLOGÍA: Su fenología se desconoce. Como hemos indicado, el ejemplar conocido ha sido capturado en una trampa que estuvo instalada entre finales del mes VI y principos del VII. Las otras especies del género presentan ciclos monovoltinos, y la actividad de sus imagos transcurre durante los meses de V-VII.

\section{VARIABILIDAD: Desconocida.}

ESTADIOS PREIMAGINALES: Los estados preimaginales, tanto la larva como la pupa son desconocidos. De este género se conocen las larvas de las especies $V$. nigricollis y $V$. renate. (Aspöck et al., 1974a, 1991). Su periodo de desarrollo se prolonga dos años pudiendo ser ocasionalmente tres años. 
La última hibernación transcurre en fase de larva y por tanto es un ciclo vital de tipo I (fig. 18). La fase de pupa se desarrolla entre 10-20 días. En los segmentos abdominales medios, la figura dorsal solo es visible en la zona central del segmento. La pigmentación es más débil en la región caudal del segmento o puede estar incluso interrumpida. No existe una banda dorsal continua. Las bandas laterales son estrechas y se pueden encontrar reducidas o interrumpidas mediante la aparición de manchas pálidas. Las manchas laterales presentan grandes zonas claras que se extienden hacia el extremo caudal y de forma escasa tras la sutura dorsal. La figura ventral se delimita a la zona central, presentando una forma rectangular, no existiendo una banda ventral. La figura dorsal del $8^{\circ}$ segmento abdominal presenta un borde caudal llamativamente rectilíneo. Las larvas en ambas especies se diferencian con dificultad, y es de destacar su sorprendentemente pequeño tamaño (H. Aspöck et al., 1974a, 1974b, 1991). La larva de V. conviventibus es, por el momento, desconocida (Monserrat \& Papenberg, 2012), pero debe parecerse a lo indicado para las otras especies. En otras especies del género, el desarrollo juvenil transcurre exclusivamente debajo de las cortezas de los árboles.

DISTRIBUCIÓN: Probablemente se trate de un elemento faunístico estacionario, monocéntrico ibéricoseptentrional, quizás atlantomediterráneo, de marcado carácter preglaciar relicto.

DISCUSIÓN: Es una especie taxonómicamente diferenciada en el $\hat{\sigma}$ (se desconoce la $q$ ). Representa el taxón hermano del resto de las dos especies conocidas del género Venustoraphidia. Respecto a su morfología, resulta llamativo en los segmentos pregenitales que el $8^{\circ}$ terguito y el $8^{\circ}$ esternito sean independientes (fig. 76) y no se encuentran fusionados formando un anillo, hecho que ocurre en las otras dos especies conocidas de este género. A su vez, llama la atención que el $9^{\circ}$ terguito y el $9^{\circ}$ esternito no estén más o menos fusionados al gonocoxito, sino bien delimitados y marcadamente individualizados de él por una clara sutura (fig. 76) entre otras diferencias, lo que demuestra el aislamiento de este elemento con respecto a las otras dos especies del género (Monserrat \& Papenberg, 2012). A su vez la especie Venustoraphidia conviventibus se encuentra marcadamente aislada taxonómicamente de las restantes especies citadas en la Península Ibérica.

Familia Inocelliidae Navás, 1913

Inocellia Schneider, 1843: Schneider, 1843: $84-91$ (d, 1, mf, mon, tx). Hagen, 1844: 181 (rf). Brauer, 1852: 72-75 (tx). Costa, 1855: 2 (rf), 6, 7 (mf). Brauer \& Löw, 1857: 54 (lst). Brauer, 1876: 288 (lst). Girard, 1879: 491-521 (rf). Redtenbacher, 1886 (mf). Albarda, 1891: 76, 82 (rf). Sharp, 1895 (1st). EsbenPetersen, 1913a: 11 (mf). Navás, 1915h (mf). Navás, 1918c: 92 (tx). Comstock, 1918: 172 (mf). Stitz, 1927a: 4 (tx).
Inocelliini Navás, 1913: Navás, 1913b: 11 (d).

Inocelliini Navás sensu Navás, 1918c partim: Navás, 1918c: 91 (mf), 92 (tx). Lestage, 1928: 30 (rf). Navás, 1928a: 46 (tx). Navás, 1934b: 56 (a, c).

Inocelliidae Navás, 1913: Navás, 1916: 509 (d, a). Navás, 1919: 70 (c). Lestage, 1928: 32 (c). Navás, 1928a: 44 (c). Navás, 1934b: 11, 49, 50 (c). Carpenter, 1936: 93 (mf). Brues et al., 1954: 205 (mf, tx). Tjeder, 1954: 26 (mf). Metzger, 1958 (bio, mf). Acker, 1960: 35-47, 54 (mf). Martynova, 1961: 71-83 (tx). Martynova, 1962: 270-272 (mf). Meinander, 1962: 15 (c), 18 (mf). Steinmann, 1963: 184 (tx). H. Aspöck \& U. Aspöck, 1964: 142 (mf). H. Aspöck \& U. Aspöck, 1966a: 105, 107 (dis), 106 (tx), 129, 130 (ð). Steinmann, 1967 (mf). H. Aspöck \& U. Aspöck, 1968b: 53, 63 (tx). Rohdendorf, 1969 (paleo). Tjeder, 1970 (mf). Tuxen, 1970 (mf). H. Aspöck \& U. Aspöck, 1971a: 5, 6 (tx), 7 (c). Lauterbach, 1972 (mf, tx). H. Aspöck et al., 1974a: 45 (rf), 51 (1). H. Aspöck et al., 1974c: 22 (bio). U. Aspöck, 1974 (dis, tx). U. Aspöck, 1975: 540 (dis). Lauterbach, 1974: 1 (f). Achtelig, 1975b: 228 (mf). H. Aspöck et al., 1975: 373 (eco). Matsuda, 1976 (mf). Achtelig, 1977 (mf, +). Mamaev et al., 1977 (bio, eco, 1, mf). H. Aspöck et al., 1980a: 4 (st). Hennig, 1981 (f). Henry, 1982: 470-482 (mf). Gepp, 1984: 189 (c, 1), 205, 206 (gen). Dorokhova, 1987: 28, 29 (dis, mf, đ). H. Aspöck \& U. Aspöck, 1985: 45 (dis). H. Aspöck et al., 1985b: 38 (tx). H. Aspöck, 1986: 21, 24 (dis) 22 (tx). H. Aspöck, 1987: 149, 155, 157 (dis). Greve, 1987: 35 (rf). Tauber, 1987: 124 (c, gen). H. Aspöck et al., 1989: 67 (gen), 73, 85 (dis). H. Aspöck, 1990: 51 (tx), 55 (dis). H. Aspöck, 1991: 27 (tx). H. Aspöck \& U. Aspöck, 1991: 522 (mf). H. Aspöck et al., 1991: vol. I: 124, 172-179 (c), 478 (a, mf, 1, ô, +), 479 (bio, dis, tx), vol. II: 522, 523 (dis). U. Aspöck, 1991: 242 (dis). Czechowska \& Dobosz, 1991: 139 (1st). Oswald \& Penny, 1991: 63 (cat). U. Aspöck et al., 1992: 181 (1), 181, 182 (dis). U. Aspöck et al., 1994a: 393-395, 398-401 (bio), 401 (すへ). Günther, 1994: 221-225 (rf). Ren \& Hong, 1994: 103-108 (f). Willmann, 1994: 191 (tx). H. Aspöck \& Hölzel, 1996: 33, 44, 45 (dis), 44 (tx). U. Aspöck \& H. Aspöck, 1996: 283, 284 (dis). Penny et al., 1997: 89 (cat). Wachmann \& Saure, 1997 (mf). U. Aspöck \& H. Aspöck, 1999a: 4 (dis), 5, 7 (bio), 6 (mf). H. Aspöck et al., 2001: 16 (1st), 56 (dis, tx). Vas et al., 2001: 50 (t). H. Aspöck, 2002: 39 (l), 40, 43 (bio). H. Aspöck \& U. Aspöck, 2007: 100 (rf). Monserrat \& Papenberg, 2006: 211 (rf). Haring et al., 2011: 1 (gen), 2 (dis), 9 (tx). H. Aspöck \& U. Aspöck, 2012: 217 (gen), 218 (bio, h, p), 218, 219 (dis), 219 (tx). H. Aspöck et al., 2012: 566 (rf). Monserrat \& Triviño, 2013: 15, 22 (cat, dis, gen, 1st, sin, tx).

Inocelliinae Navás, 1913: Handlirsch \& Beier, 1936: 1402-1413 (gen, mf). Berland \& Grassé, 1951: 18-22 (gen). Friedrich, 1953 (tx). Hoffmann, 1962: 258 (rf).

Inocelliinae Navás sensu Inocelliini Navás, 1918c partim: Lestage, 1928: 30 (tx). Carpenter, 1936: 93 (tx).

Navás (1913b) subdividió la familia Raphidiidae al crear las tribus Raphidini e Inocellini, basándose en la existencia o no de una vena que divide al pterostigma. Posteriormente elevó sus tribus a nivel de familia describiendo numerosos de géneros en cada una de ellas. Desde entonces la identidad de la familia Inocelliidae, basada en multitud de caracteres diferenciales, se ha mantenido frente a la familia Raphidiidae.

Actualmente la Familia Inocelliidae Navás, 1913 engloba 7 géneros: Inocellia Schneider, 1843, Fibla Navás, 1915, Negha Navás, 1916, Parainocellia 
H. Aspöck \& U. Aspöck, 1986, Indianoinocellia U. Aspöck \& H. Aspöck, 1970, Amurinocellia H. Aspöck \& U. Aspöck, 1973 y Sininocellia Yang, 1985. En la Península Ibérica sólo está citado el género Fibla con la especie Fibla (Fibla) hesperica Navás, 1915. Su distribución global es similar a la de la familia Raphidiidae.

\section{Género Fibla Navás, 1915}

Fibla Navás 1915d: 25 (d, a, mf), [especie tipo por designación original Fibla hesperica Navás, 1915].

Navás, 1918c: 91, 82 (tx). Navás, 1919: 6 (1st), 70 (c). Lestage, 1928: 32 (c). Steinmann, 1963: 184 (lst). H. Aspöck \& U. Aspöck, 1966b: 106 (a, rf), 106, 126 (ㅇ), 127 (ô), 129 (tx). H. Aspöck \& U. Aspöck, 1968b: 53 (a), 53, 63 (tx). H. Aspöck \& U. Aspöck, 1971a: 5 (rf, tx), 6 (a). Lauterbach, 1972: 219 (dis, rf), 231-237, 242 (mf), 247 (a), 249 (tx). H. Aspöck, 1986: 26 (dis, tx). H. Aspöck et al., 1989: 94, 106 (dis). Oswald \& Penny, 1991: 63 (lst). H. Aspöck et al., 1991: vol. I: 76, 480 (bio), 106, 107-119, 479, 480 (tx), 479 (mf, ô, o ), 480 (1, pa), 480, 559, 569 (dis), 555 (rf), vol. II: 524 (dis). Nel, 1993: 106 (rf). U. Aspöck \& H. Aspöck, 1994: 36-39, 41 (dis), 36, 41 (tx). H. Aspöck \& Hölzel, 1996: 45 (dis). U. Aspöck \& H. Aspöck, 1999b: 490 (tx). H. Aspöck et al., 2001: 56 (dis, tx). H. Aspöck, 2002: 39, 44 (l), 44 (pa). H. Aspöck \& U. Aspöck, 2007: 100 (rf). Liu et al., 2010: 232 (ð). Monserrat \& Papenberg, 2010: 63 (rf). H. Aspöck et al., 2012: 566 (rf), 568 (tx). Liu et al., 2012: 233, 239 (a), 234 (rf), 239 ()), 240 (f). H. Aspöck \& U. Aspöck, 2013: 12 (tx), 144 (gen).

Fiblini Navás, 1918 partim: Navás, $1918 \mathrm{c}: 91$ (d). Lestage, 1928: 30 (rf). Navás, 1928a: 44, 45 (mf). Navás, 1934b: 50 (mf). Carpenter, 1936: 93 (tx).

Fiblinae Navás sensu Fiblini Navás, 1918c partim: Lestage, 1928: 30 (tx). Carpenter, 1936: 93 (tx).

Fibla es un género bien delimitado por su morfología y su genitalia. Engloba en la actualidad cuatro especies agrupadas en dos subgéneros: El subgénero Fibla s.str. Navás, 1915, con las especies Fibla (Fibla) hesperica Navás, 1915, Fibla (Fibla) maclachlani (Albarda, 1891) y Fibla (Fibla) peyerimhoffi (Navás, 1919) y el subgénero Reisserella H. Aspöck \& U. Aspöck, 1971, con la especie Fibla (Reisserella) pasiphae (H. Aspöck \& U. Aspöck, 1971). Su distribución comprende la Península Ibérica, Sicilia, Córcega, Cerdeña, Creta, Marruecos, Argelia y Túnez.

Subgénero Fibla Navás, 1915

Fibla Navás, 1915d: 25 (d, a, mf), [especie tipo por designación original: Fibla hesperica Navás, 1915]. Navás, 1918c: 92 (tx).

Considerado como subgénero de Inocellia Schneider, 1843: H. Aspöck \& U. Aspöck, 1971b: 271 (rf). H. Aspöck et al., 1975: 373 (1). H. Aspöck et al., 1980a: vol. I: 128 (a), 128, 131 (tx), 129 (dis), vol. II: 260, 262 (dis).

Considerado como subgénero de Fibla Navás, 1915: H. Aspöck et al., 1989: 94, 107 (dis). H. Aspöck 1990: 51 (1st), 55, 56 (dis), 56 (tx). H. Aspöck et al., 1991: vol. I: 479-481 (ठో), 480 (mf), 480, 481 (a, + , tx), 481 (bio, pa), 481, 559 (dis), vol. II: 524 (dis). H. Aspöck \& Hölzel, 1996: 45 (dis). H. Aspöck et al., 2001: 56 (dis, tx). H. Aspöck, 2002: 39 (1). Letardi \& Pantaleoni, 2007: 89 (lst).

= Burcha Navás 1915d: 27 (d, a), [especie tipo por designación original: Inocellia maclachlani Albarda 1891]: Navás, 1918c: 92 (tx). Navás, 1919: 6 (dis), 70, 72 (c). Navás, 1925: 48 (lst).
Lestage, 1928: 32 (sin). Navás, 1928a: 32 (tx). Osorio Rebellón, 1929: 35 (rf). Navás, 1933a: 121 (lst). Navás, 1934a: 7 (lst). Navás, 1934b: 56 (a, c). Carpenter, 1936: 93 (tx). Steinmann, 1963: 184 (lst). H. Aspöck \& U. Aspöck, 1966b: 106 (rf). Steinmann, 1967 (tx). H. Aspöck \& U. Aspöck, 1971b: 271 (sin). H. Aspöck et al., 1980a: 131 (sin). Oswald \& Penny, 1991: 63 (1st).

Considerado como subgénero de Inocellia Schneider, 1843: H. Aspöck \& U. Aspöck, 1968b: 63 (tx).

= Estoca Navás, 1918c: 91, 92 (d), [especie tipo por monotipia: Estoca peyerimhoffi Navás, 1919]: Lestage, 1928: 32 (sin). Navás, 1934b: 51 (a, mf), 52 (c). Carpenter, 1936: 93 (tx), 95 (sin). Steinmann, 1963: 184 (1st). Aspöck, 1966b: 106 (rf).

Fibla s.str. es un subgénero muy bien delimitado y muy homogéneo formado por tres especies muy emparentadas: Fibla (Fibla) hesperica Navás, Fibla (Fibla) maclachlani y como taxón hermano sería Fibla (Fibla) peyerimhoffi. Se conoce de la Península Ibérica, Sicilia, Córcega y Cerdeña, Marruecos, Argelia y Túnez. Las especies pertenecientes al subgénero Fibla son elementos faunísticos estacionarios, de expansión moderada.

\section{Fibla (Fibla) hesperica Navás, 1915}

Fibla hesperica Navás, 1915: Navás, 1915d: 25, (d), 25, 26 (mf), 26 (a). Navás, 1915a: 797 (dis, rf). Navás, 1915b: 874 (dis). Navás, 1919: 71 (a, dis, mf, rf). Navás, 1923: 73 (a, dis). Navás, 1928a: 45 (a), 45, 46 (mf), 46 (a, dis). Navás, 1933a: 121 (1st). Navás, 1934a: 7 (dis). Navás, 1934b: 50, 51 (mf), 51 (a, dis). H. Aspöck \& U. Aspöck, 1966b: 108 (dis, gen, rf). H. Aspöck \& U. Aspöck, 1968b: 63 (tx). Lauterbach, 1972: 219 (dis). Willmann, 1994: 188 (a). Monserrat, 1996: 12 (1st). Monserrat \& Triviño, 2013: 15, 22 (lst, rf).

Inocellia (Fibla) hesperica (Navás, 1915): U. Aspöck, 1979: 273 (dis). H. Aspöck et al., 1980a: vol. I: 45 (c), 132 (a, bio, 1, đ̂, +9), 132, 353 (dis), vol. II: 45 (ठ), 168 (a), 8, 11, 260, 263 (dis). Monserrat \& Díaz-Aranda, 1987: 175 (dis). Monserrat \& Triviño, 2013: 15 (lst).

Fibla (Fibla) hesperica Navás, 1915: H. Aspöck et al., 1985a: 387 (pa). H. Aspöck et al., 1991: vol. I: 91 (gen), 94, 97, 98, 483 (pa), 173 (c), 479, 482 (tx), 173, 483, 551, 578, 579 (dis), 482 (a, bio, ô, o, 1, tx), 483 (p), 479, 616 (1st), vol. II: 39, 83 (a), 54 (mf), 306 (ठ), 334 (ㅇ), 356 (1), 529 (dis). U. Aspöck \& H. Aspöck, 1994: 36, 37 (dis). H. Aspöck \& Hölzel, 1996: 45 (dis). H. Aspöck et al., 2001: 57 (tx), 57, 345 (dis). Monserrat \& Papenberg, 2006: 204 (rf). H. Aspöck et al., 2012: 571 (dis). H. Aspöck \& U. Aspöck, 2013: 37 (1st), 146 (gen). Monserrat \& Triviño, 2013: 15, 46 (dis, lst, tx).

= Burcha hispanica Navás, 1928: Navás, 1928a: 49 (d), 49, 51 (mf), 50, 51 (a), 51 (dis), 52 (c). Osorio-Rebellón, 1929: 35 (bio, h, 1), 35-37 (dis, mf), 36, 37 (a), 37 ( ․ ㅇ). Navás, 1933a: 121 (lst). Navás, 1934a: 7 (dis). Navás, 1934b: 56 (c), 57, 58 (a, mf, ð) ), 57, 58 (dis, ㅇ). H. Aspöck et al., 1980a: 132 (sin). Monserrat \& Triviño, 2013: 15 (lst).

= Burcha (Fibla) hispanica Navás, 1928: H. Aspöck \& U. Aspöck, 1966b: 108 (dis, gen, rf). Monserrat \& Triviño, 2013: 15 (lst).

= Fibla (Burcha) hispanica (Navás, 1928): Lauterbach, 1972: 219 (dis). Monserrat \& Triviño, 2013: 15 (lst).

= Fibla pyrenaea Lauterbach, 1972: Lauterbach, 1972: 219 (d, dis), 220-224 (mf), 221 (a), 221-224, 226 (す), 224, 225 (bio), 228 (tx). H. Aspöck et al., 1980a: 132 (sin). Monserrat \& Triviño, 2013: 15 (lst).

Inocellia maclachlani Albarda, 1891: Navás, 1905a: 38 (mf). Navás, 1908: 36 - (ie). Monserrat \& Triviño, 2013: 15 (lst). 
TIPO: Holotipo depositado en el Museo Municipal de Barcelona (falta el abdomen aunque probablemente se trate de una ${ }_{+}$).

LOCALIDAD TÍPICA: San Fiel (Portugal).

MATERIAL ESTUDIADO: ESPAÑA: Ávila: Arevalillo, 30TTK9995, $1.131 \mathrm{~m}, 14 . \mathrm{VI} .1977,1$ đे sobre Pinus sylvestris, V. J. Monserrat (VM). Cuenca: La Serna, Tragacete, 30TXK0262, $1.250 \mathrm{~m}, 30 . \mathrm{VI} .1985,1$ q sobre P. sylvestris, V. J. Monserrat (VM). Granada: Dehesa del Generalife, 23.IV.2012, 1 ô, M. Morente (VM), Ferreira, 30SVG9714, 398 m, 8.V.2011, 1 우 sobre $P$. halepensis, V. Treviño (VM). Madrid: Madrid, Ciudad Universitaria, 14.V.2013, 1 §, M. Villatori, Madrid, Parque del Oeste, 13.V.2013, 1 ठ errante, V. J. Monserrat (VM). Salamanca: Calzadilla, 29TQE1036, 760 m, 27.V.1976, 1 , A. Hernández (VM). Segovia: Rio Voltoya, 2.VI.1981, $1 \delta^{\curvearrowright}\left(\mathrm{a}^{*}\right)$ sobre P. pinaster, V. J. Monserrat (VM). Toledo: Puerto de San Vicente, 30SUJ1877, 800 m, 26.V.1978, 1 ㅇ sobre P. pinaster, V. J. Monserrat (VM).

MORFOLOGÍA EXTERNA: Es una especie de tamaño desde mediano hasta grande. La longitud del ala anterior es de entre $8.5-11.5 \mathrm{~mm}$ en $\operatorname{los} \widehat{\partial} \widehat{\partial} \mathrm{y}$ 10-15 mm en las 우 우 (figs. 39, 42). La cabeza presenta un dibujo ocre. El clípeo es ocre y el labro es pardo oscuro. El torulus en el ô está levemente ensanchado en su cara interna. El escapo y el pedicelo son pardo oscuro con los bordes algo más claros. El flagelo es pardo oscuro, aclarándose hacia el extremo. El pronoto presenta un dibujo pardo en el $\delta$ y amarillo en las 9 . Los apéndices locomotores presentan las coxas de coloración parda oscuro. Los trocánteres y fémures son pardos. El resto de los artejos son ocres. En el abdomen los terguitos y esternitos son de coloración parda con una banda posterior más clara.

SEGMENTOS GENITALES: En los $\widehat{\partial} \widehat{\partial}$ el gonarco tiene el extremo bifurcado (figs. 78-80). En las 우우 la subgenitalia no presenta placas esclerotizadas y la interna es muy característica (figs. 123, 140).

BIOLOGÍA: Se han hallado imagos sobre todo sobre Pinus. Los biotopos típicos son pinares despejados, incluso parques urbanos (con coníferas), existiendo una preferencia por zonas más templadas de barrancos pedregosos con árboles solitarios. Otras especies del género, dentro de sus áreas de distribución son especies eurioicas, pero existe una tendencia a colonizar biotopos templados. Su distribución vertical es entre los 398-1.250 m, en otras especies próximas la distribución vertical comprende entre los 150-1.800 m. Las zonas de distribución suelen ser muy localizadas y las densidades de población en la mayoría de los casos son bajas, pero se registran en gran número individuos en fase de larva. Se ha citado como parásito: Mermithidae gen. sp. (Sistema Ibérico) y parasitoide: Nemeritis specularis specularis (Alicante) y Nemeritis caudata (Sistema Ibérico).

FENOLOGÍA: La actividad de los imagos es monovoltina, estacionaria, mayoritariamente primaveral, transcurriendo entre los meses IV-VII.
VARIABILIDAD: Al margen de las variaciones en el tamaño la variabilidad es escasa.

ESTADIOS PREIMAGINALES: Las larvas son corticícolas, tanto de coníferas como de árboles planifolios, no parecen mostrar preferencia aparente por ningún tipo de planta soporte, aunque existe una predilección clara por biotopos cálidos. El periodo de desarrollo es de al menos tres años, siendo frecuentes tiempos más largos de hasta seis años (en el género Reisserella el periodo es de dos años). La última hibernación es en fase de larva, y por tanto son ciclos vitales de tipo I (fig. 18). El estadio de pupa se mantiene entre 10-20 días.

La figura dorsal se encuentra muy expandida, ocupando la mayor parte del segmento, pudiendo aparecer zonas más claras de diverso tamaño en la parte anterior, lateral y caudal y por tanto son estas zonas las que definen el dibujo (fig. 155). La banda dorsal es muy delgada. En la mayoría de los casos transcurre de forma continuada (fig. 155). La figura lateral está muy expandida y se encuentra unida a la dorsal mediante pequeñas zonas mas claras. La figura ventral está formada por una zona media ancha y dos bandas laterales (las larvas de las tres especies citadas son muy parecidas entre sí y probablemente no se puedan diferenciar en muchas ocasiones con seguridad). Morfología y aspecto de la larva en figura 155 y en H. Aspöck et al. (1991).

DISTRIBUCIÓN: España y Portugal con aparente ausencia en el noroeste de la Península. Elemento faunístico monocéntrico, atlantomediterráneo (fig. 171).

DISCUSIÓN: Fibla hesperica no presenta similitud con ninguna otra especie ibérica, de las que se diferencia de inmediato. Es la especie más cercana a Fibla maclachlani, presentando un gran parecido morfológico, aunque existen diferencias morfológicas en su genitalia como son la forma de los gonocoxitos, serratulum, gonarco, arceso, o hipandrio interno en los ô $\widehat{o}$ o la existencia o no de placas esclerotizadas en la subgenitalia de las $\bigcirc$ ㅇ. Esta especie ha sido citada junto a Hispanoraphida castellana, Atlantoraphidia maculicollis, Harraphidia laufferi, Subilla aliena, Ohmella baetica, Ohmella bolivari y Ohmella postulata.

\section{Agradecimientos}

Esta contribución forma parte del Proyecto coordinado de I + D $+\mathrm{i}$ Fauna Ibérica (Neuroptera) CGL2010-22267-C07-05. Deseamos agradecer a los museos e instituciones citadas el habernos permitido estudiar ejemplares de sus colecciones, y a los autores y compañeros citados en el material estudiado por el envío de ejemplares de sus colecciones particulares o de sus centros de investigación. También deseamos manifestar nuestro sincero agradecimiento a R. A. Pantaleoni por su apoyo y sus comentarios, y a las autoridades de las diferentes Comunidades Autónomas por facilitarnos los correspondientes permisos de muestreos para la toma de datos en los espacios protegidos visitados. 

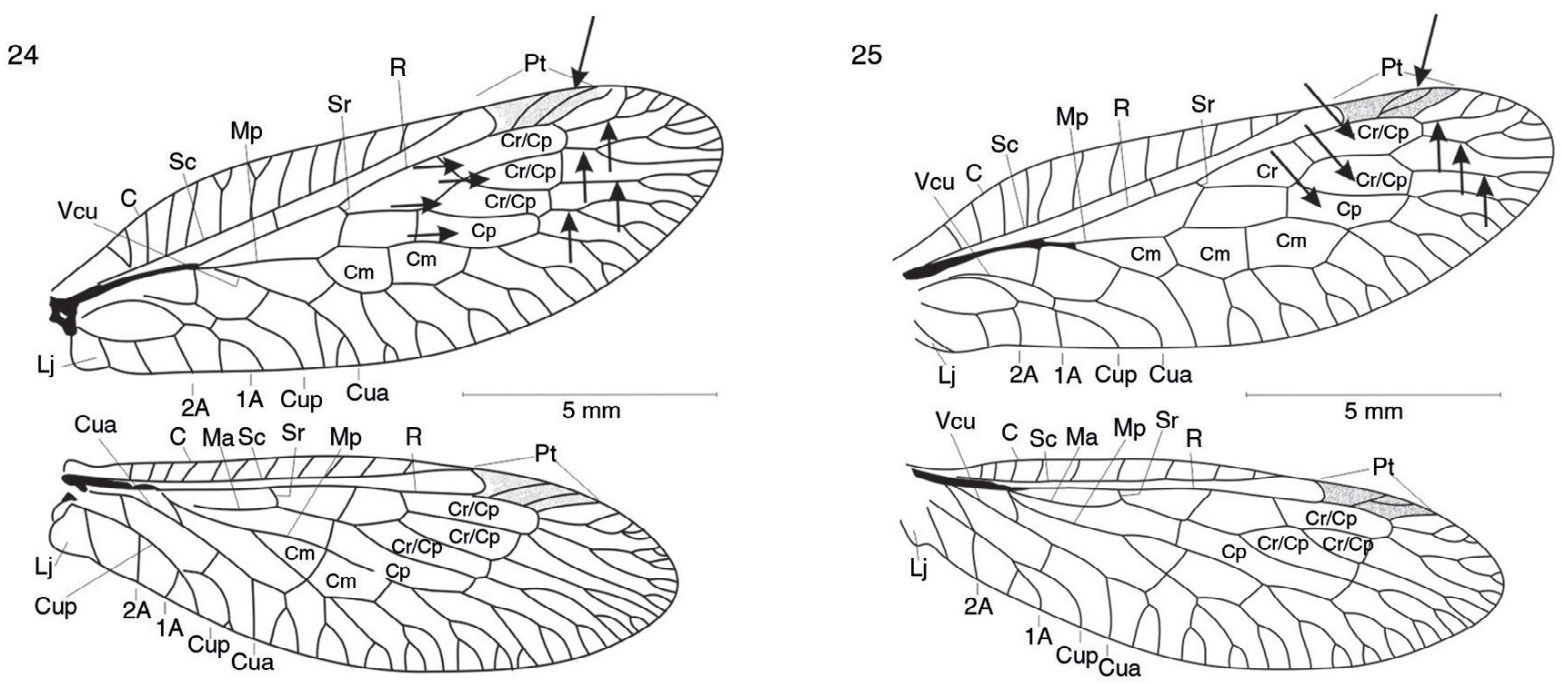

26

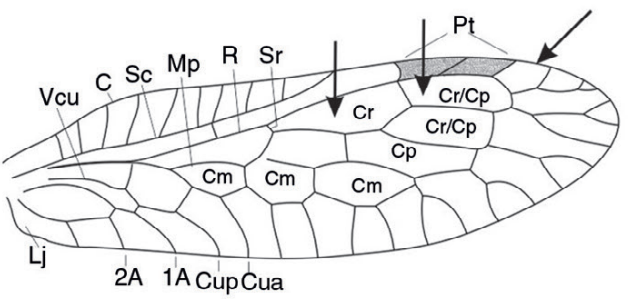

27
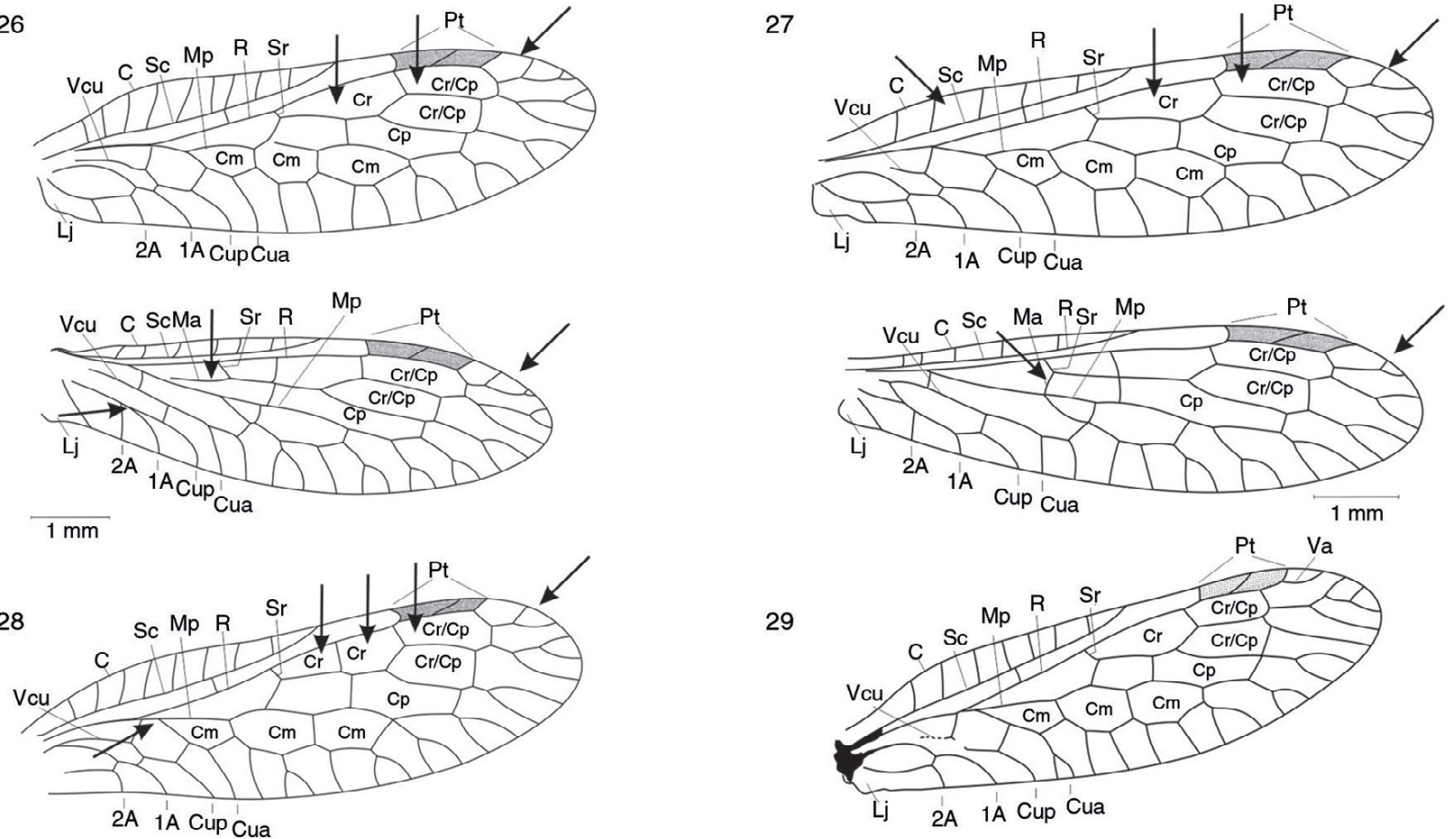

2A 1'A Gúp'Cua
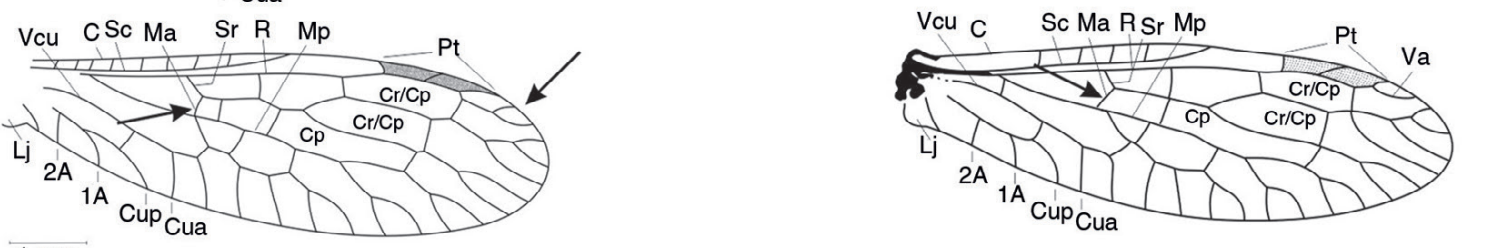

$1 \mathrm{~mm}$

$1 \mathrm{~mm}$

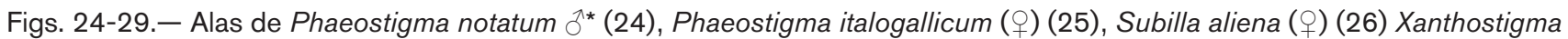
aloysianum (+) (27), Xanthostigma corsicum (+) (28) y Atlantoraphidia maculicollis (o)* (29). 1A: $1^{\text {a }}$ venal anal, $2 \mathrm{~A}: 2^{\mathrm{a}}$ vena anal, C: vena costal, Cm: celda media, Cp: celda postigmal, Cr: celda radial, Cua: cúbito anterior, Cup: cúbito posterior, Lj: lóbulo yugal, Ma: mediana anterior, Mp: mediana posterior, Pt: pterostigma, R: radio, Sc: vena subcostal, Sr: sector del radio, Va: vena apical, Vcu: vena cubital. * Adaptado de H. Aspöck et al. (1991).

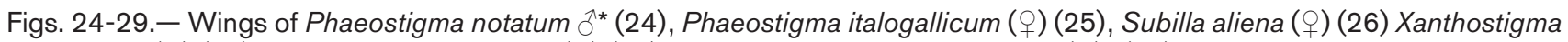
aloysianum (P) (27), Xanthostigma corsicum (P) (28) and Atlantoraphidia maculicollis ( ()$^{\star}(29)$. 1A: 1st anal vein, 2A: $2 \mathrm{nd}$ anal vein, C: costal vein, $\mathrm{Cm}$ : median cell, $\mathrm{Cp}$ : postigmal cell, Cr: radial celilla, Cua: anterior cubitus, Cup: posterior cubitus, Lj: yugal lobe, Ma: anterior median, Mp: posterior median, Pt: pterostigme, R: radius, Sc: subcostal vein, Sr: radial sector, Va: apical vein, Vcu: cubital vein. ${ }^{*}$ Adapted from H. Aspöck et al. (1991). 
30

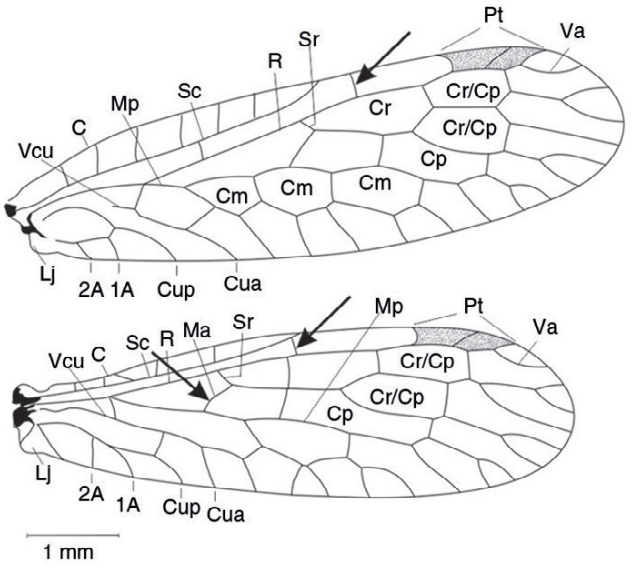

32
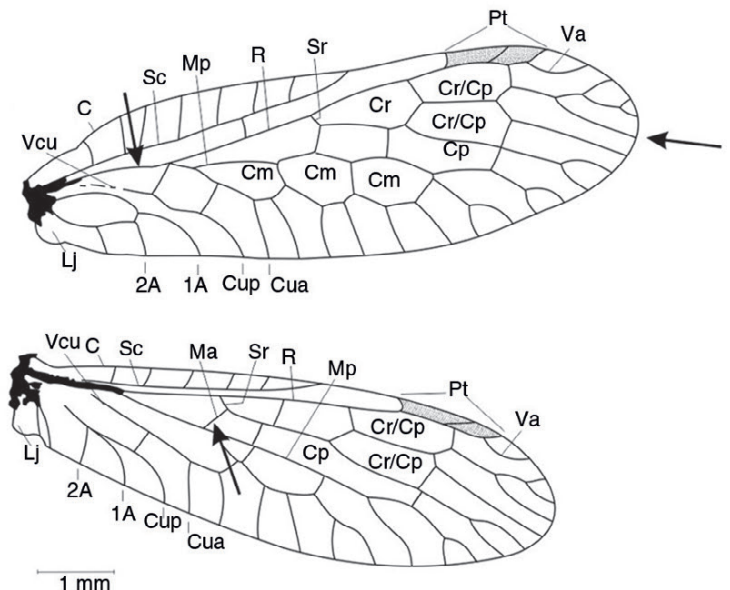

34
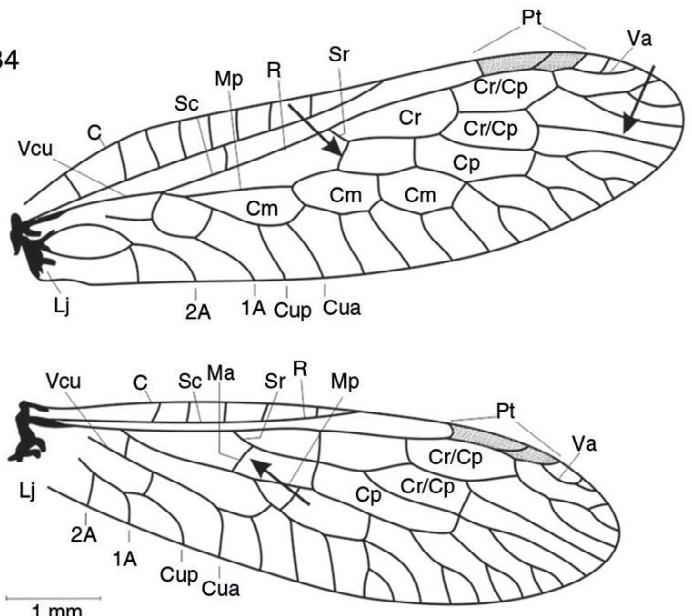

31

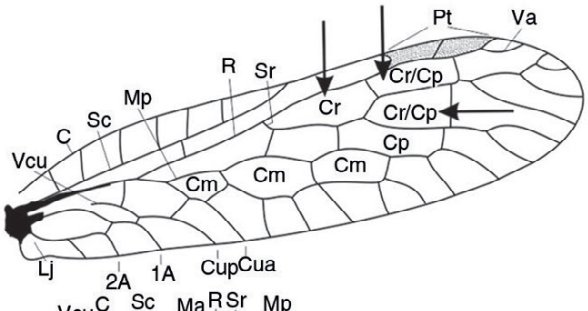

$\mathrm{Vcu}^{2 \mathrm{~A}} \mathrm{Sc} \mathrm{MaRSr} \mathrm{Mp}$

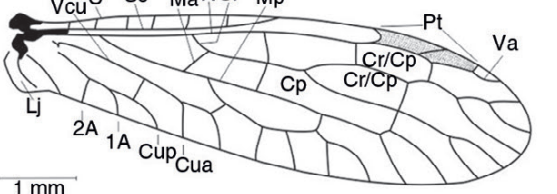

33
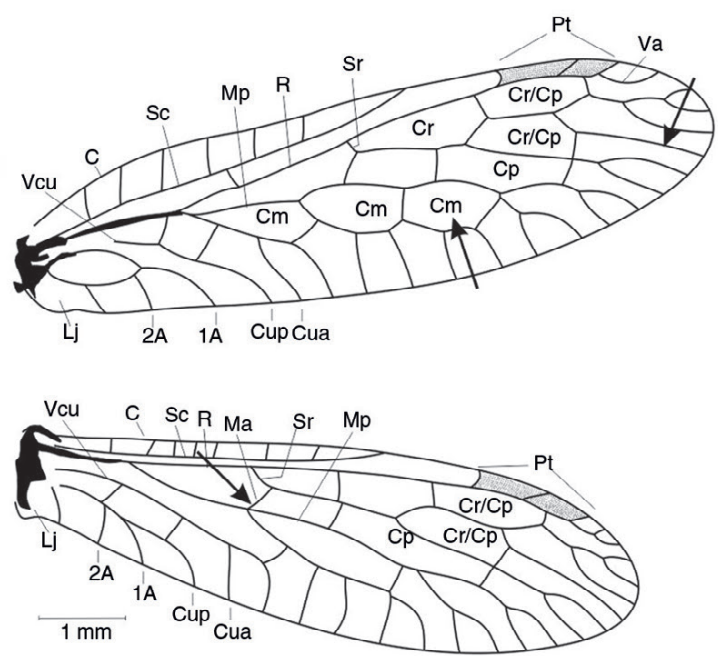

35
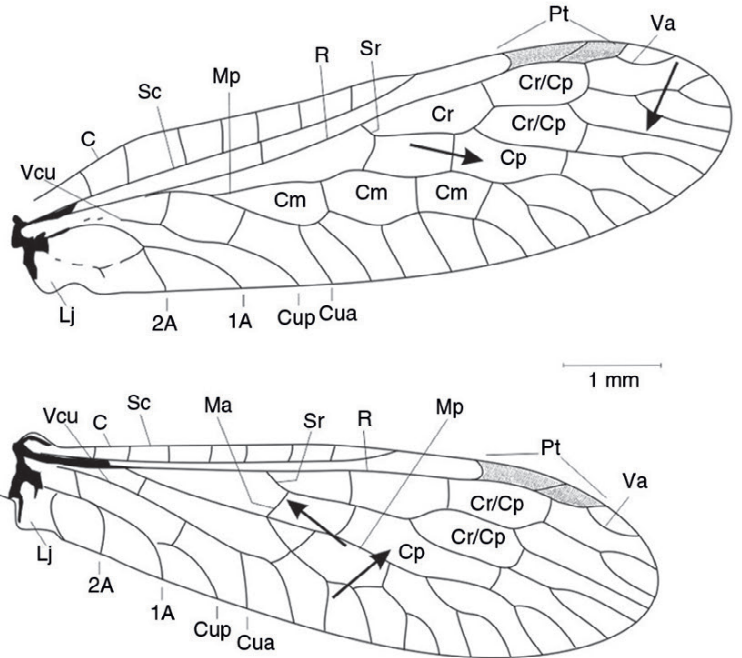

Figs. 30-35.- Alas de Harraphidia (H.) subdesertica (holotipo $\left.{ }^{\jmath}\right)(30)$, Harraphidia laufferi $(9)^{\star}(31)$, Hispanoraphidia castellana

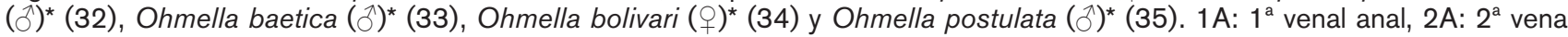
anal, C: vena costal, $\mathrm{Cm}$ : celda media, Cp: celda postigmal, Cr: celda radial, Cua: cúbito anterior, Cup: cúbito posterior, Lj: lóbulo yugal, Ma: mediana anterior, Mp: mediana posterior, Pt: pterostigma, R: radio, Sc: vena subcostal, Sr: sector del radio, Va: vena apical, Vcu: vena cubital. * Adaptadas de H. Aspöck et al. (1991).

Figs. 30-35.- Wings of Harraphidia (H.) subdesertica (holotype §`), (30), Harraphidia laufferi (+)* (31), Hispanoraphidia

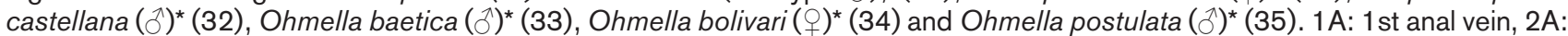
2nd anal vein, C: costal vein, Cm: median cell, Cp: postigmal cell, Cr: radial celilla, Cua: anterior cubitus, Cup: posterior cubitus, Lj: yugal lobe, Ma: anterior median, Mp: posterior median, Pt: pterostigme, R: radius, Sc: subcostal vein, Sr: radial sector, Va: apical vein, Vcu: cubital vein. * Adapted from H. Aspöck et al. (1991). 

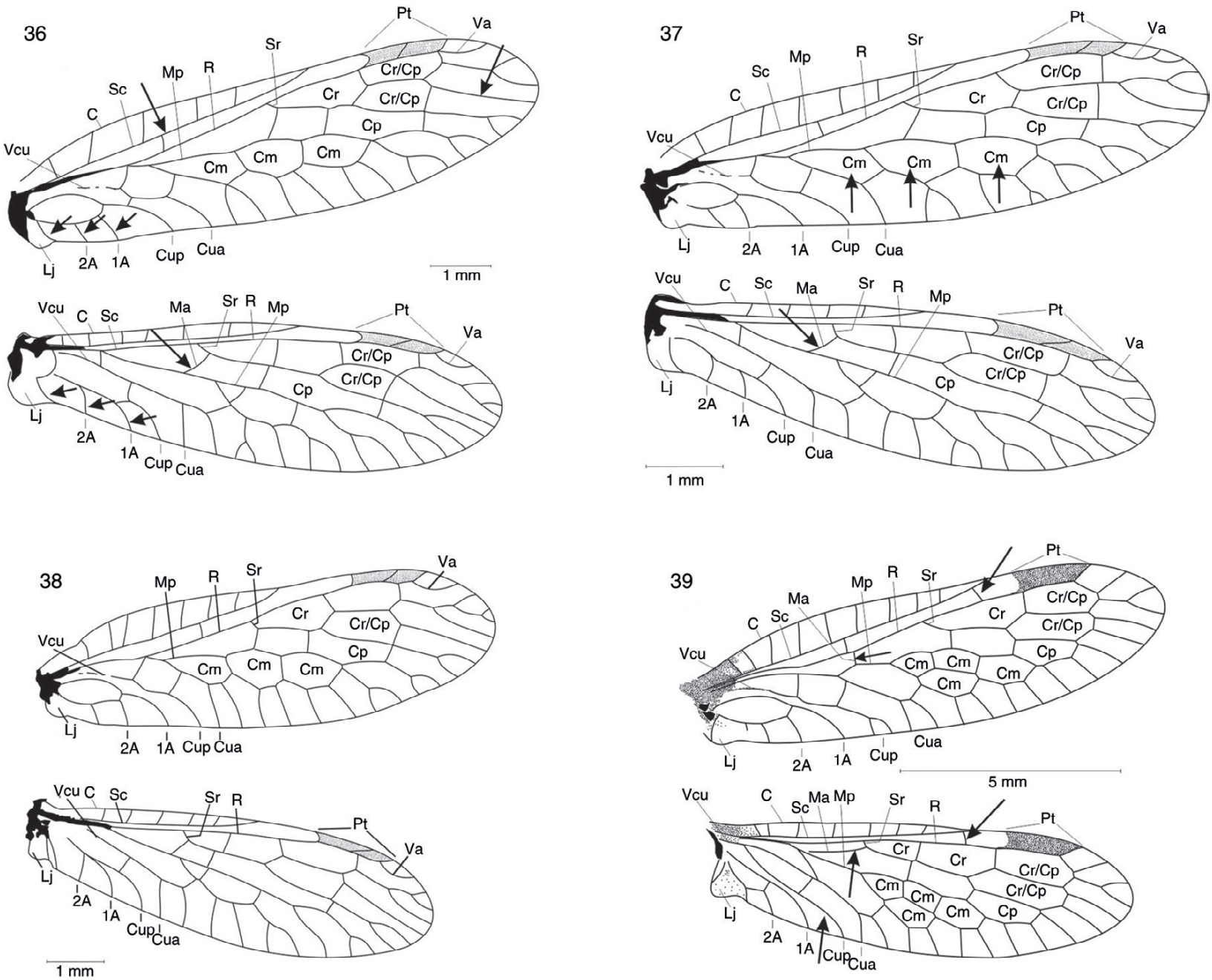

40

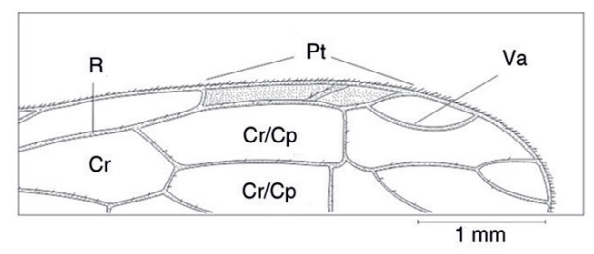

41
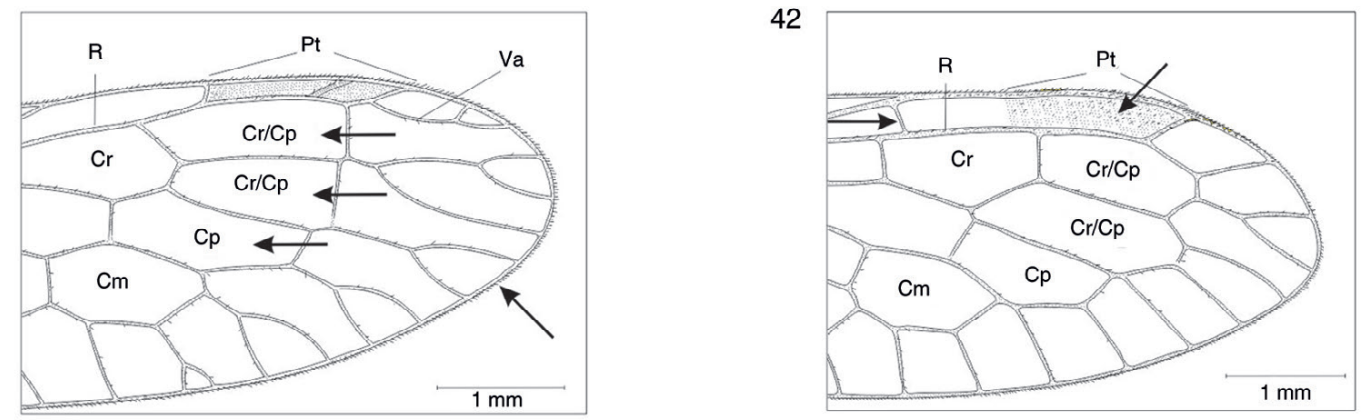

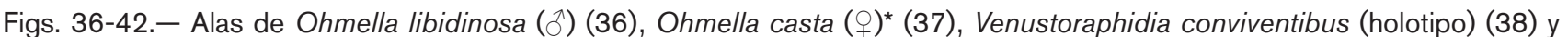
Fibla (F.) hesperica (+) (39). Detalle de la región del pterostigma () del ala anterior de Ohmella casta* $(40,41)$ y de Fibla (F.) hesperica (42). 1A: $1^{a}$ venal anal, 2A: $2^{a}$ vena anal, C: vena costal, Cm: celda media, Cp: celda postigmal, Cr: celda radial, Cua: cúbito anterior, Cup: cúbito posterior, Lj: lóbulo yugal, Ma: mediana anterior, Mp: mediana posterior, Pt: pterostigma, R: radio, Sc: vena subcostal, Sr: sector del radio, Va: vena apical, Vcu: vena cubital. * Adaptadas de H. Aspöck et al. (1991).

Figs. 36-42.- Wings of Ohmella libidinosa (ふ઼) (36), Ohmella casta (†)* (37), Venustoraphidia conviventibus (holotype) (38) y Fibla (F.) hesperica (O) (39). Particular of the fore wings pterostigmatic area ( $($ ) of Ohmella casta* $(40,41)$ and Fibla (F.) hesperica (42). 1A: 1st anal vein, 2A: 2nd anal vein, C: costal vein, Cm: median cell, Cp: postigmal cell, Cr: radial celilla, Cua: anterior cubitus, Cup: posterior cubitus, Lj: yugal lobe, Ma: anterior median, Mp: posterior median, Pt: pterostigme, R: radius, Sc: subcostal vein, Sr: radial sector, Va: apical vein, Vcu: cubital vein. * Adapted from H. Aspöck et al. (1991). 
43

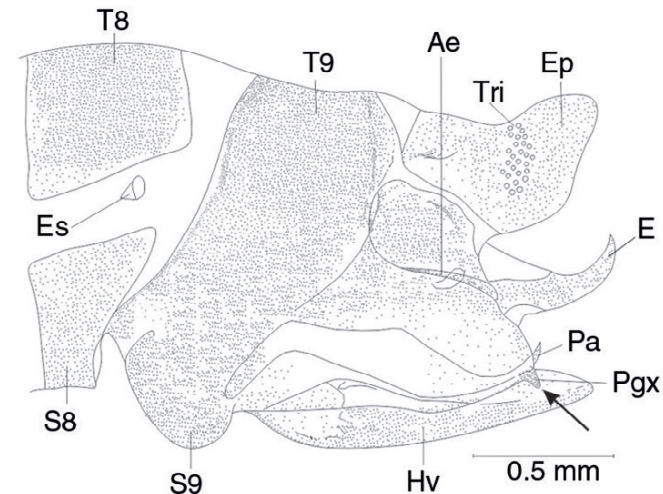

S9

44

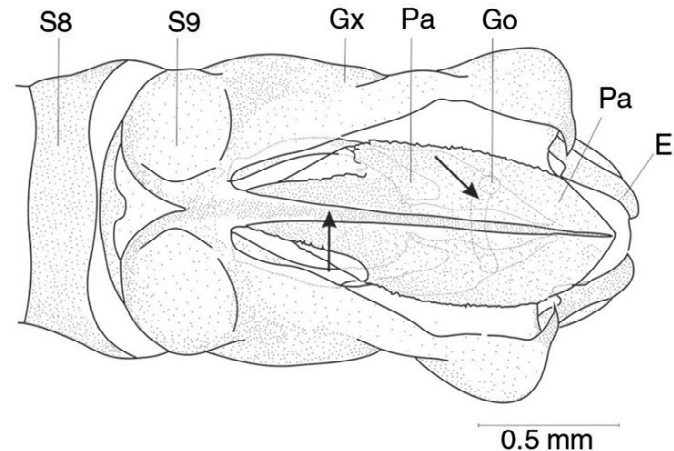

Ep

45
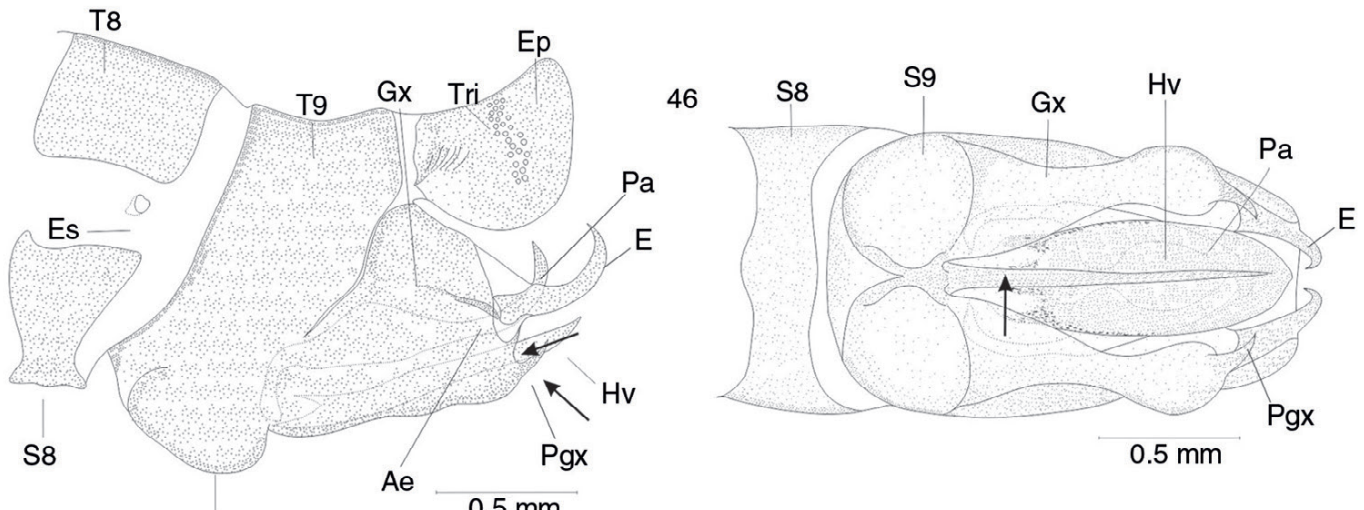

47

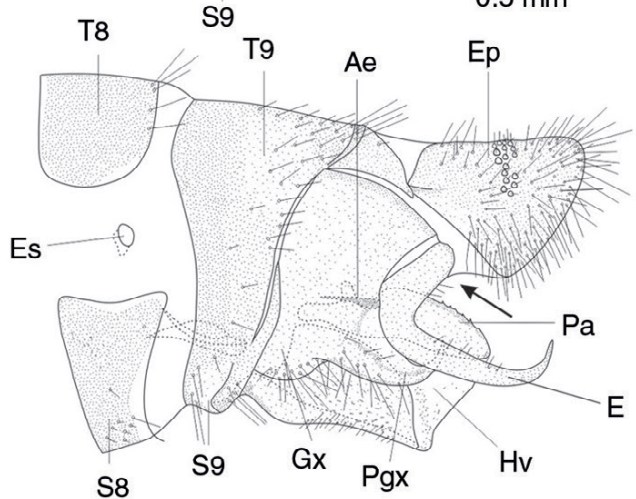

48
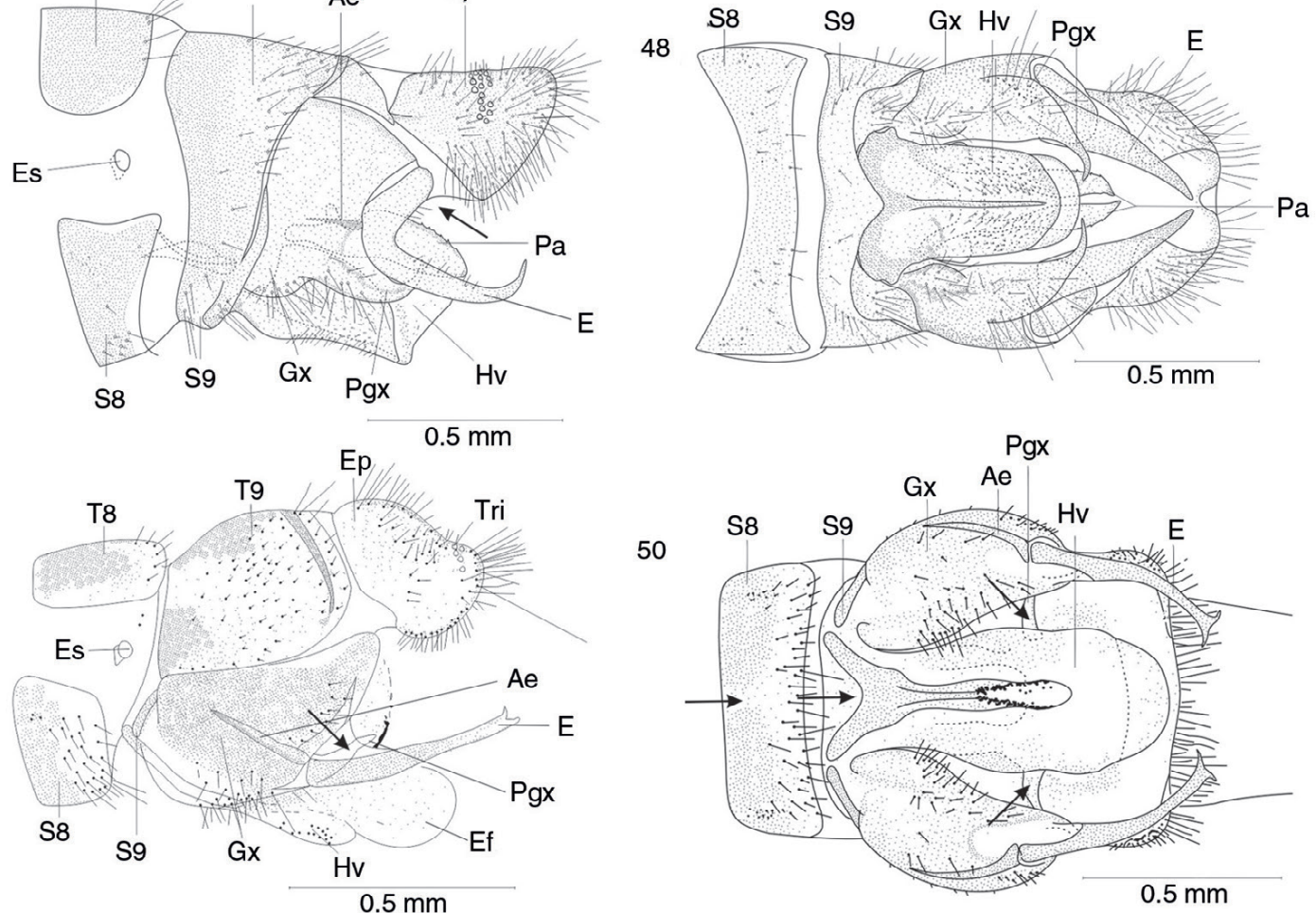

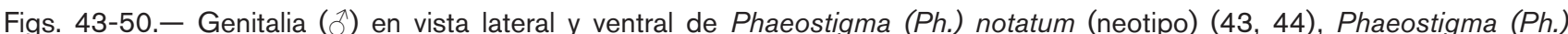
italogallicum (holotipo) (45-46), Subilla alienai (47-48) y Xanthostigma aloysianum (49-50). Ae: apodema del estilo, Agx: apodema del gonocoxito, E: estilo, Ef: endofalo, Ep: ectoprocto, Es: espiráculo, Hi: hipandrio, Hv: hipovalva, Gx: gonocoxito, Pa: parámero, Pgx: proceso del gonocoxito, S: esternito, T: terguito, Tr: tricobotrios. Adaptadas de H. Aspöck et al. (1991).

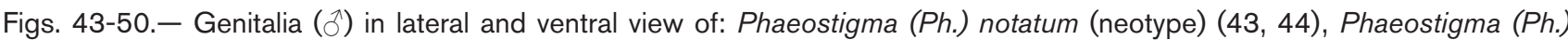
italogallicum (holotype) $(45,46)$, Subilla aliena (47-48) y Xanthostigma aloysianum (49-50). Ae: stilus apodeme, Agx: gonocoxite apodeme, E: estilus, Ef: endofalus, Ep: ectoproct, Es: spiracle, Hi: hypandrium, Hv: hypovalve, Gx: gonocoxite, Pa: paramera, Pgx: gonocoxite processus, S: sternite, T: tergite, Tr: trichobotria. Adapted from H. Aspöck et al. (1991). 
51

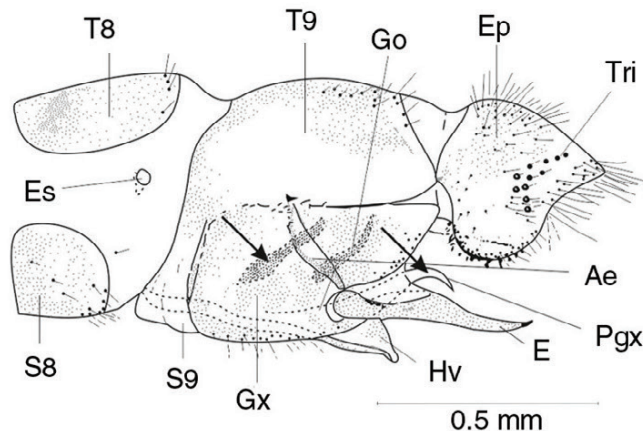

53
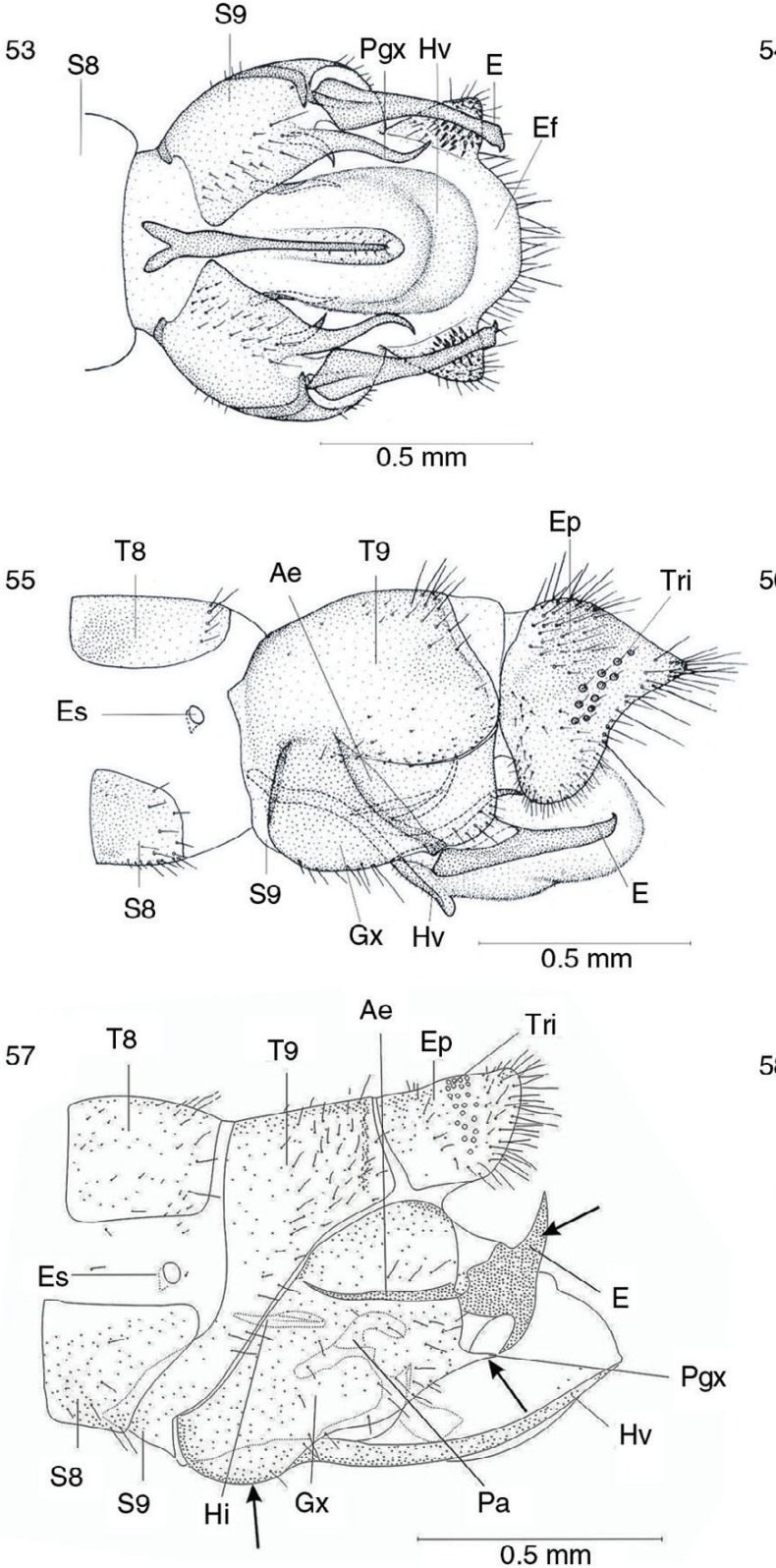

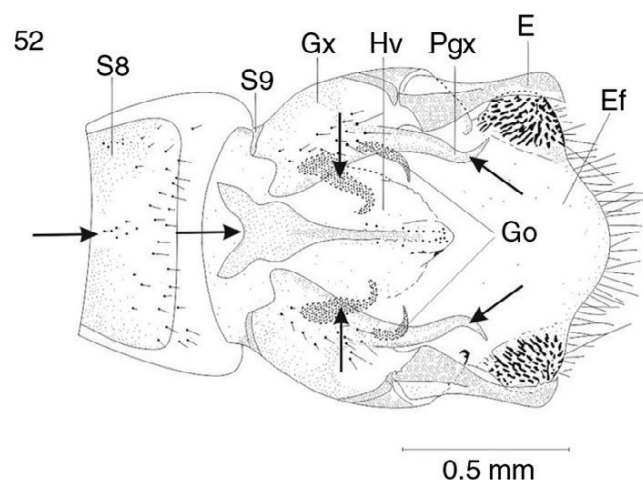

54

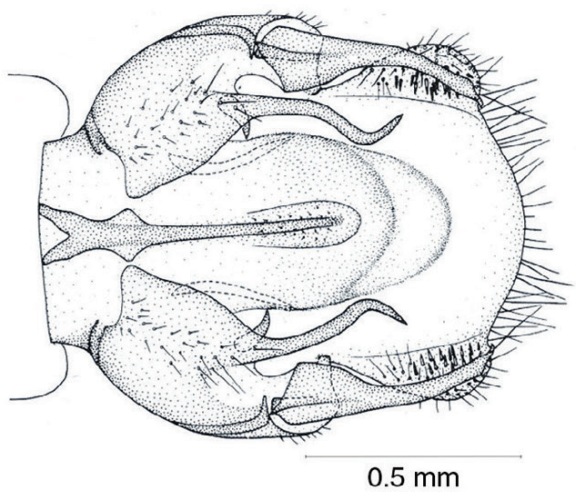

56

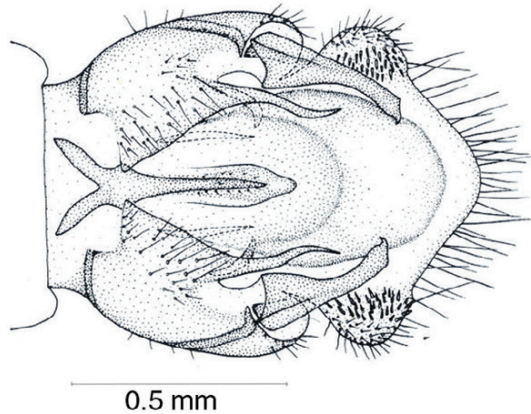

58

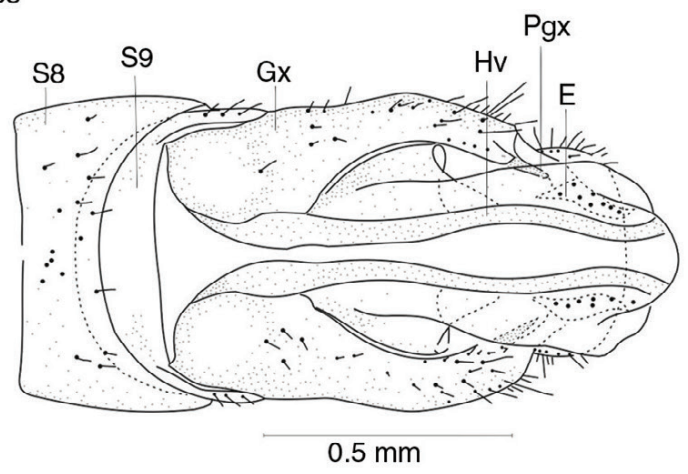

Figs. 51-58.- Genitalia ( $\left.{ }^{(}\right)$en vista ventral de Xanthostigma corsicum de España $(51,52)$, de Sicilia $(53)$ y de Italia peninsular

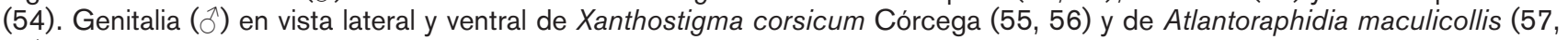
58). Ae: apodema del estilo, E: estilo, Ef: endofalo, Ep: ectoprocto, Es: espiráculo, Hi: hipandrio, Hv: hipovalva, Gx: gonocoxito, Pa: parámero, Pgx: proceso del gonocoxito, S: esternito, T: terguito, Tr: tricobotrios. Adaptadas de H. Aspöck et al. (1991).

Figs. 51-58.- Genitalia ( $(3)$ in ventral view of Xanthostigma corsicum from Spain $(51,52)$, Sicily (53) and Peninsular Italy (54). Genitalia $(\widehat{)})$ in lateral and ventral view of Xanthostigma corsicum from Corsica $(55,56)$ and Atlantoraphidia maculicollis $(57$, 58). Ae: stilus apodeme, E: estilus, Ef: endofalus, Ep: ectoproct, Es: spiracle, Hi: hypandrium, Hv: hypovalve, Gx: gonocoxite, Pa: paramera, Pgx: gonocoxite processus. S: sternite, T: tergite, Tr: trichobotria. Adapted from H. Aspöck et al. (1991). 


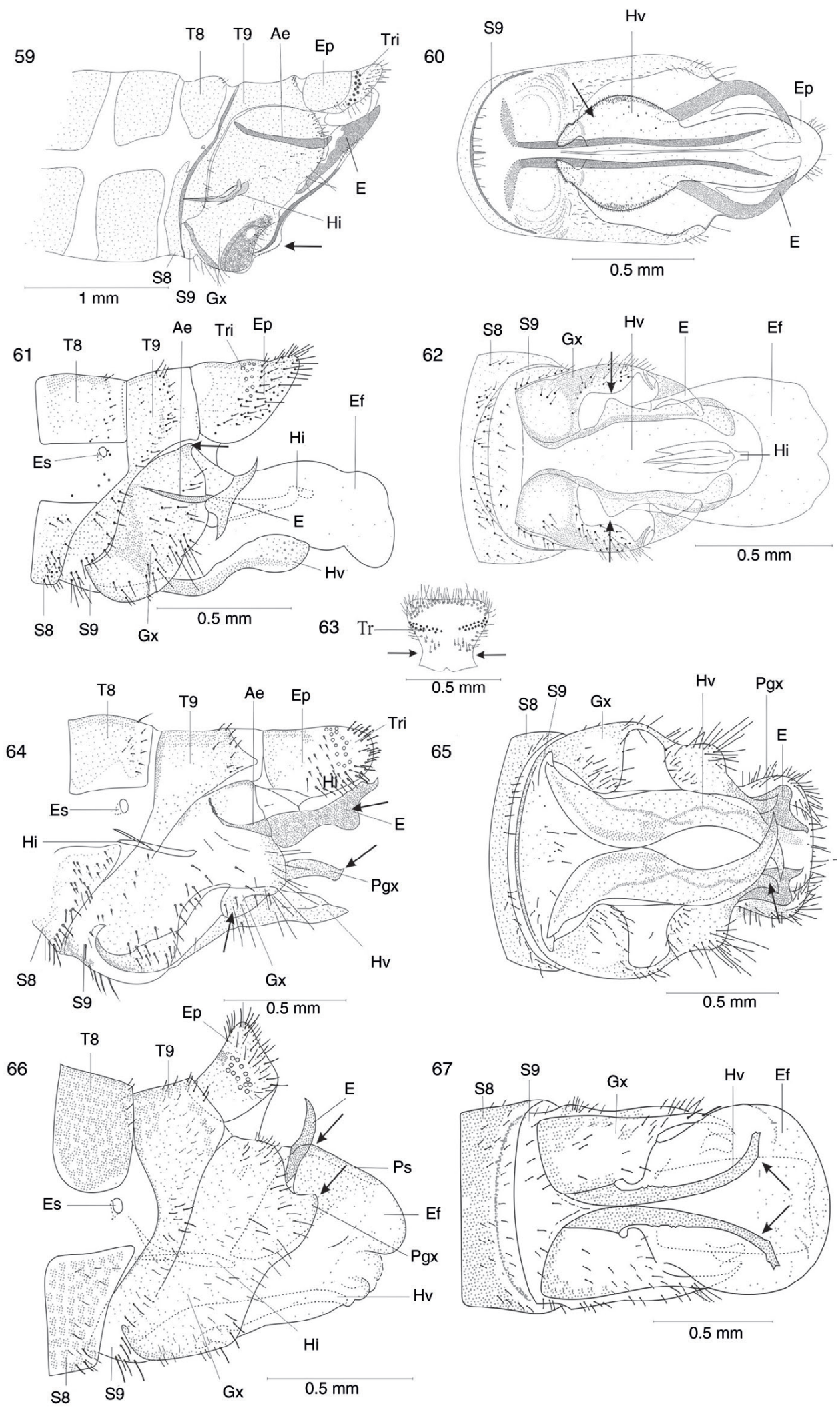

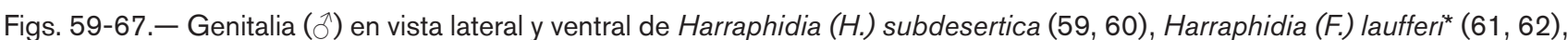
Hispanoraphidia castellana* $(64,65)$ y de Ohmella baetica* $(66,67)$. Ectoprocto en vista dorsal de Hispanoraphidia castellana (63). Ae: apodema del estilo, E: estilo, Ef: endofalo, Ep: ectoprocto, Es: espiráculo, Hi: hipandrio, Hv: hipovalva, Gx: gonocoxito, Pa: parámero, Pgx: proceso del gonocoxito, S: esternito, T: terguito, Tr: tricobotrios. * Adaptadas de H. Aspöck et al. (1991).

Figs. 59-67.- Genitalia ( $(1)$ in lateral and ventral view of Harraphidia (H.) subdesertica $(59,60)$, Harraphidia (F.) laufferi* (61, 62), Hispanoraphidia castellana* $(64,65)$ and Ohmella baetica* $(66,67)$. Ectoproct in dorsal view of Hispanoraphidia castellana (63). Ae: stilus apodeme, E: estilus, Ef: endofalus, Ep: ectoproct, Es: spiracle, Hi: hypandrium, Hv: hypovalve, Gx: gonocoxite, Pa: paramera, Pgx: gonocoxite processus. S: sternite, T: tergite, Tr: trichobotria. * Adapted from H. Aspöck et al. (1991). 

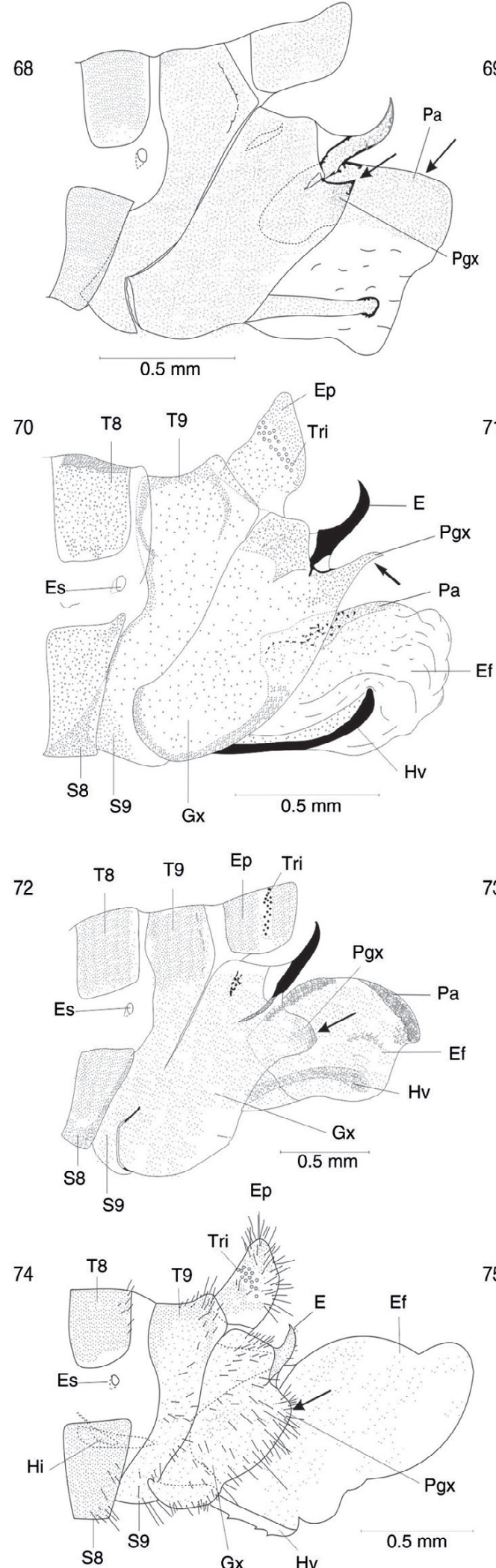

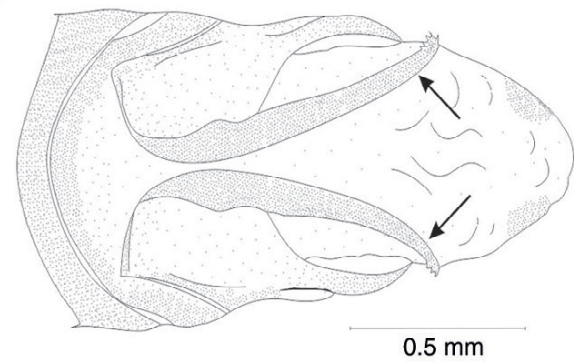

71
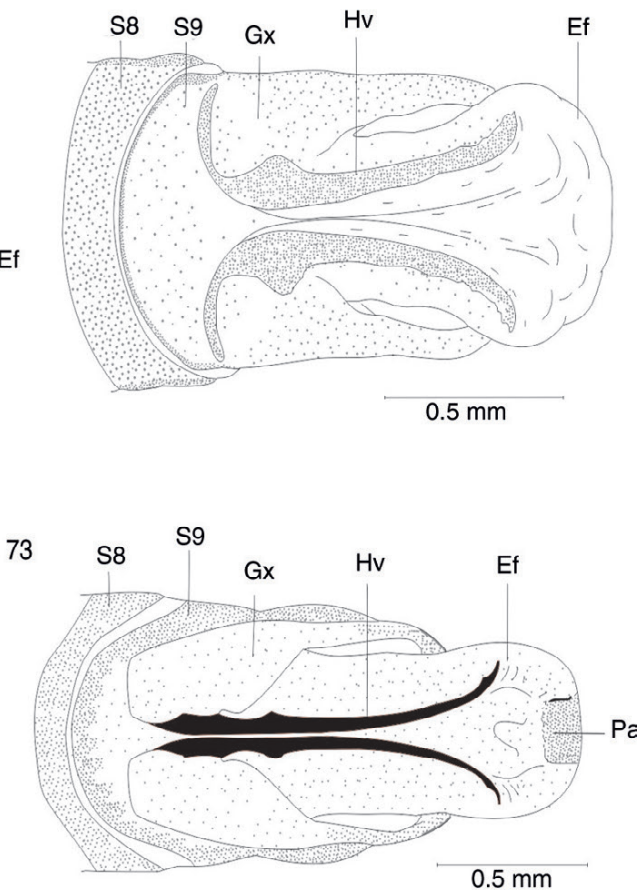

75

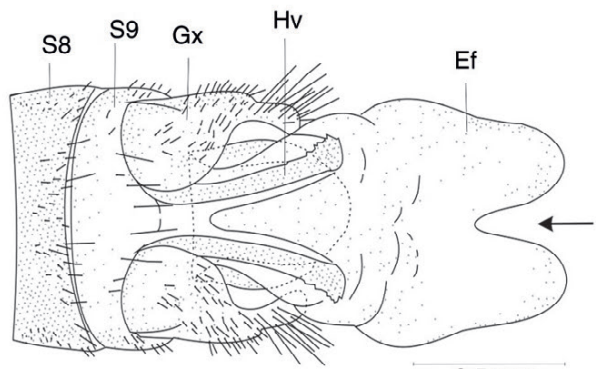

$0.5 \mathrm{~mm}$

Figs. 68-75.- Genitalia ( $(\hat{)})$ en vista lateral y ventral de Ohmella bolivari $(68,69)$, Ohmella postulata (70, 71), Ohmella libidinosa $(72,73)$ y Ohmella casta $(74,75)$. Ae: apodema del estilo, E: estilo, Ef: endofalo, Ep: ectoprocto, Es: espiráculo, Hi: hipandrio, Hv: hipovalva, Gx: gonocoxito, Pa: parámero, Pgx: proceso del gonocoxito, S: esternito, T: terguito, Tr: tricobotrios. Adaptadas de H. Aspöck et al. (1991).

Figs. 68-75.- Genitalia ( $(\lambda)$ in lateral and ventral view of Ohmella bolivari $(68,69)$, Ohmella postulata $(70,71)$, Ohmella libidinosa $(72,73)$ y Ohmella casta $(74,75)$. Ae: stilus apodeme, E: estilus, Ef: endofalus, Ep: ectoproct, Es: spiracle, Hi: hypandrium, Hv: hypovalve, Gx: gonocoxite, Pa: paramera, Pgx: gonocoxite processus. S: sternite, T: tergite, Tr: trichobotria. Adapted from H. Aspöck et al. (1991). 


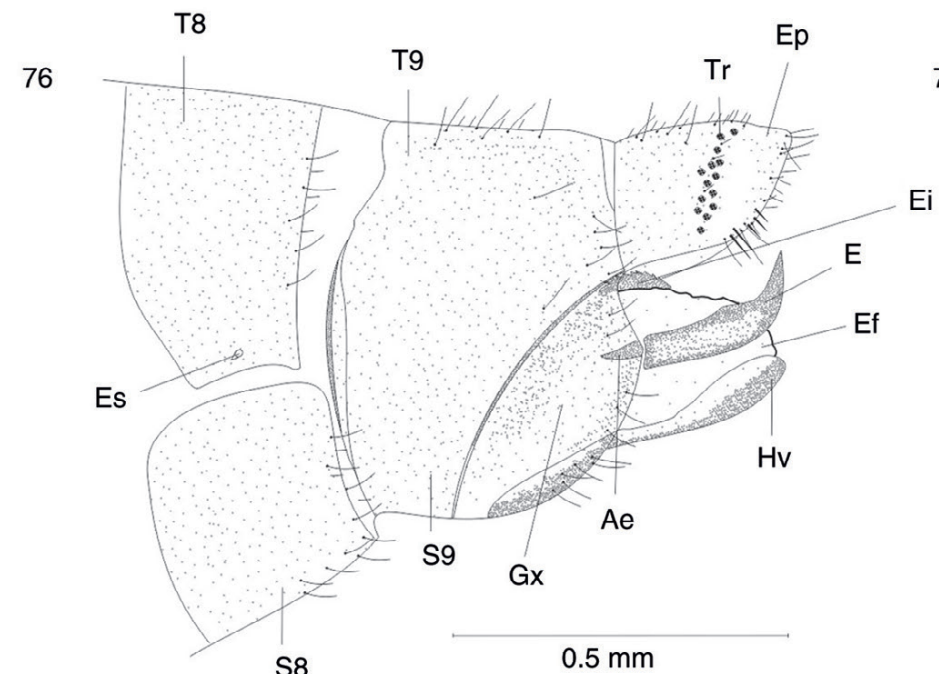

77

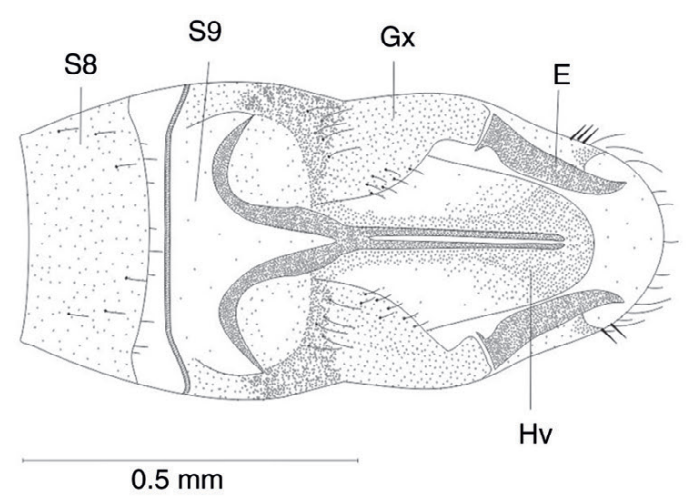

S8

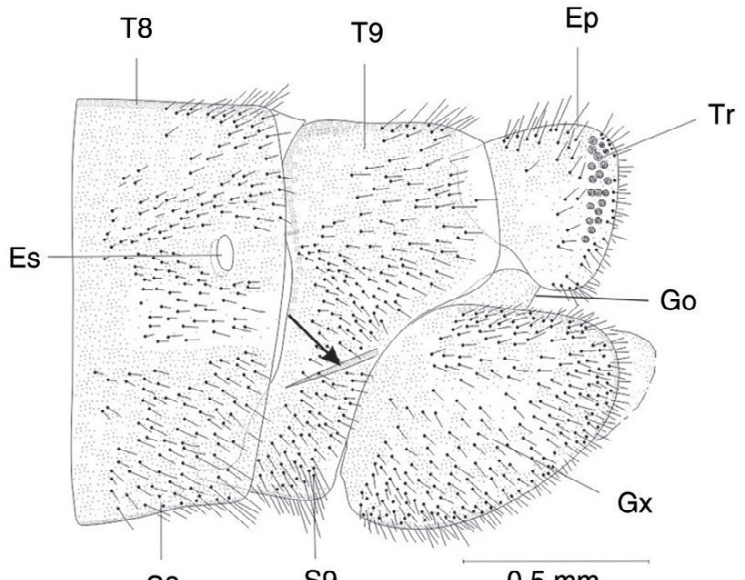

$\operatorname{Tr}$

79

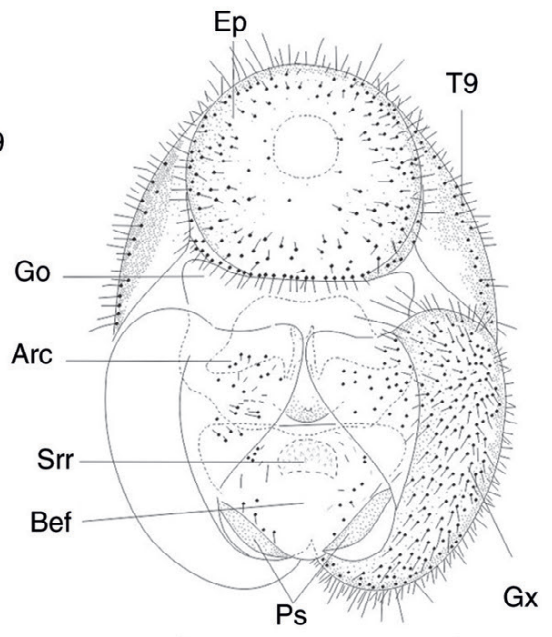

S8

S9

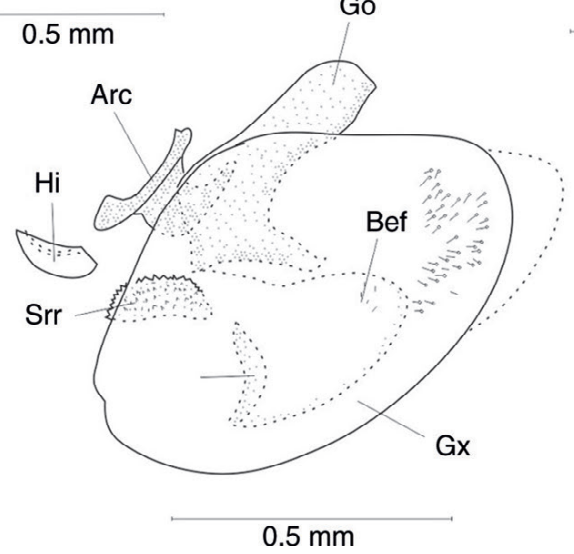

80

$0.5 \mathrm{~mm}$

Figs. 76-80.- Genitalia (§) en vista lateral y ventral de Venustoraphidia conviventibus (holotipo) (76, 77). Genitalia (ð) en vista lateral y caudal de Fibla (F.) hesperica* $(78,79)$. Detalle de los escleritos internos ( $\left.{ }^{\Uparrow}\right)$ de Fibla (F.) hesperica* (80). Arc: arceso, Bef: base del endofalo, E: estilo, Ef: endofalo, Ep: ectoprocto, Es: espiráculo, Go: gonarco, Gx: gonocoxito, Hi: hipandrio, Hv: hipovalva, Pgx: proceso del gonocoxito, Ps: pseudoestilo, Srr: serratulum, S: esternito, T: terguito, Tr: tricobotrios. ${ }^{*}$ Adaptadas de H. Aspöck et al. (1991).

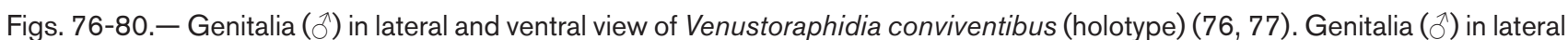
and caudal view of Fibla (F.) hesperica* $(78,79)$. Particular of inner sclerites ( $\left.{ }^{\star}\right)$ of Fibla (F.) hesperica* (80). Arc: arcessus, Bef: endofalus basis, E: stilus, Ef: endofalus, Ep: ectoproct, Es: spiracle, Go: gonarcus, Gx: gonocoxite, Hi: hypandrium, Hv: hypovalve, Pgx: gonocoxite processus. Ps: pseudoestilus, Srr: serratulum, S: sternite, T: tergite, Tr: trichobotria. * Adapted from H. Aspöck et al. (1991). 
81

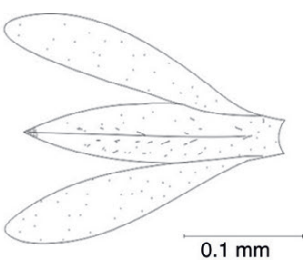

82
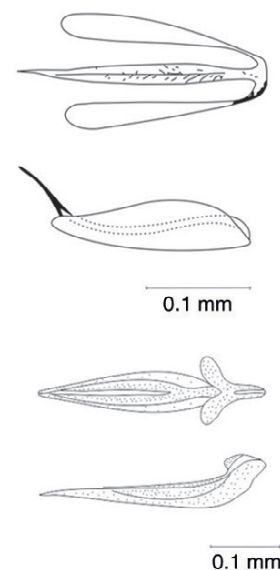

90

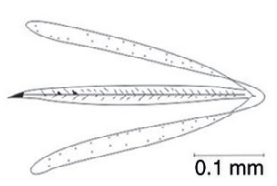

83

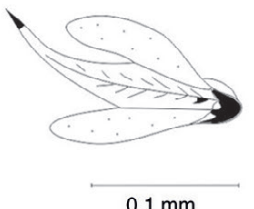

$0.1 \mathrm{~mm}$

87

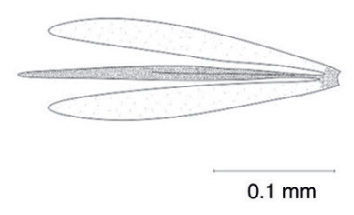

91

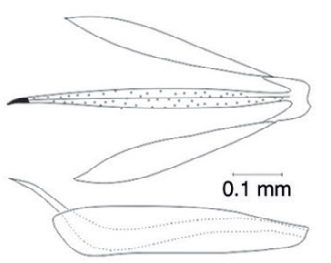

84

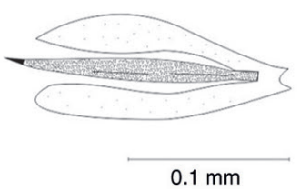

88

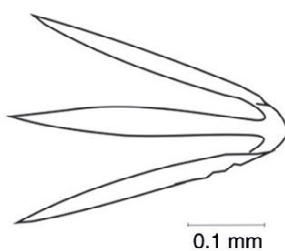

$0.1 \mathrm{~mm}$

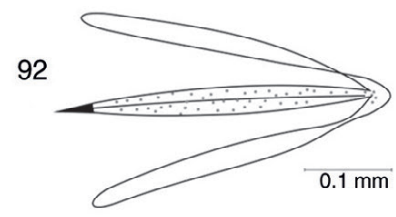

93

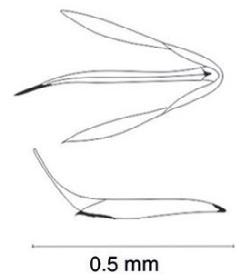

89
85
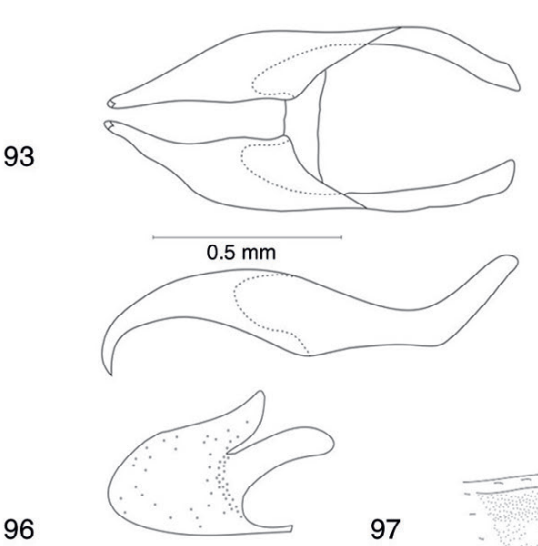

96

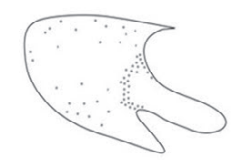

97

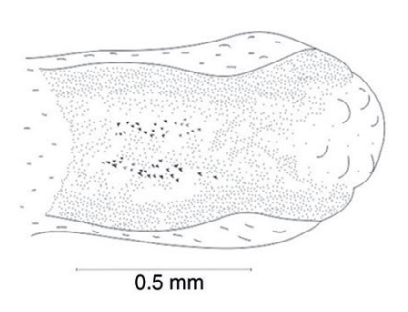

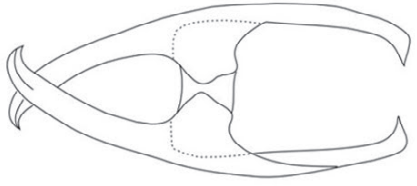

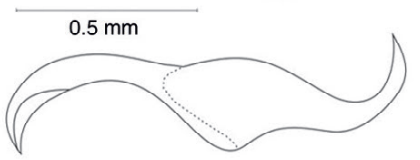

95

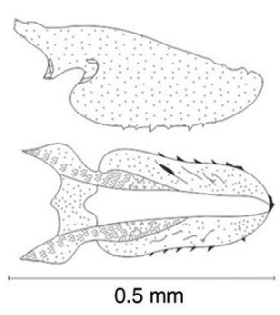

98

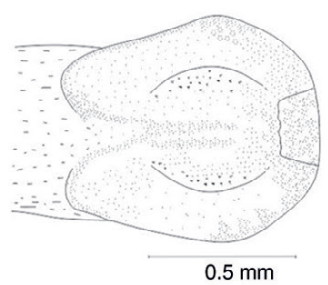

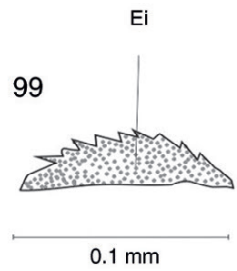

$0.25 \mathrm{~mm}$

Figs. 81-99.- Vista ventral del hipandrio ( ${ }^{(1)}$ ) de Subilla aliena (81), Xanthostigma corsicum* (83), Atlantoraphidia maculicollis* (84), Hispanoraphidia castellana* (87), Ohmella baetica* (88), Ohmella postulata* (90) y Ohmella casta* (92). Vista ventral y

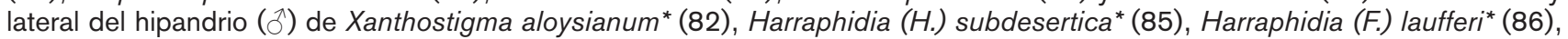
Ohmella bolivari* (89) y Ohmella libidinosa* (91). Vista dorsal y lateral de los parámeros (ð) de Phaeostigma (Ph.) notatum* (93) y de Phaeostigma (Ph.) italogallicum* (94). Vista dorsal y ventral de los parámeros ( $\left.{ }^{\Uparrow}\right)$ de Subilla aliena* (95). Vista dorsal de

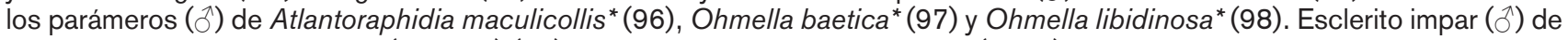
Venustoraphidia conviventibus (holotipo) (99). *Adaptadas de H. Aspöck et al. (1991).

Figs. 81-99.- Ventral view of hipandrium ( $\left({ }^{\star}\right)$ of Subilla aliena (81), Xanthostigma corsicum* (83), Atlantoraphidia maculicollis* (84), Hispanoraphidia castellana* (87), Ohmella baetica* (88), Ohmella postulata* (90) y Ohmella casta* (92). Ventral and lateral view of hipandrium ( $\left.\delta^{\Uparrow}\right)$ of Xanthostigma aloysianum* (82), Harraphidia (H.) subdesertica* (85), Harraphidia (F.) laufferi* (86), Ohmella bolivari* (89) y Ohmella libidinosa* (91). Dorsal and lateral view of paramera ( $\left.{ }^{\lambda}\right)$ of Phaeostigma $(P h$.) notatum*

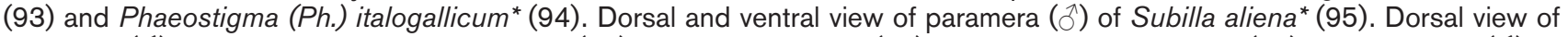
paramera ( $\left(^{\lambda}\right)$ of Atlantoraphidia maculicollis* (96), Ohmella baetica* (97) and Ohmella libidinosa* (98). Odd sclerite ( $\left.{ }^{\circledR}\right)$ of Venustoraphidia conviventibus (holotype) (99). *Adapted from H. Aspöck et al. (1991). 
100

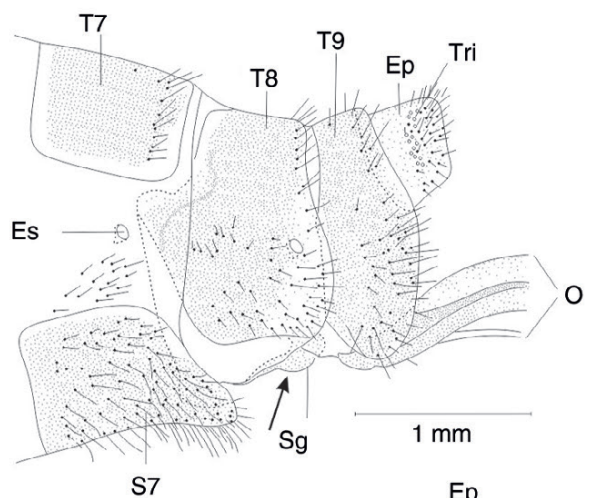

102

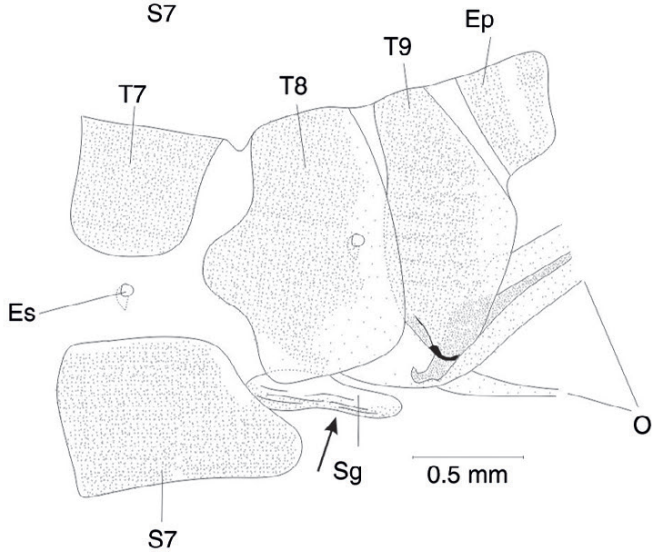

104

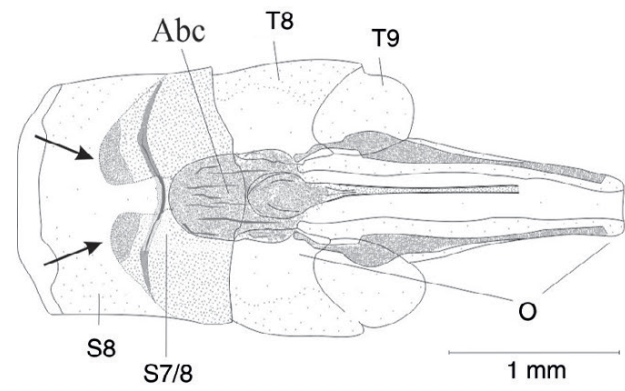

106

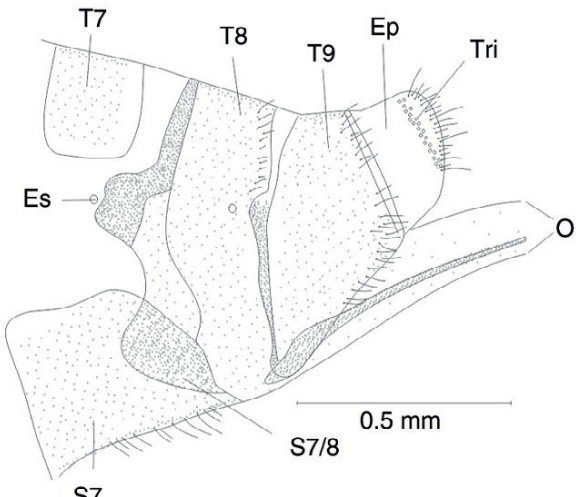

101

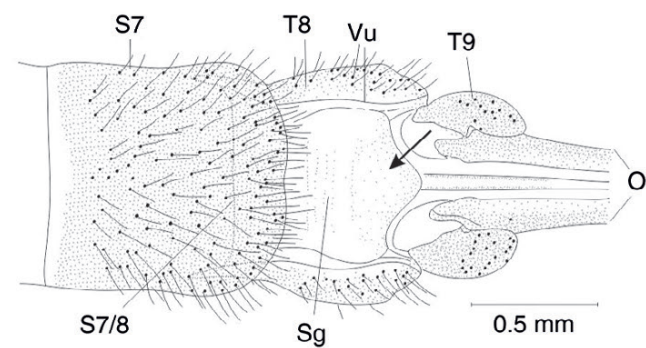

103

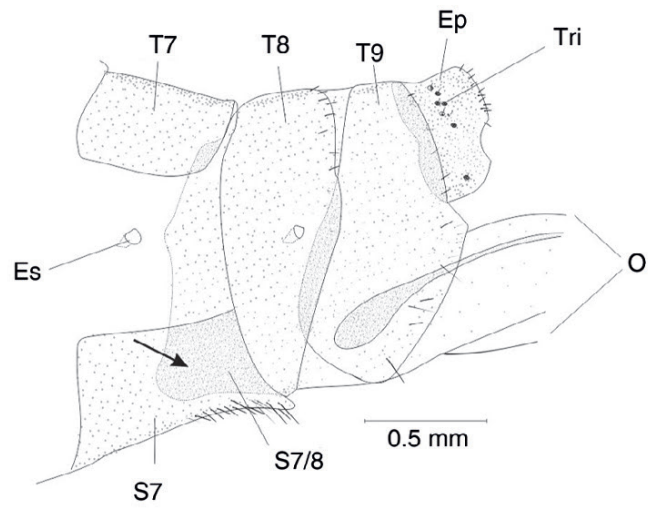

105

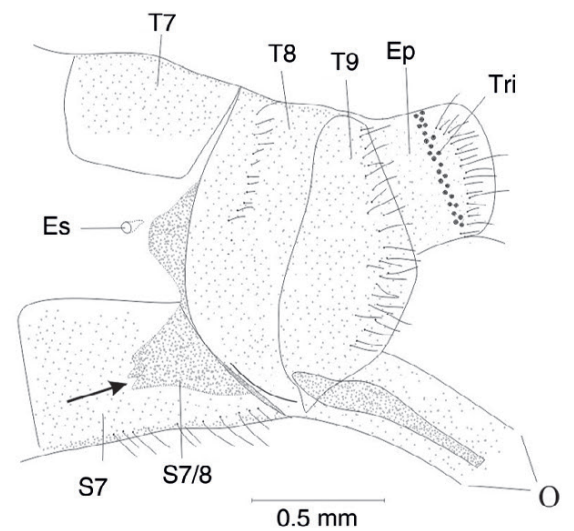

107

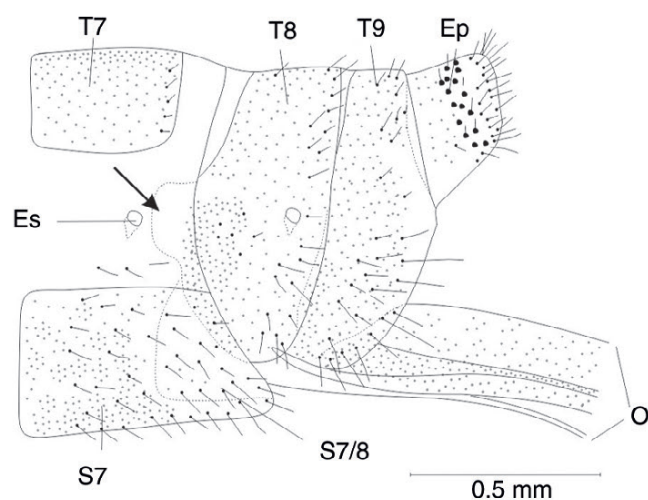

Figs. 100-107.- Genitalia (P) en vista lateral y ventral de Phaeostigma (Ph.) notatum* $(100,101)$ y de Subilla aliena (103, 104). Genitalia (+) en vista lateral de Phaeostigma (Ph.) italogallicum (102), Xanthostigma aloysianum (105), Xanthostigma corsicum (106) y Atlantoraphidia maculicollis* (107). Abc: atrio de la bolsa copuladora, Ep: ectoprocto, Es: espiráculo, S: esternito, Sg: subgenitalia, S 7/8: intersegmento 7/8, T: terguito, Tr: tricobotrios, O: ovopositor. ${ }^{*}$ Adaptadas de H. Aspöck et al. (1991).

Figs. 100-107.- Genitalia ( + ) in lateral and ventral view of Phaeostigma (Ph.) notatum* $(100,101)$ and Subilla aliena (103, 104). Genitalia (q) in lateral view of Phaeostigma (Ph.) italogallicum (102), Xanthostigma aloysianum (105), Xanthostigma corsicum (106) y Atlantoraphidia maculicollis* (107). Abc: bursa copulatrix atrium, Ep: ectoproct, Es: spiracle, S: sternite, Sg: subgenitalia, S 7/8: intersegment 7/8, T: tergite, Tr: trichobotria, O: ovopositor. * Adapted from H. Aspöck et al. (1991). 
108

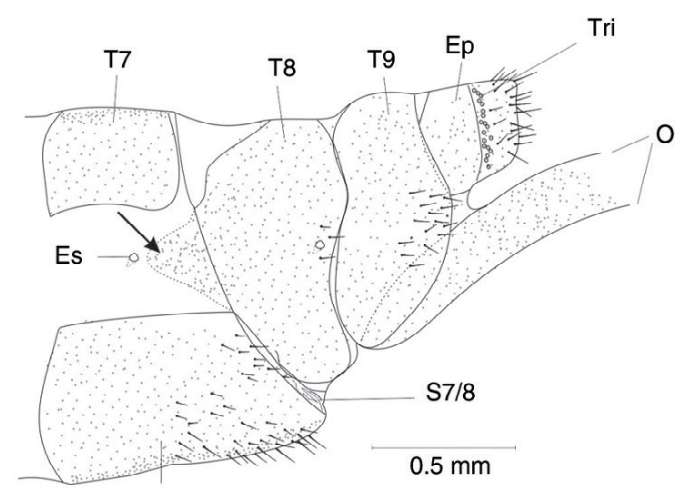

110

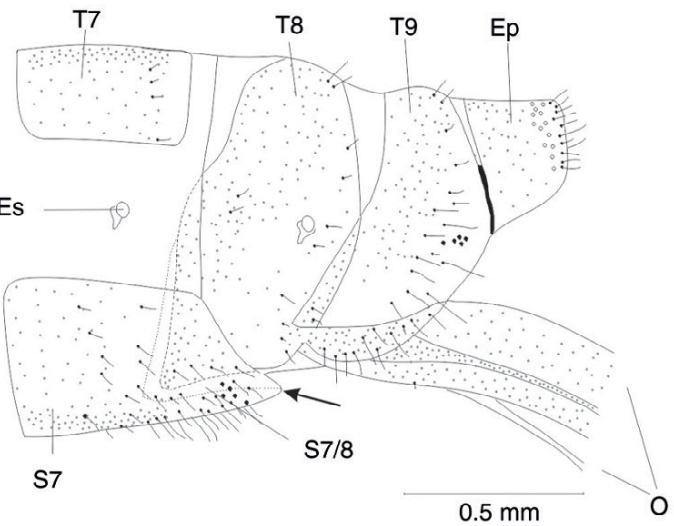

112

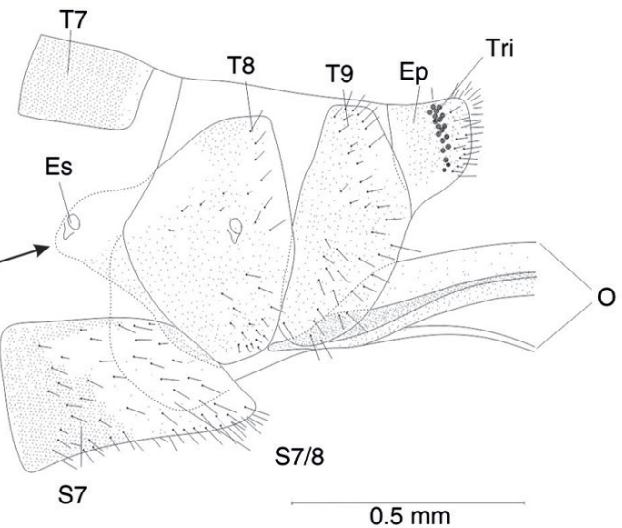

114

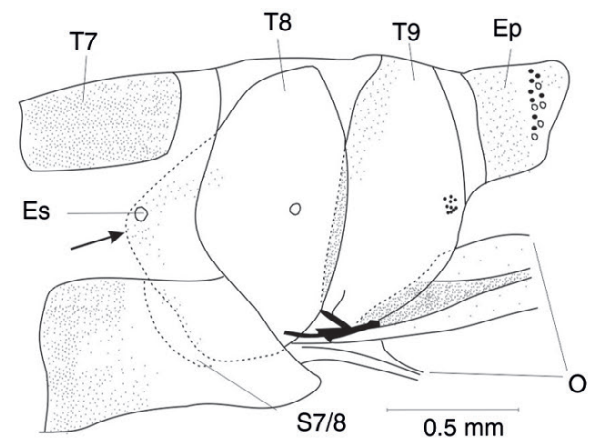

109

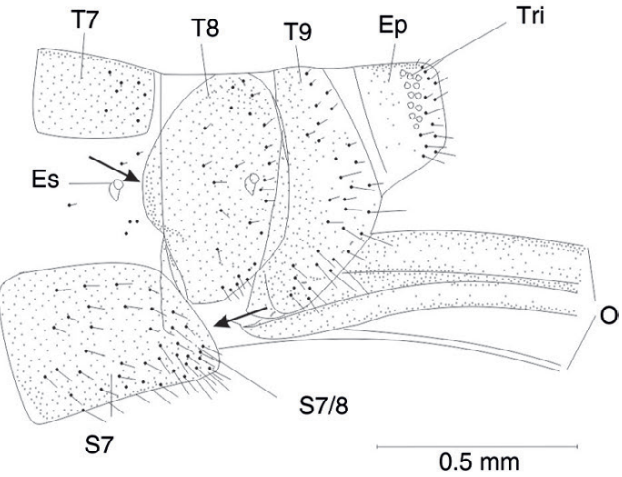

111

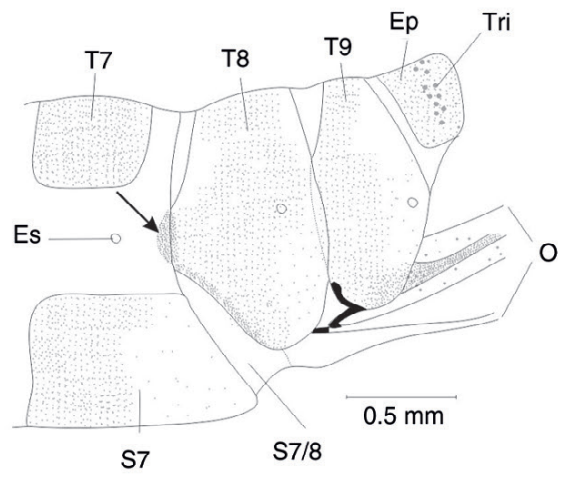

113

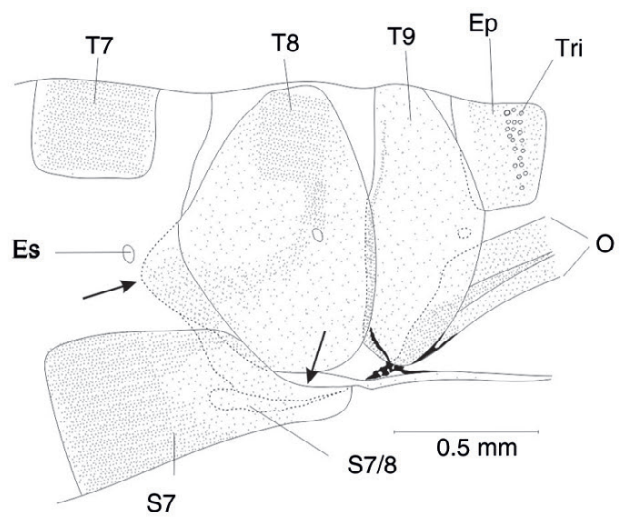

115

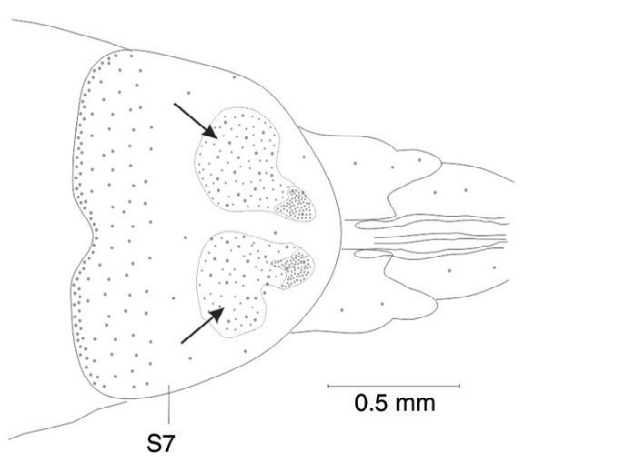

Figs. 108-115.- Genitalia ( $($ ) en vista lateral de Harraphidia (H.) subdesertica (108), Harraphidia (F.) laufferi* (109), Hispanoraphidia castellana* (110), Ohmella baetica* (111), Ohmella bolivari* (112), Ohmella postulata* (113) y Ohmella libidinosa* (114). Genitalia (q) en vista ventral de Ohmella libidinosa (115). Ep: ectoprocto, Es: espiráculo, S: esternito, S 7/8: intersegmento 7/8, T: terguito, Tr: tricobotrios, O: ovopositor. * Adaptadas de H. Aspöck et al. (1991).

Figs. 108-115.-Genitalia (+) in lateral view of Harraphidia (H.) subdesertica(108), Harraphidia(F.) laufferi*(109), Hispanoraphidia castellana* (110), Ohmella baetica* (111), Ohmella bolivari* (112), Ohmella postulata* (113) y Ohmella libidinosa* (114). Genitalia (P) in ventral view of Ohmella libidinosa (115). Ep: ectoproct, Es: spiracle, S: sternite, S 7/8: intersegment 7/8, T: tergite, Tr: trichobotria, O: ovopositor. ${ }^{*}$ Adapted from H. Aspöck et al. (1991). 
116

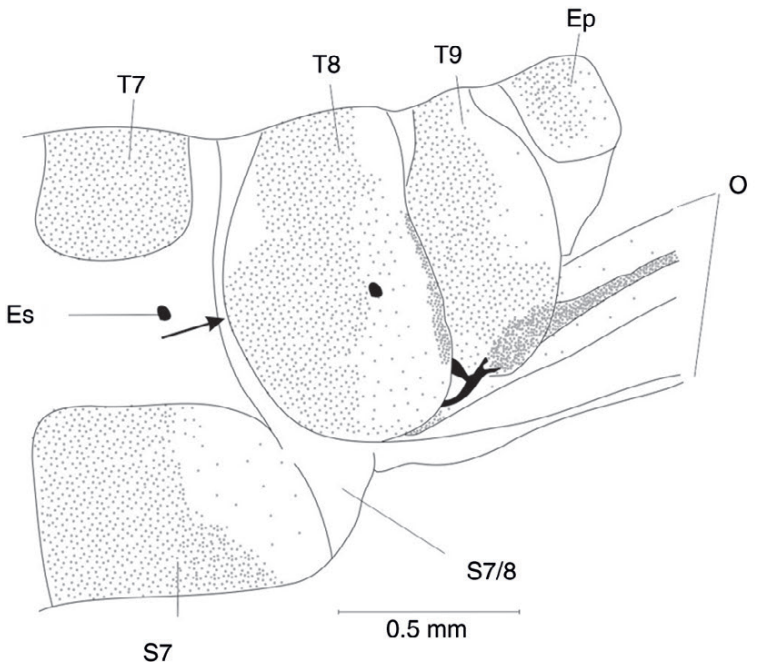

S7

118

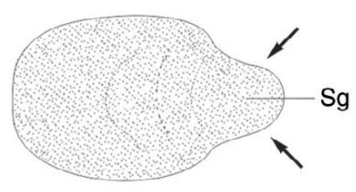

$0.5 \mathrm{~mm}$

122

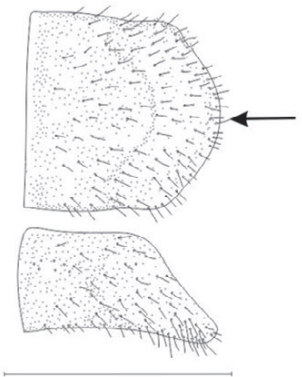

$0.5 \mathrm{~mm}$
119
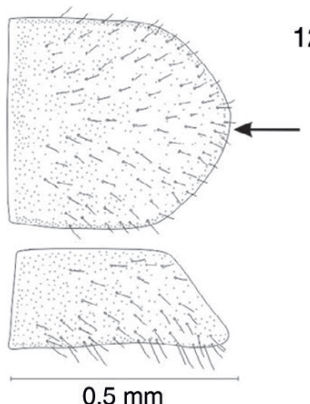

123
117

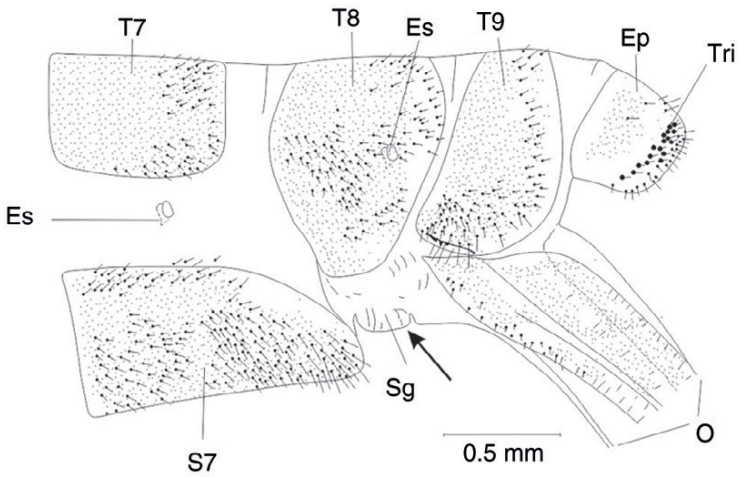

120

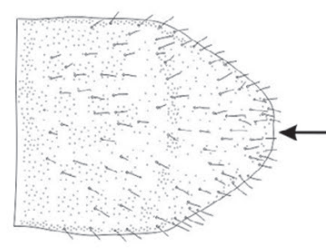

121

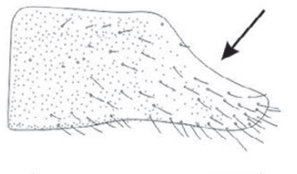

$0.5 \mathrm{~mm}$
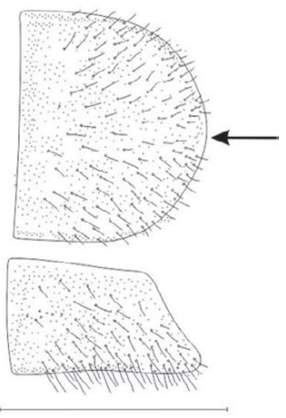

$0.5 \mathrm{~mm}$

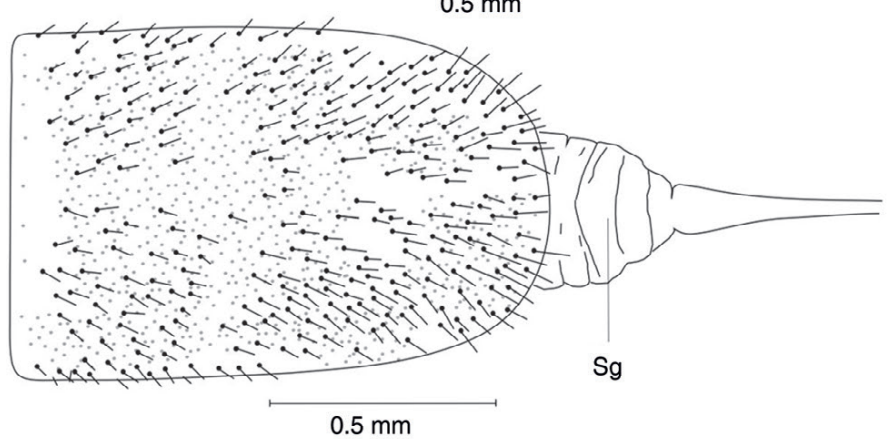

Figs. 116-123.- Genitalia () en vista lateral de Ohmella casta* (116) y Fibla (F.) hesperica* (117). Subgenitalia ( $($ ) en vista ventral de Phaeostigma (Ph.) italogallicum (118) y de Fibla (F.) hesperica* (123). $7^{\circ}$ esternito ( ) en vista ventral y lateral de Ohmella baetica* (119), Ohmella postulata* (120), Ohmella libidinosa* (121) y Ohmella casta* (122). Ep: ectoprocto, Es: espiráculo, S: esternito, Sg: subgenitalia, S 7/8: intersegmento 7/8, T: terguito, Tr: tricobotrios, O: ovopositor. ${ }^{*}$ Adaptadas de H. Aspöck et al. (1991).

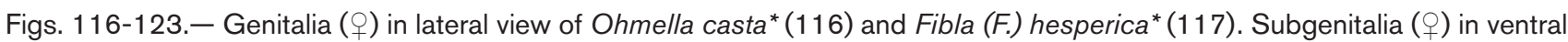
view of Phaeostigma (Ph.) italogallicum (118) and Fibla (F.) hesperica* (123). 7th sternite (P) in ventral and lateral view of 119) Ohmella baetica* (119), Ohmella postulata* (120), Ohmella libidinosa* (121) and Ohmella casta* (122). Ep: ectoproct, Es: spiracle, S: sternite, Sg: subgenitalia, S 7/8: intersegment 7/8, T: tergite, Tr: trichobotria, O: ovopositor. * Adapted from H. Aspöck et al. (1991). 

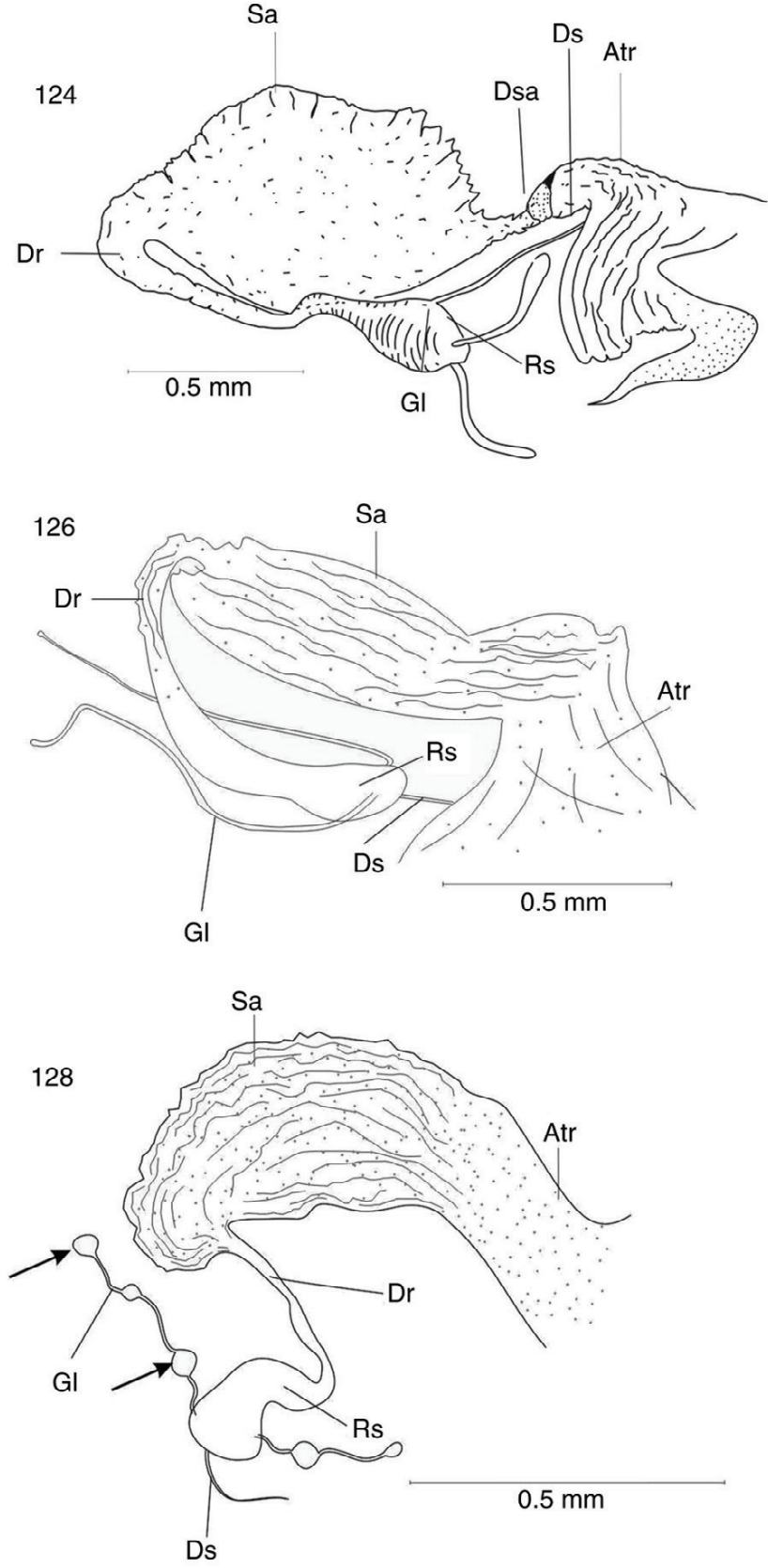

130
125
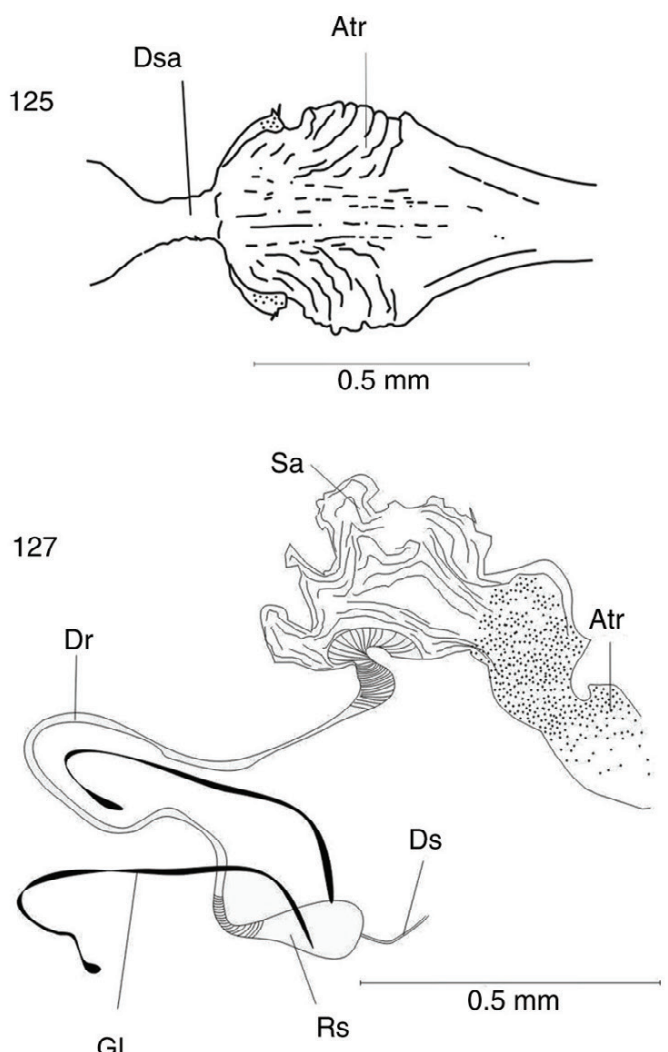

129

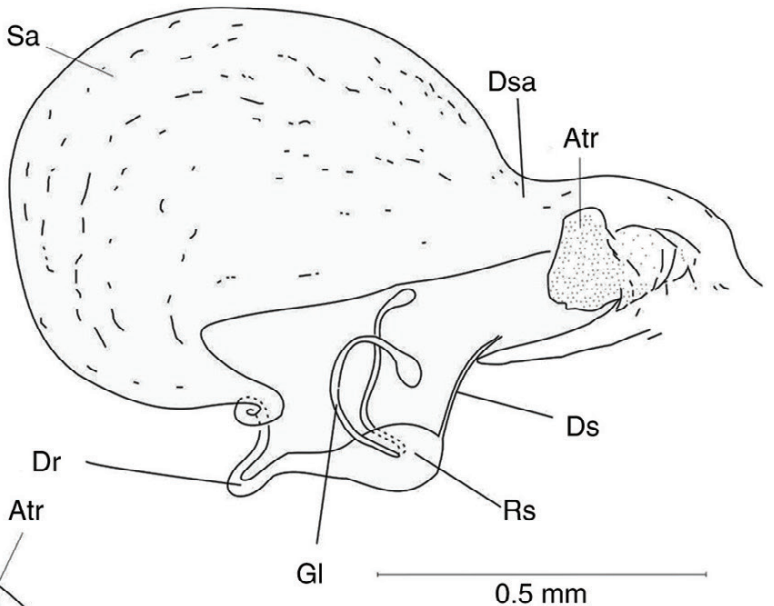

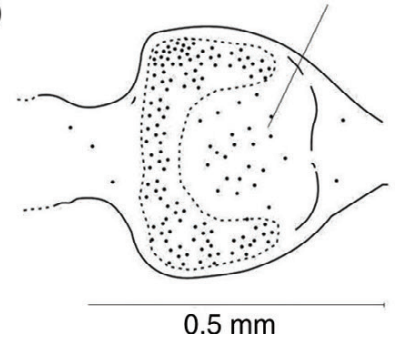

Figs. 124-130.- Genitalia interna ( $($ ) en vista lateral y atrio de la bolsa copuladora en vista dorsal de Phaeostigma (Ph.) notatum* $(124,125)$. Genitalia interna (+) en vista lateral de Subilla aliena (126), Xanthostigma aloysianum (127), Xanthostigma corsicum (128) y Atlantoraphidia maculicollis* (129). Atrio de la bolsa copuladora ( + ) en vista dorsal de Atlantoraphidia maculicollis $(130=)$. Atr: atrio de la bolsa copuladora, Bc: bolsa copuladora, Dr: ductus receptaculi, Ds: ductus seminis, Dsa: ductus saculi, Gl: glándulas accesorias, Rs: receptáculo seminal, Sa: sáculo de la bolsa copuladora. * Adaptadas de H. Aspöck et al. (1991).

Figs. 124-130.- Internal genitalia $(+)$ in lateral view and copulatrix bursa atrium in dorsal view of Phaeostigma (Ph.) notatum ${ }^{*}$ $(124,125)$. Internal genitalia (q) in lateral view of Subilla aliena (126), Xanthostigma aloysianum (127), Xanthostigma corsicum (128) and Atlantoraphidia maculicollis* (129). Copulatrix bursa atrium (ㅇ) in dorsal view of Atlantoraphidia maculicollis (130). Atr: copulatrix bursa atrium, Bc: copulatrix bursa, Dr: ductus receptaculi, Ds: ductus seminis, Dsa: ductus saculi, Gl: accesory glands, Rs: seminal receptacle, Sa: copulatrix bursa saculus. * Adapted from H. Aspöck et al. (1991). 
131

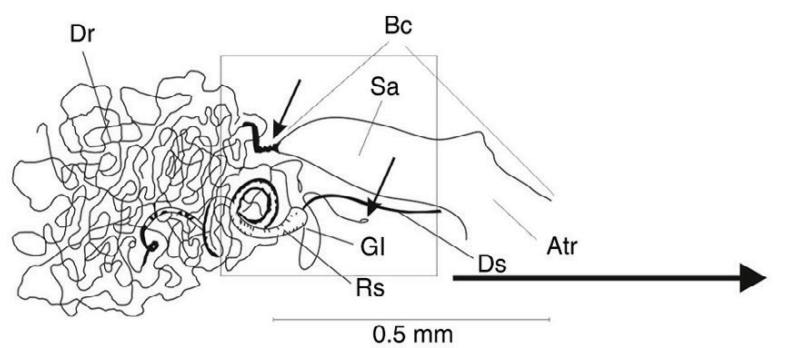

132

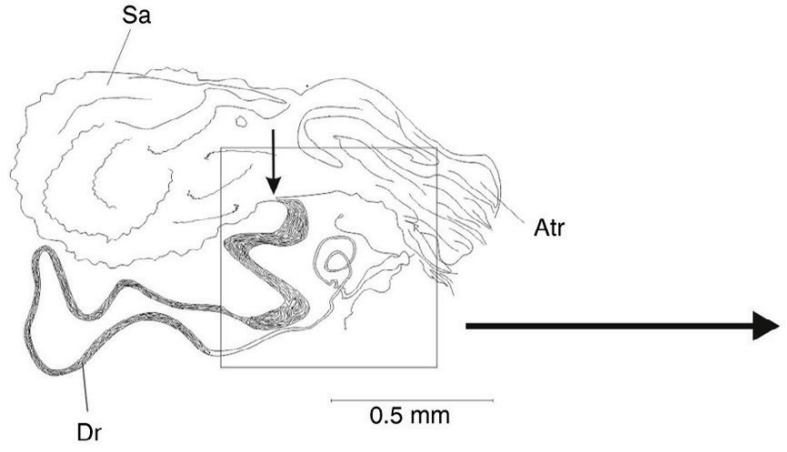

133

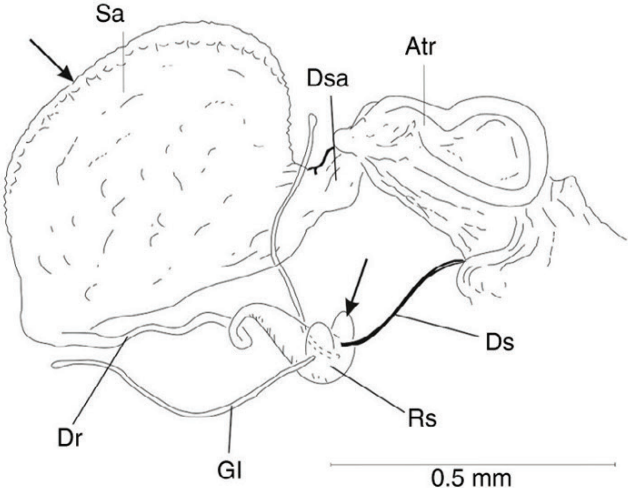

135

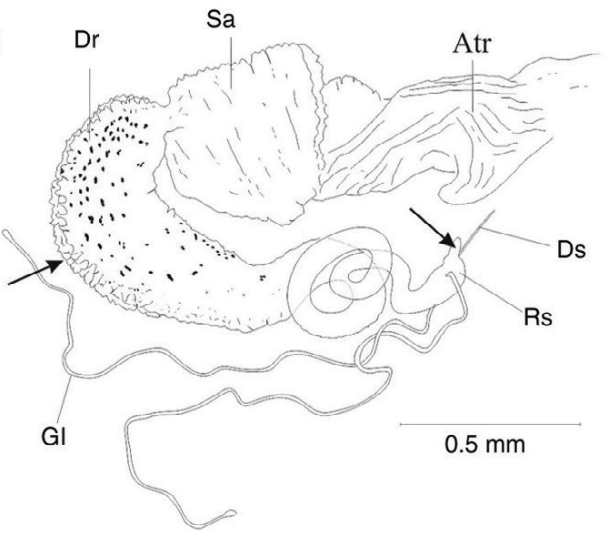

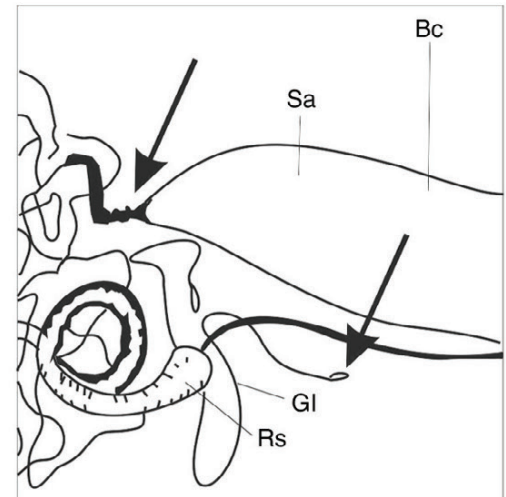

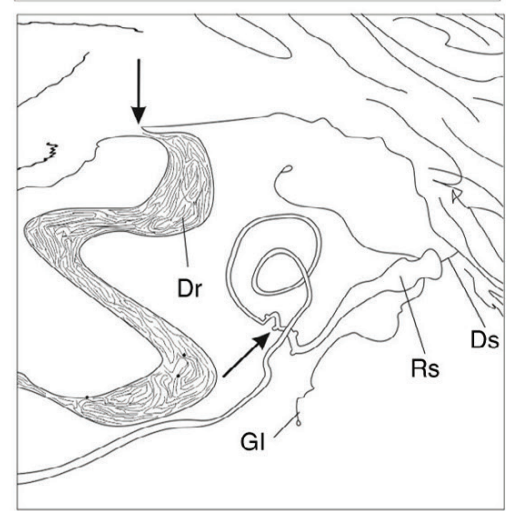

134
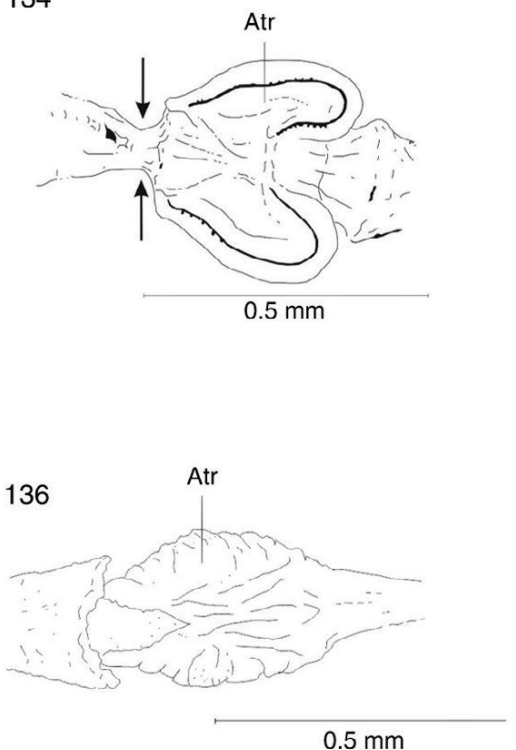

Figs. 131-136.- Genitalia interna $(Q)$ en vista lateral y ampliada la zona del ductus receptaculi y receptáculo seminal de Harraphidia (F.) laufferi* (131) y Harraphia (F.) subdesertica (132). Genitalia interna ( $($ ) en vista lateral y atrio de la bolsa copuladora en vista dorsal de Hispanoraphidia castellana* $(133,134)$ y Ohmella bolivari* $(135,136)$. Atr: atrio de la bolsa copuladora, Bc: bolsa copuladora, Dr: ductus receptaculi, Ds: ductus seminis, Gl: glándulas accesorias, Rs: receptáculo seminal, Sa: sáculo de la bolsa copuladora. * Adaptadas de H. Aspöck et al. (1991).

Figs. 131-136. - Internal genitalia $(+)$ in lateral view, magnified ductus receptaculi area and seminal receptacle of $H a r r a p h i d i a(F$. laufferi* (131) and Harraphia (F.) subdesertica (132). Internal genitalia ( $($ ) in lateral view and copulatrix bursa atrium in dorsal view of Hispanoraphidia castellana* $(133,134)$ and Ohmella bolivari* $(135,136)$. Atr: copulatrix bursa atrium, Bc: copulatrix bursa, Dr: ductus receptaculi, Ds: ductus seminis, Gl: accesory glands, Rs: seminal receptacle, Sa: copulatrix bursa saculus. * Adapted from H. Aspöck et al. (1991). 
137

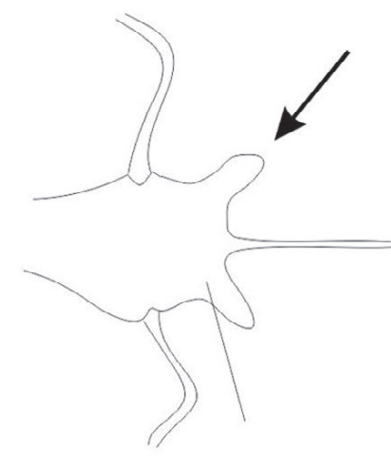

Rs
138

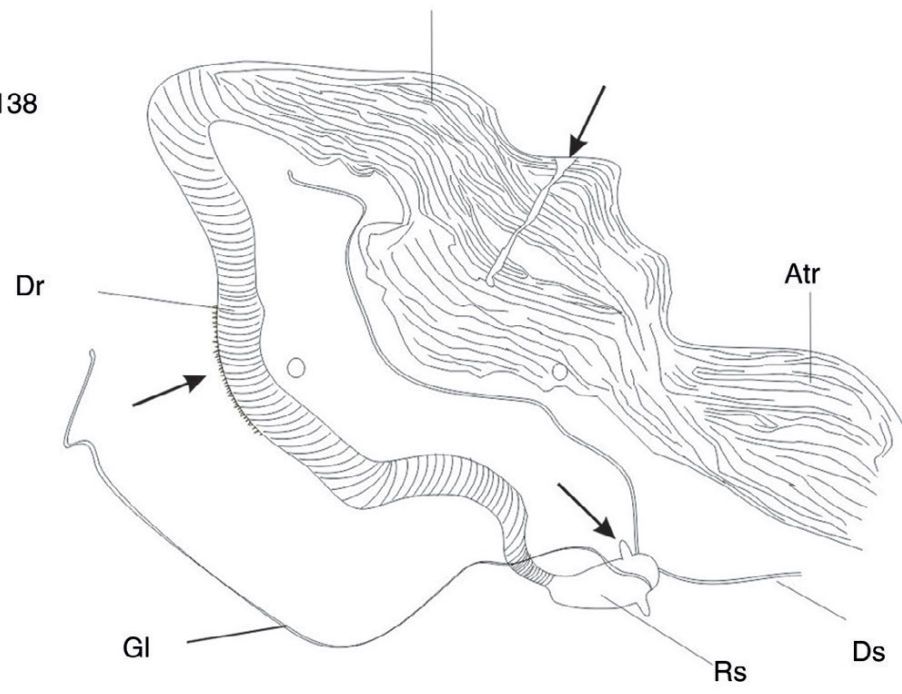

140
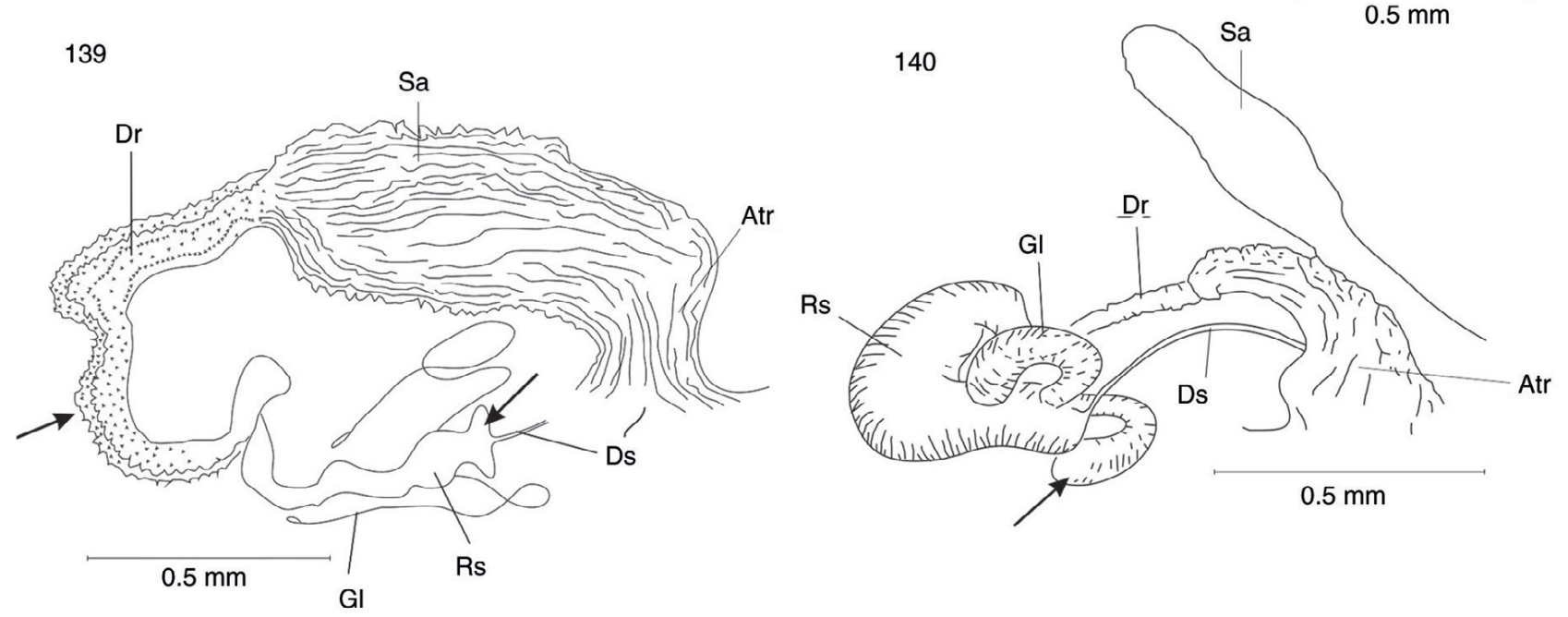

Figs. 137-140.- Detalle del receptáculo seminal en vista dorsal de Ohmella bolivari* (137). Genitalia interna ()) en vista lateral de Ohmella postulata (138), Ohmella libidinosa (139) y Fibla (F.) hesperica* (140). Atr: atrio de la bolsa copuladora, Dr: ductus receptaculi, Ds: ductus seminis, Dsa: ductus saculi, Gl: glándulas accesorias, Rs: receptáculo seminal, Sa: sáculo de la bolsa copuladora. * Adaptadas de H. Aspöck et al. (1991).

Figs. 137-140.- Particular of seminal receptacle in dorsal view of Ohmella bolivari* (137). Internal genitalia ( + ) in lateral view of Ohmella postulata (138), Ohmella libidinosa (139) and Fibla (F.) hesperica* (140). Atr: copulatrix bursa atrium, Dr: ductus receptaculi, Ds: ductus seminis, Dsa: ductus saculi, Gl: accesory glands, Rs: seminal receptacle, Sa: copulatrix bursa saculus. * Adapted from H. Aspöck et al. (1991). 
141

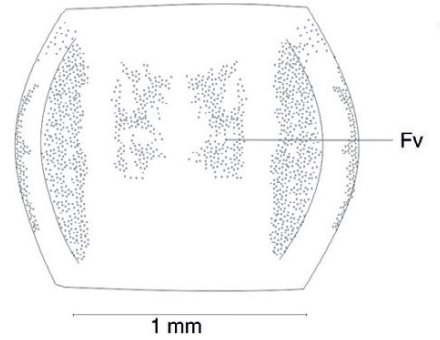

142

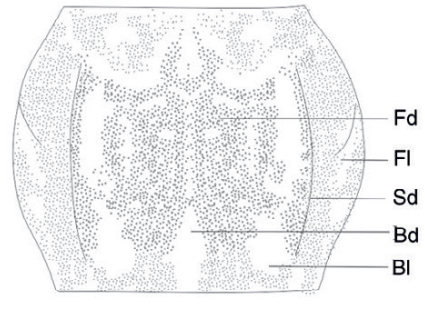

143

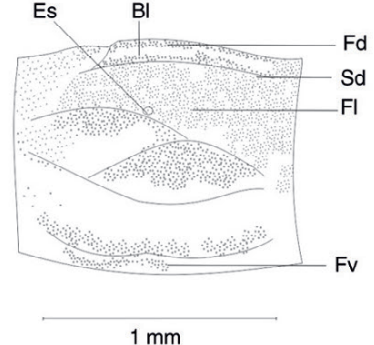

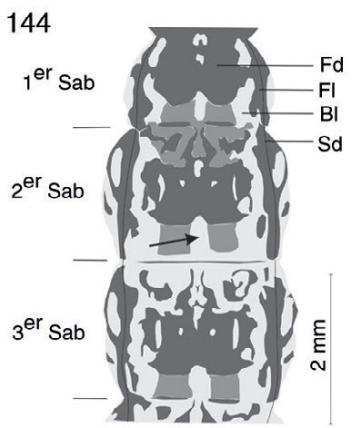

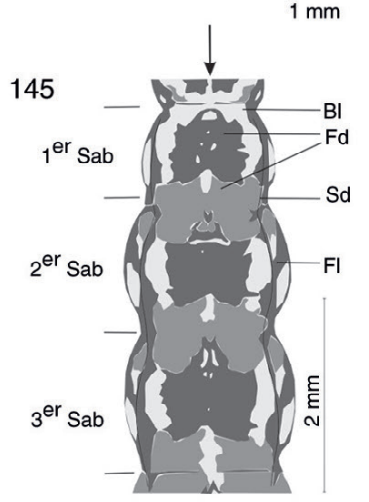

146

147

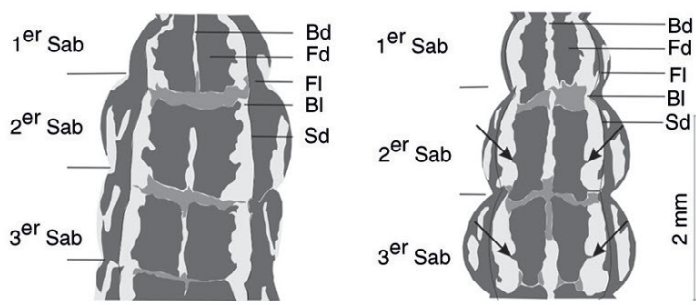

148

149
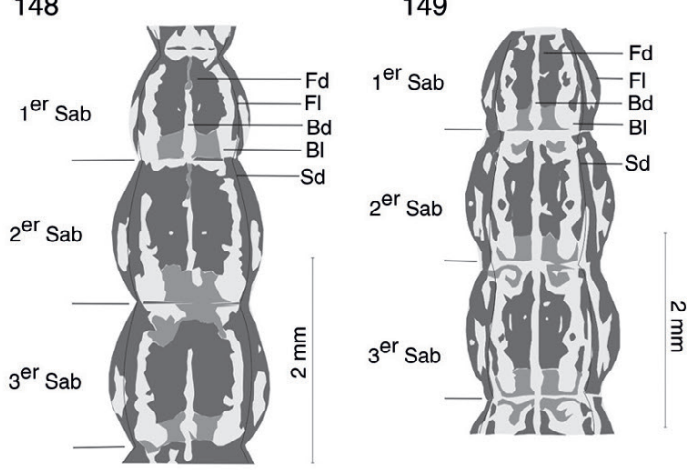

150

151

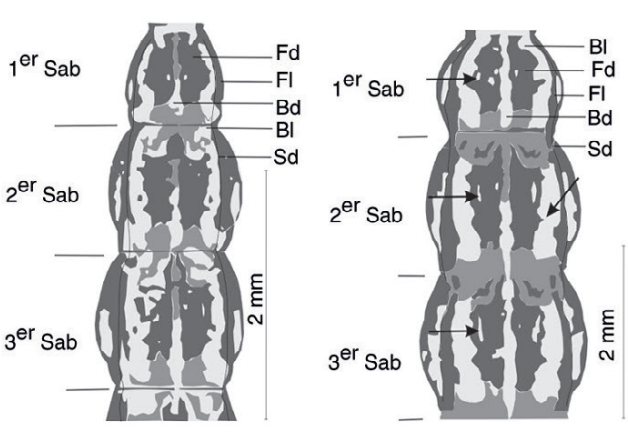

154

155

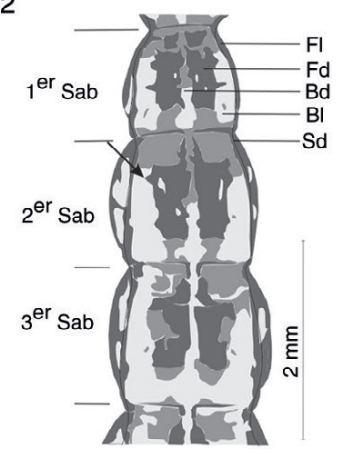

153
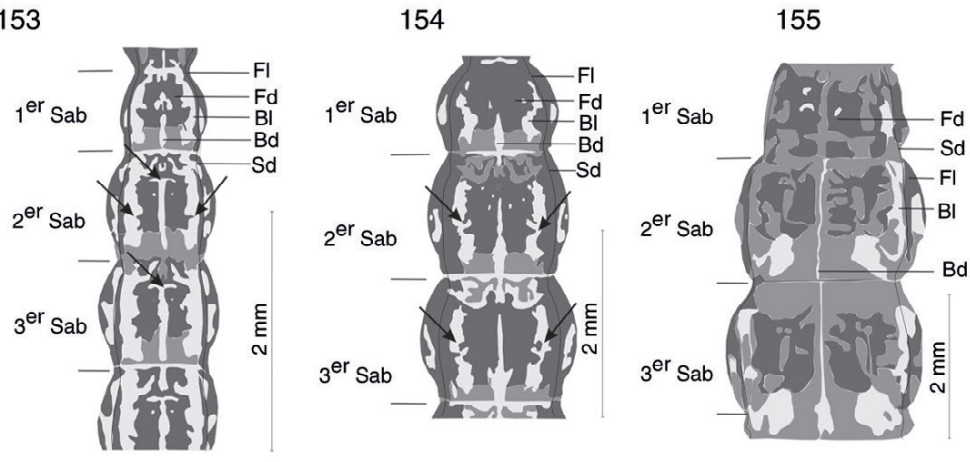

Figs. 141-155.- Tercer segmento abdominal de la larva de Ohmella casta en vista dorsal ${ }^{\star}(141)$, ventral ${ }^{*}(142)$ y lateral ${ }^{\star}(143)$. Vista dorsal de los tres primeros segmentos del abdomen de las larvas de Phaeostigma (Ph.) notatum* (144), Subilla aliena* (145), Xanthostigma aloysianum ** (146), Xanthostigma corsicum* (147), Atlantoraphidia maculicollis* (148), Harraphidia (F.) laufferi* (149), Hispanoraphidia castellana*(150), Ohmella baetica*(151), Ohmella postulata*(152), Ohmella libidinosa*(153),

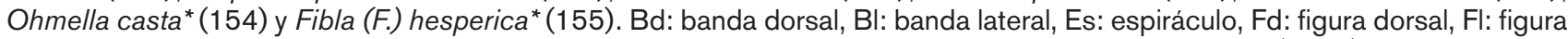
lateral, Fv: figura ventral, Sab: segmento abdominal, Sd: sutura dorsal. * Adaptadas de H. Aspöck et al. (1991), ${ }^{* *}$ : adaptada de una fotografía de R.A. Pantaleoni.

Figs. 141-155.- 3rd abdominal segment in the larva of Ohmella casta: dorsal* $(141)$, ventral* (142) and lateral view* (143). Dorsal view of three first abdominal segments in the larvae of Phaeostigma (Ph.) notatum* (144), Subilla aliena* (145), Xanthostigma aloysianum** (146), Xanthostigma corsicum* (147), Atlantoraphidia maculicollis* (148), Harraphidia (F.) laufferi* (149), Hispanoraphidia castellana*(150), Ohmella baetica*(151), Ohmella postulata*(152), Ohmella libidinosa*(153), Ohmella casta* $^{*}(154)$ and Fibla (F.) hesperica* (155). Bd: dorsal strip, Bl: lateral strip, Es: spiracle, Fd: dorsal figure, Fl: lateral figure, Fv: ventral figure, Sab: abdominal segment, Sd: dorsal suture. * Adapted from H. Aspöck et al. (1991), ${ }^{* *}$ : adapted from a photo by R.A. Pantaleoni. 
156

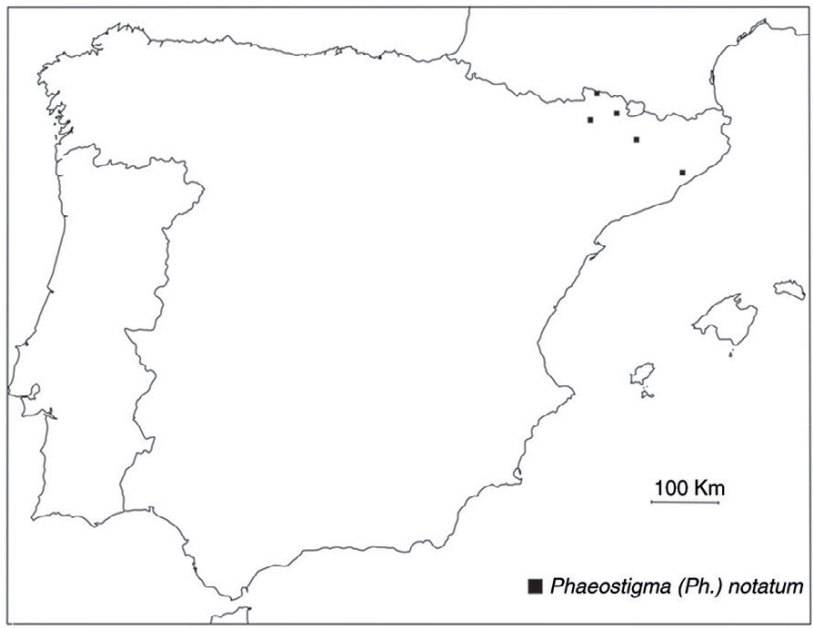

158

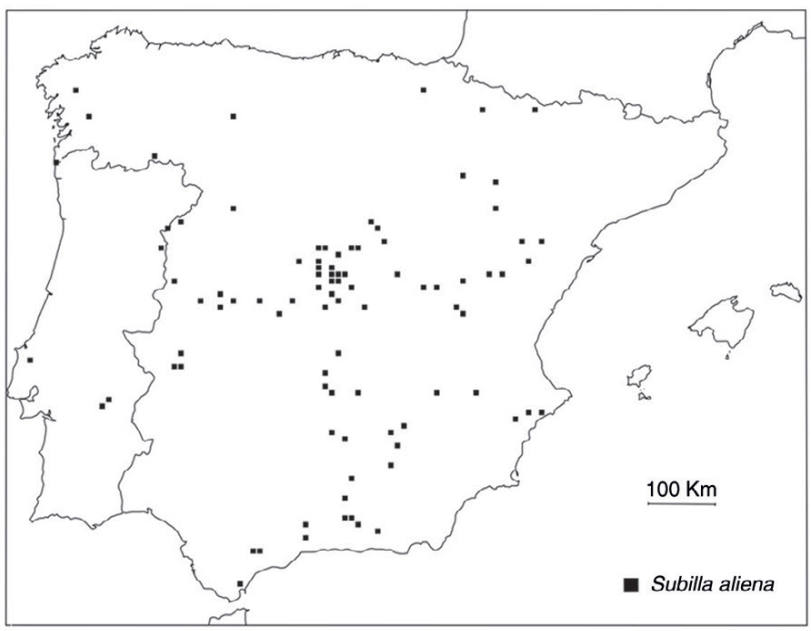

160

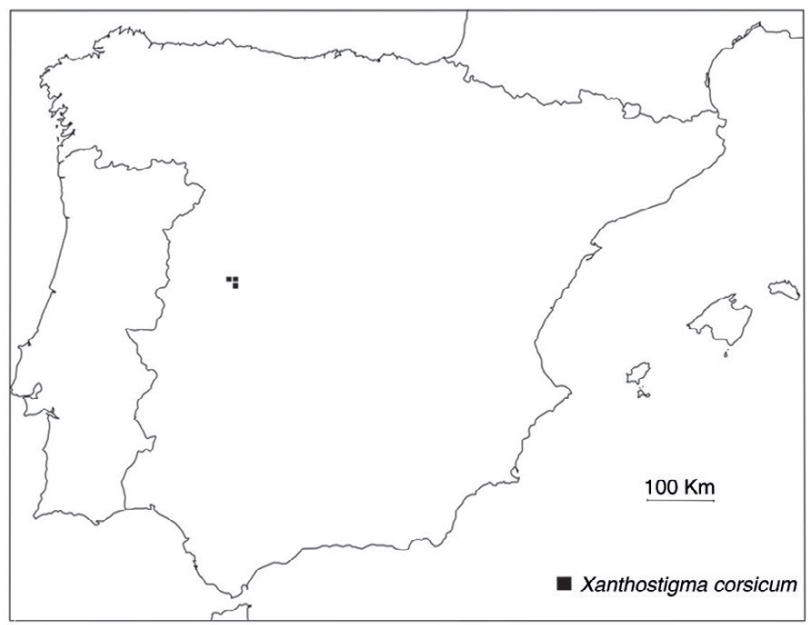

157

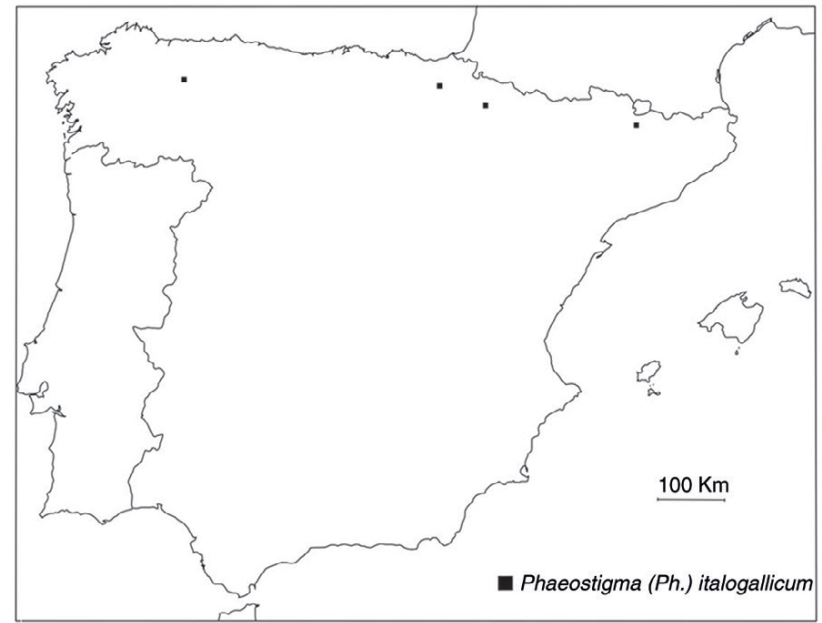

159

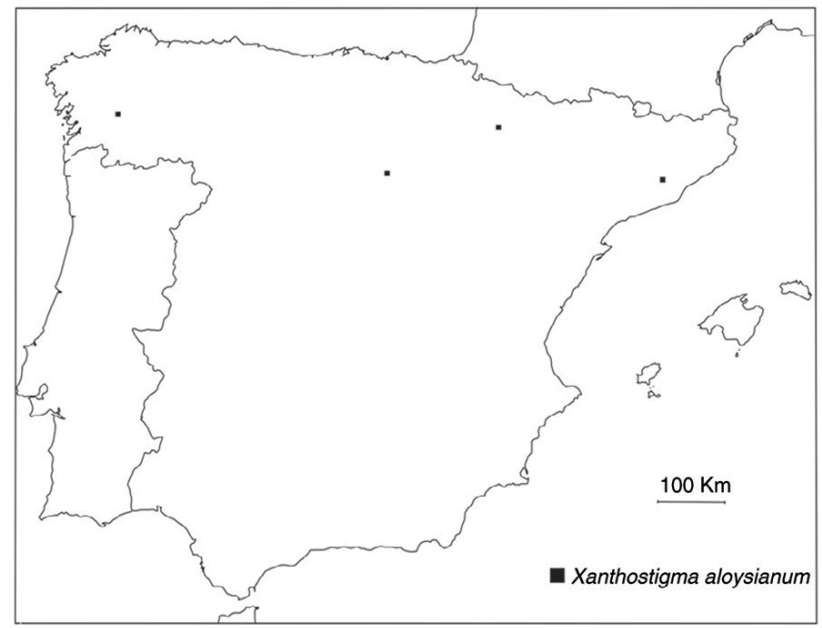

161

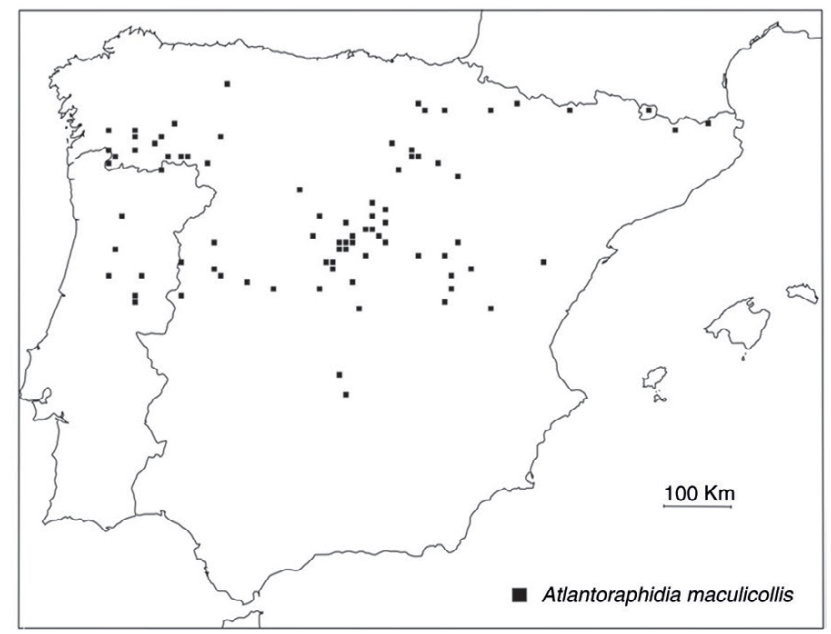

Figs. 156-161. - Mapas de la distribución en la Península lbérica de Phaeostigma (Ph.) notatum (156), Phaeostigma (Ph.) italogallicum (157), Subilla aliena (158), Xanthostigma aloysianum (159), Xanthostigma corsicum (160) y Atlantoraphidia maculicollis (161).

Figs. 156-161.- Distribution maps in the Iberian Peninsula of Phaeostigma (Ph.) notatum (156), Phaeostigma (Ph.) italogallicum (157), Subilla aliena (158), Xanthostigma aloysianum (159), Xanthostigma corsicum (160) and Atlantoraphidia maculicollis (161). 
162

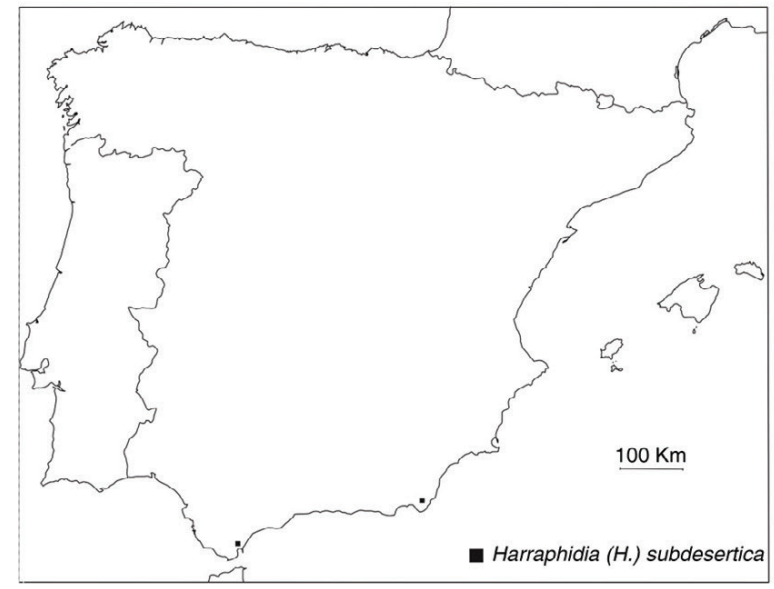

164

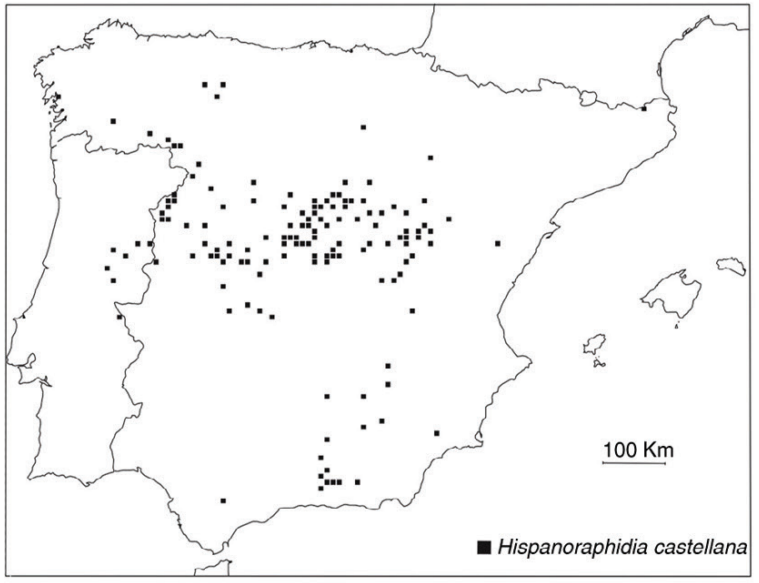

166

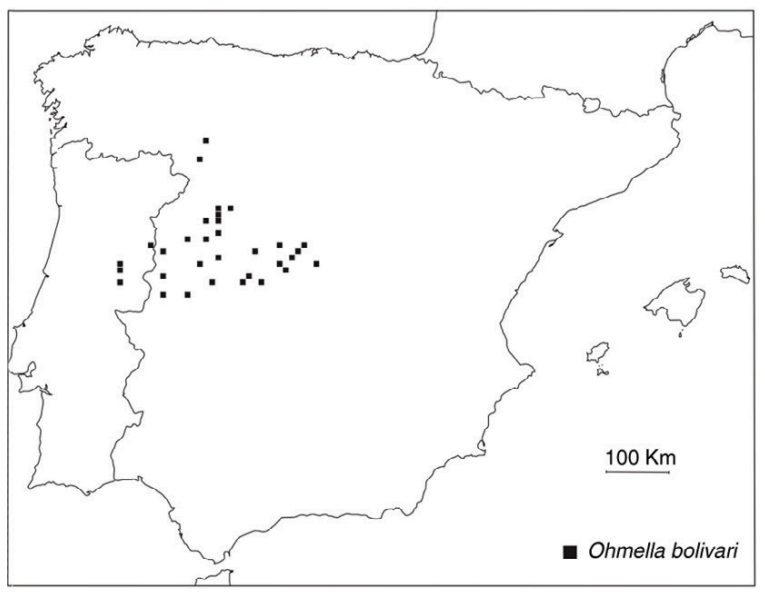

163

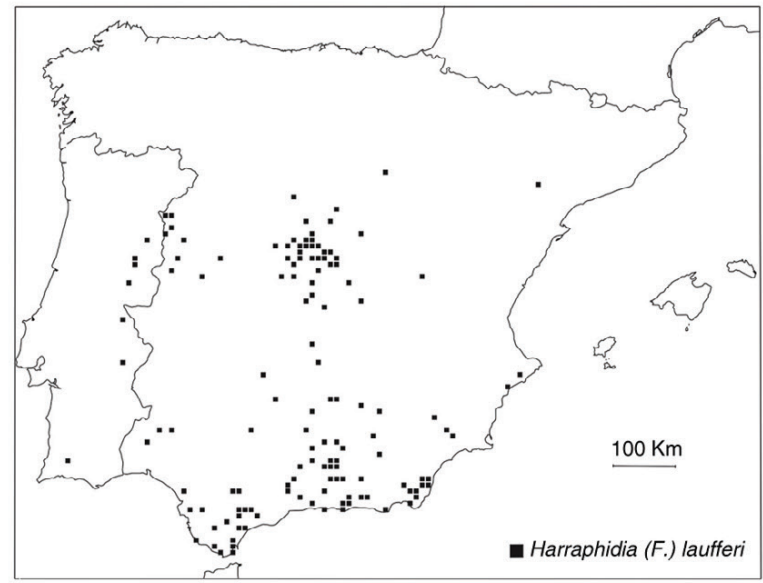

165

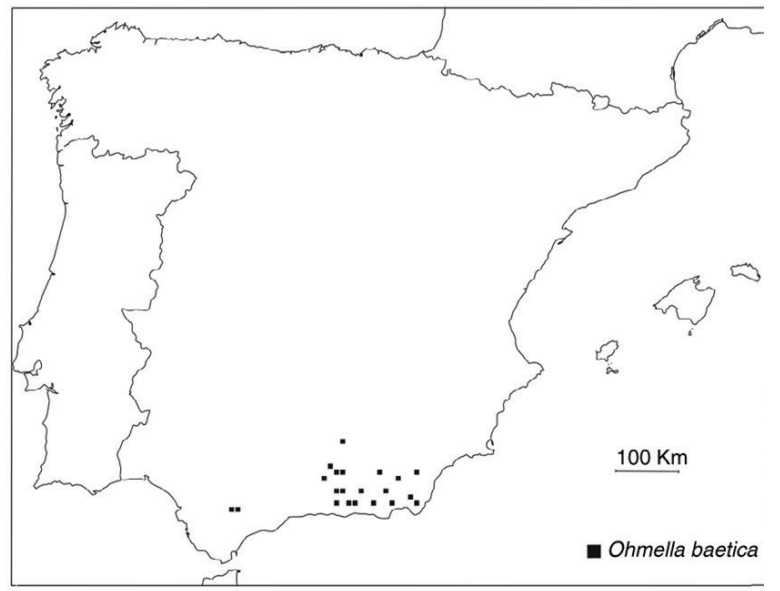

167

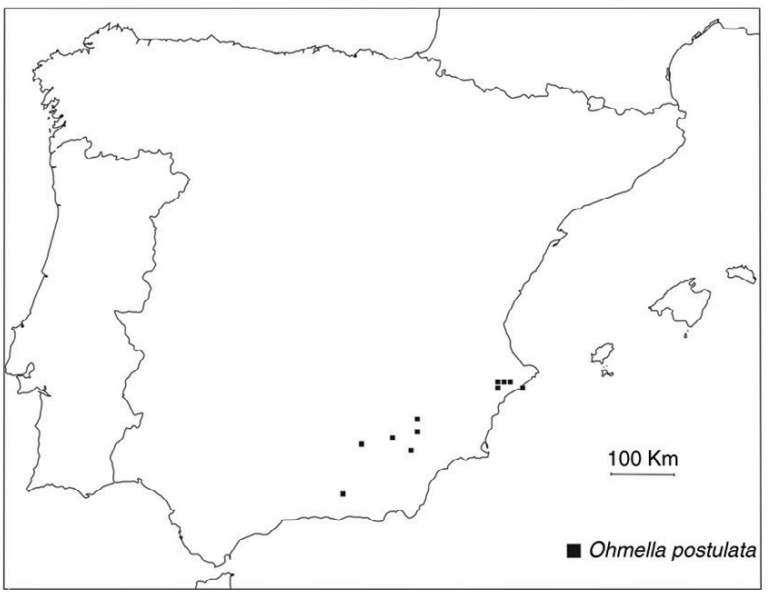

Figs. 162-167.- Mapas de la distribución en la Península lbérica de Harraphidia (H.) subdesertica (162), Harraphidia (F.) laufferi (163), Hispanoraphidia castellana (164), Ohmella baetica (165), Ohmella bolivari (166) y Ohmella postulata (167).

Figs. 162-167.- Distribution maps in the Iberian Peninsula of Harraphidia (H.) subdesertica (162), Harraphidia (F.) laufferi (163), Hispanoraphidia castellana (164), Ohmella baetica (165), Ohmella bolivari (166) and Ohmella postulata (167). 
168

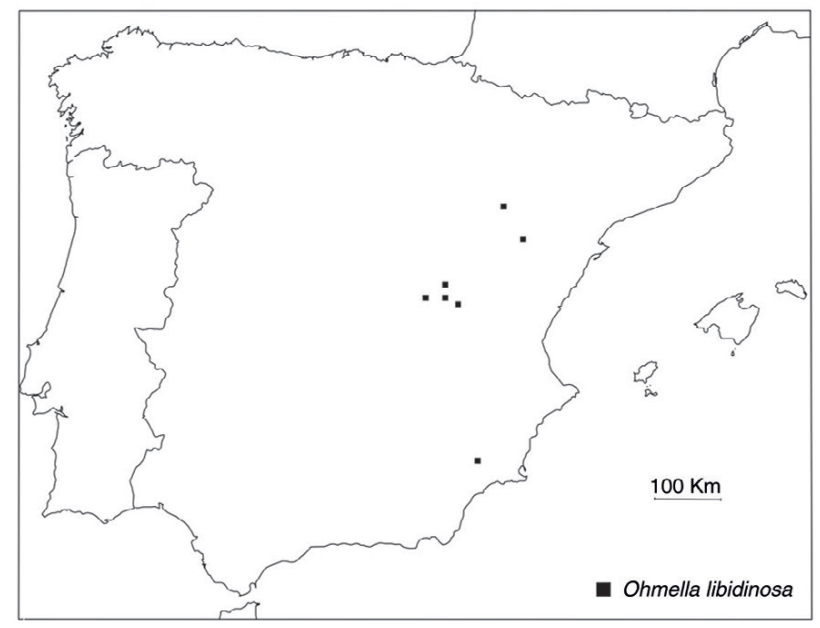

170

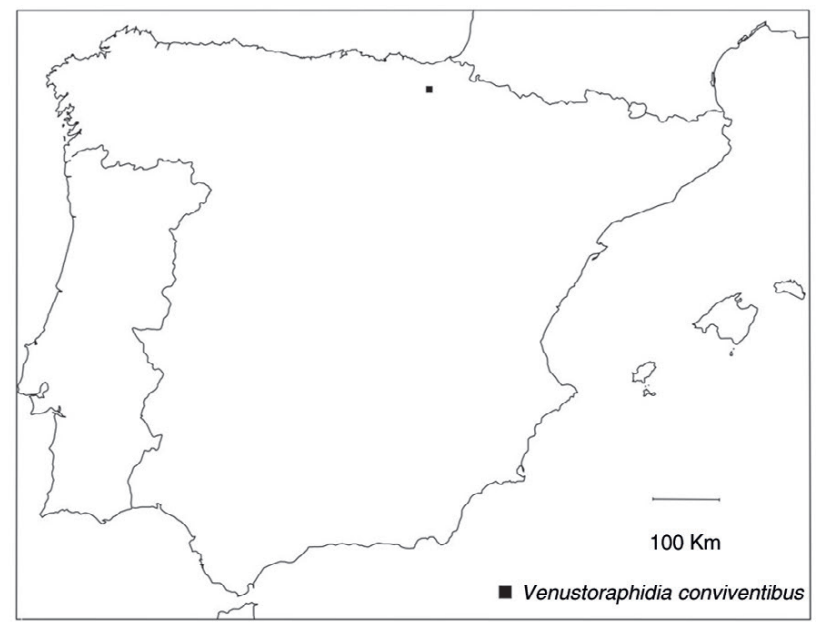

169

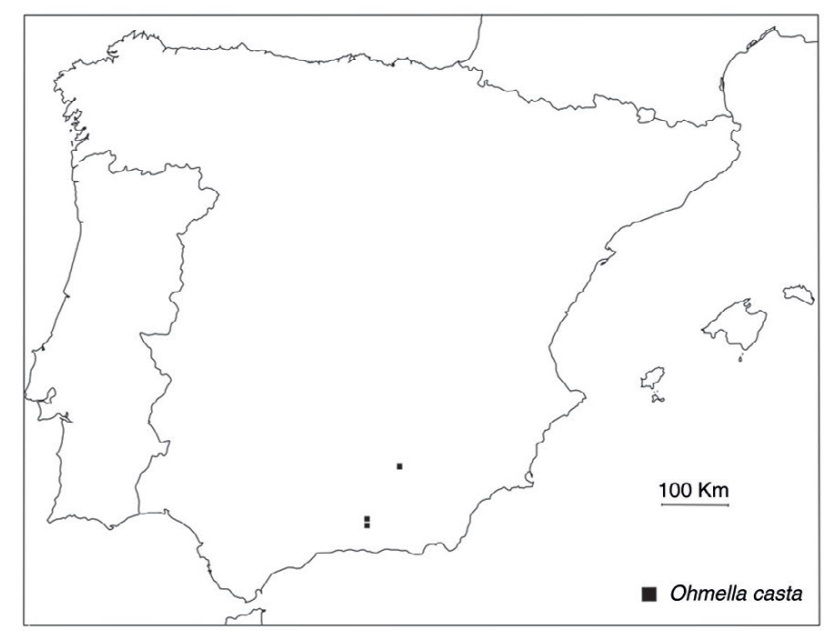

171

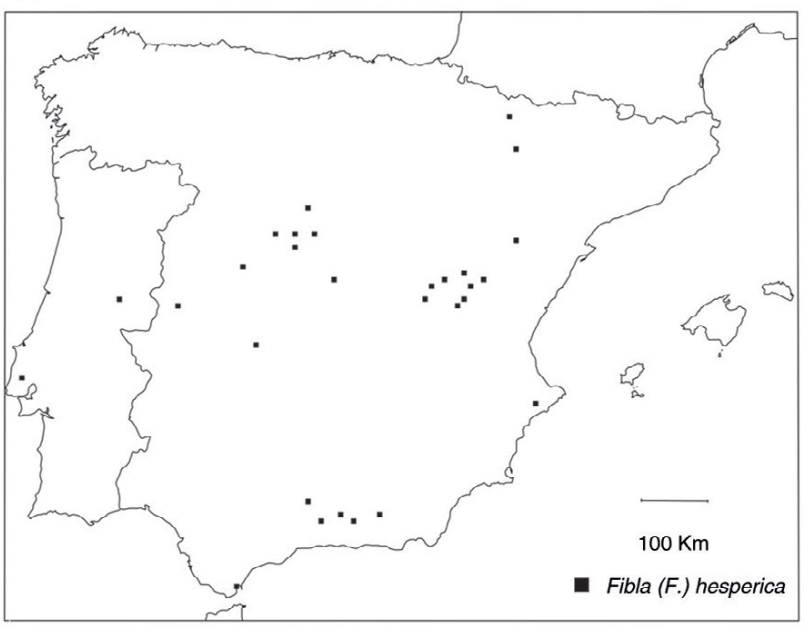

Figs. 168-171.- Mapas de la distribución en la Península lbérica de Ohmella libidinosa (168), Ohmella casta (169), Venustoraphidia conviventibus (170) y Fibla (F.) hesperica (171).

Figs. 168-171.- Distribution maps in the Iberian Peninsula of Ohmella libidinosa (168), Ohmella casta (169), Venustoraphidia conviventibus (170) and Fibla (F.) hesperica (171). 


\section{Referencias}

Ábrahám, L., 1991. On the Neuropteroidea and Mecoptera of Baranya County, Hungary. A Janus Pannonius Múzeum Evkönyve, 35: 13-18.

Ábrahám, L., 2001. Somogy megye recésszárnyú-alkatúinak katalógusa (Megaloptera, Raphidioptera, Neuroptera). Natura Somogyiensis, 1: 253-260.

Achtelig, M., 1967. Über die Anatomie des Kopfes von Raphidia flavipes Stein und die Verwandschaftsbeziehungen der Raphidiidae zu den Megaloptera. Zoologische Jahrbücher. Abteilung für Anatomie und Ontogenie der Tiere, 84: 249-312.

Achtelig, M., 1974. Entwicklung von Gregarina raphidiae n. sp. (Sporozoa, Eugregarinida) aus dem Darm der Larven der Gattung Raphidia (Insecta, Raphidioptera). Archiv für Protistenkunde, 166: 80-98.

Achtelig, M., 1975a. Zur Feinstruktur und Funktion der Sporodukte von Gregarina raphidiae Achtelig (Sporozoa, Eugregarinida). Archiv für Protistenkunde, 117: 54-59.

Achtelig, M., 1975b. Die Abdomenbasis der Neuropteroidea (Insecta, Holometabola). Eine vergleichende anatomische Untersuchung des Skeletts und der Muskulatur. Zoomorphologie, 82: 201-242.

Achtelig, M., 1976. Indizien zur Monophylie der Raphidioptera und Megaloptera (Insecta, Holometabola). Verhandlungen der Deutschen Zoologischen Gesellschaft, 1976: 233.

Achtelig, M., 1977. Skelet und Musculatur des Abdomens weiblicher Raphidioptera (Insecta, Neuropteroidea). Zoologische Jahrbücher. Abteilung für Anatomie und Ontogenie der Tiere, 98: 137-164.

Achtelig, M., 1978. Entwicklung und Morphologie der inneren und äusseren weiblichen genitalorgane der Kamelhalsfliegen (Neuropteroidea: Raphidioptera). Entomologica Germanica, 4: 140-163.

Achtelig, M., 1997. Kamelhalsfliegen (Ins. Raphidioptera) aus der Umgebung von Würzburg. Galathea, 3: 48-51.

Achtelig, M. \& Kristensen, N. P., 1973. A re-examination of the relationships of the Raphidioptera (Insecta). Zeitschrift für Zoologische Systematik und Evolutionsforschung, 11: 268-274.

Acker, T. S., 1960. The comparative morphology of the male terminalia of Neuroptera (Insecta). Microentomology, 24: 25-84.

Acker, T. S., 1966. Courtship and Mating Behavior in Agulla Species (Neuroptera: Raphidiidae). Annals of the Entomological Society of America, 59: 1-6. http://dx. doi.org/10.1093/aesa/59.1.1

Adams, P. A., 1969. A new genus and species of Osmylidae (Neuroptera) from Chile and Argentina, with a discussion of planipennian genitalic homoligies. Postilla, 141: 1-11.

Aistleitner, U. \& Gruppe. A., 2004. Vorläufige Checkliste der Netzflügler (Insecta: Neuropteroidea) Vorarlbergs/ Austria occ. Vorarlberger Naturschau, 14: 153-160.

Albarda, H., 1889. Catalogue raisonné et synonymique des Névroptères observés dans les Pays-Bas et dans les Pays limitrophes. Tijdschrift voor Entomologie, 32: 211-376.
Albarda, H., 1891. Révision des Rhaphides. Tijdschrift voor Entomologie, 34: 65-119.

Albertina, I., Kathirithamby, J. \& Letardi, J., 1995. Neuropteroidea (Megaloptera, Raphidioptera, Planipennia), Mecoptera, Siphonaptera, Strepsiptera. Checklist delle specie della fauna italiana, 62: 1-20.

Alfken, J. D., 1939. Systematisches Verzeichnis der Netzflügler (Mecoptera, Megaloptera und Neuroptera) von Nordwestdeutschland. Abhandlungen herausgegeben vom Naturwissenschaftliche Verein zu Bremen, 31: 515-520.

Alrouechdi, K., 1982. Bio-écologie de Chrysoperla carnea (Stephens) [Neuroptera, Chrysopidae]. Son impact entomophage en verger d'oliviers. Thèse de Doctorat d’État. Université Paul-Sabatier. Toulouse. 227 pp.

Aspöck, H., 1973. Die Erforschung der Neuropteren Europas-Rückblick, Standortsbestimmung und Ziele. Zeitschrift der Arbeitsgemeinschaft Österreichischer Entomologen, 24: 2-30.

Aspöck, H., 1977. Die Herkunft der Raphidiopteren des Extramediterranen Europa. Eine kritische biogeographische Analyse. 7 Internationalen Symposiums über Entomofaunistik in Mitteleuropa, Leningrad, 1: 14-22.

Aspöck, H., 1979. The Raphidioptera of continental Greece: A chorological analysis. Biologia Gallo-Hellenica, 8: 243-261.

Aspöck, H., 1981. Die Erforschung der Neuropteren Europas-Ergebnisse und aktuelle Probleme. Mitteilungen der Deutschen Gesellschaft für Allgemeine und Angewandte Entomologie, 3: 179-182.

Aspöck, H., 1986. The Raphidioptera of the World: A Review of Present Knowledge. In: J. Gepp, H. Aspöck \& H. Hölzel (eds.). Recent Research in Neuropterology (Proceedings of the 2nd International Symposium on Neuropterology, Hamburg, 1984). Graz: 15-29.

Aspöck, H., 1987. The Raphidioptera of the Middle East: A Review: (Insecta: Neuropteroidea). In: F. Krupp, W. Schneider \& R. Kinzelbach (eds.). Proceedings of the Symposium on the Fauna and Zoogeography of the Middle East. Beihefte zum Tübinger Atlas des Vorderen Orients, Reihe A, 28: 148-159.

Aspöck, H., 1990. The Raphidioptera of Africa: a review of the present knowledge (Insecta: Neuropteroidea). In: M.W. Mansell \& H. Aspöck (eds.). Advances in Neuropterology (Proceedings of the Third International Symposium on Neuropterology, Berg en Dal, 1988). Pretoria: 47-61.

Aspöck, H., 1991. Grundlagen des möglichen Einsatzes von Raphidiopteren in der biologischen Schädlingsbekämpfung. Verhandlungen des XII Internationales Symposium über Entomofaunistik Mitteleuropa Verhandlungen, Kiev 1988. Akademie der Wissenschaften. Kiew: 26-33.

Aspöck, H., 1992. The Neuropteroidea of Europa: a review of present knowledge (Insecta). In: M. Canard, H. Aspöck \& M. W. Mansell (eds.). Current Research in Neuropterology (Proceedings of the Fourth International Symposium on Neuropterology, Bagnères-deLuchon, 1991). Toulouse: 43-56.

Aspöck, H., 1997. Flying power of Atlantoraphidia maculicollis Stephens (Raphidioptera: Raphidiidae). Entomologist's record and Journal of Variation, 109: 113-114. 
Aspöck, H., 1998a. Descriptions and illustrations of Raphidioptera in the early entomological literature before 1800, Acta Zoologica Fennica, 209: 7-31.

Aspöck, H., 1998b. Distribution and biogeography of the order Raphidioptera: updated facts and new hypothesis. Acta Zoologica Fennica, 209: 33-44.

Aspöck, H., 2000. Der endekreidezeitliche Impakt und das Überleben der Raphidiopteren. Entomologica Basiliensia, 22: 223-233.

Aspöck, H., 2002. The Biology of Raphidioptera: A review of the present knowledge. Acta Zoologica Academiae Scientiarum Hungaricae, 48, 2: 35-50.

Aspöck, H., 2003. Kamelhalsfliegen - lebende Fossilien: Eine der Endkreide-Katastrophe entkommende Tiergruppe. Verhandlungen Westdeutscher Entomologentag, 14: $1-6$

Aspöck, H. \& Aspöck, U., 1964. Synopsis der Systematik, Ökologie und Biogeographie der Neuropteren Mitteleuropas im Spiegel der Neuropteren-Fauna von Linz und Oberösterreich, sowie Bestimmungsschlüssel für die Mitteleuropäischen Neuropteren und Beschreibung von Coniopteryx lentiae nov. spec. Naturkundliches Jahrbuch der Stadt Linz, 1964: 127-282.

Aspöck, H. \& Aspöck, U., 1965. Zur Kenntnis der Raphidiiden von Südosteuropa und Kleinasien (Mit kritischen Bemerkungen zur Klassifikation der Familie). Annalen des Naturhistorischen Museums in Wien, 68: 309-364.

Aspöck, H. \& Aspöck, U., 1966a. Studien an europäischen und kleinasiatischen Arten des Genus Raphidia L. Mitteilungen der Schweizerischen Entomologischen Gesellschaft, 39: 33-48.

Aspöck, H. \& Aspöck, U., 1966b. Zur Kenntnis der Raphidiodea-Familie Inocellidae (Insecta, Neuroptera). Annalen des Naturhistorischen Museums in Wien, 69: 105-131.

Aspöck, H. \& Aspöck, U., 1967. Raphidiodea und Coniopterygidae (Planipennia) aus den zentralen und westlichen Teilen del Mongolei, (Insecta, Neuroptera). Ergebnisse der Mongolischen-Deutschen Expeditionen seit 1962, Nr 27. Mitteilungen aus dem Zoologischen Museum in Berlin, 43: 225-235.

Aspöck, H. \& Aspöck, U., 1968a. Agulla voluptaria nov. spec. und Agulla casta nov. spec. aus Spanien. Entomologisches Nachrichtenblatt, 15: 22-30.

Aspöck, H. \& Aspöck, U., 1968b. Vorläufige Mitteilung zur generischen Klassifizierung der Raphidiodea (Insecta, Neuroptera). Entomologisches Nachrichtenblatt, 15: 53-64.

Aspöck, H. \& Aspöck, U., 1968c. Neue subgenera des Genus Raphidia L. (Insecta, Raphidiodea) sowie drei weitere neue Arten dieser Gattung aus der Sowjet-Union (Voläufige Beschreibung). Entomologisches Nachrichtenblatt, 15: 89-92.

Aspöck, H. \& Aspöck, U., 1968d. Artbildung durch glazial bedingte Isolierung im Genus Raphidia L. (Insecta, Neuroptera). Annalen des Naturhistorischen Museums in Wien, 72: 21-27.

Aspöck, H. \& Aspöck, U., 1969a. Die Neuropteren Mitteleuropas. Ein Nachtrag zur "Synopsis der Systematik, Ökologie und Biogeographie der Neuropteren
Mitteleuropas”. Naturkundliches Jahrbuch der Stadt Linz, 1969: 17-68.

Aspöck, H. \& Aspöck, U., 1969b. Die Neuropteren Mitteleuropas. Eine faunistische zoogeographische Analyse. Abhandlungen und Berichte des Naturkundemuseums Görlitz, 44: 31-48.

Aspöck, H. \& Aspöck, U., 1971a. Ordnung Raphidioptera (Kamelhalsfliegen). In: J-G. Helmcke, D. Starck \& H. Wermuth (eds.). Handbuch der Zoologie 4, Arthropoda: Insecta. Walter de Gruter. Berlin und New York: 1-50.

Aspöck, H. \& Aspöck, U., 1971b. Inocellia (Reisserella n. subgen.) pasiphae n. sp. aus Kreta. (Ins. Raphidioptera, Inocellidae). Entomologische Zeitschrift, 81: 270-272.

Aspöck, H. \& Aspöck, U., 1971c. Das Subgenus Ohmella Aspöck et Aspöck (Neur., Raphidioptera, Raphidiidae, Raphidia L.). Zeitschrift der Arbeitsgemeinschaft Österreichischer Entomologen, 23: 25-32.

Aspöck, H. \& Aspöck, U., 1971d. Drei neue Raphidiiden-Spezies (Neuropteroidea - Raphidioptera). Nachrichtenblatt der Bayerischen Entomologen, 20: 86-88.

Aspöck, H. \& Aspöck, U., 1971e. Was ist Erma abdita Navás, 1918? (Ins., Neuropt., Raphidioptera). Zeitschrift der Arbeitsgemeinschaft Österreichischer Entomologen, 23: 107-113.

Aspöck, H. \& Aspöck, U., 1972a. Das Subgenus Subilla Navas Neur., Raphidioptera, Raphidiidae, Raphidia L.). Nachrichtenblatt der Bayerischen Entomologen, 21: 33-43.

Aspöck, H. \& Aspöck, U., 1972b. Das Subgenus Magnoraphidia Aspöck et Aspöck (Neur., Raphidioptera, Raphidiidae, Raphidia L.). Mitteilungen der Münchener Entomologischen Gesellschaft, 62: 13-30.

Aspöck, H. \& Aspöck, U., 1973a. Festlegung von Typen der Gattungsgruppe in der Familie Raphidiidae (Ins., Raphidioptera). Entomologische Zeitschrift, 83: 52-53.

Aspöck, H. \& Aspöck, U., 1973b. Inocellia (Amurinocellia n. subg.) calida n.sp. - eine neue Spezies der Familie Inocellidae (Ins., Raphidioptera) aus Ostasien (Mit einer Übersicht über die Inocelliden Asiens). Entomologische Berichten, 33: 91-96.

Aspöck, H. \& Aspöck, U., 1974a. Zur Taxonomie von Raphidia formosana Okamoto, 1917 (Ins., Neur., Raphidioptera). Entomologische Berichten, 34: 96-101.

Aspöck, H. \& Aspöck, U., 1974b. Raphidia (Subilla) xylidiophila n.sp. - Eine neue Pontomediterrane Kamelhalsfliege (Ins., Neuropt., Raphidioptera). Zeitschrift der Arbeitsgemeinschaft Österreichischer Entomologen, 25: 111-113.

Aspöck, H. \& Aspöck, U., 1974c. Der Typus von Raphidia physodes NAVAS, 1914 - ein Monstrum? (Ins., Raphidioptera, Raphidiidae). Revue Suisse de Zoologie, 81: 177-188.

Aspöck, H. \& Aspöck, U., 1974d. Zwei neue Raphidiiden - Species aus Griechenland (Neuropt., Raphidioptera). Entomologische Zeitschrift, 84: 165-169.

Aspöck, H. \& Aspöck, U., 1975. Zur Taxonomie von Raphidia (Iranoraphidia n. subg.) wittmeri Asp. et Asp. (Neur., Raphidioptera, Raphidiidae). Zeitschrift der Arbeitsgemeinschaft Österreichischer Entomologen, 27: 16-18.

Aspöck, H. \& Aspöck, U., 1976. Die Auflösung des Raphidia notata - Komplexes (Neur., Raphidioptera, 
Raphidiidae). Zeitschrift der Arbeitsgemeinschaft Österreichischer Entomologen, 27: 57-70.

Aspöck, H. \& Aspöck, U., 1977. Raphidia (Ohmella) postulata n. sp. eine neue Kamelhalsfliege aus Spanien (Neuropteroidea, Raphidioptera). Entomologische Zeitschrift, 87: 185-189.

Aspöck, H. \& Aspöck, U., 1985. Inocellia taiwana n. sp. - eine neue Inocelliiden-Spezies aus Taiwan. Entomologische Zeitschrift mit Insektenbörse, 95: 45-48.

Aspöck, H. \& Aspöck, U., 1991. Raphidioptera (Snakeflies, camelneck- flies). In: I. D. Naumann \& P. B. Carne (eds.). The insects of Australia. A textbook for students and research workers. Second edition, Vol I. Melbourne University Press. Melbourne: 521-524.

Aspöck, H. \& Aspöck, U., 1994. Raphidioptera (snake-flies, camelneck-flies). In: I. D. Naumann (ed.). Systematic and applied Entomology - An Introduction. Melbourne University Press. Melbourne: 338-339.

Aspöck, H. \& Aspöck, U., 2007. The Raphidioptera of the Apennines Peninsula: a biogeographical analysis. Annali del Museo Civico di Storia Naturale di Ferrara, [2005], 8: 95-106.

Aspöck, H. \& Aspöck, U., 2009. Raphidioptera - Kamelhalsfliegen. Ein Überblick zun Einstieg. Entomologica Austriaca, 165: 53-72.

Aspöck, H. \& Aspöck, U., 2012. Raphidioptera. In: Encyclopedia of Science \& Technology, 11th ed., Volume 15. McGraw-Hill. New York: 217-219.

Aspöck, H. \& Aspöck, U., 2013. Woher kommen die Namen? Die validen rezenten Taxa der Kamelhalsfliegen der Erde: Systematisches Verzeichnis und Etymologie (Insecta: Endopterygota: Neuropterida: Raphidioptera). Entomologica Austriaca, 20: 9-155.

Aspöck, H., Aspöck, U. \& Hölzel, H., 1980a. Die Neuropteren Europas. Eine zusammenfassende Darstellung der Systematik, Ökologie und Chorologie der Neuropteroidea (Megaloptera, Raphidioptera, Planipennia) Europas. 2 Bande. Goecke und Evers. Krefeld. 495 pp.; 355 pp.

Aspöck, H., Aspöck, U. \& Martynova, O., 1969. Untersuchungen über die Raphidiiden-Fauna der Sowjet-Union (Insecta, Raphidioptera). Tijdschrift voor Entomologie, 112: 123-164.

Aspöck, H., Aspöck, U. \& Rausch, H., 1974a. Bestimmungsschlüssel der Larven der Raphidiopteren Mitteleuropas (Insecta, Neuropteroidea). Zeitschrift für Angewandte Zoologie, 61: 45-62.

Aspöck, H., Aspöck, U. \& Rausch, H., 1974b. Das Subgenus Venustoraphidia Asp. et Asp. (Neur., Raphidioptera, Raphidiidae, Raphidia L.). Zeitschrift der Arbeitsgemeinschaft Österreichischer Entomologen, 25: 101-111.

Aspöck, H., Aspöck, U. \& Rausch, H., 1975. Raphidiopteren - Larven als Bodenbewohner. (Mit Beschreibung der Larven von Ornatoraphidia, Parvoraphidia und Superboraphidia). Zeitschrift für Angewandte Zoologie, 62: 361-375.

Aspöck, H., Aspöck, U. \& Rausch, H., 1980b. Raphidia (Dichrostigma?) santuzza n. sp. - eine neue Kamelhalsfliege aus Kalabrien. Entomologische Zeitschrift, 90: 73-79.
Aspöck, H., Aspöck, U. \& Rausch, H., 1982. Drei neue Raphidiiden - Spezies aus Anatolien. Nachrichtenblatt der Bayerischen Entomologen, 31: 78-90.

Aspöck, H., Aspöck, U. \& Rausch, H., 1983a. Phaeostigma (Ph.) promethei n.sp. - eine neue Raphidiiden Spezies aus dem Kaukasus. Nachrichtenblatt der Bayerischen Entomologen, 32: 114-118.

Aspöck, H., Aspöck, U. \& Rausch, H., 1983b. Mauroraphidia maghrebina n. gen. n. sp. - eine neue Raphidiiden - Spezies aus dem Atlas. Zeitschrift der Arbeitsgemeinschaft Österreichischer Entomologen, 35: 27-32.

Aspöck, H., Aspöck, U. \& Rausch, H., 1984. Turcoraphidia helthitica n.sp. - eine neue Raphidiiden Spezies aus Anatolien. Nachrichtenblatt der Bayerischen Entomologen, 33: 97-106.

Aspöck, H., Aspöck, U. \& Rausch, H., 1985a. Parasiten und Parasitierung bei Raphidiopteren - eine Übersicht des gegenwärtigen Forschunstandes. Mitteilungen der Deutschen Gesellschaft für Allgemeine und Angewandte Entomologie, 4: 386-388.

Aspöck, H., Aspöck, U. \& Rausch, H., 1985b. Zur Kenntnis der genera Tjederiraphidia n.g. und Mongoloraphidia H.A \& U.A. Z. Zeitschrift der Arbeitsgemeinschaft Österreichischer Entomologen, 37: 37-48.

Aspöck, H., Aspöck, U. \& Rausch, H., 1989. The Raphidioptera of the eastern Mediterranean: a zoogeographical analysis. Biologia Gallo-Hellenica, 15: 67-111.

Aspöck, H., Aspöck, U. \& Rausch, H., 1991. Die Raphidiopteren der Erde. Eine monographische Darstellung der Systemtatik, Taxonomie, Biologie, Ökologie und Chorologie der rezenten Raphidiopteren der Erde, mit einer zusammenfassenden Übersicht der fossilen Raphidiopteren (Insecta: Neuropteroidea). 2 Bande. Goecke und Evers. Krefeld. 730 pp.; 550 pp.

Aspöck, H., Aspöck, U. \& Rausch, H., 1995. Untersuchungen über die Raphidiopteren von Kirgistan: Übersicht der nachgewiesenen Arten und Beschreibung von vier neuen Spezies. Entomologische Nachrichten und Berichte, 39: 165-182.

Aspöck, H., Aspöck, U. \& Rausch, H., 1996. Weitere Untersuchungen über die Raphidiiden von Kirgistan: Beschreibung von sechs neuen Spezies (Insecta: Neuropteroidea: Raphidioptera: Raphidiidae). Entomologische Nachrichten und Berichte, 40: 193-215.

Aspöck, H., Aspöck, U. \& Rausch, H., 1997. Erstnachweis der Ordnung Raphidioptera in Turkmenistan und weitere neue Raphidiiden-Spezies aus Kirgisistan (Insecta: Neuropteroidea: Raphidioptera: Raphidiidae). Entomologische Nachrichten und Berichte, 41: 77-95.

Aspöck, H., Aspöck, U. \& Rausch, H., 1998a. Was ist Usbekoraphidia turkestanica (H. Aspöck, U. Aspöck \& Martynova 1968)? Zur Kentniss der Taxonomie, Ökologie und Chorologie mittelasiatischer Raphidiiden (Insecta: Raphidioptera, Raphidiidae). Stapfia, 55: 421-457.

Aspöck, H., Aspöck, U. \& Rausch, H., 1999. Biologische und chorologische Charakterisierung der Raphidiiden der östlichen Paläarktis und Verbreitungkarten der in Kasachstan, Kirgistan, Usbekistan, Turkmenistan und Tadschikistan nachgewiesenen Arten der Familie 
(Neuropterida: Raphidioptera: Raphidiidae). In: H. Aspöck (ed.). Neuropterida: Raphidioptera, Megaloptera, Neuroptera, Kamelhälse, Schlammfliegen, Ameisenlöwen. Stapfia, Neue Folge, 138: 59-84.

Aspöck, H., Aspöck, U. \& Yang, Ch. K., 1998b. The Raphidiidae of Eastern Asia (Insecta, Neuropterida, Raphidioptera). Deutsche Entomologische Zeitschrift, 45: 115-128.

Aspöck, H. \& Hölzel, H., 1996. The Neuropteroidea of North Africa, Mediterranean Asia and of Europe: a comparative review (Insecta). In: M. Canard, H. Aspöck, H. \& M. W. Mansell (eds.). Pure and Applied Research in Neuropterology (Proceedings of the Fifth International Symposium on Neuropterology, Cairo, 1994). Toulouse: 31-86.

Aspöck, H., Hölzel, H. \& Aspöck, U., 2001. Kommentierter Katalog der Neuropterida (Insecta: Raphidioptera, Megaloptera, Neuroptera) der Westpaläarktis. Denisia, 2: 1-606.

Aspöck, H., Liu, X. \& Aspöck, U., 2012. The family Inocelliidae (Neuropterida: Raphidioptera): A review of present knowledge. Mitteilungen der Deutschen Gesellschaft für Allgemeine und Angewandte Entomologie, 18: 565-573.

Aspöck, H., Rausch, H. \& Aspöck, U., 1974c. Untersuchungen über die Ökologie der Raphidiopteren Mitteleuropas (Insecta, Neuropteroidea). Zeitschrift für Angewandte Entomologie, 76: 1-30.

Aspöck, U., 1974. Die Raphidiopteren der Nearktis (Insecta, Neuropteroidea). Dissertation. Universität Wien. 2 Bande. 238 pp.; 285 pp.

Aspöck, U., 1975. The present state of knowledge on the Raphidioptera of America (Insecta, Neuropteroidea). Polskie Pismo Entomologiczne, 45: 537-546.

Aspöck, U., 1979. The Raphidioptera of the Aegean Islands: a chorological analysis. Biologia Gallo-Hellenica, 8: 265-275.

Aspöck, U., 1981. Die Raphidiopteren der Erde - eine zoogeographische Analyse. Mitteilungen der Deutschen Gesellschaft für Allgemeine und Angewandte Entomologie, 3: 171-173.

Aspöck, U., 1991. Der gegenwärtige Stand der Erforschung der Rhaphidiopteren der Sowjetunion. Verhandlungen des 12 Internationalen Symposiums über Entomofaunistik Mitteleuropas, Kiew, 1988. Akademie der Wissenschaften. Kiew: 239-244.

Aspöck, U., 1995. Neue Hypothesen zum System der Neuropterida. Mitteilungen der Deutschen Gesellschaft für Allgemeine und Angewandte Entomologie, 10: 633-636.

Aspöck, U. \& Aspöck, H., 1969. Die Raphidiiden Africas (Insecta, Raphidioptera). Annalen des Naturhistorischen Museums in Wien, 73: 161-169.

Aspöck, U. \& Aspöck, H., 1970. Untersuchungen über die Raphidiopteren Mexikos (Insecta, Neuropteroidea). Polskie Pismo Entomologiczne, 40: 705-725.

Aspöck, U. \& Aspöck, H., 1990. Xanthostigma gobicola n. sp. und Mongoloraphidia (Alatauroraphidia) medvedevi n. sp. - zwei neue Raphidiiden Species aus Zentralasien. Zeitschrift der Arbeitsgemeinschaft Österreichischer Entomologen, 42(3/4): 97-104.
Aspöck, U. \& Aspöck, H., 1991. Mongoloraphidia (Hissaroraphidia) kelidotocephala n. sp. und Mongoloraphidia (Alautoraphidia) dolinella n. sp. - zwei neue Raphidiiden - Spezies aus Südost - Kasachstan. (Neuropteroidea: Raphidioptera: Raphidiidae). Zeitschrift der Arbeitsgemeinschaft Östereichischer Entomologen, 43: 25-32.

Aspöck, U. \& Aspöck, H., 1994. Paradoxe Verbreitungsbilder von Neuropteroidea (Insecta: Raphidioptera, Neuroptera). Zeitschrift der Arbeitsgemeinschaft Österreichischer Entomologen, 46(1/2): 30-44.

Aspöck, U. \& Aspöck, H., 1996. Raphidioptera. In: J. E. L. Bousquets, A. N. G. Aldrete \& E. G. Soriano (eds.). Biodiversidad, Taxonomía y Biogeografía de Artrópodos de México: Hacia una síntesis de su conocimiento, vol. 19. D.R. Universidad Autónoma de México. México D.F.: 277-286.

Aspöck, U. \& Aspöck, H., 1999a. Kamelhälse, Schlammfliegen, Ameisenlöwen. Wer sind sie? (Insecta: Neuropterida: Raphidioptera, Megaloptera, Neuroptera). Stapfia, 60(138): 1-34.

Aspöck, U. \& Aspöck, H., 1999b. Was ist Sininocellia gigantos YANG 1985? (Neuropterida: Raphidioptera: Inoccellidae). Linzer Biologische Beitrage, 31(1): 487-492.

Aspöck, U., Aspöck, H. \& Rausch, H., 1992. Rezente Südgrenzen der Ordnung Raphidioptera in Amerika. Entomologia Generalis, 17: 169-184. http://dx.doi. org/10.1127/entom.gen/17/1992/169

Aspöck, U., Aspöck, H. \& Rausch, H., 1994a. Die Kopulation der Raphidiopteren: Eine zusammenfassende Übersicht des gegenwärtigen Wissenstandes. (Insecta: Neuropteroidea). Mitteilungen der Deutschen Gesellschaft für Allgemeine und Angewandte Entomologie, 9: 393-402.

Aspöck, U., Aspöck, H. \& Rausch, H., 1994b. Neue Arten der Familie Raphidiidae aus Mexiko und Nachweis der Spermatophore in der Ordnung Raphidioptera. Entomologia Generalis, 18: 145-163. http://dx.doi.org/10.1127/ entom.gen/18/1994/145

Aspöck, U., Haring, E. \& Aspöck, H., 2012. Biogeographical implications of a molecular phylogeny of the Raphidiidae (Raphidioptera). Mitteilungen der Deutschen Gesellschaft für Allgemeine und Angewandte Entomologie, 18: 575-582.

Aspöck, U., Plant, J. D. \& Nemeschkal, H. L., 2001. Cladistic analysis of Neuroptera and their systematic position within Neuropterida (Insecta: Holometabola: Neuropterida: Neuroptera). Systematic Entomology, 26: 73-86. http://dx.doi.org/10.1046/j.1365-3113.2001.00136.x

Ast, F., 1920. Über den feineren Bau der Facettenaugen bei Neuropteren. Zoologische Jahrbücher. Abteilung für Anatomie und Ontogenie der Tiere, 4: 411-458.

Aubert, J. F., 1961. Révision des travaux concernant les Ichneumonides de France et 3 me supplement au catalogue de Gaulle (80 éspèces nouvelles pour la faune française). Bulletin Mensuel de la Société Linnéenne de Lyon, 30: 195-200.

Banks, N., 1892. A synopsis, catalogue, and bibliography of the neuropteroid insects of temperate North America. 
Transactions of the American Entomological Society, 19: 327-373.

Banks, N., 1923. Venational variation in Raphidia. The Canadian Entomologist, 54: 114-116. http://dx.doi. org/10.4039/Ent54114-5

Barnard, P. C., 1988. The genus Raphidia. Provisional key to British species of Raphidia Linnaeus. Neuro News, August, 2: 3-11.

Barnard, P. C., Brooks, S. J. \& Stork, N. E., 1986. The seasonality and distribution of Neuroptera, Raphidioptera and Mecoptera on oaks in Richmond Park, Surrey, as a revealed by insecticide knock-down sampling. Journal of Natural History, 20: 1321-1331. http://dx.doi. org/10.1080/00222938600770881

Bartoš, E., 1952. Dlouhošijky sbírek entomologického musea v Brně (Raph.). Časopis Československé Společnosti Entomologické, 49: 214-221.

Bartoš, E., 1964. Ergebnisse der Albanien-Expedition 1961 des Deutschen Entomologischen Institutes. Beiträge zur Entomologie, 14: 319-322.

Bartoš, E., 1965a. Agulla trilobata nov. spec. und Bemerkungen zu verwandten Arten. Reichenbachia, 5: 87-99.

Bartoš, E., 1965b. Agulla rostrata sp. n. aus Moldavien (UdSSR). Acta Entomologica Bohemoslovaca, 62: 458-467.

Bartoš, E., 1965c. Raphidia schizurotergalis n. sp., eine neue Raphidien - Art aus der Moldawischen SSR. Acta Entomologica Bohemoslovaca, 62: 129-138.

Bartoš, E., 1965d. Eine neue Kamelhalsfliege aus der CSSR - Raphidia barbata n. sp. Acta Entomologica Bohemoslovaca, 62: 228-232.

Bartoš, E., 1967. Die Raphidiopteren der Entomologischen Abteilung des Naturwisenschaftlichen Museum in Praha. Acta Entomologica Musei Nationalis Pragae, 37: 325-345.

Bastero Monserrat, J. J., 1989. Longinos Navás, científico jesuita. Universidad de Zaragoza. Zaragoza. 229 pp.

Berland, L., 1962. Atlas des Névroptères de Francia, Belgique, Suisse. Nouvel Atlas d'Entomologie, 5. N. Boubée \& Cie. Paris. 158 pp.

Berland, L. \& Grassé, P. P., 1951. Ordre des Raphidioptères (Raphidiinae Latreille, 1810; Raphidides Leach, 1815). In: P.-P. Grassé. Traité de Zoologie, vol. 10 (Insectes supérieurs et Hémiptéroides). Masson et Cie. Paris: 18-22.

Billberg, G. J., 1820. Enumeratio Insectorum in Museo Gust. Joh. Billberg. Typis Gadelianis. Stockholm. 158 pp.

Blair, K. G., 1951. Neuroptera of the Isle of Wight. Proceedings of the Isle of Wight Natural History (and Archaeological) Society, 4: 157-162.

Blanchard, E., 1840. Histoire naturelle des Insectes Orthoptères, Névroptères, Hémiptères, Hyménoptères et Diptères; avec une introduction renfermant l'anatomie et la physiologie des animaux articulés. Volume 3. P. Duménil. Paris. 672 pp.

Bohigas, M. \& Sanchez, A., 1917. Catáleg dels Insectes del Museu pertanyents als ordres: ParaneuropteraEphemeroptera-Plecoptera-Neuroptera-MegalopteraRhaphidioptera-Embioptera-Mecoptera-Psocoptera-
Trichoptera. Anuari-Junta de Ciències Naturals de Barcelona, 2(1): 301-326.

Boudreaux, H. B., 1979. Arthropod phylogeny with special reference to insects. John Wiley \& Sons. New York. $320 \mathrm{pp}$.

Brauer, F., 1852. Versuch einer Gruppirung der Gattungen in der Zunft Planipennia mit besonderer Rücksicht auf die früheren Stände. Stettiner Entomologische Zeitung, 13: 71-77.

Brauer, F., 1855a. Beiträge zur Kenntniss des inneren Baues und der Verwandlung der Neuropteren. Verhandlungen des Zoologisch-Botanischen Vereins in Wien, 5: 701-726.

Brauer, F., 1855b. Beiträge zur Kentniss der Verwandlung der Neuropteren. Verhandlungen des Zoologische-Botanischen Vereins in Wien, 5: 479-484.

Brauer, F., 1876. Die Neuropteren Europas und insbesondere Östereichs mit Rücksicht auf ihre geographische Verbreitung. Festschrift zur Feier des fünfundzwanzigjährigen Bestehen Kaiserlich-Koniglichen ZoologischBotanischen Gesellschaft: 263-300.

Brauer, F., 1878. Verzeichniss der Neuropteren Deutschlands und Östereichs. Verzeichnis der Neuropteren Deutschlands und Österreichs. Auszug aus "Die Neuropteren Europas.” Entomologische Nachrichten, 4: 69-74, 85-90.

Brauer, F., 1886. Neuroptera genuina (Netzflügler). In: G. Beck (ed.). Fauna von Hernstein in Niederösterreich und der weiteren Umgebung, Bande II(2). A. Holzhausen. Wien: 21-27.

Brauer, F. \& Löw, F., 1857. Neuroptera austriaca. Die im Erzherzogthum Oesterreich bis jetzt aufgefundenen Neuropteren nach der analytischen Methode zusammengestellt, nebst einer kurzen Charakteristik aller europäischen Neuropteren-Gattungen. C. Gerold's Sohn. Wien. 80 pp.

Briggs, C. A., 1906. The recent Neuroptera of Devonshire. Report and Transactions - Devonshire Association for the Advancement of Science, Literature and Art, 38: 357-369.

Brongniart, C. H., 1893. Recherches pour servir à l'histoire des insectes fossiles des temps primaires précédées d'une étude sur la nerviation des ailes des insectes. Ètudes sur le terrain houiller de commentry. Théolier \& Cie. Saint-Etienne. 493 pp.

Browning, F. R., 1948. An additional europan spruce sawfly (Hym. Tenthredinidae) distinguished from Pristophora ambigua (Fallen) and ocurring in Britain. The Entomologist's Monthly Magazine, 84: 163.

Brues, C. T., Melander, A.L. \& Carpenter, F.M., 1954. Classification of Insects. Bulletin of the Museum of Comparative Zoology, 108: 1-917.

Büchs, W., 1988. Stamm-Rindenzoozönosen verschiedener Baumarten des Hartholzauenwaldes und ihr Indikatorwert für die Früherkennung von Baumschäden. Dissertation Mathematisch-naturwissenschaftliche. Rheinische Friedrich-Wilhelms-Universität. Bonn. 813 pp.

Büning, J., 1980. The Ovary of Raphidia flavipes is Telotrophic and of the Sialis Type (Insecta, Raphidioptera). Zoomorphologie, 95: 127-131. 
Büning, J., 1994. The Insect ovary. Ultraestructure, previtellogenic growth and evolution. Chapmann \& Hall. London. 400 pp.

Buresch, I. \& Popov, A., 1973. Ausländische Literatur über die Fauna Bulgariens. Izvestiâ na Zoologičeskiâ institut s muzej, 37: 151-156.

Burmeister, H., 1829. De Insectorum systemate naturali. Dissertatio inauguralis quam consensu facultatis medicae halensis. Grunert \& Fil. Halle. 43 pp.

Burmeister, H., 1837. Handbuch der Naturgeschichte. Zum Gebrauch bei Vorlesungen. Zweite Abteilung. Zoologie, T. C. Enslin. Berlin. 858 pp.

Burmeister, H., 1839. Handbuch der Entomologie. 2 Bande. Besondere Entomologie, 2. Abteilung Kakerfe. Gymnognatha (2. Hälfte; vulgo Neuroptera). T. C. Enslin. Berlin. pp. 757-1050.

Campion, H., 1915. Some observations on the Life-History of Snake-Flies (Raphidia: Order Planipennia). The Entomologist's Monthly Magazine, 51: 24-26.

Capra, F., 1976. Quanto si sa sugli Odonati e Neurotteri dell'Arcipelago Toscano. (Studi sulla Riserva Naturale dell'Isola di Montecristo. 13). Lavori della Societa Italiana di Biogeografia, 5: 541-560.

Carpenter, F. M., 1936. Revision of the Neartic Raphidiodea (Recent and Fossil). Proceedings of the American Academy of Arts and Sciences, 71: 89-157. http://dx.doi. org/10.2307/20023217

Carpenter, F. M., 1958. Mexican Snake-Flies (Neuroptera:Raphidiodea). Psyche, 65: 52-58. http://dx.doi. org/10.1155/1958/14859

Carpenter, F. M., 1967. Cretaceous Insects from Labrador 2. A new Family of Snake - Flies (Neuroptera: Alloraphidiidae). Psyche, 74: 270-275. http://dx.doi. org/10.1155/1967/62569

Carpenter, F. M., 1992. Part R, Arthropoda 4. Volume 4: Superclass Hexapoda. In: R. L. Kaesler (ed.). Treatise on invertebrate paleontology. The Geological Society of America and The University of Kansas. Lawrence: 279-655.

Carr, J. W., 1914. The Neuroptera of Nottingham. The Entomologist, 47: 66-68.

Castellani, O., 1957. Contributo alla conoscenza della fauna entomologica d' Italia. Bollettino dell'associazione romana di entomologia, 11-12(3-4): 9-14.

Cederhielm, J., 1798. Faunae ingricae proromus exhibens methodicam descriptionem insectorum agri petropolensis praemissa, mammalium, avium, amphiniorum et piscium enumeratione. J. F. Hartknoch. Lipsiae. $348 \mathrm{pp}$.

Chládek, F. \& Laštůvka, Z., 1982. K poznání dlouhošíjek (Raphidioptera) Moravy. Acta Universitatis Agriculturae et Silviculturae Mendelianae Brunensis, 30: 65-68.

Clausen, C. P., 1940. Entomophagus Insects. McGraw Hill Book Company, Inc., New York and London, 688 pp.

Cockerell, T. D. A., 1912. A Fossil Raphidia (Neur., Planip.). Entomological News, 23: 215-216.

Cockerell, T. D. A. \& Custer, C., 1925. A New Fossil Inocellia (Neuroptera) from Florissant. Entomologist, 58: 295-297.
Cocquemont, C. \& Chambon, J. P., 1990. Contribution à l'étude de la faune entomologique du parc national du mercantour et ses environs inmediats (Neuroptera, Raphidioptera, Planipennia, Orthoptera, Dermaptera, Lepidoptera, Coleoptera, Hymenoptera, Hemiptera, Homoptera, Diptera). Biocosme Mésogéen, 7(3-4): 133-150.

Collins, G.A., 1989. Raphidia maculicollis Stephens in Surrey. Neuro News, 4: 4.

Comstock, J. H., 1918. The wings of Insects. Comstock Publishing. Ithaca. $430 \mathrm{pp}$.

Condé, B. \& Pagès, J., 1954. Raphidiopteres du Musee Zoologique de Strasbourg. Bulletin de l'Association Philomathique d'Alsace et de Lorraine, 9: 142-145.

Condé, B. \& Pagès, J., 1956a. Stations francaises de Raphidioptères. Bulletin de la Société Entomologique de France, 61: 125-132.

Condé, B. \& Pagès, J., 1956b. Contribution a la Fauna de la Bourgogne. Travaux du Laboratoire de Zoologie et de la Station Aquicole Grimaldi de la Faculté des Sciences de Dijon, 14: 1-2.

Condé, B. \& Pagès, J., 1959. Captures récentes de Raphidioptères en France. Bulletin de la Société Entomologique de France, 64: 191-193.

Costa, A., 1855. Fauna del Regno di Napoli ossia enumerazione di tutti gli animali che abitano le diverse regioni di questo regno e le acque che le bagnano e descrizione de' nuovi o poco esattamente conosciuti con figure ricavate da originali viventi e dipinte al naturale. Stamperia di Antonio Cons. Napoli.

Craik, J. C. A., 1980. Records of some Non-Lepidopteran Insects from the New Forest Area. The Entomologist's Record and Journal Variation, 92(10): 243-246.

Crampton, G. C., 1918. The genitalia and terminal abdominal structures of male Neuroptera and Mecoptera with notes on the Psocidae, Diptera and Trichoptera. Psyche, 25: 48-59. http://dx.doi.org/10.1155/1918/78139

Crampton, G. C., 1929. The terminal abdominal structures of female insects compared troughout the orders from the standpoint of Phylogeny. Journal New York Entomological Society, 37(4): 453-511.

Cross, I., 2000. Oviposition by Atlantoraphidia maculicollis. Neuro News, 26: 26.

Curtis, J., 1824. British Entomology; being illustrations and descriptions of the genera of insects found in Great Britain and Ireland containing coloured figures from nature of the most rare and beautiful species, and in many instances of the plants upon which they are found, Vol. 1. London. 50 Pl.+Text.

Curtis, J., 1837. A guide to an arrangement of British insects; being a catalogue of all the named species hitherto discovered in Great Britain and Ireland. $2^{\text {nd }}$ edition. J. Pigot \& Co. London. 294 pp.

Czechowska, W., 1982. Neuroptera and Mecoptera of Warsaw and Mazovia. Memorabilia Zoologica, 36: 165-184.

Czechowska, W., 1985. Neuropteran (Planipennia and Raphidioptera; Neuropteroidea) communities of coniferous forests in the Kampinoska Forests and in Bialoleka Dworska near Warsaw. Fragmenta Faunistica, 29: 391-404. 
Czechowska, W., 1986. Structure of Neuropteran communities in urban green areas of Warsaw. Memorabilia Zoologica, 41: 187-214.

Czechowska, W., 1990. Neuropterans (Neuropteroidea) of linden-oak-hornbeam and therophilous oak forests of the Mazovian Lowland. Fragmenta Faunistica, 34: 7.

Czechowska, W., 1994. Neuropterans (Neuropteroidea: Raphidioptera, Planipennia) of the canopy layer in pine forests. Fragmenta Faunistica, 36: 459-467.

Czechowska, W., 1997. A comparative analysis of the structure of Neuropteroidea communities of tree canopies in linden-oak-hornbeam forests, light oak forests, mixed coniferous forests and pine forests. Fragmenta Faunistica, 40: 127-168.

Czechowska, W. \& Dobosz, R., 1991. 20. Raphidioptera. In: J. Razowzki. Wykoz zwievzgt Polski, Vol. 1. Ossolineum. Wroclaw: 1-20.

Czihak, G., 1952. Skelett and Muskulatur des Thorax von Raphidia flavipes Stein. (Ein Beitrag zur vergleichende Anatomie des Neuropterenthorax). Dissertatio. Universität Wien. 70 pp.

De Geer, K. 1779. Abhandlungen zur Geschichte der Insekten. Aus dem Französichen übersetzt und mit Anmerkungen herausgegeben von J.A.E.Götze. Bande 2(2). G.N. Raspe. Nürnberg. 384 pp.

Dettner, K. \& Peters, W., 1999. Übersicht über die Vielfalt der Insekten. In: Lehrbuch der Entomologie, 1 Auflage. Gustav Fischer Verlag. Stuttgart, Jena, Lübeck und Ulm: 763-903.

Devetak, D., 1980. A Contribution to the knowledge of Megaloptera, Raphidioptera and Neuroptera in Slovenia (Yugoslavia). In: J. Gepp, H. Aspöck \& H. Hölzel (eds.). Progress in World's Neuropterology (Proceedings of the 1st International Symposium on Neuropterology, Graz, 1980). Graz: 69-70.

Devetak, D., 1984a. Megaloptera, Raphidioptera and Planipennia in Slovenia (Yugoslavia). Faunistical contribution. Neuroptera International, 3: 55-72.

Devetak, D., 1984b. A contribution to the Knowledge of Megaloptera, Raphidioptera and Neuroptera in Slovenia (Yugoslavia). In: J. Gepp, H. Aspöck \& H. Hölzel (eds.). Progress in World's Neuropterology (Proceedings of the 1st International Symposium on Neuropterology, Graz, 1980). Graz: 69-70.

Devetak, D., 1992a. Physiology of neuropteran vibration receptors: Chrysoperla carnea (Stephens) as an example (Insecta: Neuroptera: Chrysopidae). In: M. Canard, H. Aspöck \& M. W. Mansell (eds.). Current Research in Neuropterology (Proceedings of the 4th International Symposium on Neuropterology, Bagnères-de-Luchon, 1991). Toulouse: 105.

Devetak, D., 1992b. Megaloptera, Raphidioptera and Planipennia (Neuropteroidea, Insecta) of Croatia. Znanstvena Revija, 1(4): 89-114.

Devetak, D., 1992c. Present knowledge of the Megaloptera, Raphidioptera and Neuroptera of Yugoslavia (Insecta: Neuropteroidea). In: M. Canard, H. Aspöck \& M. W. Mansell (eds.). Current Research in Neuropterology (Proceedings of the 4th International Symposium on Neuropterology, Bagnères-de-Luchon, 1991). Toulouse: 107-118.
Díaz-Aranda, L. M. \& Monserrat, V. J., 1988a. Contribución al conocimiento de los neurópteros de Teruel (Insecta, Neuropteroidea). Boletín de la Asociación española de Entomología, 12: 215-231.

Díaz-Aranda, L. M. \& Monserrat, V. J., 1988b. Contribución al conocimiento de los Neurópteros de Granada (Insecta, Neuropteroidea). Actas del tercer Congreso Ibérico Entomología, Granada, 1988: 211-288.

Díaz-Aranda, L. M. \& Monserrat, V. J., 1988c. Contribución al conocimiento de los neurópteros de Jaén (Insecta, Neuropteroidea). Boletín de la Asociación española de Entomología, 12: 111-123.

Díaz-Aranda, L. M., Monserrat, V. J. \& Marín, F., 1986. Contribución al conocimiento de los neurópteros de Guadalajara (Insecta, Neuropteroidea). Actas de las octavas Jornadas Asociación española de Entomología, Sevilla: 1131-1144.

Dobosz, R., 1989. Siatkoskrzydle (Neuropteroidea) w zbiorach Muzeum Górnóslaskiego w Bytomiu. Annals of the upper Silesian Museum in Bytom Natural History, 12: 75-89.

Dobosz, R., 1990. Stan Pozonia Neuropteroidea Ojocowskiego Parku Narodowego. Pradnik Prace i Materialy Muzeum im Prof. Wladyslawa Szafera, 1: 63-64.

Dobosz, R., 1991a. Snake-flies (Raphidioptera, Neuroptera) of Poland - A faunistic Review. Entomologia, 2: 191-208.

Dobosz, R., 1991b. Neuropteroidea in the collection of the Institute of Animal Systematics and Evolution pas in Cracow. Annals of the Upper Silesian Museum (Entomology), 2: 221-233.

Dobosz, R., 1994. Materialy do fauny wielbladek (Neuropteroidea: Raphidioptera) Polski. Acta Entomologica Silesiana, 2(1): 23.

Dobosz, R., Werstak, K. \& Czechowska, W., 2005. Raphidioptera of the Pieniny Mountains (S Poland). Annals of Upper Silesian Museum (Entomology), 13: 71-75.

Dorokhova, G. I., 1987. Otrjad Raphidioptera Verbljudki. In: G. S. Medvedev (ed.). Predelitel' Nasekomykh Evropeiskoi Chasti SSSR. Tom 4. 6. Chast' Bol'shekrylye, verblyudki, setchatokryle, skorpionovye mukhi, rucheiniki. Izdatel'stvo "Nauka". Leningrad: 27-35.

Douglas, J. W., 1859. Raphidia ---? [Bemerkungen über Entwicklung von Raphidiiden Larven]. Transactions of the Entomological Society of London, New Series, 5: 69.

Dubois, E. R., 1899. Notes sur l’habitat des Pseudo-Névroptéres et Névroptéres de la Gironde. Feuille des Jeunes Naturalistes, 3ème série, 29(339): 50-53.

Dziedzielewicz, J., 1867. Wykaz owadów siatkoskrzydłych (Neuroptera). Sprawozdania. Komisji Fizyograficzna, 1: 158-165.

Dziedzielewicz, J., 1891. Prezeglad fauny Krajowez owadów siatkoskrzydłych (Neuroptera, Pseudoneuroptera). Komisji Fizyograficzna, 26: 26-150.

Dziedzielewicz, J., 1895. Zestawienie zapisków o owadach siatkoskrzydłych w Tarech podezas pobytu w latach 1891 i 1892. Sprawozdania. Komisji Fizyograficzna, 30: 1-40.

Dziedzielewicz, J., 1905. Sieciarki (Neuroptera genuina) i Prasiatnice (Archiptera) zebrane w ciagu lat 1902 i 1903. Sprawozdania. Komisji Fizyograficzna, 38: 104-125. 
Dziedzielewicz, J., 1920. 7. Neuroptera Planipennia. Sprawozdania. Komisji Fizyograficzna, 45: 39-44.

Eglin, W., 1939. Zur Biologie und Morphologie der Raphidien und Myrmeleoniden (Neuropteroidea) von Basel und Umgebung. Verhandlungen der Naturforschende Gesellschaft in Basel, 50: 163-220.

Eglin, W., 1940. Die Neuropteren der Umgebung von Basel. Revue suisse de Zoologie, 47: 243-358.

Eglin, W., 1941. Beitrag zur Kenntnis der Neuropteroidea des Wallis. Bulletin de la Murithienne, 58: 63-95.

Eglin-Dederding, W., 1967. Sialis nigripes PICTET, Raphidia (Agulla) aloysiana COSTA und Inocellia keiseri ASPÖCK, neu für die Schweiz. Mitteilungen der schweizerischen entomologischen Gesellschaft, 39: 205-206.

Eglin-Dederding, W., 1968. Versuche einer zoogeographischen Beurteilung der Schweizer Neuropteren. Mitteilungen der schweizerischen entomologischen Gesellschaft, 41: 359-362.

Eglin-Dederding, W., 1979. Die Netzflügler der Schweiz und ihre regionale Verteilung (Insecta, Neuropteroidea). Entomologica Basiliensia, 4: 491-497.

Eglin-Dederding, W., 1980a. Die Netzflügler des Schweizerischen Nationalparks und seiner Umgebung (Insecta: Neuropteroidea). Ergebnisse der wissenschaftlichen Untersuchungen im schweizerischen Nationalpark, 15: 281-351.

Eglin-Dederding, W., 1980b. Die Insektenfauna des Hochmoores Balmoos bei Hasle, Kanton Luzern. III Neuropteroidea und Mecoptera (Netzflügler und Schnabelfliegen). Entomlogische Berichte, 3: 76-85.

Eglin-Dederding, W., 1980c. Auf Netzflüglerfang in den Gebirgswäldern des Schweizerischen Nationalparks (Insecta, Neuropteroidea), 1938-78. Práce Muzea v Hradci Králové, Série A, Vedy Prírodní. Suppl., 1980: 31-34.

Eglin-Dederding, W., 1984. Probleme beim Netzflüglerfang in den Gebirgswäldern des Schweizerischen Nationalparks 1938-1978 (Insecta, Neuropteroidea). Entomologische Gesellschaft Basel, 34(2): 54-57.

Eglin-Dederding, W., 1986. Ökologische Untersuchungen im Unterengadin: Netzflügler und Schnabelfliegen (Neuropteroidea, Mecoptera). Ergebnisse der wissenschaftlichen Untersuchungen im Schweizerischen Nationalparks, 12: 169-200.

Engel, M. S., 1995. A new fossil snake-fly species from Baltic amber (Raphidioptera: Inocelliidae). Psyche, 102: 187-193. http://dx.doi.org/10.1155/1995/23626

Engel, M. S., 2002. The smallest snakefly (Raphidioptera: Mesoraphidiidae): a new species in Cretaceous amber from Myanmar, with a catalog of fossil snakeflies. American Museum Novitates, 3363: 1-22. http://dx.doi. org/10.1206/0003-0082(2002)363\%3C0001:TSSRMA\%3E2.0.CO;2

Engel, M. S., 2003. A new Eocene-Oligocene snakefly from Florissant, Colorado (Raphidioptera: Raphidiidae). Transactions of the Kansas Academy of Sciences, 106: 124-128. http://dx.doi.org/10.1660/00228443(2003)106\%5B0124:ANESFF\%5D2.0.CO;2

Engel, M. S., 2011. A new snakefly from the Eocene Green River Formation (Raphidioptera: Raphidiidae).
Transactions of the Kansas Academy of Sciences, 114: 77-87. http://dx.doi.org/10.1660/062.114.0107

Engel, M. S. \& Grimaldi, D. A., 2008. Diverse Neuropterida in Cretaceous amber, with particular reference to the paleofauna of Myanmar (Insecta). Nova Supplementa Entomologica, 20: 1-86.

Erichson, W. F., 1839. Beiträge zu einer Monographie von Mantispa, mit einleitenden Betrachtungen über die Ordnungen der Orthopteren un Neuropteren. Zeitschrift für die Entomologie (Germar), 1: 147-173.

Erichson, W. F., 1844. Bericht über die wissenschaftlichen Leistungen in der Naturgeschichte der Insecten, Arachniden, Crustaceen u. Entomostraceen während des Jahres 1843. Archiv für Naturgeschichte, 10(2): 249-346.

Erichson, W. F., 1846. Bericht über die wissenschaftlichen Leistungen in der Naturgeschichte der Insecten, Arachniden, Crustaceen und Entomotraceen während des Jahres 1845. Archiv für Naturgeschichte, 12(2): 185-316.

Esben-Petersen, P., 1906. Neuroptera Danica. Entomologiske Meddelelser, 1906-1910(8): 21-49.

Esben-Petersen, P., 1913a. Megaloptera: Fam. Raphididae. Genera Insectorum, 154: 1-12.

Esben-Petersen, P., 1913b. Addition to the knowledge of the Neuropterous insect fauna of Corsica. 2. Entomologiske Meddelelser, 10: 25-28.

Esben-Petersen, P., 1914. Bestemmelsestabel over Danmarks Mecopter, Megalopter og Neuropter. Flora og Fauna, 1914: 129-144.

Esben-Petersen, P., 1929. Netvinger og Skorpionfluer. Danmarks Fauna, 33: 1-134.

Esben-Petersen, P., 1931. Inventa entomologica itineris Hispanici et Maroccani, quod a.1926 fecerunt Harald et Håkan Lindberg 8. Neuroptera. Commentationes biologicae, 3: 1-6.

Esben-Petersen, P., 1939. Bidrag til en Fortegnelse over Bornholms Insektfauna. 5. Supplement. Flora og Fauna, 45: 55-59.

Esben-Petersen, P., 1940. Bidrag til Fortegnelse over Sønderjyllands Insektenfauna. 4. Sonderjyllands Neuropter og Mecopterer (Netvinger og Skorpionsfluer). Flora og Fauna, 46: 1-8.

Fabricius, J. C., 1775. Systema entomologiae, sistens insectorum classe, ordines, genera, species, adiectis synonymis, locis, descriptionibus obervationibus. Flensbvrgi et Lipsiae. 832 pp.

Fabricius, J. C., 1777. Genera Insectorum eorumque characteres naturales secundum numerum, figuram, situm et proportionem omnium partium oris adiecta Mantissa specierum nuper detectarum. M.F. Bartsch. Chilonii. 310 pp.

Fabricius, J. C., 1781. Species insectorum. Tom. 1. C.E. Bohnii. Hamburgi et Kilonii. 552 pp.

Fabricius, J. C., 1787. Mantissa insectorum sistens eorum species nuper detectas adiectis characteribus genericis, differentiis specidicis, emedationibus, observationibus. Tom. 1. Chr. G. Proft. Hafniae. 348 pp.

Fabricius, J. C., 1793. Entomologia Systematica Emendata et aucta secundum classes, ordines, genera species 
adjectis synonimis, locis obervationibus, descriptionibus. Tom. II. C.G. Proft. Hafniae. 519 pp.

Fernández, J., 2007. Noticia de nuevos taxones para la ciencia en el ámbito íbero-balear y macaronésico. Graellsia, 63(2): 371-403. http://dx.doi.org/10.3989/graellsia.2007.v63.i2.143

Ferris, G. F. \& Pennebaker, P., 1939. The morphology of Agulla adnixa (Hagen) (Neuroptera: Raphidiidae). Microentomology, 4: 121-142.

Fischer, H., 1851. Beiträge zur Insekten-Fauna um Freiburg im Breisgau (Zweite Fortsetzung.) Orthoptera. Schluss. Neuroptera. Jahresbericht des Mannheimer Vereins für Naturkunde 17: 60-70.

Fischer, H., 1966. Die Tierwelt Schwabens. 16. Teil: Die Netzflügler (Neuroptera). Bericht der Naturforschenden Gesellschaft, 18: 150-158.

Fraser, F.C., 1940a. Raphidia maculicollis STEPHENS pupating underground. The Entomologist, 73: 180.

Fraser, F.C., 1940b. Notes and Observations. The Entomologist, 73: 166.

Fraser, F.C., 1951. The Entomological fauna of the the New Forest II. Order Neuroptera Suborder Megaloptera Fam. Raphidiidae. Journal of the Society for British Entomology, 3: 225-235.

Fraser, F. C., 1959. Mecoptera, Megaloptera and Neuroptera. Handbooks for the identification of British insects, 1(12-13): 1-40.

Friedrich, H., 1953. Neuroptera. In: Dr. H.G. Bronns Klassen und Ordnungen des Tierreichs 5. Bd.: Arthropoda 3. Abt.: Insecta 12. Buch, Teil a. Geest \& Portig. Leipzig. 148 pp.

Fuente y Morales, J.M., de la, 1929. [Sin título.] Establecimiento tipográfico del Hospicio Provincial. Ciudad Real. 250 pp.

Gadeau de Kerville, H., 1933. Catalogue des Névroptères, Megaloptères, Rhaphidioptères, Mécoptères, Psocoptères, Plécoptères, Éphéméroptères et Trichoptères de la Normandie. Bulletin de la Société des Sciences Naturelles de Rouen, 66-67: 349-401.

Geijskes, D. C., 1972. Over de Neuroptera-Fauna van Meijendel. Zoologische Bijdragen, 13: 43-58.

Genay, A., 1953. Contribution à l'étude des Névroptères de Bourgogne. Travaux du Laboratoire de zoologie et de la Station aquicole Grimaldi de la Faculté des sciences de Dijon, 3: 1-30.

Geoffroy, É. L., 1762. Histoire abregée des insectes qui se trouvent aux environs de Paris; dans laquelle ces animaux sont rangés suivant un ordre méthodique. Tome. 2. Durand. Paris. 690 pp.

Geoffroy, M., 1799. Histoire abrégée des insects, dans laquelle ces animaux sont rangés suivant un ordre méthodique. Tome II. Delalain. Paris. 744 pp.

Gepp, J., 1973. Vergleichend - Quantitative Untersuchungen der Dichten von Neuropterenimagines in den Jahren 1964 bis 1972 im Kaiserwald südwestlich von Graz. Berichte der Arbeitsgemeinschaft für Ökologische Entomologie in Graz, 1: 29-41.

Gepp, J., 1974. Die Netzflügler (Megaloptera, Raphidiodea, Planipennia) des Kaiserwaldes südwestlich von
Graz (mit einer zoogeographischen Analyse). Mitteilungen der Abteilung für Zoologie am Landesmuseum Joanneum in Graz, 3: 11-28.

Gepp, J., 1975. Die Neuropteren von Graz: Ein Beitrag zur Kenntnis der mitteleuropäischen Grosstadtfauna. Mitteilungen des Naturwissenschaftlichen Vereins für Steiermark, 105: 265-278.

Gepp, J., 1978. Die Raphidiopteren der Steirmark in faunistisch - ökologischer Betrachtung (Neuropteroidea, Insecta). Mitteilungen des Naturwissenschaftlichen Vereins für Steiermark, 108: 241-250.

Gepp, J., 1981. Die Neuropteren der Steiermark. Artenliste, Zusammenstellung steirischer Literaturhinweise sowie neue Fundmeldungen (Neuropteroidea, Insecta). Mitteilungen des Naturwissenschaftlichen Vereins für Steiermark, 11: 193-203.

Gepp, J., 1984. Erforschungsstand der Neuropteren. Larven der Erde (mit einem Schlüssel zur Larvaldiagnose der Familien, einer Übersicht von 340 beschriebenen Larven und 600 Literaturzitaten). In: J. Gepp, H. Aspöck \& H. Hölzel (eds.). Progress in World's Neuropterology (Proceedings of the 1st International Symposium on Neuropterology, Graz, 1980). Graz: 183-239.

Gepp, J., 1986a. Biology and larval diagnosis of Central European Neuroptera (A review of the present knowledge). In: J. Gepp, H. Aspöck \& H. Hölzel (eds.). Recent Research in Neuropterology (Proceedings of the 2nd International Symposium on Neuropterology, Hamburg, 1984). Graz: 137-144.

Gepp, J., 1986b. Die Neuropteren Liechtensteins. Eine Faunistische Übersicht. Naturkundliche Forschung im Fürstentum Liechtenstein, 6: 1-28.

Girard, M., 1864. Considérations générales sur le genre Raphidia (Névroptères, Raphidiens) et note sur les espèces de ce genre qui se trouvent aux environs de Paris. Annales de la Société Entomologique de France, 4: 669-675.

Girard, M., 1879. Raphidides. In: Traité élémentaire d'entomologie. Tome II. J. B. Baillière \& fils. Paris: 492-506.

Goldfuss, G. A., 1820. Handbuch der Zoologie. I. Abt. J. L. Schrag. Nürnberg. 696 pp.

Grassé, P. P., 1951. Ordre des Mécoptères. In: P. P. Grassé (ed.). Traité de Zoologie, t. 10, fasc. 1. Masson et Cie. Paris: 71-124.

Greve, L., 1987. Bestemmelsestabell for Norske Nettvinger (Planipennia), Kamelhalsfluer (Raphidioptera) og Mudderfluer (Megaloptera). Norske Insekttabeller, 12: 1-43.

Greve, L., 2002. The order Raphidioptera in Norway. Norwegian Journal of Entomology, 49: 81-92.

Grimaldi, D. A., 2000. A diverse fauna of Neuropterodea in amber from the Cretaceous of New Jersey. In: D. Grimaldi (ed.). Studies on fossils in amber, with particular reference to the Cretaceous of New Jersey. Backhuys Publishers. Leiden: 259-303.

Grimaldi, D. A. \& Engel, M. S., 2005. Evolution of the Insects. Cambridge University Press. New York. xv+755 pp.

Grimaldi, D. A., Engel, M. S. \& Nascimbene, P. C., 2002. Fossiliferous Cretaceous amber from Myanmar (Burma): its rediscovery, biotic diversity, and peleontological 
significance. American Museum Novitates, 3361: 1-72. http://dx.doi.org/10.1206/0003-0082(2002)361\%3C0001:FCAFMB\%3E2.0.CO;2

Gruppe, A., 1997. Beitrag zur Kenntnis der Raphidiopteren-Fauna Oberbayerns (Neuropteroidea). Nachrichtenblatt der Bayerischen Entomologen, 46(1/2): 26-28.

Gruppe, A., Grossner, M. \& Simon, U., 2004. Neuropteren in Wäldern Schwabens und Oberbayerns (Insecta: Neuropteroidea). Beitäge zur bayerischen Entomofaunistik, 6: 243-254.

Gruppe, A. \& Schubert, H., 2001. The spatial distribution and plant specificity of Neuropterida in different forest sites in Southern Germany. Beiträge zur Entomologie, 51(2): 517-527.

Guérin-Méneville, F. E., 1845. Sur la dépouille d' un nymphe de Raphidie. Annales de la Société Entomologique de France, 2(3): 34.

Günther, K., 1968. Ordnung Raphidioptera, Kamelhalsfliegen. In: Urania Tierreich, Insekten. Urania-Verlag. Leipzig, Jena und Berlin: 399-402.

Günther, K., 1994. Ordnung Raphidioptera - Kamelhalsfliegen. In: Urania Tierreich in sechs Bänden. Insekten. Urania-Verlag. Leipzig, Jena und Berlin: 221-225.

Gustafson, J.F., 1950. The Origin and Evolution of the Genitalia of the Insecta. Microentomology, 15(2): 35-67.

Hagen, H.A., 1844. Die neueren Arbeiten über das Genus Raphidia Linn. Stettiner Entomologische Zeitung, 5: 180-186.

Hagen, H.A., 1850. Übersicht der neuern Literatur, betreffend die Neuroptera Linné (Fortsetzung). Stettiner Entomologische Zeitung, 11: 362-378.

Hagen, H.A., 1854. Die Kupfer zu Scopoli’s Entomologia Carnicola. Stettiner Entomologische Zeitung, 15: 81-91.

Hagen, H.A., 1858. Synopsis of the British Planipennes. Entomologist's Annual, 1858: 17-33.

Hagen, H.A., 1860. Über die Neuropteren-Fauna Amerikas. Stettiner Entomologische Zeitung, 21: 209-216.

Hagen, H. A., 1861. Synopsis of the Neuroptera of North America. With a list of the South American species. Smithsonian Miscellaneous Collections, 4: 1-347. http:// dx.doi.org/10.5962/bhl.title.60275

Hagen, H. A., 1864. Névroptères (non Odonates) de la Corse, recueillis par M.E. Bellier de la Chavigneri en 1860 et 1861. Annales de la Société Entomologique de France, 4: 38-45.

Hagen, H. A., 1866. Synopsis des Névroptères d'Espagne. Section II. Neuroptera. Nèvroptères a Metamorphoses complètes. H.G. Baillière \& F. Savy, Paris, et H. Georg, Genève. 123 pp.

Hagen, H. A., 1867. Notes on the Genus Raphidia. Transactions of the Entomological Society of London, 5: 493-499.

Haines, F. H., 1922. Neuroptera in Dorset. The Entomologist, 55: 84-85.

Handlirsch, A., 1903. Zur Phylogenie der Hexapoden (Vorläufige Mitteilung). Sitzungsberichte der Akademie der Wissenschaften in Wien, 112: 716-738.

Handlirsch, A., 1904. Zur Systematik der Hexapoden. Zoologischer Anzeiger, 27: 733-759.
Handlirsch, A., 1906-1908. Die fossilen Insekten und die Phylogenie der rezenten Formen. W. Engelmann. Leipzig. 1430 pp.

Handlirsch, A., 1913. Insekten. In: Paläontologie. Handwörterbuch Naturwissenschaftliche, 2 Aufläge. Bande V: 793-822.

Handlirsch, A., 1925. Geschichte, Literatur, Technik, Paläontologie, Phylogenie, Systematik. In: C. Schröder (ed.). Handbuch der Entomologie, vol. 3. G. Fischer. Jena: 1-1201.

Handlirsch, A. \& Beier, M., 1936. Ordnung der Pterygogenea: Raphidides=Kamelhalsfliegen. Handbuch der Zoologie, 4(2): 1402-1413.

Haring, E., Aspöck, H., Bartel, D. \& Aspöck, U., 2011. Molecular phylogeny of the Raphidiidae (Raphidioptera). Systematic Entomology, 36(1): 16-30. http://dx. doi.org/10.1111/j.1365-3113.2010.00542.x

Hellen, W., 1922. Nya fyndlokaler för Neuroptera. Notulae Entomologicae, 2(1): 24-25.

Hennig, W., 1953. Kritische Bemerkungen zum phylogenetischen System der Insekten. Beiträge zur Entomologie, Sonderhefte, 3: 1-85.

Hennig, W., 1969. Die Stammesgeschichte der Insekten. W. Kramer Verlag. Frankfurt a.M. 436 pp.

Hennig, W., 1981. Insect Phylogeny. John Wiley \& Sons. Chichester, New York, Brisbane \& Toronto. 514 pp.

Henry, C. S., 1982. Neuroptera. In: S. P. Parker (ed.). Synopsis and classification of living organisms, vol. 2. McGraw - Hill. New York: 470-482.

Henry, L. M., 1958. Musculature of the cervical region in insects. Microentomology, 23: 95-105.

Heyden, L. V., 1896. Die Neuroptera-Fauna der weiteren Umgebung von Frankfurt a.M. Bericht der Senckenbergischen Naturforschenden Gesellschaft in Frankfurt am Main, 1896: 105-123.

Hinton, H. E., 1947. A new classification of insect pupae. Proceedings of the Zoological Society of London, 116: 282-328. http://dx.doi.org/10.1111/j.1096-3642.1946. tb00124.x

Hoffmann, N. J., 1962. Faune des Nevroptérö̈des du Grand-Duché de Luxembourg. Archives du Institut Grand-ducal de Luxembourg, 28: 249-332.

Hollier, J. A. \& Belshaw, R. D., 1993. Stratification and phenology of a woodland Neuroptera assemblage. The Entomologist, 112 (3/4): 169-175.

Hölzel, H., 1964. Die Netzflügler Kärntens. Carinthia, 2(74): 97-156.

Hölzel, H., 1973. Die Netzflügler Kärntens. 1. Nachtrag. Carinthia, 2(83): 497-506.

Hölzel, H., Aspöck, H. \& Aspöck, U., 1980. Eine systematisches Verzeichnis aller auf östereichischem Gebiet festgestellten Tierarten. Neuropteroidea. Catalogus faunae Austriae, 17: 1-26.

Hölzel, H. \& Wieser, C., 1999. Die Netzflügler Kärntens. Eine zusammenfassende Darstellung der Autökologie und Chorologie der Neuropterida (Megaloptera, Raphidioptera, Neuroptera) Kärntens. Carinthia, 2(189/109): 361-429. 
Hong, Y. C., 1992. The study of early Cretaceous Coleoptera, Raphidioptera, Diptera (Insecta) of Kezuo, West Liaoning Province. Acta Geologica Gansu, 1: 1-15.

Hong, Y. C. \& Chang, J. P., 1989. A new family "Jilinoraphidiidae" of Raphidioptera (Insecta). Geoscience, 1989: 290-297.

Horstmann, K., 1973. Revision der westpaläarktischen Arten der Gattung Nemeritis Holmgren (Hymenoptera, Ichneumonidae). Opuscula Zoologica, 125: 1-14.

Horstmann, K., 1975. Neubearbeitung der Gattung Nemeritis Holmgren (Hymenoptera, Ichneumonidae). Polskie Pismo Entomologiczne, 45: 251-265.

Horstmann, K., 1976. Wenig bekannte oder neue europäische Hemitelinen- Gattungen (Hymenoptera, Ichneumonidae, Cryptinae). Nachrichtenblatt der Bayerischen Entomologen, 25: 22-31.

Horstmann, K., 1994. Nachtrag zur Revision der westpaläarktischen Nemeritis-Arten (Hymenoptera, Ichneumonidae, Campopleginae). Mitteilungen der Münchner Entomologischen Gesellschaft, 84: 79-90.

Hufnagel, M., 1987. Fossile Käferfaunen in typischenn Quarteprofilen aus Schleswig-Holstein und ihre Bedeutung als Klimaindikatoren. Berichte Reports, Geologisch-Paläontologisches Institut und Museum der Universität Kiel, 18: 1-144.

Jahn, E., 1942. Untersuchungen über die Artrophodenfauna von Tannenbeständen und ihre Emfindlichkeit gegen Insektizide. Centralblatt für das gesamte Forstwesen, 68: 151-171.

Jander, U., 1966. Untersuchungen zur Stammesgeschichte der Putzbewegungen von Tracheaten. Zeitschrift für Tierpsychologie, 23: 799-844.

Jepson, J. E., Coram, R. A. \& Jarzembowski, E. A., 2009. Raphidioptera (Insecta: Neuropterida) from the Lower Cretaceous Purbeck Limestone Group, Dorset, U.K. Cretaceous Research, 30: 527-532. http://dx.doi. org/10.1016/j.cretres.2008.09.006

Jepson, J. E. \& Jarzembowski, E. A., 2008. Two new species of snakefly (Insecta: Raphidioptera) from the Lower Cretaceous of England and Spain with a review of other fossil raphidiopterans from the Jurassic/Cretaceous transition. Alavesia, 2: 193-201.

Jepson, J. E., Makarkin, V. N. \& Coram, R. A., 2012. Lacewings (Insecta: Neuroptera) from the Lower Cretaceous Purbeck Limestone Group of southern England. Cretaceous Research, 34: 31-47. http://dx.doi. org/10.1016/j.cretres.2011.10.001

Jong, C., 1978. Entomologische Notities: Gegevens over Nederlandse Neuroptera 3. Entomologische Berichten, 38: 4.

Joost, W., 1973. Neuropteren aus Bulgarien. Entomologische Nachrichten Fresden, 17: 145-156.

Kacirek, A., 1994. Bemerkungen zum Vorkommen der Neuroptera und der Raphidioptera auf dem Gebiet des Adlergebirges und Unteradlergebierges. Panorama Natura, 2: 67-71.

Karl, O., 1937. Ein Beitrag zur Kenntnis der Insektenfauna Ostpommerns. Dohrniana, 16: 56-61.

Kästner, A., 1934. Zur Lebensweise der Kamelhalsfliegen (Raphidiina). Zoologischer Anzeiger, 108: 1-11.
Kästner, A., 1973. Lehrbuch der Speziellen Zoologie. Bd. 1, Wirbellose, 3. Teil (Insecta: B. Spez. Teil). G. Fischer Verlag. Stuttgart: 519-533.

Kéler, S., 1963. Entomologisches Wörterbuch mit besonderer Berücksichtigung der morphologischen Terminologie. Akademie-Verlag. Berlin. 774 pp.

Kevan, D. K., 1979. 38. Raphidioptera. In: H. V. Danks (ed.). Canada and its insects fauna. Memoirs of the Entomological Society of Canada, 108: 352-354.

Kielhorn, K. H., 1991. Fluginsekten im Kronenraum von Kiefern und Eichen. Diplomarbeit. Institut für Angewandte Zoologie und Tierphysiologie, Bodenzoologie und Öcologie, Freie Universität. Berlin. 90 pp.

Killington, F. J., 1926. Notes on Neuroptera taken in 1925. The Entomologist, 59: 110-112.

Killington, F. J., 1927. Notes on the orders Odonata, Neuroptera and Mecoptera of Hamphshire. Transactions of the Hampshire Entomological Society and the South of England, 3: 37-42.

Killington, F. J., 1928. Notes on the Neuroptera and Mecoptera of Hampshire. Transactions of the Hampshire Entomological Society and the South of England, 4: 49-52.

Killington, F. J., 1929. A synopsis of British Neuroptera. Transactions of the Entomological Society of Hampshire and the South of England, 5: 1-36.

Killington, F. J., 1931. Notes on British Neuroptera. The Entomologist, 64: 73-76.

Killington, F. J., 1932a. Neuroptera Planipennia and Megaloptera from the Oxford District. Journal of the Entomological Society of the South of England, 1(1): 8-10.

Killington, F. J., 1932b. A contribution towards a biological survey of the Megaloptera and Neuroptera of Hampshire and the Isle of Wight. Transactions of the Entomological Society of Hampshire and the South of England, 7: 56-67.

Kimmins, D. E., 1930. A List of the Corsian Ephemeroptera and Neuroptera. Eos, 6: 185-190.

Kimmins, D. E., 1933. Some records of Odonata, Ephemeroptera, Plecoptera, Neuroptera and Trichoptera in Devonshire. Journal of the Entomological Society of the South of England, 1(4): 86-91.

Kimmins, D. E., 1934. A new Species of Coniopteryx (Neuroptera) from France. Annals and Magazine of Natural History, 10(13): 613-619.

Kimmins, D. E., 1939. Neuroptera, Megaloptera and Mecoptera from Kent and Sussex. Journal of the Society for British Entomology, 2(1): 12.

Kimmins, D. E., 1970. A list of the type-specimens of Plecoptera and Megaloptera in the British Museum (Natural History). Bulletin of the British Museum of Natural History, Entomology, 24: 335-361.

Kis, B., 1959. Faunenkatalog der bisher in der Rumänischen Volksrepublik bekannten Neuropteren und Mecopteren. Folia entomologica hungarica, 12: 331-347.

Kis, B., 1984. Lordre Raphidioptera (Insecta) en Roumanie. Travaux du Muséum d'Histoire Naturelle "Grigore Antipa”, 26: 85-97. 
Kis, B. \& Stamp, H. M., 1964. Katalog der Neuropterensammlung des Brukenthalmuseums in Sibiu (Hermannstadt). Entomologische Abhandlungen, 32: 53-60.

Klapálek, F., 1894. Descriptions of a new species of Raphidia, L. and of three new species of Trichoptera from the Balkan Peninsula, with critical remarks on Panorpa gibberosa, McLach. Transactions of the Entomological Society of London, 42: 489-495.

Klapálek, F., 1895. Verzeichnis der Insekten Böhmen’s herausgegeben von der Gesellschaft für Physiokratie in Böhmen. Catalogus insectorum faunae bohemicae, 4: $1-20$.

Klapálek, F., 1903a. Zpráva o výzkumu ceských Neuropteroid v.r. 1902. Věstnik České Akademie Císare Františka Josefa pro Vedy, Slovesnost a Umení, 12: 257-264.

Klapálek, F., 1903b. Über neue und wenig bekannte Arten der paläarktischen Neuropteroiden. Bulletin International de l'Académie des Sciences de Bohême, 7: 1-14.

Klapálek, F., 1906. Př́spěvek ke znalosti fauny Neuropteroid Chorvatska, Slavonska i zemí sousedních. Věstník České Akademie Císare Františka Josefa pro Vedy, Slovesnost a Umení, 15(16): 1-18.

Klefbeck, E. \& Tjeder, B., 1946. Insekter från södra Bohuslän. Entomologisk Tidskrift, 67: 198-209.

Kleinsteuber, E., 1972. Beitrag zu einer Netzflüglerfauna Sachsens. Veröffentlichungen des Museums für Naturkunde Karl-Marx-Stadt, 7: 59-88.

Kleinsteuber, E., 1974. Verzeichnis der im Gebiet der Deutschen Demokratischen Republik bisher festgestellten Neuropteren. Entomologische Nachrichten, 18: 145-153.

Kleinsteuber, E., 1994. Vorläufiges Verzeichnis der Schlammfliegen (Megaloptera), Kamelhalsfliegen (Raphidioptera), Netzflügler (Planipennia) und Schnabelfliegen (Mecoptera) des Freistaates Sachsen. Mitteilungen Sächsischer Entomologen, 27: 17-19.

Klingstedt, H., 1935. Neuroptera. Enumeratio Insectorum Fenniae, 4: 14-15.

Klingstedt, H., 1937. Chromosome Behaviour and Phylogeny in the Neuroptera. Nature, 139: 464-469. http:// dx.doi.org/10.1038/139468a0

Kloet, G. S. \&. Hincks, W. D., 1945. Raphidioidea. In: A check list of British Insects. T. Buncle \& Co. Arbroath. 483 pp.

Kloet, G. S. \& Hincks, W. D., 1964. A check list of British Insects. 2nd Edition. Part I. Royal Entomological Society of London. London. 119 pp.

Kofler, A., 1977. Schlammfliegen und Kamelhalsfliegen aus Osttirol. Carinthia, 287: 337-341.

Kovarik, P. W., Burke, H. R. \& Agnew, C. W., 1991. Development and Behavior of Snakefly, Raphidia bicolor Albarda (Neuroptera: Raphidiidae). Southwestern Entomologist, 16(4): 353-364.

Kowalska, T., 1978. Drapieżne siatkoskrzydłe. In: J. Boczek \& J. J. Lipa (eds.). Biologiczne metody walki ze szkodnikami. PWN. Warszawa: 211-223.

Krause, R. \& Ohm, P., 1970. Zur Neuropterenfauna der Sächsischen Schweiz (1). Staatliches Museum für Tierkunde in Dresden, 4: 25-30.
Krausse, A. \& Wolff, M., 1919. Eine Übersicht über die bisher aufgestellten fossilen und rezenten Insektenordnungen. Archiv für Naturgeschichte, 85: 151-171.

Kristensen, N. P., 1975. The phylogeny of hexapod “orders”. A critical review of recent accounts. Zeitschrift für Zoologische Systematic und Evolutionsforschung, 13: 1-44. http://dx.doi.org/10.1111/j.1439-0469.1975.tb00226.x

Kristensen, N. P., 1981. Phylogeny of Insects orders. Annual Review of Entomology, 26: 135-157. http://dx. doi.org/10.1146/annurev.en.26.010181.001031

Kristensen, N. P., 1991. Phylogeny of extant Hexapods. In: I. D. Naumann (ed.). The Insects of Australia. A textbook for students and research workers. $2^{\text {nd }}$ edition. Vol. 1. Melbourne University Press. Melbourne: 125-140.

Kukalová-Peck, J., 1991. Fossil history and the evolution of Hexapod structures. In: I. D. Naumann (ed.). The Insects of Australia. A textbook for students and research workers. $2^{\text {nd }}$ edition. Vol. 1 . Melbourne University Press. Melbourne: 141-179.

Kusdas, K., 1972. Beitrag zur Kenntnis der Insektenfauna von Korsica. Zeitschrift der Arbeitsgemeinschaft Österreichischer Entomologen, 24(4): 153-166.

Lackschewitz, P., 1922. Die Neuropteren und Trichopteren des Ostbaltischen Gebietes. Archiv für die Naturkunde des Estlands, 14(3): 1-64.

Lackschewitz, P., 1929. Nachtrag zu den Neuropteren und Trichopteren des Ostbaltischen Gebietes. Eesti Loodusteaduse Arhiiv, 2 seeria, 14(3): 1-40.

Lacroix, J. L., 1912. Contribution à l'étude des Névroptères de France (Première [1] liste.). Feuille des Jeunes Naturalistes, 42: 43-49, 53-56.

Lacroix, J. L., 1913. Quelques familles de Névroptères de France. - Diagnoses synoptiques. Mémoires de la Société Historique et Scientifique des Deux-Sèvres, 1913: 1-27.

Lacroix, J. L., 1933. Notes névroptèrologiques 11. Lambillionea, 33: 146-152.

Lakon, G., 1939. Entomophthoraceen - Studien 5-6. Zeitschrift für Angewandte Zoologie, 26: 517-521.

Lamarck, J., 1817. Histoire naturelle des animaux sans vertèbres, présentant les caractères généraux et particuliers de ces animaux, leur distribution, leurs classes, leurs familles, leurs genres, et la citation des principales espèces qui s'y rapportent; précédée d'une introduction offrant la détermination des caractères essentiels de l'animal, sa distinction du végétal et des autres corps naturels, enfin, l'exposition des principes fondamentaux de la zoologie. Tome 4. Deterville \& Verdière. Paris. 603 pp.

Latreille, P. A., 1796. Précis des caractères génériques des Insectes, disposés dans un ordre naturel. F. Bourdeaux. Brive. 198 pp.

Latreille, P. A., 1798. Observations sur la Raphidie ophiopsis. Bulletin de la Société Philomathique de Paris, 2(20): 153-154.

Latreille, P. A., 1802. Histoire naturelle, générale et particulière, des Crustacés et des Insectes. Tom 3. Familles naturelles de genres. F. Dufart. Paris. 967 pp.

Latreille, P. A., 1805. Histoire naturelle, génerale et particulière, des Crustacés et des Insectes. Tom 13. F. Dufart. Paris. 432 pp. 
Latreille, P. A., 1807. Genera Crustaceorum et Insectorum secundum ordinem naturalem in familias disposita, iconibus exemplisque plurimis explicata. Tom. 3. A. Köenig. Paris. 258 pp.

Latreille, P. A., 1810. Considérations générales sur l'ordre naturel des animaux composant les classes des Crustacés, des Arachnides et des Insectes; avec un tableau méthodique de leurs genre disposés en famille. F. Schoell. Paris. 444 pp.

Latreille, P. A., 1825a. Familles naturelles de règne animal, exposées succinctement et dans un ordre analytique, avec lindication de leurs genres. J.-B. Baillière. Paris. $570 \mathrm{pp}$.

Latreille, P. A., 1825b. Encyclopédie méthodique. Histoire naturelle. Entomologie, ou Histoire naturelle des Crustacés, des Arachnides et des Insectes. Vol. 10. Agasse. Paris. 832 pp.

Lauffer, J., 1921. Insectos del Escorial recogidos por D. Jorge Lauffer. Boletín de la Sociedad Entomológica de España, 4: 49.

Lauterbach, K. E., 1972. Beschreibung zweier neuer europäischen Inocelliiden (Insecta, Raphidioptera), zugleich ein Beitrag zur vergleichenden Morphologie und Phylogenie der Kamelhalsfliegen. Bonner Zoologische Beiträge, 23: 219-252.

Lauterbach, K. E., 1974. Sin die Raphidiidae die ursprüngliche Familie der Raphidioptera? Mitteilungen des Internationalen Entomologischen Vereins e.V. Frankfurt a.M. gegr. 1884, 84: 1-7.

Leach, W. E., 1815. Entomology. In: D. Brewster (ed.). The Edinburgh Enciclopaedia, Vol. 9, pt. 1. Edinburgh: 57-172.

Lestage, J. A., 1928. Une Inocellia nouvelle du Maroc et Notes critiques sur les Raphidioidea (Megaloptera). Bulletin de la Société des Sciences Naturelles et Physiques du Maroc, 8: 26-33.

Letardi, A., 1998. Present knowledge of Italian Neuropterida: history, distribution and current problems. Acta Zoologica Fennica, 209: 145-149.

Letardi, A. \& Maltzeff, p., 2003. Neuroptera and Mecoptera of the Tenuta Presidenziale di Castelporziano and of the surrounding area (Neuroptera, Raphidioptera, Mecoptera). Bolletino dellassociazione romana di entomologia, 56(1-4): 49-62.

Letardi, A. \& Pantaleoni, R. A., 1996. I Neurotteroidei W-Paleartici della collezione del Museo di Zoologia dell'Università di Roma (Neuropteroidea). Fragmenta entomologica, 28: 277-305.

Letardi, A. \& Pantaleoni, R.A., 2007. Survey towards an inventory of Italian Neuropterida. Annali del Museo Civico di Storia Naturale di Ferrara, [2005], 8: 87-93.

Linnaeus, C., 1735. Systema natura, sive regna tria naturae, systematica proposita, per classes, ordines, genera et species. Theodorum Haak. Lugduni Batovorum.

Linnaeus, C., 1758. Systema Naturae per Regna tria Naturae, secundum classes, ordines, genera, species, cum characteribus, differentiis, synonymis, locis, Tomus I. Editio 10. Laurentii Salvii. Holmiae. 824 pp.

Linnaeus, C., 1789. Entomologia, Faunae Suecicae Descriptionibus Aucta, Vol. 3. Lugduni. 608 pp.
Linnaeus, C., 1790. Systema Naturae per Regna tria Naturae. Editio 13. Tomus I. Pars V. pp. 2638-2648.

Liu, X., Aspöck, H., Yang, D. \& Aspöck, U., 2010. Species of the Inocellia fulvostigmata group (Raphidioptera, Inocelliidae) from China. Deutsche Entomologische Zeitschrift, 57(2): 223-232. http://dx.doi.org/10.1002/ mmnd.201000019

Liu, X., Aspöck, H., Zhan, C. \& Aspöck, U., 2012. A review of the snakefly genus Sininocellia (Raphidioptera, Inocelliidae): discovery of the first male and description of a new species from China. Deutsche Entomologische Zeitschrift, 59(2): 233-240.

Liu, X., Makarkin, V. N., Yang, Q. \& Ren, D., 2013. A remarkable new genus of basal snakeflies (Insecta: Raphidioptera: Priscaenigmatomorpha) from the Early Cretaceous of China. Cretaceous Research, 45: 306-313. http://dx.doi.org/10.1016/j.cretres.2013. 06.001

Liu, X. Y., Aspöck, H. \& Aspöck, U., 2014a. Inocellia rara sp. nov. (Raphidioptera: Inocelliidae), a new snakefly species from Taiwan, with remarks on systematics and biogeography of the Inocelliidae of the island. Zootaxa, 3753(3), 226-232.

Liu, X. Y., Ren, D. \& Yang, D., 2014b. New transitional fossil snakeflies from China illuminate the early evolution of Raphidioptera. BMC Evolutionary Biology, 14: 84. http://dx.doi.org/10.1186/1471-2148-14-84

Loew, H., 1848. Abbildungen und Bemerkungen zur Anatomie einiger Neuropterengattungen. Linnaea Entomologicam, 3: 345-385.

Lucas, W. J., 1901. Neuroptera, other than Odonata, in 1900. The Entomologist, 34: 70.

Lucas, W. J., 1902. Raphidia larva attacked by a fungus. The Entomologist, 35: 266.

Lucas, W. J., 1909a. Raphidia maculicollis. The Entomologist, 42: 129-130.

Lucas, W. J., 1909b. Raphidia maculicollis (Neuroptera). The Entomologist, 42: 259.

Lucas, W. J., 1909c. A few insects from Braemar. The Entomologist, 42: 282.

Lucas, W. J., 1911. Continental Neuroptera, \& c., taken by Dr. T.A. Chapman in 1909 and 1910. The Entomologist, 44: 96-97.

Lucas, W. J., 1913. British Neuroptera, 1912. The Entomologist, 46: 306-307.

Lucas, W. J., 1914a. British Neuroptera, 1913. The Entomologist, 47: 190-191.

Lucas, W. J., 1914b. Continental insects of various orders taken by Dr. T.A. Chapman in 1913. The Entomologist, 47: 97-99.

Lucas, W. J., 1915. Britisch Neuroptera in 1914. The Entomologist, 48: 179-181.

Lucas, W. J., 1917. Britisch Neuroptera in 1916. The Entomologist, 50: 87-88.

Lucas, W. J., 1920. Notes on Britisch Neuroptera in 1919. The Entomologist, 53: 254-255.

Lucas, W. J., 1922. The South London Entomological Society. The Entomologist, 55: 287. 
Lucas, W. J., 1923. Notes on the British Neuroptera (Incluiding Megaloptera and Mecoptera) in 1922. The Entomogist, 56: 219-221.

Lucas, W. J., 1924. Notes on British Neuroptera (Incluiding Megaloptera and Mecoptera) in 1923. The Entomologist, 57: 177-179.

Lucas, W. J., 1925. Notes on British Neuroptera (Incluiding Megaloptera and Mecoptera) in 1924. The Entomologist, 58: 155-156.

Lucas, W. J., 1926. Notes on the British Neuroptera in 1926. The Entomologist, 59: 289-290.

Lucas, W. J., 1927a. Notes on the British Neuroptera (with Mecoptera) in 1926. The Entomologist, 60: 169-170.

Lucas, W. J., 1927b. A list of the British Neuroptera. The Entomologist, 60: 7.

Lucas, W. J., 1928a. Notes on British Neuroptera and Mecoptera in 1927. The Entomologist, 61: 217-220.

Lucas, W. J., 1928b. Notes on the British Snakeflies (Raphidia). Proceedings of the South London Entomological and Natural History Society, 1928-1929: 34-41.

Lucas, W. J., 1929. Notes on British Neuroptera and Mecoptera: 1928. The Entomologist, 62: 156-159.

Lucas, W. J., 1930. Notes on British Neuroptera and Mecoptera: 1929. The Entomologist, 63: 269-272.

Lucas, W. J., 1931. Notes on British Neuroptera and Mecoptera: 1930. The Entomologist, 64: 243-244.

Lundblad, O., 1925. Über das Vorkommen von Trichobothrien bei Neuropteren und Corrodentien. Entomologisk Tidskrift, 46: 96-101.

Lyle, G. T., 1908. Ova of Raphidia notata (Neuroptera). The Entomologist, 41: 233.

Lyle, G. T., 1913. New Forest Notes, 1912. The Entomologist, 46: 185-188.

Mac Gillavry, D., 1916. De in Nederland voorkomende soorten van het genus Rhaphidia. Entomologische Berichten, 4(88): 254-257.

Mac Gillavry, D., 1949. Een en ander over den levenscyclus can Raphidia (Neur.) en over de naamgeving van deze insecten. Entomologische Berichten, 12(267-294): 116-122.

Mairhuber, F., 1966. Übersicht über die bisher im Bundesland Salzburg aufgefundenen Neuropteren (nebst einigen neuropteren-Funde aus Kärnten und der Steirmark). Nachrichtenblatt der Bayerischen Entomologen, 15: 65-71.

Majewski, E., 1882. Systematyczny spis owadów zylkoskrzydlych Polskich (Insecta Neuroptera Polonica). Warsawa. 42 pp.

Majewski, E., 1885. Owady żyłkoskrzydłe (Neuroptera polonica) systematyczny wykaz krajowych siwciarek $i$ prasiatnic. T. Paprocki. Warszawa. 40 pp.

Makarkin, V. N. \& Archibald, S. B., 2014. A revision of the late Eocene snakeflies (Raphidioptera) of the Florissant Formation, Colorado, with special reference to the wing venation of the Raphidiomorpha. Zootaxa, 3784(4): 401-444. http://dx.doi.org/10.11646/zootaxa.3784.4.4

Makarkin, V. N. \& Khramov, A. V., 2014. A new fossil species of snakeflies (Raphidioptera: Mesoraphidiidae) from the Late Cretaceous of Kazakhstan, with notes on Turonian Neuropterida. Cretaceous Research, 52: 407415. http://dx.doi.org/10.1016/j.cretres.2014.02.010

Malicky, H., Ant, H., Aspöck, H., de Jong, R., Thaler, K. \& Varga, Z., 1983. Argumente zur Existenz und Chorologie mitteleuropäischer (extramediterran-europäischer) Faunen-Elemente. Entomologia Generalis, 9(1/2): 101119. http://dx.doi.org/10.1127/entom.gen/9/1983/101

Mamaev, B. M., Krivošeina, N. P. \& Potockaja, V. A., 1977. Oredelitel litschinok chistshnych nassekomych - entomogagov stvolovych vreditelej. Nauka. Moskva. 392 pp.

Marín, F., 1994. Las comunidades de Neurópteros de la Provincia de Albacete (Insecta: Neuropteroidea). Revista de estudios albacetenses, 34: 247-304.

Marín, F. \& Monserrat, V. J., 1987. Los neurópteros del encinar ibérico. (Insecta, Neuropteroidea). Boletín Sanidad Vegetal. Plagas, 13: 347-359.

Marín, F. \& Monserrat, V. J., 1990. Contribución al conocimiento de los neurópteros de Soria. (Insecta, Neuropteroidea). Boletín de la Asociacion Española de Entomologia, 14: 219-230.

Marín, F. \& Monserrat, V. J., 1991. The community of Neuropteroidea from iberian southern beechwoods. In: L. Polgár, R. J. Chambers, A. F. G. Dixon \& I. Hodek (eds.). Behaviour and impact of Aphidophaga. Academic Publishing. The Hague: 187-198.

Marín, F. \& Monserrat, V. J., 1995. Contribución al conocimiento de los neurópteros de Zaragoza (Insecta, Neuropteroidea). Zapateri, 5: 109-126.

Martin, R., 1893. Les Névroptères Planipennes de L'Indre. Revue d'Entomologie, 12: 142.

Martín Albaladejo, C. \& Izquierdo Moya, I., 2006. Tipos de especies de insectos descritas de la Comunidad de Madrid, conservados en el Museo Nacional de Ciencias Naturales, Inventario preliminar. Graellsia, 62(número extraordinario): 109-144. http://dx.doi.org/10.3989/ graellsia.2006.v62.iExtra.117

Martins-Neto, R. G. \& Nell, N., 1992. Un nouveau fossile de Raphidioptére de la formation Santana, Crétacé inférieur du Brésil (Neuropteroidea, Raphidioptera). Bulletin de la Société Entomologique de France, 97: 425-428.

Martins-Neto, R. G. \& Vulcano, M., 1992. Primeiro registro de Raphidoptera (Neuropteroidea) na Formação Santana (Cretáceo inferior), Bacio do Araripe, nordeste do Brasil. Revista Brasileira de Entomologia, 34: 241-249.

Martynov, A., 1925. To the knowledge of fossil insects from Jurassic beds in Turkestan. Izvestiya Akademii Nauk SSSR, 1925: 233-246.

Martynova, O. M., 1960. Die Kamelhalsfliegen aus dem Perm und Karbon. Verhandlungen XI Internationaler Kongress für Entomology, Wien 1: 302-304.

Martynova, O. M., 1961. Sovremennye i vymeršie verbljudi (Insecta, Raphidioptera). Paleontologicheskii Zhurnal, 3: 73-83.

Martynova, O. M., 1962. Otrjad Raphidioptera. Verbljudki. In: B. B. Rohdendorf (ed.). Osnovy paleontologii. Spravočnik dlja paleontologov i gologov SSSR. Členistonogie, trachejnye i cheicerovye. Izdatel'stvo Akademii Nauk SSSR. Moskva: 270-272. 
Matsuda, R., 1956a. Musculature of the head of Agulla adnixa (Hagen) (Neuroptera, Raphidiidae). Journal of the Kansas Entomological Society, 29: 146-155.

Matsuda, R., 1956b. The comparative morphology of the thorax of two species of insects. Microentomology, 21: 1-65.

Matsuda, R., 1957. Comparative morphology of the abdomen of a Machilid and a Rhaphidiid. Transactions of the American Entomological Society, 83: 39-63.

Matsuda, R., 1976. Morphology and evolution of the insect abdomen. With special reference to developmental patterns and their bearing upon systematics. International series in pure and applied biology, zoology division, vol. 56. Pergamon Press. Oxford. 534 pp.

McLachlan, R., 1868. A Monograph of British Neuroptera-Planipennia. Transactions of the Entomological Society of London, 1868: 145-224. http://dx.doi. org/10.1111/j.1365-2311.1868.tb00622.x

McLachlan, R., 1870. A catalogue of British Neuroptera. Transactions of the Entomological Society of London, 1870: 1-42.

McLachlan, R., 1884. Recherches Névroptérologiques dans les Vosques. Revue d'Entomologie, 1884: 9-20.

McLachlan, R., 1890. Raphidia cognata, Ramb., as a British Insect. The Entomologist's Monthly Magazine, (2), 1(318): 304.

McLachlan, R., 1894. Raphidia notata, F. and R. maculicollis, Steph., common in The New Forest. The Entomologist's Monthly Magazine, 30: 186.

McLachlan, R., 1896. Raphidia Ratzeburgi, Br., on the Simplon. The Entomologist's Monthly Magazine, 32: 42.

McLachlan, R., 1902. An annoted list of Neuroptera- Planipennia collected in Central Spain by Dr. T.A. Chapman and Mr. G.C. Champion in July and August, 1901. The Entomologist's Monthly Magazine, 13: 130-131.

Meinander, M., 1962. The Neuroptera and Mecoptera of eastern Fennoscandia. Fauna Fennica, 13: 1-96.

Meinander, M., 1963. Notes on Finnish Neuroptera and Mecoptera. Notulae Entomologicae, 43: 160-164.

Meinander, M., 1972. A revision of the family Coniopterygidae (Planipennia). Acta Zoologica Fennica, 136: 1-357.

Meinander, M. \& Brander, T., 1963. Über Neuroptera und Mecoptera in Südwest-Häme. Notulae Entomologicae, 43: 82-84.

Metzger, R., 1958. Beiträge zur Morphologie und Biologie einheimischer Kamelhalsfliegen. Dissertation. Potsdam.

Metzger, R., 1960a. Habitat und Verbreitung der Kamelhalsfliegen (Neuroptera, Raphidiidae) in den Waldbeständen östlich und westlich von Berlin. Wissenschaftliche Zeitschrift der Pädagogischen Hochschule, Mathematische-Naturwissenschaftliche Reihe, 5: 199-205.

Metzger, R., 1960b. Die Kamelhalsfliegen (Neuroptera, Raphidiidae). Neue Brehm-Bücherei. Wittenberg. 47 pp.

Metzger, R., 1966. Zur Biologie und Verbreitung der Kamelhalsfliegen (Raphidiidae) und ihrer Larven in Waldbeständen der Mark Brandenburg. Beiträge zur Tierwelt der Mark III. Veröffentlichungen des Bezirksheimatmuseums, 12: 33-42.
Metzger, R., 1967. Zur Stellung der Neuropteren im phylogenetischen System der holometabolen Insekten unter besonderer Berücksichtigung des thorakalen Skeletts ihrer Larven. Wissenschaftliche Zeitschrift der Pädagogischen Hochschule, Mathematische-Naturwissenschaftliche Reihe, 11: 371-378.

Metzger, R., 1969. Zu einigen morphologisch.phylogenetischen Kennzeichen des sternopleuralen Skeletts holometaboler Insektenlarven. Wissenschaftliche Zeitschrift der Pädagogischen Hochschule, MathematischeNaturwissenschaftliche Reihe, 13: 897-909.

Meyer-Dür, L. R., 1875. Die Neuropterenfauna der Schweiz. Mitteilungen der Schweizerischen Entomologischen Gesellschaft, 4: 353-436.

Mickoleit, G., 1965. Über die morphologische Deutung des caudalen Sternocoxalmuskels im Pterothorax der Neuropteroiden. Zoologische Jahrbücher. Abteilung für Anatomie und Ontogenie der Tiere, 82: 521-531.

Mickoleit, G., 1969. Vergleichend-anatomische Untersuchungen an der pterothorakalen Pleurotergalmuskulatur der Neuropteria und Mecopteria (Insecta, Holometabola). Zeitschrift für Morphologie der Tiere, 64: 151-178.

Mickoleit, G., 1973a. Über den Ovipositor der Neuropteroidea und Coleptera und seine phylogenetische Bedeutung (Insecta, Holometabola). Zeitschrift für Morphologie der Tiere, 74: 37-64.

Mickoleit, G., 1973b. Zur Anatomie und Funktion des Raphidiopteren-Ovipositors (Insecta, Neuropteroidea). Zeitschrift für Morphologie der Tiere, 76: 145-171.

Mikulski, J., 1931. Beitrag zur Kenntnis der Fauna des Poprad-Tales in der Gegend von Muszyna (süd-Polen): Ephemeroptera, Trichoptera und Neuroptera. Sprawozdania. Komisji Fizyograficzna, 65: 81-92.

Mjöberg, E., 1909. Nätvingar. Neuroptera. Neuroptera-Planipennia. Svensk Insektenfauna, 8: 130-161.

Mocsary, A., 1918. Pseudo-Neuroptera. Neuroptera. In: Fauna Regni Hungariae Animalium Hungariae, Hucusque Cognitorum Enumeratio Systematica, vol. 3. Arthropoda. Regia Societas Scientiarum Naturalium Hungarica. Budapest: 33-44.

Monserrat, V. J., 1985. Lista de los tipos de Mecoptera y Neuroptera (Insecta) de la colección L. Navás, depositados en el museo de Zoología de Barcelona. Miscellánia Zoológica, 9: 233-243.

Monserrat, V. J., 1986a. Sobre los Neurópteros ibéricos (4) (Neur.). Boletín de la Asociación española de Entomología, 10: 95-105.

Monserrat, V. J., 1986b. Longinos Navás, his neuropterological work and collection. In: J. Gepp, H. Aspöck \& H. Hölzel (eds.). Recent Research in Neuropterology (Proceedings of the 2nd International Symposium on Neuropterology, Hamburg, 1984). Graz: 173-176.

Monserrat, V. J., 1996. Lista de los Neuropteros de Aragón (Megaloptera, Raphidioptera, Planipennia). Catálogo de la Entomofauna Aragonesa, 11: 11-17.

Monserrat, V. J., 2011. Sobre algunas especies de neurópteros de la Península Ibérica y de las Islas Canarias de posición taxonómica problemática o con citas dudosas o cuestionables (Insecta, Neuroptera: Megaloptera, 
Planipennia). Boletín de la Sociedad Entomológica Aragonesa, 49: 153-178.

Monserrat, V. J., 2013. Los Neurópteros (Neuroptera). In: F. Ruano, M. Tierno de Figueroa y A. Tinaut (eds.). Los Insectos de Sierra Nevada. 200 años de historia, Vol. 1. Asociación española de Entomología. Granada: 281-309.

Monserrat, V. J. \& Díaz-Aranda, L. M., 1987. Contribución al conocimiento de los neurópteros (Neur.: Raphidioptera, Planipennia) de Cuenca. Boletín de la Asociación Española de Entomologia, 11: 171-189.

Monserrat, V. J. \& Papenberg, D., 2006. Revisión del género Harraphidia Steinamnn, 1963 con la descripción de dos nuevas especies de la Península Ibérica y de Marruecos (Insecta, Raphidioptera). Graellsia, 62(2): 203-222. http://dx.doi.org/10.3989/graellsia.2006.v62.i2.67

Monserrat, V. J. \& Papenberg, D., 2010. Revisión del género Phaeostigma Navás, 1909 de la Península Ibérica (Insecta, Raphidioptera). Graellsia, 66(1): 47-77. http://dx.doi.org/10.3989/graellsia.2010.v66.010

Monserrat, V. J. \& Papenberg, D., 2012. Revisión del género Venustoraphidia Aspöck \& Aspöck, 1968 de la Península Ibérica (Insecta, Raphidioptera). Graellsia, 68(2): 291-304. http://dx.doi.org/10.3989/graellsia. 2012.v68.061

Monserrat, V. J. \& Triviño, V., 2013. Atlas de los neurópteros de la Península Ibérica e Islas Baleares (Insecta, Neuroptera: Megaloptera, Raphidioptera, Planipennia) / Atlas of the Iberian and Balearic lacewings (Insecta, Neuroptera: Megaloptera, Raphidioptera, Planipennia). Monografías de la Sociedad Entomológica Aragonesa, vol. 13. Zaragoza. 154 pp., 136 mapas.

Morgan, M. J., 1976. Neuroptera and Mecoptera in North Wales: a preliminary survey of their distribution. Entomologist's Gazette, 27: 229-242.

Morley, C., 1909. On the Neuropterous Genus Raphidia, Linn. The Entomologist, 42: 141-143.

Morley, C. \& Elliot, E., 1895. The New Forest in May. The Entomologist's Monthly Magazine, 31: 192-194.

Mosely, M.E., 1932. Corsican Trichoptera and Neuroptera (s.l.) 1931. Eos, 8: 165-185.

Mosely, M.E., 1935. Trichoptera, Plecoptera and Neuroptera, etc., in the Hautes-Pyrénées and the Haute -Garonne. The Entomologist, 68: 205-208.

Müller, G.W., 1912. Der Enddarm emiger Insectenlarven als Bewegungsorgan. Zoologische Jahrbücher. Abteilung für Anatomie und Ontogenie der Tiere, 15: 219-240.

Nagler, C., 1965. Raphidiidae (Neuroptera) semnalate pentru prima datá in Moldova. Revta Muzeelor, 2: 87-88.

Navás, L., 1904. 5. Notas entomológicas [2ª serie]. 11. Neurópteros del Moncayo (Zaragoza). Boletín de la Sociedad Aragonesa de Ciencias Naturales, 3: 139-167.

Navás, L., 1905a. Catálogo descriptivo de los insectos Neurópteros de los alrededores de Madrid. Revista de la Real Academia de Ciencias Exactas, Físicas y Naturales de Madrid, 2, 4: 1-54.

Navás, L., 1905b. Notas entomológicas. XII [b]. Neurópteros de Pozuelo de Calatrava (Ciudad Real). Boletín de la Real Sociedad Española de Historia Natural 5: 502-508.
Navás, L., 1905c. Notas zoológicas. Mis excursiones durante el verano de 1904. Boletín de la Sociedad Aragonesa de Ciencias Naturales, 4: 1-25.

Navás, L., 1905d. Notas neuropterológicas. VI. Neurópteros de Montserrat. Butlletí de la Institució Catalana d'Història Natural, 1(5): 11-21.

Navás, L., 1908. Neurópteros de España y Portugal. Brotéria. Serie Zoológica, 7: 5-131.

Navás, L., 1909a. Neurópteros de los alrededores de Madrid. Revista de la Real Academia de Ciencias Exactas Fisicas y Naturales de Madrid, 8: 370-380.

Navás, L., 1909b. Les Rhaphidies du Musée de Paris. Annales de la Société Scientifique de Bruxelles, 33: 143-146.

Navás, L., 1909c. Catalogue des Raphidiides des collections du Muséum. Bulletin du Muséum National d'Histoire Naturelle, 1(15): 450.

Navás, L., 1910. Mis excursiones entomológicas durante el verano de 1909 (2 julio - 3 Agosto). Butlletí de la Institució Catalana d'Història Natural, 10: 32-56, 74-75.

Navás, L., 1911a. Entomologie Générale, Notes sur quelques Névroptères. Insecta, 1: 239-246.

Navás, L., 1911b. Synopsis de Névroptères de Belgique. Revue Mensuelle de la Société Entomologique Namuroise, 11: 19-22, 27-32, 35-39, 42-44, 51-54, 68-71, 86-90, 97-100, 107-110.

Navás, L., 1912. Notes sur quelques Névroptères. 2 Quelques Névroptères de Corse recueillis par M.G. Bénard. Insecta, 2: 33-36.

Navás, L., 1913a. Cuatro pequeñas colecciones de Neuropteros de la Península Ibérica. Boletín de la Sociedad Aragonesa de Ciencias Naturales, 12: 77-89.

Navás, L., 1913b. Neurópteros del R. Museo Zoológico de Napoles. Annuario del Real Museo Zoologico della Reale Università di Napoli, 4: 1-11.

Navás, L., 1913c. Synopsis de Névroptères de Belgique. Revue Mensuelle de la Société Entomologique Namuroise, 13: 11-13, 26-28, 36-37, 47-50, 57-60, 65-67, 78-80, 90-92, 106-108, 118-120.

Navás, L., 1914a. Algunos Neurópteros de los alrededores de Madrid. Boletín de la Sociedad Aragonesa de Ciencias Naturales, 13: 38-41.

Navás, L., 1914b. Comunicaciones. Notas entomológicas. Neuropteros del Moncayo (Zaragoza). Boletín de la Sociedad Aragonesa de Ciencias Naturales, 13: 207-218.

Navás, L., 1914c. Comunicaciones. Notas entomológicas. Excursión anual de la Sociedad Aragonesa de Ciencias Naturales a Ortigosa y Valvanera (Logroño). Boletín de la Sociedad Aragonesa de Ciencias Naturales, 13: 29-41.

Navás, L., 1915a. Notas sobre Rafídidos (Ins. Neur.). Revista de la Real Academia de Ciencias Exactas Fisicas y Naturales de Madrid, 13: 784-797.

Navás, L., 1915b. Notas sobre Rafídidos (Ins. Neur.). Revista de la Real Academia de Ciencias Exactas Fisicas y Naturales de Madrid, 13: 860-874.

Navás, L., 1915c. Neue Neuropteren. Zweite Serie. Entomologische Mitteilungen, 4: 194-202.

Navás, L., 1915d. Neurópteros nuevos o poco conocidos. Quinta serie. Memorias de la Real Academia de Ciencias y Artes de Barcelona, 11: 455-480. 
Navás, L., 1915e. Neurópteros nuevos o poco conocidos. Cuarta serie. Memorias de la Real Academia de Ciencias y Artes de Barcelona, 11: 373-398.

Navás, L., 1915f. Materiali per una fauna dell' Arcipelago Toscano 9. Algunos Neurópteros de la Isla de Giglio. Annali del Museo Civico di Storia Naturale Giacomo Doria, 6: 276-278.

Navás, L., 1915g. Raphididae Musei Nationalis Hungarici. Annales Historico-Naturales Musei Nationalis Hungarici, 13: 331-336.

Navás, L., 1915h. Particularidades sobre las alas de los insectos. Boletín de la Sociedad Aragonesa de Ciencias Naturales, 14: 108-116.

Navás, L., 1915i. Notes sur les Raphidides (Neur.). Deutsche Entomologische Zeitschrift, Berlin, 1915: 542-544.

Navás, L., 1916. Notas sobre el orden de los Rafidiópteros (Ins.). Memorias de la Real Academia de Ciencias y Artes de Barcelona, 12: 507-513.

Navás, L., 1918a. Neurópteros nuevos o poco conocidos. Memorias de la Real Academia de Ciencias y Artes de Barcelona, 14: 339-366.

Navás, L., 1918b. Excursiones entomológicas por el Norte de la provincia de Lérida. (6-24 de julio de 1917). Butlletí de la Institució Catalana d'Història Natural, 18: 36-49.

Navás, L., 1918c. Rafidiòpter (Ins.) Nou. Arxius de l'Institute d'Estudis Catalans, Seccio de Ciencias, 5: 91-93.

Navás, L., 1919. Monografia de l'Ordre dels Rafidiópters (Ins.). Institute d'Estudis Catalans, Seccio de Ciencias. Barcelona. 90 pp.

Navás, L., 1920. Notas entomológicas: Excursión anual de la Sociedad Aragonesa de Ciencias Naturales al valle de Ordesa (Huesca). (22-29 de Julio de 1918). Boletín de la Sociedad Ibérica de Ciencias Naturales, 19: 37-44.

Navás, L., 1921. Insectos del Escorial recogidos por D. Jorge Lauffer. Boletín de la Sociedad Entomológica de España, 4: 49.

Navás, L., 1923. Sinopsis de los Rafidiópteros (Insectos) de España y Portugal. Asociación Española para el Progreso de las Ciencias, Congreso de Salamanca: 67-73.

Navás, L., 1924. Mis excursiones entomológicas del verano de 1924. Brotéria. Serie Zoológica, 21: 115-150.

Navás, L., 1925. Sinopsis de los Rafidiópteros de Francia. Boletín de la Sociedad Ibérica de Ciencias Naturales, 24: 41-48.

Navás, L., 1927. Insecta Nova. Memorie dell’Accademia Pontificia dei Nuovi Lincei, 10(2): 1-10.

Navás, L., 1928a. Rafidiópteros de la Península Ibérica. Memorias de la Sociedad Entomológica de España, 2: 5-56.

Navás, L., 1928b. Insetti Europei del Museo Civico de Genova. Bollettino della Società Entomologica Italiana, 60: 75-83.

Navás, L., 1928c. Comunicaciones entomológicas. 10. Revista de la Real Academia de Ciencias Exactas Físico-Químicas y Naturales de Zaragoza, 2: 79-134.

Navás, L., 1928d. Insectos del Museo de Hamburgo. Boletín de la Sociedad Entomologica de España, 11: 59-138.
Navás, L., 1931. De mis últimas excursiones entomológicas (1930-1931). Boletín de la Sociedad Entomologica de España, 14: 116-130.

Navás, L., 1933a. La colección de Rafidiópteros (In.) del Museo de Barcelona. Boletín de la Sociedad Entomologica de España, 16: 120-121.

Navás, L., 1933b. Récoltes entomoloqiques en Hollande. Lambillionea, 33: 20-25.

Navás, L., 1934a. Els Rafidiópters del Museu de Ciéncies Naturals de Barcelona. Treballs Museu de Ciencias Naturals de Barcelona, 11(7): 1-7.

Navás, L., 1934b. Fauna de Catalunya. Entomologia de Catalunya. Neuròpters. Fascicle III. Rafidiòpters. Institut d'Estudis Catalans, Secció de Ciències. Barcelona. $67 \mathrm{pp}$.

Navás, L., 1935. Rafidióptero (Ins.) nuevo de España. Boletín de la Sociedad Entomologica de España, 18: 71-74.

Naville, A. \& de Beamont, J., 1936. Recherches sur les Chromosomes des Névroptères. Archives d'Anatomie Microscopique, 32: 271-302.

Nel, A., 1993. Nouveaux Raphidioptères fossiles du Cénozoïque de France et Espagne. EPHE, Biologie et Evolution des Insectes, 6, 1993: 99-108.

Nel, A., Séméria, Y. \& Martins-Neto, R. G., 1990. Un Raphidioptera fossile de Crétacé inférieur du Brésil (Neuropteroidea). Neuroptera International, 6: 27-37.

Newman, E., 1834. Attempted division of British insects into natural orders. Entomological Magazine, London 2: 379-431.

Ocharan, R., Ocharan, F. J. \& Anadón, A., 2012. Neurópteros de la Reserva de la Biosfera de Muniellos (Asturias, Norte de España) (Neuroptera s. s., Megaloptera y Raphidioptera). Boletín de la Sociedad entomológica Aragonesa, 50: 421-429.

Ohm, P., 1961a. Massenauftreten von Boreus und Raphidia (Boreidae/Mecoptera und Raphididae/Neuroptera). Faunistische Mitteilungen aus Norddeutschland, 2: 11-13.

Ohm, P., 1961b. Beziehungen zwischen Körpergrösse und Flügeladerung bei Panorpa (Mecoptera). Sonderdruck aus Zoologischer Anzeiger, 166, 1/2: 1-6.

Ohm, P., 1964. Die Neuropteren und Mecopteren der Umgebung von Plön (Holstein). Faunistische Mitteilungen aus Norddeutschland, 2: 125-128.

Ohm, P. \& Remane, R., 1968. Die Neuropterenfauna Hessens und einiger angrenzender Gebiete (Zur Verbreitung der Neuropteren in Mitteleuropa). Faunistisch-Ökologische Mitteilungen, 3: 209-228.

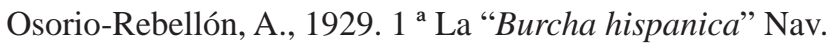
Boletín de la Sociedad Entomologica de España, 12: 35-37.

Oswald, J. D., 1990. Chapter 8. Raphidioptera. In: D. A. Grimaldi (ed.). Insects from the Santana Formation, Lower Cretaceous, of Brazil. Bulletin of the American Museum of Natural History, 195: 154-163.

Oswald, J. D. \& Penny, N. D., 1991. Genus-Group names of the Neuroptera, Megaloptera and Raphidioptera of the world. Ocasional papers of the California Academy of Sciences, 147: 1-94. 
Panov, A. A. \& Davydova, E. D., 1976. Medial Neurosecretory Cells in the Brain of Mecoptera and Neuroptera (Insecta). Zoologischer Anzeiger, 197: 187-206.

Pantaleoni, R. A., 1986. Neurotteri dell'Italia meridionale ed insulare. Animalia, 13: 167-183.

Pantaleoni, R. A., 1988. La Neurotterofauna dell ’Appenino Romagnolo. Atti 15 Congresso Nazionale Italiano di Entomologia, L’Aquila: 633-640.

Pantaleoni, R. A., 1990a. I Neurotteri (Insecta Neuropteroidea) della collezione dell' Istituto di Entomologia Agraria dell'universitá di Padova. Bollettino dell'Istituti di Entomologia "Guido Grandi” della Università degli studi di Bologna, 45: 73-99.

Pantaleoni, R. A., 1990b. Un nuovo ausiliario nel vigneto: Parainocellia bicolor (Costa). Informatore Fitopatologico, 40: 39-43.

Pantaleoni, R. A., 1993. I Neurotteri (Insecta Neuropteroidea) delle collezioni "Zangheri” (Museo di Storia Naturele della Romagna) e "Malmerendi” (Museo Civico di Scienze Naturali di Faenza). Bollettino del Museo Civico di Storia Naturale di Verona, 17: 277-291.

Pantaleoni, R. A., 1994. Neurotteri della Sardegna. Atti 17 Congresso nazionale taliano di Entomologia, Udine: 207-210.

Pantaleoni, R.A., 2005. Interpretation of Achille Costa's data on Neuropterida. Bulletin of Insectology, 58(1): 71-92.

Pantaleoni, R. A., Aspöck, U. \& Cao, O. \& Aspöck, H., 2004. Subilla principae n. sp., A new spectacular snakefly from Sardinia (Raphidioptera Raphidiidae). Redia, 87: 3-6.

Pantaleoni, R. A., Campadelli, G. \& Crudele, G., 1994. Nuovi dati sui Neurotteri dell' alto Appennino romagnolo. Bollettino dell'Istituto di Entomologia "Guido Grandi" della Università degli studi di Bologna, 48: 171-183.

Pantaleoni, R. A. \& Letardi, A., 1998. I Neuropterida della collezione dell'Istituto di Entomologia "Guido Grandi” di Bologna. Bollettino dell'Istituti di Entomologia "Guido Grandi" della Università degli studi di Bologna, 52: 15-45.

Parfitt, E., 1879. The Fauna of Devon. Report and Transactions of the Devonshire Association for the Advancement of Science, 2: 386-421.

Paulus, H. F., 1986a. Comparative morphology of the larval eyes of Neuropteroidea. In: J. Gepp, H. Aspöck \& H. Hölzel (eds.). Recent Research in Neuropterology (Proceedings of the 2nd International Symposium on Neuropterology, Hamburg, 1984). Graz: 157-164.

Paulus, H. F., 1986b. Evolutionswege zum Larvalauge der Insekten - ein Modell für die Entstehung und die Ableitung der ozellären Lateralaugen der Myriapoda von Fazettenaugen. Zoologische Jahrbücher, Abteilung für Systematik, Ökologie und Geographie der Tiere, 113: 353-371.

Penny, N. D., 1981. Neuroptera. In: S. B. Hurlburt, G. Rodriguez \& N. D. dos Santos (eds.). Aguatic Biota of Tropical South America. Part 1 Arthropoda. San Diego State University Press. San Diego: 89-91.

Penny, N. D., Adams, P. H. \& Stange, L. A., 1997. Species catalog of the Neuroptera, Megaloptera, and
Raphidioptera of America and North of Mexico. Proceedings of the California Academy of Sciences, 50: 39-114.

Percheron, M. A., 1833. Mémoire sur les Raphidies. Magazin de Zoologie, d'Anatomie Comparée et de Palaeontologie, 3(66): 1-12.

Pérez de la Fuente, R., Peñalver, E., Delclòs, X. \& Engel, M. S., 2012. Snakefly diversity in Early Cretaceous amber from Spain (Neuropterida, Raphidioptera). ZooKeys, 204: 1-40. http://dx.doi.org/10.3897/zookeys.204.2740

Perkins, J. F., 1956. On the parasite of Raphidia in Britain. The Entomologist, 89-91: 159-160.

Perrichot, V. \& Engel, M. S., 2007. Early Cretaceous snakefly larvae in amber from Lebanon, Myanmar, and France (Raphidioptera). American Museum Novitates, 3598: 1-11. http://dx.doi.org/10.1206/0003-0082(2007)3598\%5B1: ECSLIA\%5D2.0.CO;2

Peters, R. S., Meusemann, K., Petersen, M., Mayer, C., Wilbrandt, J., Ziesmann, T., Donath, A., Kjer, K. M., Aspöck, U., Aspöck, H., Aberer, A., Stamatakis, A., Friedrich, F., Hünefeld, F., Niehuis, O., Beutel, R. G. \& Misof, B., 2014. The evolutionary history of holometabolous insects inferred from transcriptome-based phylogeny and comprehensive morphological data. BMC Evolutionary Biology, 14: 52. http://dx.doi. org/10.1186/1471-2148-14-52

Peterson, A., 1960. Larvae of insects. An introduction to Neartic Species. Part 2. Coleoptera, Diptera, Neuroptera, Siphonaptera, Mecoptera, Trichoptera. Edwards Brothers. Columbus. 416 pp.

Pictet, F. J., 1865. Synopsis des Névroptères d'Espagne. Section 2. Neuroptera. Névroptères a Metamorphoses complètes. H.G. Baillière \& F. Savy, Paris, et H. Georg, Genève. 123 pp.

Plant, C., 1992. A Working List of the Lacewings of the London Area. The London Naturalist, 71: 117-136.

Plant, C., 1994. Provisional atlas of the lacewings and allied insects (Neuroptera, Megaloptera, Raphidioptera and Mecoptera) of Britain and Ireland. In: T. Harding, C. Eversharm \& R. Arnold (eds.). Biological Records Centre. Huntingdon. 203 pp.

Plant, C., 1997. A key to the adults of British Lacewings and their allies (Neuroptera, Megaloptera, Raphidioptera and Mecoptera). Field Studies, 9: 179-269.

Plant, C., 2001. Snake-flies, alderflies and lacewings (Neuropterida) and scorpion flies (Mecoptera): A provisional review of their status and distribution in Essex with notes on their etymology. Essex Naturalist (New Series), 18: 177-218.

Plewka, T., 1974. Bemerkungen über die Neuropterenfauna (Raphidioptera und Planipennia) des Kampinos Nationalparks) (Zentralpolen). Folia Entomologica Hungarica, 27: 287-289.

Poivre, C., 1991. Subilla balesdenti n.sp. (Raphidioptera, Raphidiidae). Neuroptera International, 6: 125-129.

Pongrácz, S., 1919. Beiträge zur Pseudoneuropteren und Neuropterenfauna Polens. Annales Historico-Naturales Musei Nationalis Hungarici, 17: 161-177.

Popov, A., 1964. What do we know on the distribution of Neuropteroidea in Bulgaria. Priroda, 13(6): 68-72. 
Popov, A., 1973. Raphidia ratzeburgi Br. - neu für die Balkaninsel (Raphidioptera). Entomologische Nachrichten, 17: 121-123.

Popov, A., 1974. Eine neue Raphidia aus Tadsikistan (Raphidioptera). Nouvelle Revue d'Entomologie, 4: 153-158.

Popov, A., 1977. 35. Wissenschaftliches Ergebnis der zoologischen Expedition des Nationalmuseums in Prag nach der Türkei. Acta Entomologica Musei Nationalis Pragae, 39: 271-277.

Popov, A., Aspöck, H. \& Aspöck, U., 1978. Raphidia (Xanthostigma) zdravka n.sp. Eine neue Kamelhalsfliege aus dem Kaukasus (Neuropteroidea, Raphidioptera). Zeitschrift der Arbeitsgemeinschaft Österreichischer Entomologen, 30: 17-20.

Porritt, G.T., 1909. The British Raphidiidae. The Entomologist, 42: 183.

Principi, M. M., 1952. Ricerche zoologische sul Massiccio del Pollino (Lucania-Calabria) 6. Neurotteri. Annuario del Museo Zoologico della Reale Università di Napoli, 4: $1-22$.

Principi, M. M., 1960. Contributi allo studio dei Neurotteri Italiani. 15. Descrizione di una nuova specie di Raphidia Linn., R. grandii, (Neuroptera-Raphidiidae) e considerazioni generali sulla morfologia degli ultimi uriti dei Neurotteri. Bollettino dell'Istituto di Entomologia della Reale Università di Bologna, 24: 325-337.

Principi, M. M., 1961. Neurotteri dei Monti Picentini (Appenino Campano). Memorie del Museo Civico di Storia Naturale di Verona, 9: 97-114.

Principi, M. M., 1966. Neurotteri della Basilicata, della Calabria e della Sicilia (Contributi allo studio dei Neurotteri italiani. 18). Memorie del Museo Civico di Storia Naturale di Verona, 14: 363-388.

Principi, M. M., 1980. Neurotteri. In: A. Minelli \& S. Ruffo (eds.). Grande enciclopedia illustrata degli animali. Invertebrati 2. Arnoldo Mondadori. Milano: 105-108.

Pröse, H., 1995. Kommentierte Artenliste der Netzflügler Bayerns (Insecta: Neuropteroidea). Beiträge zur bayerischen Entomofaunistik, 1: 151-158.

Puschnig, R., 1922. Beitrag zur Kenntnis der Netzflügler und Scheinnetzflügler von Kärnten. Carinthia II, 111(31): 58-85.

Puschnig, R., 1923. Weitere Netzflüglerfunde aus Kärnten. Carinthia II, 112-113(32-33): 135-136.

Rambur, M. P., 1842. Histoire Naturelle des Insectes, Névroptères. Librairie encyclopédique de Roret. Fain et Thunot: Paris. [xviii]+534 pp., 12 lám.

Ramme, W., 1911. Entomologische Ergebnisse einer Reise nach Oberitalien und Sütirol (1910) (Neuroptera, Odonata, Orthoptera, Lepidoptera, Diptera, Hymenoptera, Rhynchota). Berliner Entomologische Zeitschrift 56: 11-32.

Ratzeburg, J. T. C., 1844a. Die Forst-Insecten oder Abbildung und Beschreibung der in den Wäldern Preussens und der Nachbarstaaten als schädlich oder nützlich bekannt gewordenen Insecten: in systematischer Folge und mit besonderer Rücksicht auf die Vertilgung der Schädlichen. Dritter Theil. Nicolai. Berlin. viii+314 pp.
Ratzeburg, J. T. C., 1844b. Die Ichneumonen der Forstinsecten in forstlicher und entomologischer Beziehung ein Anhang zur Abbildung und Beschreibung der Forstinsecten. Nicolai. Berlin. 224 pp.

Rausch, H. \& Aspöck, H., 1992. Zur Kenntnis der Larven, der Biologie und Ökologie und der Verbreitung von drei für die südliche Balkan-Halbinsel endemischen Raphidiiden-Spezies (Neuropteroidea: Raphidioptera: Raphidiidae). Zeitschrift der Arbeitsgemeinschaft Österreichischer Entomologen, 44: 35-41.

Rausch, H., Scheibbs, H., Aspöck, H. \& Aspöck, U., 2004. Calabroraphidia renate n. gen., n. sp. eine neue Spezies und ein neues Genus der Familie Raphidiidae aus Süditalien (Neuropterida, Raphidioptera). Entomologische Nachrichten und Berichte, 48: 159-165.

Redtenbacher, J., 1886. Vergleichende Studien über das Flügelgeäder der Insekten. Annalen des K.K. Naturhistorisches Hofmuseums, 1: 153-231.

Remm, H., 1962. Märkmeid Eesti vörktiivaliste (Neuroptera) ja koonuliste (Mecoptera) faunast. The Naturalists Society Attached to the Academy of Sciences of the Estonian SSR, Faunistic Notes. 1: 215-219.

Ren, D., 1994. A new discovery of snake-flies (Insecta) from late Jurassic of Beipiao, Liaoning. Professional Papers of Stratigraphy and Palaeontology, 235: 131-140.

Ren, D., 1995. Systematic Palaeontology. Insecta. In: Faunae and stratigraphy of Jurassic-Cretaceous in Beijing and the adjacent areas. Seismic Publishing House. Beijing: 47-121.

Ren, D., 1997. Studies on the late Mesozoic snake-flies of China (Raphidioptera: Baissopterida, Mesoraphidia, Alloraphidiidae). Acta Zootaxonomica Sinica, 22(2): 172-188.

Ren, D. \& Hong, Y., 1994. A cladistic study on the familial phylogeny of fossil and living Raphidioptera (Insecta). Bulletin of the Chinese Academy of Geological Sciences, 29: 103-108.

Ressl, F., 1964. Abundanzverhältnisse der Neuropteren des Verwaltungsbezirkes Scheibbs, N.Ö. Entomologisches Nachrichtenblatt, 11: 63-68.

Ressl, F., 1969. Über Probleme und Methoden beim Sammeln "seltener" Insekten, die im Larvenstadium häufig in Erscheinung treten. Entomologisches Nachrichtenblatt, 16: 121-123.

Ressl, F., 1983. Naturkunde des Bezirks Scheibbs, Bd. 2. Die Tierwelt des Bezirkes Scheibbs. Zweiter Teil: Entwicklung der faunistischen Heimatsforschung. Dritter Teil: Die Weich- und Wirbeltiere des Bezirkes Scheibbs. Verlag R. \& F. Radinger. Scheibbs, 584 pp.

Retzlaff, H. \& Schulze, W., 1992. Mitteilungen zur Insektenfauna in Ostwestfalen. Lippe 6 (Saltatoria, Coleoptera, Raphidioptera, Trichoptera). Mitteilungen der Arbeitsgemeinschaft ostwestfälisch-lippischer Entomologen, 8: 27-31.

Reuter, O. M., 1894. Neuroptera Fennica. Förtecking och Beskrifning öfver Finlands Neuropterer. Acta Societatis pro Fauna et Flora Fennica, 9(8): 1-36.

Riek, E. F., 1967. Structures of unknown, possibly stridulatory, function on the wings and body of Neuroptera; with an appendix on other endopterygote orders. 
Australian Journal of Zoology, 15: 337-348. http://dx. doi.org/10.1071/ZO9670337

Rimski-Korsakow, M. N., 1922. K biologii werbludok (Rhaphidiidae). Trudy Tret'ego Vserossiiskogo entomofitopatologichekogo s"ezda, Petrograd, 1921. Guttenberg Typography. Petrograd: 36-43.

Rohdendorf, B. B., 1969. Paläontologie. Handbuch der Zoologie, 4(2): 1-27.

Röhricht, W., 1996. Netzflügler und Schnabelfliegen aus Mitteldeutschland. Naturwissenschaftliche Beiträge des Museums Dessau, 9: 135-156.

Röhricht, W. \& Tröger, E.J., 1998. Rote Liste der Netzflügler (Neuropteroidea). In: Bundesamt für Naturschutz: Rote Liste gefährdeter Tiere Deutschlands. Schriftenreihe für Landschaftsplege und Naturschutz, 55: 231-234.

Rohwer, S.A., 1909. Three New Fossil Insects from Florissant, Colorado. American Journal of Science, 28: 533536. http://dx.doi.org/10.2475/ajs.s4-28.168.533

Rösel von Rosenhof, A. J., 1755. Der monathlich-herausgegebenen Insecten-Belustigung Dritter Teil worinnen ausser verschiedenen, zu den in den beedeb erster Theilen enthalteten Classe, gehörigen Insecten, auch mancherley Arten von acht neuen Classen nach ihrem Ursprung, Verwandlung una anderen wunderbaren Eigenschafften, aus eigener Erfahrung beschrieben, und in sauber illuminirten Kupfern, nach del Leben abgebildet vorgestellt werden. J.J. Fleischmann. Nürnberg. $624 \mathrm{pp}$.

Rosenhauer, W. G., 1856. Die Thiere Andalusiens nach dem Resultate einer Reise zusammengestellt, nach den Beschreibungen von 249 neuen oder bis jetzt noch unbeschriebenen Gattungen und Arten. Theodore Blaesing. Erlangen. 429 pp.

Rostock, M., 1873. Neuropterologische Mittheilungen. Sitzungsberichte der Naturwissenschaftlichen Gesellschaft Isis in Dresden 1873: 9-17.

Rostock, M., 1881. Verzeichniss der Neuropteren Deutschlands (1), Oesterreichs (2) und Schweiz (3). Entomologische Nachrichten, 7: 217-228.

Rostock, M., 1888. Neuroptera germanica. Die Netzflügler Deutschlands mit Berücksichtigung auch einiger ausserdeutscher Arten nach der analytischen Methode unter Mitwirkung von H. Kolbe bearbeitet. Jahresbericht des Vereins für Naturkunde zu Zwickau, 1887: 1-198.

Rupprecht, R., 1975. Die Kommunikation von Sialis (Megaloptera) durch Vibrationssignale. Journal of Insect Physiology, 21: 305-320. http://dx.doi. org/10.1016/0022-1910(75)90026-8

Sankey, J. H. P., 1953. Fauna of the district around juniper hall., Mickleham, Surrey. The Entomologist's Monthly Magazine, 89: 284-285.

Saure, C., 1996. Die Kamelhalsfliegen (Raphidioptera) der Mark - Beitrag zur Tierwelt von Brandenburg und Berlin. Entomologische, Nachrichten und Berichte, 40, 1996/2: 75-82.

Saure, C. \& Gerstberger, M., 1991. Standardliste und Rote Liste der Neuropteroidea (Netzflügler s.l.) von Berlin. In: A. Auhagen, R. Platen \& H. Sukopp (eds.). Rote Listen der gefährdeten Pflanzen und Tiere in Berlin. Landschaftsentwicklung und Umweltforschung: 237-241.
Schimitschek, E., 1929. Teropium Gabrieli Weise und Teropium fuscum F. Ein Beitrag zu ihrer Lebensgeschichte und Lebensgemeinschaft. Zeitschrift für Angewandte Entomologie, 15: 229-334.

Schimitschek, E., 1931. Der achtzähnige Lärchenborkenkäfer Ips cembrae Heer. Zur Kenntnis seiner Biologie und Ökologie sowie seines Lebensvereines. Zeitschrift für Angewandte Entomologie, 17: 255-310.

Schirmer, C., 1912. Weitere Beiträge zur Kentnis der Insekten der Mark Brandenburg. Neuroptera genuina. Gruppe 2 Planipennia. Archiv für Naturgeschichte, 78A(7-12): 137-140.

Schlüter, T., 1986. The fossil Planipennia - a review. In: J. Gepp, H. Aspöck \& H. Hölzel (eds.). Recent Research in Neuropterology (Proceedings of the 2nd International Symposium on Neuropterology, Hamburg, 1984). Graz: 103-111.

Schmitz, O., 1992. Beitrag zur Netzflüglerfauna (Insecta: Neuropteroidea) von Köln und Umgebung. Decheniana, 31: 165-180.

Schmitz, O., 1993. Die Netzflügler (Neuroptera s.l.) des Naturschutzgebietes "Ahrschleife bei Altenahr" und angrenzender Weinbergsbrachflächen. Beiträge Landespflege Rheinland-Pfalz 16: 429-444.

Schneider, W. G., 1843. Monographia generis Rhaphidiae Linnaei. Continens et novas de huius generis singulis speciebus institutas observationes, et integram omnium, quae hucusque inventae sunt, specierum descriptionem. Grass, Barth \& Co. Vratislaviae. 96 pp.

Schneider, W. G., 1845. Vergleichung der von Rambur in seiner "Histoire naturelle des Insectes Neuroptères" pag. 436-440 beschriebenen Arten der Gattung Raphidia mit denen meiner "Monographia generis Raphidiae,” mit Beziehung auf Herrn Dr. Hagen's Bemerkungen im Jahrg. 1844 der entomologischen Zeitung No. 5. p. 180-185. Stettiner Entomologische Zeitung, 6: 250-256.

Schneider, W. G., 1885. Verzeichniss der Neuropteren Schlesiens. Zeitschrift für Entomologie (N.F.), 10: 17-32.

Schoch, G., 1885. Zusätze und Berichtigungen zur Fauna Neuropterorum helvetica. Fauna Insectorum Helvetiae, 1887: 89-94.

Schøyen, W. M., 1887. Fortegnelse over de i Norge hidtil observerede Neuroptera Planipennia og Pseudo-Neuroptera. Forhandlingar i Videnskabsselskabet i Kristiania, 13: 1-30.

SchummeL, T. E., 1832. Versuch einer genauen Beschreibung der in Schlesien einheimischen Arten der Gattung Raphidia, Linn. Beiträge zur entomologie besonders in bezug auf Schlesien, herausgegeben von T. E. Schummel. Eduard Pelz. Breslau. 16 pp.

Schwenke, W., 1958. Untersuchungen zum Massenwechsel der Kiefernspanner Bupalus piniarius L. und Semiothisa liturata Cl. auf vergleichend-biozönotischer Grundlage. Beiträge zur Entomologie, 4: 388-451.

Seifert, G., 1975. Entomologisches Prakticum. G. Thieme Verlag. Stuttgart. $430 \mathrm{pp}$.

Selys-Longchamps, E., 1888. Catalogue raisonné des Orthoptères et des Névroptères de Belgique. Annales de la Société Entomologique de Belgique, 32: 103-203. 
Séméria, Y., 1984. Raphidia (Xanthostigma) corsica Hagen (Insecta, Neuropteroidea) dans le Var, espèce nouvelle pour la faune de France. Nouv. Nouvelle Revue d'Entomologie (N.S.), 1: 178.

Séméria, Y. \& Berland, L., 1988. Atlas des Névroptères de France et d'Europe. Mégaloptères, Raphidioptères, Névroptères Planipennes, Mécoptères. Nouvelle [2nd] édition revue et augmentée. Boubée. Paris. 190 pp.

Ševčik, J., 1995. Dlouhošíky (Insecta: Neuropteroidea: Raphidioptera) Slezska a severní Moravy - současný stav znalostí. Časopis Slezského Musea v Opave, 44: 251-258.

Sharp, D., 1895. Insects Part 1. Introduction, Aptera, Orthoptera, Neuroptera, and a portion of Hymenoptera (Sessiliventres and Parasitica). In: S. F. Harmer \& A. E. Shipley. The Cambridge Natural History, 5. MacMillan \& Co. London: 83-565.

Shaw, E., 1903. Orthoptera and Neuroptera in Lincolnshire and Nottinghamshire. The Entomologist's Monthly Magazine, 39: 91-95.

Siebold, C. Th. v., 1848. Lehrbuch der vergleichenden Anatomie der Wirbellosen Thiere. Veit \& Comp. Berlin. 679 pp.

Silvestri, F., 1943. Ordo Neuroptera Ordo Neuroptera. In: Compendio di Entomologia Applicata (Agraria - Forestale - Medica - Veterinaria), Vol. 2. Ernesto Della Torre. Portici: 1-33.

Snodgrass, R. E., 1957. A revisioned interpretation of the external preproductive organs of male insects. Smithsonian Miscellaneous Collections, 135(6): 1-60.

Starý, B., Bezděčka, P., Čapek, M., Starý, P., Zelený, J. \& Šedivý, J., 1988. Atlas of insects beneficial to forest trees, Vol. 2. Elsevier Science. Amsterdam. 100 pp.

Starý, B., Bezděčka, P., Čapek, M., Starý, P., Zelený, J. \& Šedivý, J., 1990. Atlas nützlicher Forstinsekten. Deutscher Landwirtschaftsverlag. Berlin. 104 pp.

Stein, F., 1837. Bemerkungen zu den von Herrn Schummel augestellten Arten der Gattung Raphidia L. Isis, 1837: 98-102.

Stein, F., 1838. Entwicklungs-Geschichte mehrere Insektengattungen aus der Ordnung der Neuropteren. Archiv für Naturgeschichte, 4: 315-333.

Steinmann, H., 1963. Raphidiopterological Studies I. Navasana gen. n. from Hungary, Harraphidia gen.n. from Morocco, New Lesna NAV. and Subilla NAV. species from Europe. Acta Zoologica Academiae Scientiarum Hungaricae, 9: 183-198.

Steinmann, H., 1964. Raphidiopterological studies 2. New Raphidia L. and Rhaphidilla Nav. species from Europe and Asia. Acta Zoologica Hungarica, 10: 199-227.

Steinmann, H., 1967. Tevenyakú fátyolkák, Vízifátyolkák, Recésszárnyúak és Csőrös rovarok. Raphidioptera, Megaloptera, Neuroptera és Mecoptera. Acta Zoologica Hungarica, 82: 1-203.

Steinmann, H. \& Zombori, L., 1984. A morphological atlas of insects larvae. Akadémai Kiadó. Budapest. 403 pp.

Steinmann, H. \& Zombori, L., 1985. An atlas of insect morphology, $2^{\text {nd }}$ revised edition. Akadémai Kiadó. Budapest. 253 pp.
Stelzl, M., 1989. Nahrungsspektren mitteleuropäischer Neuropteren-Imagines (Neuropteroidea, Insecta), mit einer Diskussion über deren Nützlichkeit als Opponenten von Pflanzenschädlingen. Diplomarbeit Universität Graz. 145 pp.

Stelzl, M., 1991. Untersuchungen zu Nahrungsspektren mittelauropäischer Neuropteren - Imagines (Neuropteroidea, Insecta). Mit einer Diskussion über deren Nützllichkeit als Opponenten von Pflanzenschädlingen. Zeitschrift für Angewandte Entomologie, 111: 469-477.

Stephens, J. F., 1829. A systematic catalogue of British insects: being an attempt to arrange all the hitherto discovered indigenous insects in accordance with their natural affinities containing also the references to every English writer on entomology, and to the principal foreign authors with all the published British genera to the present time. Baldwin \& Cradock. London. 388 pp.

Stephens, J.F., 1836. Illustrations of British entomology; or a synopsis of indigenous insects: contaiming their generic and specific distinctions; with am account of their metamorphoses, times of appearance, localities, food, and economy, as far as practicable. Vol. 6. Mandibulata. Baldwin \& Cradock. London. 240 pp.

Stitz, H., 1909. Zur Kenntnis des Genitalapparats der Neuropteren. Zoologische Jahrbücher, 27: 377-448.

Stitz, H., 1927a. Ordnung: Netzflügler, Neuroptera. Die Tierwelt Mitteleuropas, 6(1): 1-24.

Stitz, H., 1927b. Raphidiina. Biologie der Tiere Deutschlands, 35: 49-66.

Strobl, G., 1906. Neuropteroiden (Netzflügler) Steiermarks (und Niederöstereichs). Mitteilungen des Naturwissenschaftlichen Vereins für Steiermark, 42: 225-266.

Sulzer, J. H., 1761. Die Kennzeichen der Insekten, nach Anleitung des Königl. Schwed. Ritters und Leibarztes Karl Linnaeus, durch 24: Kupfertafeln erläutert und mit der selben natürlichen Geschichte begleitet. Heidegger \& Co. Zürich. 68 pp.

Suntrup, A., 1990. Untersuchungen zur Faunistik und Autökologie von Netzflüglern (Insecta: Neuropteroidea) in Norddeutschland, 2 vols. Diplomarbeit. Zoologisches Institut, Georg-August-Universität. Göttingen. 65 pp.+76 pp.

Syms, E. E., 1934. Biological Notes on British Megaloptera. Proceedings of the South London Entomological and Natural History Society, 1934-1935: 121-124.

Szilady, Z., 1912. II. Neuroptera et Pseudoneuroptera. Rovartani Lapok, 19: 53-58.

Sziráki, G., 1996. Ecological investigations of the Neuropteroidea of oak forests in Hungary (Insecta: Raphidioptera, Neuroptera). In: M. Canard, H. Aspöck, H. \& M. W. Mansell (eds). Pure and Applied Research in Neuropterology (Proceedings of the Fifth International Symposium on Neuropterology, Cairo, 1994). Toulouse: 229-232.

Sziráki, G., 1999. Neuropteroidea of the Aggtelek National Park. In: S. Mahunka (ed.). Fauna of the Aggtelek National Park, Volume I. Hungarian Natural History Museum. Budapest: 143-149.

Sziráki, G., Ábrahám, L., Szentkirályi, F. \& Papp, Z., 1992. A check-list of the Hungarian Neuropteroidea 
(Megaloptera, Raphidioptera, Planipennia). Rovartani Kölzlemények, 52: 113-118.

Sziráki, G. \& Popov, A., 1996. Neuropteroidea of the Bükk National Park. In: S. Mahunka (ed.). The Fauna of the Bükk National Park, Volume 2. Hungarian Natural History Museum. Budapest: 389-396.

Tauber, C. A., 1987. Order Raphidioptera. In: F.W. Stehr (ed.). Immature Insects. Kendall/Hunt Publishing. Dubuque: 123-125.

Thenius, E., 2000. Lebende Fossilien. Oldtimer der Tierund Pflanzenwelt - Zeugen der Vorzeit. Verlag Friedrich Pfeil. München. 228 pp.

Thompson, W. R., 1950. A catalogue of the parasites and predators of insect pests. Section I. Parasite host catalogue. Part II. Parasites of the Neuroptera, Odonata, Orthoptera, Psocoptera, Siphonaptera and Thysanoptera. Commonwealth Institute of Biological Control. Ottawa. iv+35 pp.

Tillyard, R. J., 1919. The Panorpoid Complex. Part 3: The Wing-Venation. Proceedings of the Linnean Society of New South Wales, 44(3): 533-718.

Tillyard, R. J., 1932. Kansas Permian insects. Part 14. The Order Neuroptera. American Journal of Science, 23: 1-30. http://dx.doi.org/10.2475/ajs.s5-23.133.1

Titschack, E., 1930. Die Copeognatha, Megaloptera, Neuroptera und Mecoptera der näheren und weiteren Umgebung Hamburgs. Verhandlungen des Vereins für Naturwissenschaftliche Heimatsforschung, 21: 104-107.

Tjeder, B., 1928. Preliminary notes concerning Hemerobius fuscescens Wallengr. and Raphidia laticeps Wallengr. Entomologisk Tidskrift 49: 162.

Tjeder, B., 1937a. A contribution to the phylogeny of Dilaridae and the Raphididae. (Neuroptera). Opuscula Entomologica, 3: 138-148.

Tjeder, B., 1937b. Geographical and synonymical notes on some Raphidiidae and Sialidae. 1. Some notes on Inocellia crassicornis. 2. The Swedish Raphidia-species. Opuscula Entomologica, 3: 118-124.

Tjeder, B., 1938. Mecoptera, Neuroptera, and Trichoptera from the southern part of the Swedish East Coast. Göteborgs Kungliga Vetenskaps- och Vitterhets-samhalles Handlingar, Serie B, 6(3): 1-34.

Tjeder, B., 1940a. Revisions of Scandinavian Neuroptera (s.str.) and Mecoptera recorded by J.W. Zetterstedt, H.D.J. Wallengren, and others. Opuscula Entomologica, 5: 67-114.

Tjeder, B., 1940b. Catalogus Insectorum Sueciae. 1. Neuroptera et Mecoptera. Opuscula Entomologica, 5: 117-121.

Tjeder, B., 1941. Plecoptera, Mecoptera, Neuroptera, and Trichoptera collected by Mr. Hans Lohmander in the Island of Bornholm in the Baltic. Göteborgs Kungliga Vetenskaps- och Vitterhets-samhalles Handlingar, 1: 1-25.

Tjeder, B., 1943a. Revision of the Norwegian Neuroptera and Mecoptera recorded by W.M. Schoyen. Norsk Entomologisk Tidsskrift, 6: 133-139.

Tjeder, B., 1943b. The Neuroptera and Mecoptera of Northern Norway (Nordland, Troms and Finnmark). Tromso Museums Årshefter, 63(3): 1-15.
Tjeder, B., 1954. Genital structures and terminology in the order Neuroptera. Entomologiske Meddelelser, 27: 23-40.

Tjeder, B., 1964. Recension. Opuscula Entomologica, 29: 108-109.

Tjeder, B., 1970. Neuroptera. In: S. L. Tuxen (ed.). Taxonomist's glossary of genitalia in insects. Munksgaad. Copenhagen: 89-99.

Tjeder, B., 1972. Reviderad Förteckning över sveriges Neuroptera och Mecoptera. Entomologen, 1: 21-26.

Tröger, E. J., 1993. Beitrag zur Kenntnis der Netzflügler in Franken. Nachrichtenblatt der Bayerischen Entomologen, 42(2): 32-46.

Tröger, E. J., 1997. Netzflügler (Neuropteroidea) in Vogelnesten. Mitteilungen des Badischen Landesvereins für Naturkunde und Naturschutz e.V. Freiburg im Breisgau, 16(3/4): 581-586.

Tröger, E. J., 2002. Netzflügler (Neuropterida) in Franken. Galathea, 13 Supplement: 37-70.

Tuxen, S. L. (Editor). 1970. Taxonomist's Glossary of Genitalia in Insects. $2^{\text {nd }}$ Edition. Munksgaard. Copenhagen. 359 pp.

Ujhelyi, S., 1979. Data of some orders of Insecta ocurring in Bakony mountain. Veszprém megyei múzeum közleményei, 14: 85-93.

Van der Ploeg, D.T.E., 1985. In jurist as amateur-biolooch mr. Herman Albarda 1826-1898. Fryske Akademy. Ljouwest/ Leeuwarden. 87 pp.

Vannier, G. \& Condé, B., 1962. Entomologie Générale: Notes biologiques sur quelques Raphidioptères de la faune francaise. Bulletin de la Société Entomologique de France, 67: 96-104.

Vas, J., Abraham, L. \& Markó, V., 2001. Methodological Investigations on a Neuropteroidea Community. Acta Phytopathologica et Entomologica Hungarica, 36(1-2): 101-113.

Verdcourt, B., 1977. A visit to Aspley Heath Bedfordshire Naturalist, 28(29): 1.

Verdcourt, B., 1979. Lacewing flies (Neuroptera). Report of the recorder. Bedfordshire Naturalist, 33: 58-60.

Verdcourt, B., 1980. Lacewing flies (Neuroptera). Report of the Recorder. Bedfordshire Naturalist, 34: 40.

Wachmann, E. \& Saure, C., 1997. Netzflügler, Schlammund Kamelhalsfliegen: Beobachtung - Lebensweise. Naturbuch Verlag. Augsburg. 159 pp.

Wakeman, M., 1960. Raphidia maculicollis Steph. (Megaloptera, Raphidiidae) in Wyre Forest, Worcester. The Entomologist's Monthly Magazine, 96: 128.

Walker, F., 1853. List of the specimens of neuropterous insects in the collection of the British Museum. Part II. (Sialides-Nemopterides). British Museum. London. [iii]+pp. 193-476.

Wallengren, H. D. J., 1863. Bidrag till kännedomen af Sveriges Neuroptera. Öfversigt af Kongliga Vetenskaps-Akademiens Forhändlingar, 20: 15-26.

Wallengren, H. D. J., 1871. Skandinaviens Neuroptera. Första Avdelningen Neuroptera, Planipennia. Kungliga Svenska Vetenskapsakademiens Handlingar, 9: 1-76. 
Wanach, B., 1915. Die Neuropterenfauna Potsdams. Deutsche Entomologische Zeitschrift, 1915: 323-326.

Wang, W., 1987. Early Mesozoic insects fossils from western Liaoning. In: X.-h. Yu, W.-l. Wang, X.-t. Liu \& W. Zhang (eds.). Mesozoic stratigraphy and palaeontology of western Liaoning, Volume 3. Geological Publishing House. Beijing: 202-222.

Waterhouse, G. R., 1836. V. Description of the Larva and Pupa of Raphidia ophiopsis. Transactions of the [Royal] Entomological Society of London, 1: 23-27. http://dx. doi.org/10.1111/j.1365-2311.1837.tb03172.x

Weber, H., 1939. Beobachtungen über die Wirkungen von Empusa weberi Lakon auf die Larve von Raphidia ophiopsis L. Zeitschrift für Angewandte Entomologie, 26: 522-535.

Weele, H. W. van der, 1907. Eerste Supplement op den Catalogus der Nederlandsche Neuropteroidea. Tijdschrift voor Entomologie, 40: 121-128.

Weele, H. W. van der, 1910. Megaloptera (Latreille) Monographic Revision. Collections Zoologiques du Baron E. de Selys Longchamps. Catalogue Systématique et Descriptif, 5: 1-93.

Weidner, H., 1982. Morphologie, Anatomie und Histologie. Handbuch der Zoologie, 4/2(1/11): 1-531.

Weiss, V., 1947. 259. (Raphid.) Erster Beitrag zur Kenntniss der Raphidiina. Bombus, 33: 146.

Westwood, J. O., 1840. An introduction to the modern classification of insects; founded on the natural habits and corresponding organisation of the different families. Longham \& Co. London. 587 pp.

Whiting, M. F., Carpenter, J. C., Wheeler, Q. U. \& Wheeler, W. C., 1997. The Strepsiptera problem: Phylogeny of the holometabolous insect orders inferred rom 18S and 28S ribosomal DNA sequences and morphology. Systematic Biology, 46: 1-68. http://dx.doi.org/10.1093/sysbio/46.1.1

Wichmann, H. E., 1957. Untersuchungen an Ips typographus L. und seiner Unterwelt. Zeitschrift für Angewandte Entomologie, 40: 433-440.

Williams, C. B., 1913. Some biological notes on Raphidia maculicollis, Steph. The Entomologist, 46: 6-8.

Willmann, R., 1994. Raphidiodea aus dem Lias und die Phylogenie der Kamelhalsfliegen (Insecta: Holometabola). Paläontologische Zeitschrift, 68(1/2): 167-197. http://dx.doi.org/10.1007/BF02989439

Wilson, F., 1938. Notes on the insect of chermes with particular reference to Pineus pini, Koch, and P. strobi, Hartig. Bulletin of Entomological Research, 29: 373-389. http://dx.doi.org/10.1017/S0007485300026286

Winterton, S. L., Hardy, N. B. \& Wiegmann, B. M., 2010. On wings of lace: phylogeny and Bayesian divergence time estimates of Neuropterida (Insecta) based on morphological and molecular data. Systematic Entomology, 35: 349-378. http://dx.doi.org/ 10.1111/j.1365-3113.2010.00521.x

Withycombe, C. L., 1923. The wing venation of Raphidia maculicollis Stephens. The Entomologist, 56: 33-35.

Woglum, R. S. \& McGregor, E. A., 1958. Observations on the life history and morphology of Agulla bractea Carpenter (Neuroptera: Raphidiodea: Raphidiidae). Annals of the Entomological Society of America, 51: 129-141. http://dx.doi.org/10.1093/aesa/51.2.129

Woglum, R. S. \& McGregor, E. A., 1959. Observations on the life history and morphology of Agulla astuta (Banks), (Neuroptera: Raphidiodea: Raphidiidae). Annals of the Entomological Society of America, 52: 489-502. http://dx.doi.org/10.1093/aesa/52.5.489

Wüstnei, W., 1902. Beiträge zur Insektenfauna SchleswigHolsteins. 7. Verzeichnis der von mir in SchleswigHolstein beobachteten Neuroptera Planipennia. Schriften des Naturwissenschaftlichen Vereins für SchleswigHolstein, 12: 128-135.

Wyniger, R., 1974. Insektenzucht. Methoden der Zucht und Haltung von Insekten und Milben im Laboratorium. E. Ulmer. Stuttgart. 368 pp.

Yang, Ch. K., 1978. Atlas of natural enemies of economic insects of China. Academia Sinica, Institute of Zoology, \& Science Press. Beijing. iii+300 pp.

Zabel, J., 1941. Die Kamelhalsfliege. Beiträge zur Biologie der Raphidia ophiopsis. Natur und Volk, 71: 187-196.

Zacwilichowski, J., 1938. Materialien zur NeuropterenFauna Polens. Fragmenta Faunistica Musei Zoologici Polonici, 3(15): 242-249.

Zacwilichowski, J., 1939. Dalsze materialy do fauny sieciarek (Neuroptera) Polski. Weitere Materialien zur Neuropteren-Fauna Polens. Fragmenta Faunistica Musei Zoologici Polonici, 4: 1-6.

Zelený, J., 1969. Variability in the species Raphidia ophiopsis Linnaeus, and Agulla xanthostigma (Schummel) (Raphidioptera). Acta Entomologica Bohemoslovaca, 66: 15-38.

Zelený, J., 1971. Megaloptera, Raphidioptera, Neuroptera und Mecoptera - Arten des Gebirges Novohradské hory. Sborník Jihoceského Muzea v Ceskych Budejovicích Prírodní Vedy, 11: 39-43.

Zelený, J., 1977. Megaloptera, Raphidioptera, Planipennia (Neuroptera). Enumeratio Insectorum Bohemoslovakie. Sborník faunistickych prací Entomologického oddelení N rodního Musea v Praze, 15(Supp 4): 125-131.

Zetterstedt, J. W., 1840. Insecta Lapponica. Ordo 7. Neuroptera. L. Voss. Lipsiae. vi+1140 pp. 